\title{
Fate, survival and growth of fecal coliforms through centrifuge dewatering
}

\author{
By \\ Julie Gardner, B.Sc. Env. Science
}

A thesis submitted to the Faculty of Graduate Studies and Research

in partial fulfillment of the requirements for the degree of

Master of Applied Science

in

\section{Environmental Engineering*}

Department of Civil and Environmental Engineering Carleton University

Ottawa, Ontario

Canada

\footnotetext{
*The Master of Applied Science in Environmental Engineering Program is a joint program with the University of Ottawa administered by the Ottawa-Carleton Institute for Environmental Engineering
}

(C) Copyright, 2007 


$\begin{array}{ll}\begin{array}{l}\text { Library and } \\ \text { Archives Canada }\end{array} & \begin{array}{l}\text { Bibliothèque et } \\ \text { Archives Canada }\end{array} \\ \begin{array}{l}\text { Published Heritage } \\ \text { Branch }\end{array} & \begin{array}{l}\text { Direction du } \\ \text { Patrimoine de l'édition }\end{array} \\ \begin{array}{l}\text { 395 Wellington Street } \\ \text { Ottawa ON K1A 0N4 } \\ \text { Canada }\end{array} & \begin{array}{l}\text { 395, rue Wellington } \\ \text { Ottawa ON K1A ON4 } \\ \text { Canada }\end{array}\end{array}$

Your file Votre référence ISBN: 978-0-494-33647-2 Our file Notre référence ISBN: 978-0-494-33647-2

NOTICE:

The author has granted a nonexclusive license allowing Library and Archives Canada to reproduce, publish, archive, preserve, conserve, communicate to the public by telecommunication or on the Internet, loan, distribute and sell theses worldwide, for commercial or noncommercial purposes, in microform, paper, electronic and/or any other formats.

The author retains copyright ownership and moral rights in this thesis. Neither the thesis nor substantial extracts from it may be printed or otherwise reproduced without the author's permission.
AVIS:

L'auteur a accordé une licence non exclusive permettant à la Bibliothèque et Archives Canada de reproduire, publier, archiver, sauvegarder, conserver, transmettre au public par télécommunication ou par l'Internet, prêter, distribuer et vendre des thèses partout dans le monde, à des fins commerciales ou autres, sur support microforme, papier, électronique et/ou autres formats.

L'auteur conserve la propriété du droit d'auteur et des droits moraux qui protège cette thèse. $\mathrm{Ni}$ la thèse ni des extraits substantiels de celle-ci ne doivent être imprimés ou autrement reproduits sans son autorisation.
In compliance with the Canadian

Privacy Act some supporting forms may have been removed from this thesis.

While these forms may be included in the document page count, their removal does not represent any loss of content from the thesis.
Conformément à la loi canadienne sur la protection de la vie privée, quelques formulaires secondaires ont été enlevés de cette thèse.

Bien que ces formulaires aient inclus dans la pagination, il n'y aura aucun contenu manquant.

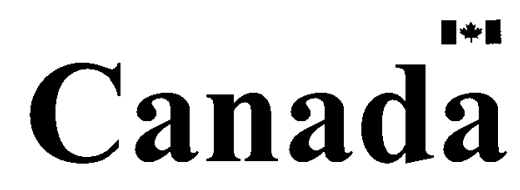




\begin{abstract}
Recent studies have reported significant increases in the counts of indicator bacteria for anaerobically digested sludge samples following centrifugal dewatering. As bacterial enumeration at the treatment plant is done immediately following digestion, this could cause samples to exceed regulatory standards for land disposal. The study was divided into two phases. In the first phase it was found that the centrifuge shear, as well as the polymer type and dose did not significantly affect the fecal coliform counts through a laboratory centrifuge. In the second phase, although immediate reactivation of fecal coliforms was not observed, significant regrowth was detected for cake samples at one of two treatment plants. This regrowth was only possible for samples in the absence of centrate. Chemical characterisation showed that the full-scale centrate had significantly higher sulphide levels and provided a more stressful environment for the fecal coliforms than centrate from a laboratory centrifuge. Counts of total bacteria in the samples were not consistent with the counts of the culturable fecal coliforms.
\end{abstract}




\section{Acknowledgements}

Firstly, I would like to extend sincere thanks to my supervisor, Dr. Banu Örmeci, for her guidance, her excellent supervision during the entirety of the thesis, her timely and comprehensive comments as well as her many trips to the treatment plants. To the members of the committee, I thank you for your comments and your review of this work. Also, to the laboratory staff in the Environmental Engineering laboratory at Carleton University, especially to Dr. Marie-Josée Tudoret, for all her help, her ability to get things done and keep the laboratory safe and clean for all of us. I also want to thank the other students working in the lab for their help and advice, especially Ms. Audrey Murray for mixing up some fresh polymers for me whenever I needed them and helping me with the Floccky tester for the optimum polymer dose calculations. To the staff of the treatment plants for always being so accommodating and welcoming. To the Ontario Graduate Scholarships for helping with my funding and allowing me to focus on my research. Finally, I want to thank my family and those most dear to me, for their constant support and their patience throughout my work. 


\section{Table of Contents}

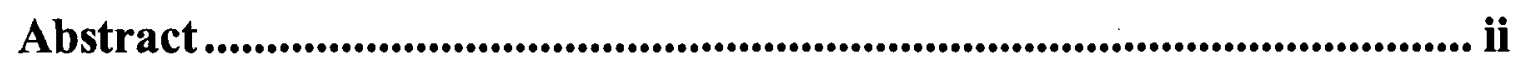

Acknowledgements .................................................................................................... iii

Table of Contents ................................................................................................... iv

List of Tables............................................................................................. vii

List of Figures .................................................................................... viii

List of Acronyms and Nomenclature ..........................................................x

1. Sludge Treatment Overview.................................................................1

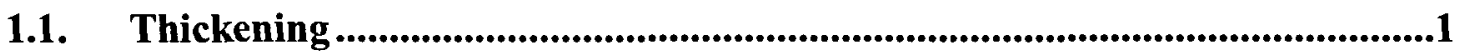

1.2. Stabilisation .................................................................................................2

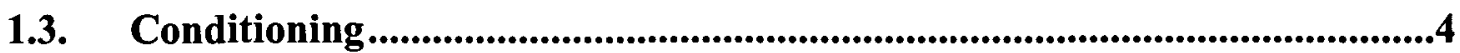

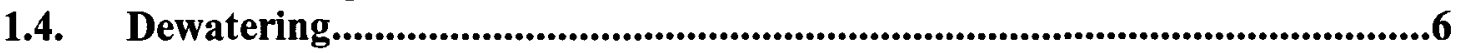

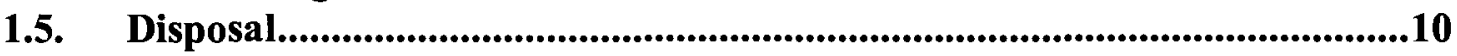

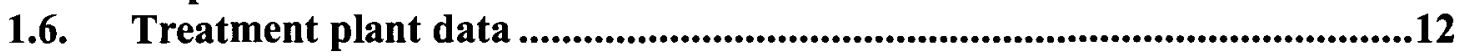

2. Literature Review ..............................................................................16

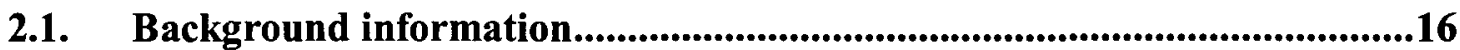

2.1.1. Regrowth / Reactivation ................................................................16

2.1.2. Viable but Non Culturable Bacteria ...................................................18

2.1.3. Enumeration methods ......................................................................20

2.2. Conditions of observed regrowth / reactivation ......................................22

2.2.1. Centrifuge versus belt press dewatering................................................22

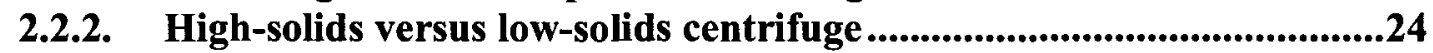

2.2.3. Change in temperature post digestion ......................................................24

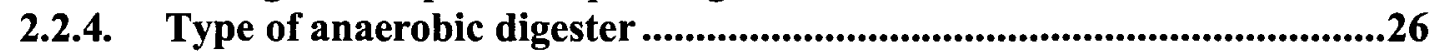

2.3. Topics to be examined further from current study......................................27

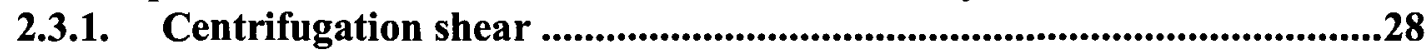

2.3.2 Conditioning with polymers ...................................................................29 
2.3.3 Viable versus culturable counts ...................................................................31

2.3.4 Centrate characterisation............................................................................32

2.4 Objectives ................................................................................................................34

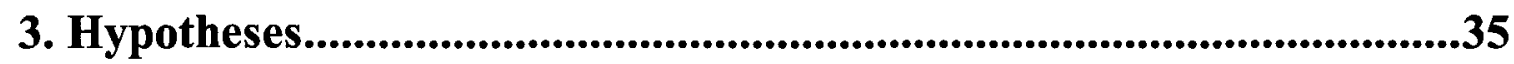

4. Materials and Methods ...................................................................37

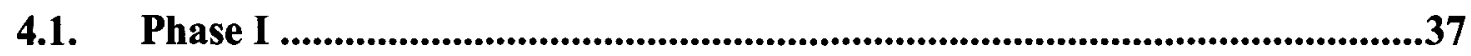

4.1.1. Experimental design ...................................................................................37

4.1.2. Treatment plant characteristics.................................................................41

4.1.3. Anaerobically digested samples ......................................................................41

4.1.4. Polymers used .......................................................................................................42

4.1.5. Determination of the optimum polymer dose ..............................................43

4.1.6. Bacterial enumeration ........................................................................45

4.1.7. Statistical analysis...........................................................................................46

4.2. Phase II .................................................................................................................46

4.2.1. Experimental design ......................................................................................47

4.2.2. Treatment plant sampling.............................................................................52

4.2.3. Lab-scale centrate sampling.................................................................53

4.2.4. Specific methodology for centrate parameters ............................................54

4.2.5. Sterilisation of centrate .....................................................................65

4.2.6. Methodology for fecal coliform seeding.................................................66

4.2.7. Cellular ATP methodology.............................................................................67

4.2.8. Bacterial enumeration ....................................................................................69

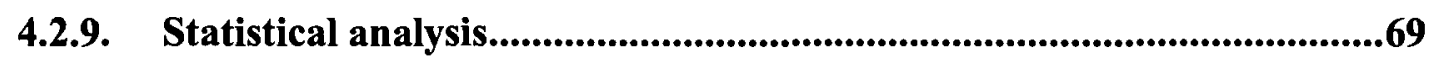

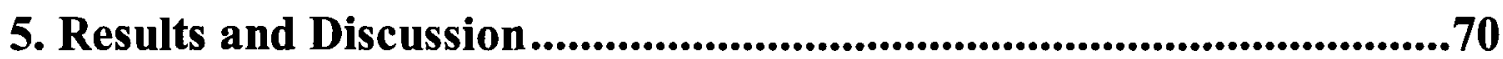

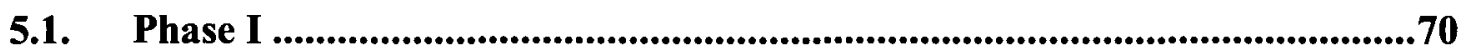

5.1.1. Test 1: Effect of centrifuge speed ..................................................................70

5.1.2. Test 2: Addition of polymer dose and type.....................................................78

5.1.3 Simulation of full-scale centrifuges in the lab ............................................86

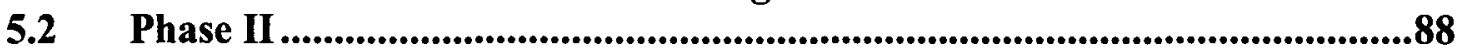

5.2.1 Test 1: Full-scale samples......................................................................................88

5.2.2 Test 2: Cake and centrate sample aging at different temperatures......94

5.2.3 Test 3: Centrate characterisation .............................................................109

5.2.4 Test 4: Centrate seeding.........................................................................113

5.2.5 Test 5: Cellular ATP .....................................................................119 


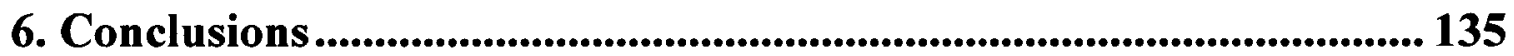

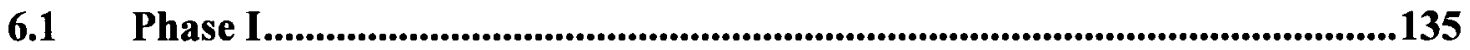

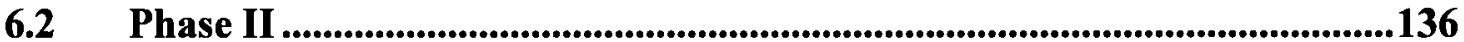

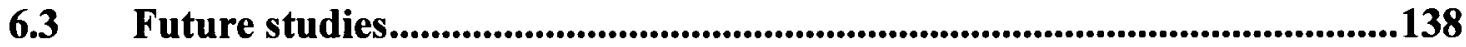

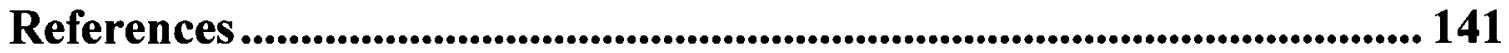

Appendix A: Raw Data................................................................................. 158

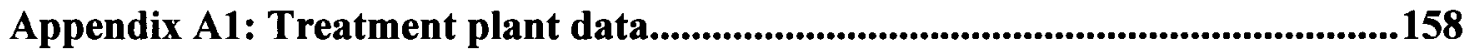

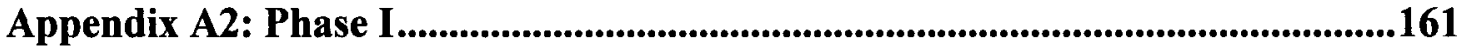

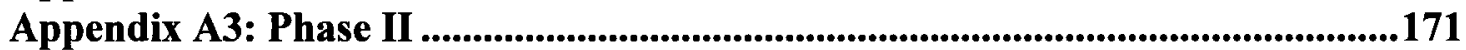




\section{List of Tables}

Table 1.1: Pathogen reduction requirements $\quad$................................................................11

Table 1.2: Treatment train characteristics ...........................................................13

Table 4.1: Treatment plant flow, polymer type and dose ............................................41

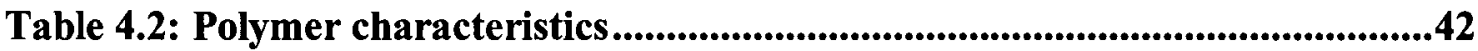

Table 5.1: Average cake and centrate solid concentrations (Centra GP8)...............77

Table 5.2: Centrate Characterisation ...........................................................................111

Table 5.3: $t$-test for the comparison of centrate characterisation parameters......112 


\section{List of Figures}

Figure 1.1: Countercurrent solid-bowl centrifuge .................................................8

Figure 1.2: Belt filter press .............................................................................9

Figure 1.3: $E$ coli concentrations in digested sludge and dewatered cake at

Treatment Plant A............................................................................14

Figure 4.1: Treatment train locations of full-scale samples .....................................48

Figure 4.2: Samples types for Test 2 of Phase II .......................................................49

Figure 4.3: Colour response curve for BSA $(0-350 \mathrm{mg} / \mathrm{l})$...................................61

Figure 4.4: Colour response curve for galactose $(0-200 \mathrm{mg} / \mathrm{L})$...........................63

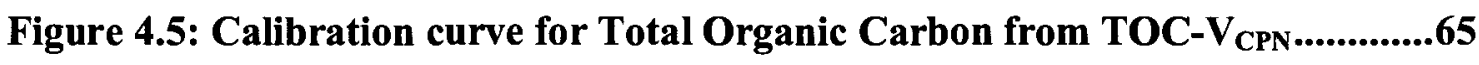

Figure 5.1: Effect of centrifuge speed with LTB/EC media .................................72

Figure 5.2: Effect of centrifuge speed with A-1 media ..............................................74

Figure 5.3: Effect of centrifuge speed with A-1 media and CA4600 polymer ..........76

Figure 5.4: Effect of polymer (CA4600) and centrifugation (4500 rpm) .................79

Figure 5.5: Effect of polymer (CA4600) and centrifugation (10,000rpm) ..............82

Figure 5.6: Effect of polymer (SD2061) and centrifugation (10,000rpm).................84

Figure 5.7: Full-scale sampling, Treatment Plant A ................................................90

Figure 5.8: Full-scale sampling, Treatment Plant B............................................91

Figure 5.9: Preliminary testing: Cake aging, Treatment Plant A..........................92

Figure 5.10: Preliminary testing: Cake and pellet aging Treatment Plant B..........93

Figure 5.11: Full-scale, Treatment Plant A sample aging ....................................96

Figure 5.12: Lab-scale, Treatment Plant A sample aging ....................................99

Figure 5.13: Full-scale, Treatment Plant B sample aging ...................................101

Figure 5.14: Lab-scale, Treatment Plant B sample aging ...................................104

Figure 5.15: Full-scale, Treatment Plant A sample aging, Run \#2 ........................108

Figure 5.16: $E$ coli seeding......................................................................117

Figure 5.17: BSI (\%) for Full-scale Treatment Plant A......................................121

Figure 5.18: BSI (\%) for centrate samples, Treatment Plant A ..........................122

Figure 5.19: BSI (\%) for centrate samples with aging, Treatment Plant A ..........124 
Figure 5.20: Full-scale ATP concentrations and fecal coliform values Treatment Plant A.................................................................................126

Figure 5.21: Centrate ATP concentrations and fecal coliform values Treatment Plant A.

Figure 5.22: Cake ATP concentrations and fecal coliform values with aging Treatment Plant A................................................................................128

Figure 5.23: Lab-scale centrate ATP and fecal coliform values with aging Treatment Plant A......................................................................................130

Figure 5.24: Full-scale centrate ATP and fecal coliform values with aging Treatment Plant A.....................................................................................132 


\section{List of Acronyms and Nomenclature}

A-1 A-1 Medium for the detection of fecal coliforms

ABR Active Biomass Ratio

ANOVA Analysis of Variance

ATP Adenosine Triphosphate

BSA Bovine Serum Albumin

cfu Coliform Forming Units

COD Chemical Oxygen Demand

CST Capillary Suction Time

DNA Deoxyribonucleic acid

E coli Escherichia coli

EQ Exceptional Quality biosolids

g DW grams Dry Weight

HPC Heterotrophic Plate Counts

LSD Least Significant Difference statistic

LTB / EC Lauryl Tryptose Broth / EC medium for the detection of fecal

Coliforms

MLSS Mixed Liquor Suspended Solids

MPN Most Probable Number

MTF Multiple Tube Fermentation

PBS Phosphate-Buffered Solution 
PCR Polymerase Chain Reaction

CPCR competitive PCR

rt-PCR reverse transcriptase PCR

R2A non-selective, nutrient poor agar

RPM Revolutions per Minute

TOC Total Organic Carbon

TPAD Temperature-Phased Anaerobic Digester

US EPA United States Environmental Protection Agency

UV Ultra Violet

VBNC Viable but Non Culturable

WAS Waste Activated Sludge

WERF Water Environment Research Foundation

$\mathrm{g}$ acceleration times gravity 


\section{Chapter 1}

\section{Sludge Treatment Overview}

Sludge treatment and disposal for municipal wastewater treatment plants can be undertaken in a variety of different ways depending on the characteristics of the sludge and the municipality. The numerous ways to treat municipal sewage will not be examined in detail in this chapter and only the processes related to the topic will be considered. A typical treatment train includes the thickening of incoming sludge, either Waste Activated Sludge (WAS) and/or raw sludge, followed by the stabilisation of the sludge, most often done by anaerobic digestion. Through stabilisation, the pathogen levels are lowered to an acceptable standard. Following this, the goal of the treatment processes is to reduce the quantity of sludge or biosolids that must be disposed of. Typically, conditioning of the sludge is undertaken prior to dewatering in order to improve the efficiency of the processes. The final disposal method depends on the sludge characteristics, such as its solid concentration, its level of disinfection and the concentration of nutrients and heavy metals. Each of these processes will be briefly discussed within this chapter. Reference will be made to the two treatment plants studied in this research which will be presented in more detail at the end of this chapter.

\subsection{Thickening}

The goal of the thickening of sludge is to diminish the volume of the sludge that must be treated. Because of this volume reduction, the treatment processes that follow can be designed to handle a smaller volume which saves money and delays infrastructure refurbishment. Thickened sludge typically has a maximum solids concentration of $7 \%$ 
which means it can still be pumped even though a large volume of water has been removed, making it easier to work with. Gravity thickening is a common means for the thickening of raw sludge because it settles easily. Waste activated sludge, however, has lighter particles which are harder to thicken and for this reason, a mechanical thickening device, such as a centrifuge, can be undertaken (Metcalf \& Eddy Inc., 2003). Thickener centrifuges operate at relative centrifugal forces of 500 to 3000 times that of gravity (Task Force on Sludge Thickening, 1980). Treatment Plant A uses centrifuge thickening for their WAS resulting in a thickened WAS with an average solids concentration of $6 \%$.

\subsection{Stabilisation}

Raw sludge and WAS can have very high pathogen counts and it is the goal of stabilisation to lower the counts of pathogens and indicator organisms as well as eliminating odours, making it easier to handle. There are many ways of stabilising sludge, including: anaerobic digestion, aerobic digestion, lime treatment, composting, chlorination, pasteurisation, irradiation, drying and incineration. The most common stabilisation process is anaerobic digestion; it provides stable sludge through the fermentation of the organic material by anaerobic bacteria. Anaerobic digestion has the added benefit of producing usable methane (Task Force on Wastewater Residuals Stabilization, 1995).

Within the digester, the process microbiology is often split into a three-step scheme; the first step being hydrolysis and fermentation, the second being hydrogen and acetic acid formation and the third being methane formation (Parkin and Owen, 1986). The final 
step in the process of anaerobic digestion is the most important one because methane is produced which can be used within the treatment plant. Methanogenic bacteria have slow growth rates and this step is thus also the rate-limiting step (Metcalf \& Eddy Inc., 2003).

Environmental factors such as $\mathrm{pH}$, alkalinity and temperature should be regulated and toxic substances need to be monitored within the digester. Many substances have been identified as inhibitors to microbial growth especially to methanogenic bacteria. Lipids, or long-chain fatty acids, have been shown as inhibitory to anaerobic digestion for many different types of biological sludges (Hwu et al., 1996; Lalman and Bagley, 2001; Rinzema et al., 1994). Protein degradation can release levels of ammonia so high that they can be toxic in the digester (Parkin and Owen, 1986). Heavy metals can also be toxic to anaerobic digestion. In Britain, heavy metals are the most important stress on the digesters (British Notes on Water Pollution, 1971). An excess of sulphide, that is concentrations of $200 \mathrm{mg} / \mathrm{L}$ or more, can also cause decreases in methanogenesis in anaerobic digesters (Rudolfs and Amberg, 1952). Other parameters which have been shown to cause toxic effects with high concentrations are certain organics, volatile acids, cations and ammonia-nitrogen (Parkin and Owen, 1986).

Previous studies on recovery/ reactivation of fecal coliforms have looked at different types of digesters: mesophilic, thermophilic and two-stage digestion schemes (Higgins and Murthy, 2006; Higgins et al., 2007). A mesophilic anaerobic digester has temperatures ranging from $27-38^{\circ} \mathrm{C}$ (Task Force on Anaerobic Sludge Digestion, 1987). Thermophilic reactors have temperatures ranging from $50-60^{\circ} \mathrm{C}$ (Parkin and Owen, 
1986). This type of reactor can achieve a much greater pathogen removal with a shorter retention time. A mesophilic reactor with an outflow of Escherichia coli $(E$ coli $)$ of $21 \mathrm{x}$ $10^{6} \mathrm{MPN} / \mathrm{g}$ DW (Most Probable Number / g Dry Weight) has been shown to achieve a decrease to $100 \mathrm{MPN} / \mathrm{g} \mathrm{DW}$ simply by switching to a thermophilic anaerobic digester (Oleszkiewicz and Mavinic, 2001). The anaerobic digester can be split into two phases. One way to split the digester is to separate the acid-phase and the methane production phase into two smaller units (Demirel and Yenigun, 2002). Temperature-Phased Anaerobic Digestion (TPAD), where mesophilic digestion follows thermophilic digestion, is another way to separate the digester into two phases. This method of stabilisation can reach a greater volatile solids reduction in a shorter time and is not affected by shock loading like single stage digesters can be (Santha et al., 2006). However, digested biosolids from TPAD systems can be more difficult to dewater as there is an accumulation of colloidal-sized proteins and polysaccharides which can consume a lot of polymer (Bivins and Novak, 2001).

\subsection{Conditioning}

After stabilisation, the levels of pathogens have been lowered to a safe level (typically below $2 \times 10^{6}$ fecal coliforms/ $\mathrm{g} \mathrm{DW}$ ) and the emphasis is then placed on reducing the volume of the biosolids. The role of conditioning is to increase the efficiency of the dewatering technique, this can be done through either physical or chemical conditioning (Dentel, 2001). For the purpose of this project, only chemical conditioning will be considered. Chemical conditioners can be either organic or inorganic in nature. In sludge treatment, organic polymers are typically used, they are compounds created through a 
large number of monomer chain reactions, these monomers can be either linear or branched (Dentel, 2001).

The molecular weight of these polymers can be very high, with molecular weights reaching up to 30 million $\mathrm{g} /$ mole containing over 100,000 monomers (Dentel, 2001). These polymers can carry a negative, positive or a neutral charge (Spellman, 1997). The polymer added to the wastewater sludge usually carries a positive charge as the organic molecules produced by microbes and the extracellular proteins carry a negative charge and the sludge can thus be neutralised (Higgins et al., 2006). The optimum polymer dose is usually found when charge neutralisation of the sludge has occurred (Dentel et al., 2000). For charged polymers, the degree of their charge is determined to a certain extent by the number of monomers on the chain (Dentel, 2001). The longer the chain of charged monomers is, the higher the degree of charge of the polymer.

As will be seen in the dewatering section, the amount of shear added to the system from mechanical dewatering can be substantial and this shear can lead to the deterioration of the sludge. The sludge is then more difficult to dewater and requires a higher polymer dose to overcome the effects of the shear to the system (Novak and Lynch, 1990). Certain polymers withstand shear more than others. Polymers with a higher molecular weight can enmesh the particles as well as neutralise the sludge. They condition not only by charge neutralisation but also by a mechanism called bridging (Dentel, 2001). Polymers with higher molecular weights can thus withstand shear better than those with relatively low molecular weights (Higgins et al., 2006). 
Polymers are available in different physical forms. There are three main forms with differences that affect their transportation, storage and make-up methods (information from Dentel, 2001). Liquid polymer, which is shipped in its workable state, is expensive to ship because of its high volume. The concentration of the polymer is usually between 4 to $9 \%$. The second physical form is the liquid emulsion product which is concentrated from 30 to $45 \%$ and has a lower viscosity than the water-based solution. It must be mixed with water to reach its workable state. The dry forms of polymer are the most difficult to make-up their solution but have the advantage of being the easiest to ship and store. In this study, both dry polymer and emulsion polymer were used.

\subsection{Dewatering}

Dewatering is the process that typically follows conditioning. Conditioning the sludge at its optimum dose prior to dewatering leads to a good solids recovery and a higher cake dryness (Task Force on Sludge Conditioning, 1988). Mechanical sludge dewatering

methods include vacuum filtration, pressure filtration, belt press filtration and centrifugation whereas natural methods include, sand drying beds and lagoons (Task Force on Sludge Dewatering, 1983).

The two most popular ways to dewater are by belt press filter and by centrifugation. For this project, the emphasis will be placed on centrifuge dewatering but belt press filtering will also be examined. Depending on the characteristics of the sludge being dewatered, one of these methods can be preferred over the other. In contrast to the work by Bivins 
and Novak (2001), it has been stated that two-phase digestion, either thermophilic/ mesophilic or acid phase/ gas phase anaerobic digesters can produce sludges that can dewater at a higher rate (Novak, 2006). The cake solids in the belt filter presses and the low-solids centrifuges are similar (US EPA, 1982), although newer high-solids centrifuges can achieve higher levels of cake solids than those described in the USEPA document from 1982. Sludges that can achieve higher solids contents can be dewatered with a low-pressure device whereas for harder to dewater sludges, considerable energy needs to be put into the dewatering (Novak, 2006). Dewatering performance can be measured through the solid recovery, the percent solid in the cake, the quality of the filtrate/ centrate and the polymer requirement for the process.

\section{Solid bowl centrifugation}

In solid bowl centrifuging, a centrifugal force separates the two phases (solid and liquid). Centrifuge dewatering can be done by either a low-solids centrifuge or a high-solids centrifuge. Although the high-solids centrifuge can achieve higher cake solids (in the range of 30 to $35 \%$ ), it does create extensive shear compared to the low-solids centrifuges and much more than other dewatering techniques such as a belt filter press (Novak, 2006). A solid bowl centrifuge can be continuously operated. It operates at a high relative centrifugal force which causes the solids in the sludge to settle towards the outside of the bowl and to then be carried by a screw conveyor to the outlet (Spellman, 1997). The centrate, on the other hand, flows into channels inside the dewatering beach to then be returned to the feed end of the machine (US EPA, 1982). A countercurrent full-scale 
centrifuge is pictured in Figure 1.1. In this type of centrifuge; the feed enters through the center of the bowl instead of the beginning of the beach.

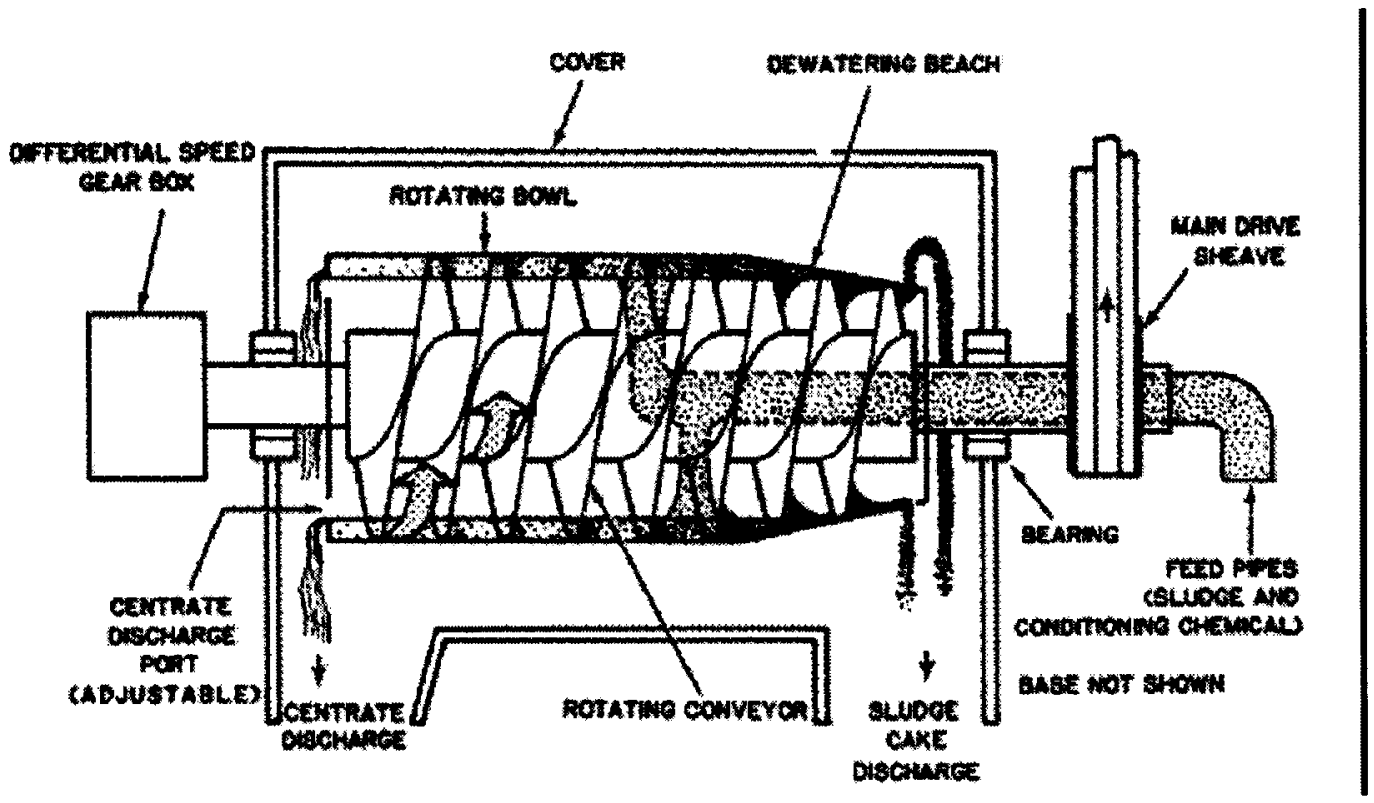

Figure 1.1: Countercurrent solid-bowl centrifuge (Source: US EPA, 1982)

At the sludge inlet, a high-solids centrifuge has approximately 10 times more shear than a belt filter press, because of this increase in shear, the polymer dose for centrifuges is four times higher than for a belt filter press (Novak, 2006). Polymer conditioning seeks to decrease the deterioration of the sludge due to shear so that the sludge can still dewater effectively (Novak and Lynch, 1990).

\section{Belt filter press}

Dewatering using a belt filter press adds less shear to the system. Also, in contrast with centrifuge dewatering, it has a lower power requirement, and makes less noise and less 
vibrations (US EPA, 1982). There are three phases to belt filter press dewatering as shown in Figure 1.2. Stage 1 is the chemical conditioning. Polymers are added in Stage 1 in order to improve Stage 2, the free drainage phase (Novak, 2001). The shear and compression phase is Stage 3. If the sludge is improperly dewatered, there could be blinding of the filter media and a loss of efficiency of the process at this stage (US EPA, 1982). A belt filter press does not add as much shear because it simply applies pressure to the sludge which is between two layers of cloth. It usually requires less polymer. On the disadvantage side, the process is very sensitive to changes in the sludge characteristics and requires a large quantity of wash water (Spellman, 1997).

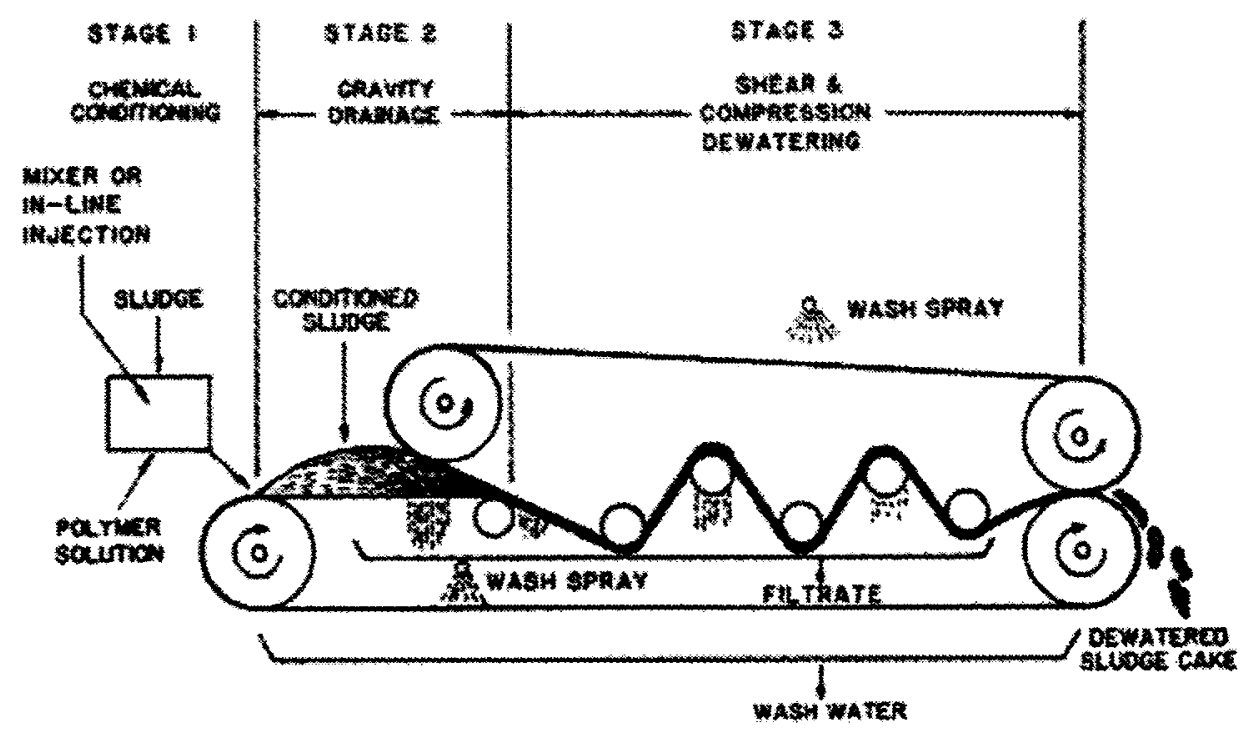

Figure 1.2: Belt filter press (Source: US EPA, 1982) 


\section{Thermal Drying: Pelletisation}

After dewatering, sludge can either go for final disposal or it can be dried and turned into pellets with solid concentrations between 92 and 98\% (Oleszkiewicz and Mavinic, 2001). On top of having a high solid concentration making it easier to ship, the pellets have extremely low pathogen counts as the dryers reach temperatures above those required for pasteurisation (Weemaes and Verstraete, 2001). The pellets which come out of the dryers have average diameters of $2 \mathrm{~mm}$ and can be used either as fertilizer or as a fuel source (Weemaes and Verstraete, 2001). Of the two treatment plants studied in this thesis, Treatment Plant B uses thermal drying on a portion of their dewatered sludge producing pellets with solid concentrations of $95 \%$.

\subsection{Disposal}

Sludge disposal can account for $50 \%$ of the operating cost of the wastewater treatment plant (Vesilind and Spinosa, 2001). Reusing the stabilised dewatering sludge, or biosolids, as a fertilizer is generally the most cost-effective method of disposal. However, this method can only be undertaken if the biosolids meet the criteria set out by the governmental agencies of the city, province or country. Land application is currently the method of disposal for $50 \%$ of the biosolids produced in Canada, but this application takes place on less than $1 \%$ of Canada's agricultural lands (Canadian Water and Wastewater Association, 2003).

In order to be land applied, treated municipal sewage sludge, or biosolids, must meet certain criteria for pathogen counts as well as metal concentrations. Regulatory 
frameworks exist in Canada and the European Union but they are primarily based on existing legislature for the United States (Oleszkiewicz and Mavinic, 2001). In the Part 503 guideline, the US EPA outlines restrictions for the disposal and use of biosolids (US EPA, 1994). There are three classes of biosolids for which land application is allowed. The descriptions of the pathogen requirements for these classes are outlined in Table 1.1. Disposal restrictions exist for class B biosolids but Class A biosolids can be applied in small quantities for recreational use, they can also be given or sold to the public as organic fertilizer. Exceptional Quality (EQ) biosolids have the same pathogen requirements as Class A biosolids but have added conditions for the concentration of heavy metals and are thus even less restricted in their land application.

Table 1.1: Pathogen reduction requirements (Source: Nova Scotia: Environment and Labour, 2004)

\begin{tabular}{|c|c|c|}
\hline Exceptional Quality & Class A & Class B \\
\hline $\begin{array}{c}\text { Fecal Coliform: }<1000 \\
\mathrm{MPN}^{*} / \mathrm{g} \text { total solids (dry } \\
\text { weight) } \\
\text { OR } \\
\text { Salmonella: }<3 \text { MPN* } / 4 \mathrm{~g} \\
\text { total solids (dry weight) }\end{array}$ & $\begin{array}{c}\text { Fecal Coliform: }<1000 \\
\mathrm{MPN}^{*} / g \text { total solids (dry } \\
\text { weight) } \\
\text { OR } \\
\text { Salmonella: }<3 \mathrm{MPN} / 4 \mathrm{~g} \text { total } \\
\text { solids (dry weight) }\end{array}$ & $\begin{array}{l}\text { Fecal Coliform: }<2,000,000 \\
\text { MPN }{ }^{\star} \text { per gram of total } \\
\text { solids (dry weight) }\end{array}$ \\
\hline
\end{tabular}

Throughout the different provinces and territories in Canada, there is different legislation for sludge quality and the different disposal options that are available. In Ontario, there are regulatory tools to manage discharges into the environment but there are no restrictions for the use or disposal of biosolids. In Ontario, land application is encouraged over incineration or landfilling (Lewis, 2006). Nova Scotia is the only province which has a restriction on the disposal of biosolids, it has banned the landfilling of compostable 
products (Nova Scotia: Environment and Labour, 2004), which means that more emphasis needs to be placed on meeting the pathogen requirements in this province because the biosolids cannot be disposed of in a landfill.

Centrate has a very low solid concentration but can have high nutrient levels. It is typically returned to the start of the treatment train but its high levels of ammonia and phosphate can require a separate treatment process before it can be recycled to the beginning of the treatment plant. These separate processes can be expensive and require additional space at the treatment plant (Mossakowska et al., 1997). Its nutrient levels however, could make it an attractive fertilizer in arid countries where the high transportation costs would be justified because of the lack of freshwater for irrigation. Land disposal of centrate would have to meet the same pathogen requirements as those laid out for the land application of biosolids. Centrate disposal on land is currently prevented by the high concentrations of ammonia and nitrogen from the application. Tests have shown that concentrations of ammonia above $100 \mathrm{mg} / \mathrm{L}$ have been inhibitory for plant growth (Baumgartner et al., 2005). As centrate is very seldom land applied, nutrient concentrations and pathogen enumeration is rarely undertaken.

\subsection{Treatment plant data}

There are two treatment plants that will be examined in this study. The characteristics of the treatment trains for these two treatment plants in Ontario (names have been withheld upon request from plants) are given in Table 1.2. Treatment Plant $\mathrm{A}$ has a high solids centrifuge, the effect of using a high solid versus a low solid centrifuge will be discussed 
further in Chapter 2. Treatment Plant B used a low solids centrifuge and had pelletisation after dewatering; meaning the pathogens in the cake would not be an issue as they would be eliminated in the thermal drying process and increases in fecal coliforms following this process would be unlikely.

Table 1.2: Treatment train characteristics

\begin{tabular}{|l|c|c|c|c|}
\hline & $\begin{array}{c}\text { Stabilisation } \\
\text { method }\end{array}$ & $\begin{array}{c}\text { Dewatering } \\
\text { method }\end{array}$ & $\begin{array}{c}\text { Solids } \\
\text { obtained after } \\
\text { centrifugation }\end{array}$ & Other processes \\
\hline $\begin{array}{l}\text { Treatment } \\
\text { Plant A }\end{array}$ & $\begin{array}{c}\text { Mesophilic } \\
\text { anaerobic } \\
\text { digestion }\end{array}$ & $\begin{array}{c}\text { High solids } \\
\text { centrifuge }\end{array}$ & $30-35 \%$ & $\begin{array}{c}\text { Centrifuge } \\
\text { thickening pre- } \\
\text { stabilisation }\end{array}$ \\
\hline $\begin{array}{l}\text { Treatment } \\
\text { Plant B }\end{array}$ & $\begin{array}{c}\text { Mesophilic } \\
\text { anaerobic } \\
\text { digestion }\end{array}$ & $\begin{array}{c}\text { Low-solids } \\
\text { centrifuge }\end{array}$ & $18-20 \%$ & $\begin{array}{c}\text { Pelletisation } \\
\text { post-dewatering }\end{array}$ \\
\hline
\end{tabular}

Historical indicator organism data was obtained from Treatment Plant A. Data was collected immediately following digestion as well as after centrifuge dewatering. Sampling was undertaken at various times throughout the year and obtained for three consecutive years. Results for the solid concentration of the samples were not available and as such, typical solids concentrations of digested sludge and dewatered sludge cake were used to convert the data in terms of $\mathrm{cfu} / \mathrm{g} \mathrm{DW}$ (coliform forming units / gram Dry Weight). The data in Figure 1.3 is the average of approximately 30 sampling times with error bars showing standard deviations on the mean of the samples. Figure 1.3a is showing the raw data (given in terms of cfu's / $g$ wet weight) and Figure $1.3 \mathrm{~b}$ has the results for the data in terms of $\mathrm{cfu} / \mathrm{g} \mathrm{DW}$ with the assumptions made for the percent solids of each of the samples. What this figure shows, is that increases in $E$ coli are observed at a local treatment plant following anaerobic digestion and centrifuge 
dewatering and that these differences in the culture counts are significantly different (by using a paired t-test) for three consecutive years of sampling.
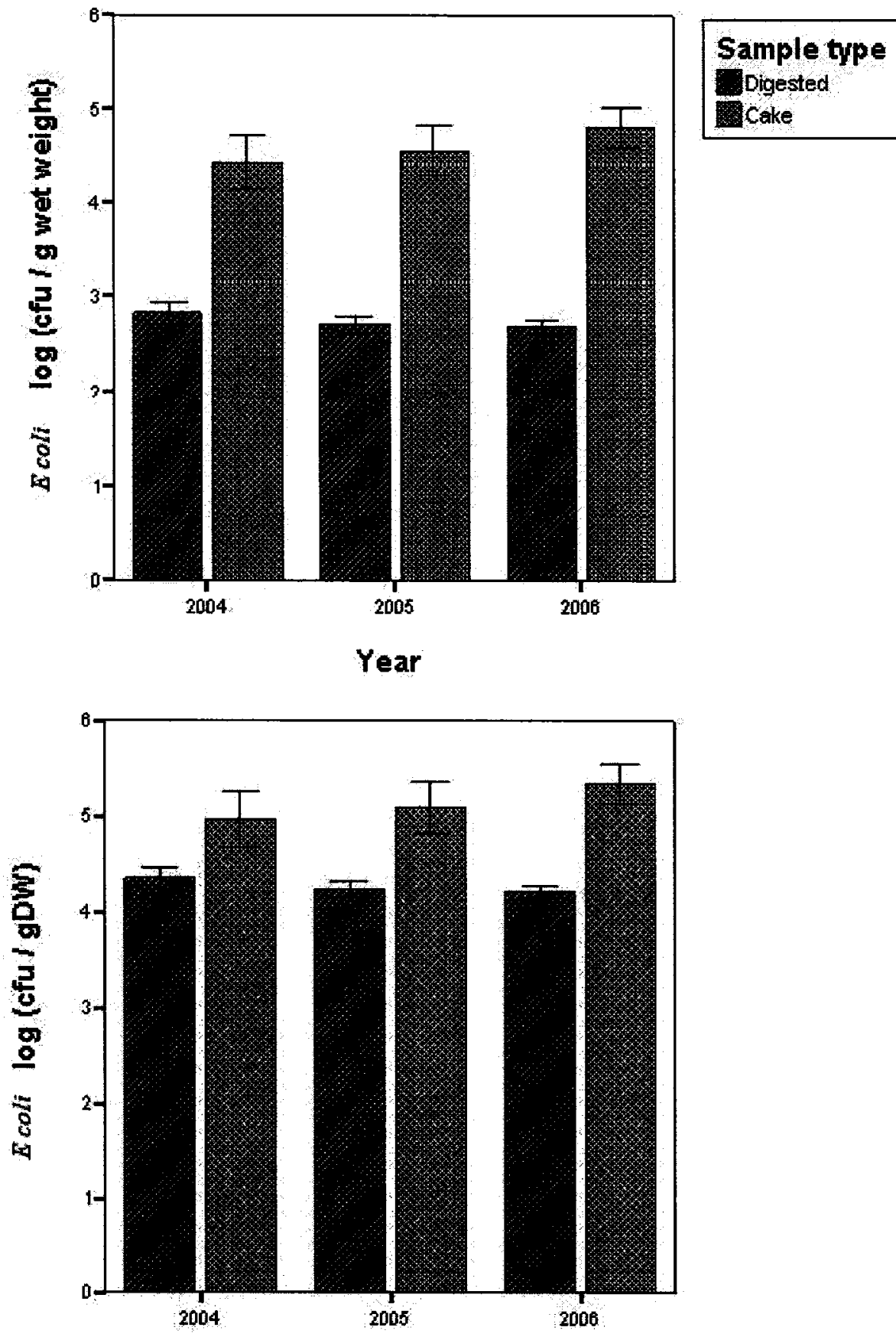

Figure 1.3: $E$ coli concentrations in digested sludge and dewatered sludge cake at Treatment Plant A. a) cfu / g wet weight obtained from the treatment plant, b) cfu / g DW calculated using average solids concentrations. Error bar are for standard deviations on the mean of the samples, $n=43$ for $2004, n=37$ for 2005 and $n=29$ for 2006 . 
Unfortunately, E coli or fecal coliform data could not be obtained for Treatment Plant B, but as dewatering is not their final process before disposal, it would not be necessary to measure indicator bacteria after centrifugation. Pathogen enumeration at the treatment plants is usually undertaken immediately following the stabilisation phase as the reduction in pathogens is achieved during this process. The processes that follow stabilisation have the goal of lowering the volume of sludge to be disposed of and are not expected to have an effect on the bacterial counts. Recently, studies have reported increases in indicator organisms such as fecal coliform and $E$ coli counts during or shortly after mechanical dewatering (Higgins and Murthy, 2006; Higgins et al., 2007; Iranpour et al., 2005; Iranpour and Cox, 2006; Iranpour et al., 2006; Jolis, 2006; Monteleone et al., 2004; Qi et al., 2004; Qi et al., 2007). The concern for this increase is that it could cause the biosolids to no longer be in compliance with regulations set out by the country for their limits of indicator organisms. As a consequence, the final disposal options would also need to be modified. Other implications for the health and safety of the public need to be considered as well if the dewatered sludge cake was land applied with these high levels of pathogens. In addition to the observations in the literature, the historical data from Treatment Plant A indicates that this issue has been observed by some local treatment plants as well. Through this research the fate of fecal coliforms through centrifuge dewatering will be examined. 


\section{Chapter 2}

\section{Literature Review}

In order to understand the problem of post-dewatering increases in indicator bacteria which was presented in Chapter 1 , it is necessary to look at: the background information, the conditions leading to these observations as well as the topics to be further studied in this paper.

\subsection{Background information}

The terms regrowth and reactivation are sometimes used interchangeably to describe the same results, they are in fact two distinct terms and will need to be described separately. The concept of Viable But Non Culturable Bacteria (from here on called VBNC) is closely related to the terms regrowth and reactivation, and as such, also needs to be described. Enumeration of indicator bacteria can be done by either culture-based methods or molecular methods. Comparing the values given by these two methods can often give a count of the VBNC bacteria. For this reason, a brief description of the culture methods and the molecular methods used in sludge treatment will be given.

\subsubsection{Regrowth / Reactivation}

For regrowth to occur, culturable background bacteria must be present. These background bacteria would be able to grow under ideal conditions; however, significant time is needed for them to increase their numbers by several orders of magnitude (Higgins et al., 2007). The doubling time of $E$ coli is 20 minutes. If the increase in the bacteria takes from hours to days to occur, the cause of the increase would be the growth 
of background organisms. When regrowth is observed, the increase in fecal coliform numbers often is significant even if the change from the pre-dewatering counts is smaller than one $\log (\mathrm{Qi}$ et al., 2007). Conditions that lead to regrowth are usually related to changes in temperature or the removal of stress to the environment (Jolis, 2006).

Reactivation of bacteria is a different term than regrowth and is used when the observed change in culturable bacteria is greater than what would be possible given the doubling time of the organism and the time frame of the increase. This 'reactivation' is used in microbiology as the transition of the cells from non-culturable to culturable (Higgins et al., 2007). The typical solids retention time in a dewatering centrifuge is only $10-25$ minutes, which is not enough time to observe some of the increases through the growth of the bacteria alone. In some studies, increases of several orders of magnitude were seen between the time of entry into the centrifuge and its exit from the centrifuge (Higgins et al., 2007; Qi et al., 2007). It is for this reason that the authors equate these changes in fecal coliform counts to reactivation instead of regrowth. Another term which is used occasionally with reactivation is resuscitation. In most papers, these terms are one in the same; only one group of authors distinguish the two terms. They state that reactivation is the return to culturability without supplementation, whereas resuscitation is the return to culturability following the addition of nutrients (Reissbrodt et al., 2002). Using these two definitions, the post-dewatering increases in indicator organisms would be due to reactivation as no nutrients are added to the system. 


\subsubsection{Viable but Non Culturable Bacteria}

The concept of VBNC bacteria is heavily debated within the microbiology community; much of the debate is related to the possibility of cells being capable of exiting this state. The debate will be briefly presented here. A more detailed discussion on VBNC bacteria is presented by Barer and Harwood (1999). A simple definition of VBNC bacteria is: cells which are metabolically active but incapable of undergoing the cellular division needed for bacterial enumeration on culture media (Mizunoe et al., 1999). For some researchers, culturability reflects viability and the only way to conceptually describe viability is through culturability (Barer and Harwood, 1999). Similarly, in this view, the loss of culturability is a step towards the eventual loss of viability. The metabolic activity that is observed is only temporary, before the cell becomes more debilitated (McDougald et al., 1998). Entrance into the VBNC state can also be viewed as a survival strategy of the bacteria when they are placed under stressful conditions. Once the stress is removed, the cells can be resuscitated from this state to become culturable again (Oliver, 1993).

The induction of cells into the VBNC state has been observed for cells which have been placed at low temperatures, $5^{\circ} \mathrm{C}$ (Rigsbee et al., 1997), as well as high temperatures: above $20^{\circ} \mathrm{C}$ (Adams et al., 2003) and at $37^{\circ} \mathrm{C}$ (Gauthier, 2000). The most important inducer however, has been said to be nutrient scarcity (Mckay, 1992). Toxicants, such as metals, chemicals and antibiotics are said to cause sub-lethal injuries and do not induce cells into the VBNC state (McDougald et al., 1998). 
The controversy over true resuscitation of VBNC cells comes back to the distinction between regrowth and reactivation. The apparent resuscitation of cells out of the VBNC state can often be confused with the growth or replication of the background culturable bacteria once the stressful conditions are removed (see definition of regrowth). Some research has shown increases in bacteria by so many orders of magnitude and in such a short time, that the authors ruled out regrowth from background cells claiming that the doubling time for the background cells was not sufficient and that this increase was due to the reactivation of VBNC bacteria (Bjergbaek and Roslev, 2005).

In contrary to the studies of Bjergbaek and colleagues (2005), in other studies, increases in culturable bacteria are only possible when background culturable bacteria is present. Further, their tests have shown that the culturable bacteria increased to the same level even with different dilutions. Had it been reactivation of VBNC bacteria, the more diluted sample would have less bacteria (Ravel et al., 1995). In some cases, growth is observed for cells when there is no apparent change in the conditions, this is called cryptic growth (Barer and Harwood, 1999). This concept has been associated with the release of nutrients through cell lysis after the cell's death (Na et al., 2006). The release of the nutrients from the membranes of the dead cells can be taken up by other bacteria and cause an apparent growth in the population (Arana et al., 2004). 


\subsubsection{Enumeration methods}

\section{Membrane Filtration and plating methods}

Membrane filtration is a culture-based method used commonly in water and wastewater treatment. Methods for the detection of fecal coliforms are described in Standard Methods: (American Public Health Association (APHA), American Water Works Association and Water Pollution Control Federation, 1989). For membrane filtration, a volume of the diluted sample is filtered through a $0.45 \mu \mathrm{m}$ pore size filter and then the filter is laid on a petri dish containing media for the detection of the indicator organism in question. After incubation, the number of colonies visible on the filter are counted and the results are given in terms of colony forming units (cfu) per $\mathrm{ml}$ and are usually reported based on the solids content of the sample to give numbers in terms of cfu/g DW. Plating is another culture based method which is similar to membrane filtration but involves the spreading of the diluted sample on a media plate. UK Water Industry Research recommends using a plating medium for the detection of indicator bacteria in water and wastewater (Eccles et al., 2004).

\section{Multiple Tube Fermentation}

Multiple Tube Fermentation (MTF) is also known as the Most Probable Number (MPN) method as the results are given as MPN / $\mathrm{g}$ wet weight. As done for the methods above, it is reported as MPN / $\mathrm{g}$ DW. The US EPA recommends using the MPN method for the detection of indicator microorganisms in sludge or biosolids. Two MPN methods are used in this project and will be explained in more detail in Section 4.1.6 (US EPA, 2005a; 
US EPA, 2005b). The MPN method has a slight advantage in that it's most probable number is not limited by the definition of zero viability that exists for the colony counting methods (Kell et al., 1998).

\section{$\underline{P C R}$}

Polymerase Chain Reaction (PCR) is a molecular method used for the detection of viable cells. PCR counts can be compared with culture counts in order to obtain the amount VBNC bacteria in the sample. Researchers have used this method to compare the $E$ coli counts before and after dewatering by centrifugation (Higgins and Murthy, 2006; Higgins et al., 2007). When using PCR, a good recovery of Deoxyribonucleic acid (DNA) is important. Two methods are typically used for sludge and biosolids, either competitive PCR (cPCR) or reverse transcriptase PCR (rt-PCR). The rt-PCR method is more consistent and provides a greater sensitivity than $\mathrm{cPCR}$ however, $\mathrm{cPCR}$ is simple to run and does not require the expensive system that rt-PCR requires (Chen et al., 2006). It has been possible to use it-PCR to detect mitochondrial DNA from samples which had less than 50 viable cells in them (Lleo et al., 2001). The low sensitivity is very positive; however the method has been shown to overestimate cell counts by including nonviable and unprotected DNA cells (Artz et al., 2006). Even those who use the method see its disadvantages, it has been said that if $E$ coli were beginning to die-off, the PCR method would continue to count them as cells because their DNA would remain intact (Higgins and Murthy, 2006). This would also lead to higher than expected numbers for the concentrations of the viable cells. 
Chen et al., (2006) outlined a method using cPCR which had similar sensitivity to rt-PCR all the while being less time-consuming than the rt-PCR method and giving a good recovery for DNA in solid samples of sludge and biosolids. It is the belief of some researchers that PCR is qualitative and that it suffers because it needs to be coupled with another viability method for its final verification (Artz et al., 2006). The methods used by Higgins, Chen and colleagues are said to be qualitative (Higgins et al., 2007).

\subsection{Conditions of observed regrowth / reactivation}

Differences in the sludge treatment plant characteristics have been shown to have an effect on the regrowth / reactivation of indicator organisms. Differences in the temperature of the anaerobic digesters, the change in temperature post-digestion, and most importantly the choice of mechanical dewatering method can all have an effect on the post digestion fecal coliform concentrations. The conditions that lead to a greater increase or those that would lead to reactivation as opposed to regrowth will be presented in the sections below.

\subsubsection{Centrifuge versus belt press dewatering}

The choice of the mechanical dewatering method has the greatest effect on possible increases in fecal coliforms. Increases have only been observed following centrifuge dewatering and not after belt filter press dewatering. The first few studies dealing with the subject of reactivation and regrowth of bacteria compared the levels of fecal coliforms before and after dewatering by centrifuge as well as with a belt press filter 
(Monteleone et al., 2004; Qi et al., 2004). In both these papers, the fecal coliform or $E$ coli levels in the cake after belt press filtering either stayed the same (no significant change) (Qi et al., 2004) or showed a decrease (Monteleone et al., 2004). Increases were observed after centrifuge dewatering as well as after aging the cake samples at room temperature (Monteleone et al., 2004; Qi et al., 2004). These increases were not universal for all treatment plants using centrifuge dewatering nor were they consistent on different sampling days at the same plant.

As these authors saw an increase only after centrifuge dewatering, they hypothesised that the increase could be due to the shear which is introduced to the system in centrifuge dewatering which is not as pronounced in belt filter press dewatering. The shear added to the system could cause the flocs of bacteria to break up and release bacteria leading to higher counts from standard culture methods. In most cases, samples are homogenised in a blender to ensure a uniform sample before enumeration. This step would add shear to all samples and could possibly increase the counts of fecal coliforms by breaking up flocs of bacteria. Laboratory tests where sludge was blended for up to 20 minutes showed no change in the fecal coliform counts during the extent of the testing period (Qi et al., 2007). The same study also looked at the effect of a sonic disperser device which also did not show any increase. In contrast to the studies on the effect of shear during enumeration, very little work has been done on the examination of the shear added directly from the centrifuge during dewatering. 


\subsubsection{High-solids versus low-solids centrifuge}

As seen in Section 1.4, high-solids centrifuges introduce even more shear than low-solids centrifuges. Studies have shown increases in indicator organism counts using both types of centrifuges, however, the increase was greater and more likely to be significant when a high-solids centrifuge was used (Qi et al., 2004; Qi et al., 2007). In other studies, highsolids centrifuges were used exclusively (Iranpour et al., 2005; Iranpour and Cox, 2006; Iranpour et al., 2006; Monteleone et al., 2004); this type is used more often in full-scale plants as it leads to a higher cake solid concentration and because it is more efficient.

\subsubsection{Change in temperature post digestion}

Changes in temperature have been shown to lead to the growth of background cells as well as the return to culturability of VBNC bacteria. VBNC bacteria were shown to resuscitate when the cells were taken from an environment at $5^{\circ} \mathrm{C}$ and put at $25^{\circ} \mathrm{C}$ (Oliver et al., 1995). In the case of the studies on increases after mechanical dewatering, many of these increases have been seen after the samples were 'aged' at optimum temperatures, meaning the placing of these samples at these temperatures (room temperature or incubation temperature) for a certain amount of time.

Much of the work on the change in post-digestion temperature has been done by Iranpour and colleagues (Iranpour et al., 2005; Iranpour et al., 2006 and Iranpour and Cox, 2006). The coliforms exiting the thermophilic digester were at a temperature above $50^{\circ} \mathrm{C}$, which is near pasteurisation temperature. After exiting, the fecal coliforms were below the Class 
A limit and remained low throughout the high-solids centrifuge dewatering and the conveyor belt. However, at the truck loading facility an increase was seen, raising fecal coliform levels to above the Class A limits (Iranpour et al., 2005). In the silo, where the biosolids (or dewatered sludge cake) were stored before application on land, the temperature was measured as $41^{\circ} \mathrm{C}$. At the truck hauling station, the increase from the post-digestion numbers was of four log (Iranpour et al., 2006). The authors also spiked samples of digested sludge as well as dewatered sludge cake and aged the samples for four days at 25,35 and $45^{\circ} \mathrm{C}$. For all temperatures, the dewatered cake showed an increase whereas the digested samples all decreased (Iranpour and Cox, 2006). It can therefore be stated that it is a combination of the drop in post-digestion temperature and the characteristics of the dewatered sludge that allow for the regrowth at that treatment plant.

After 24 hours of aging, Qi et al., (2004) saw a greater increase in fecal coliform numbers. Further studies by these researchers showed that after 20 days at both $25^{\circ} \mathrm{C}$ and $37^{\circ} \mathrm{C}$, the samples of dewatered sludge had the chance to increase and then decrease but that at the end of the sampling time, the fecal coliform levels were still two orders of magnitude higher than the original levels (Qi et al., 2007). A similar test was undertaken by Jolis (2006); in this case, samples were placed at 20,35 and $50^{\circ} \mathrm{C}$. Only two sludge types showed regrowth and this regrowth was only seen at $35^{\circ} \mathrm{C}$, both other temperatures had the fecal coliforms remaining at their low or undetectable levels (Jolis, 2006). It was hypothesised that a well-digested sludge could not lead to regrowth as all the cells would be dead or damaged beyond repair as opposed to other digester environments where 
culturable or viable bacteria remained. As none of the increases in the cell counts were significant before 48 hours of aging, the author also stated that reactivation within the treatment plant was unlikely (Jolis, 2006).

\subsubsection{Type of anaerobic digester}

As seen in Section 1.2, anaerobic digestion can be undertaken at different temperatures. Increases in fecal coliforms have been seen after centrifuge dewatering of mesophilic, thermophilic and TPAD digested sludges. The Water Environment Research Federation (WERF) study by Higgins and Murthy (2006) looked at seven different treatment plants with different types of anaerobic digestion. In the study, increases in $E$ coli were observed in four of the treatment plants, one TPAD, one thermophilic and two mesophilic (Higgins and Murthy, 2006). The increases immediately following digestion were in the order of one to two log for the mesophilic digesters whereas four to five log differences were seen for the thermophilic and TPAD digesters. The levels of fecal coliforms exiting these last two digesters are much lower than those exiting the mesophilic digesters. It is the lower pathogen counts achieved in thermophilic digestion that are the basis of their appeal in the market. Most thermophilic digester effluents achieve class A standards with a shorter solids retention time than a mesophilic digester. If the solids retention time in the thermophilic digester is increased, no reactivation or regrowth of indicator organisms is seen (Higgins and Murthy, 2006). It would seem that the bacteria are in the VBNC state as they can be detected using PCR technology even if they cannot be detected by culture methods. After dewatering, the PCR cell counts do not change, whereas the culturable counts increase significantly (Higgins and Murthy, 2006). 
In all studies, if an increase after mesophilic digestion is seen and is significant, the increases are not as considerable as those following thermophilic digestion. It has been said that mesophilic digestion leads to the regrowth of the indicator bacteria and not reactivation. Although significant, the increases were often of less than one order of magnitude (Qi et al., 2007). The combination of thermophilic digestion and a cake with a high solids concentration led to the largest increase in fecal coliforms, an increase of over six $\log ($ Qi et al., 2007).

\subsection{Topics to be examined further from current study}

The previous sections of the literature review chapter have focused on the observations made by other researchers in their studies. As well, Section 1.6 showed that at least one of the treatment plants used in the current study has historically seen increases in fecal coliforms following dewatering with a high-solids centrifuge. The research in the field of regrowth or reactivation of fecal coliforms post-dewatering is still new; only a handful of papers have been written, and these are all within the last three years. Although more treatment plants are recognizing that increases can occur, these increases are by no means universal. There is still much work that needs to be done on the subject and the following sections will outline the main topics for which testing or research is lacking. The objectives of this study, outlined in Section 2.4, are to examine these topics and test the hypotheses in Chapter 3. 


\subsubsection{Centrifugation shear}

The shear introduced during the extraction procedure of bacterial enumeration has been identified previously as a possible cause for the reactivation / regrowth (Section 2.2.1). However, these studies looked into the shear from the extraction process. During the extraction of the bacteria all samples are put through high shear environments as they are typically homogenised in the blender. Blending was examined as a possible cause for increased coliform counts because of the breaking up of flocs (Higgins et al., 2007; Qi et al., 2007). Both studies mentioned did not see a rise in fecal coliform counts after blending. The shear introduced during centrifugation has not been examined in detail but as with shear that is introduced during the extraction procedures, it could cause flocs of bacteria to break up and release more bacteria into the environment, making fecal coliform counts appear higher.

Shear could also negatively affect cell counts by modifying cell surface parameters by decreasing the surface charges of cells (Pembrey et al., 1999). High shear environments have also been associated with damage to the cell membranes making the cells more susceptible to external influences (Gilbert et al., 1991). Even low speed centrifugation, up to 3000 revs per minute (RPM), for 5 minutes has been shown to decrease total coliform counts (Örmeci and Linden, 2005). Culturability of cells has also been affected by the shear associated with centrifugation. At forces of $15000 \times \mathrm{g}$ (acceleration due to gravity), culturable $E$ coli dropped significantly (Pembrey et al., 1999). At $5000 \times \mathrm{g}$ for 5 minutes, viability was measured to be $80 \%$ of its original value (Gilbert et al., 1991). 
Thus, shear may increase or decrease the fecal coliform counts depending on its intensity, the sensitivity of the microorganism, and the sludge characteristics. The effect of shear can be modified by the presence of extracellular polymers (Örmeci and Linden, 2005) as well as the addition of synthetic polymers to the sludge. This will be examined further in the following section.

\subsubsection{Conditioning with polymers}

During centrifugation, the high shear and the centrifugal acceleration of the sludge could lead to polymer being stripped off of particles, requiring more polymer but also releasing bacteria within the floc and thus generating higher counts. It could also damage bacteria when the polymer is being ripped off leading to lower counts post-centrifugation.

The choice of polymers to be used during conditioning may impact the fecal coliform counts as well. The differences in the molecular weight, cationic charge, chain length, structure, and functional groups attached to the polymers are likely to influence the aggregation of bacteria and their attachment to sludge solids. The very first step of bacterial enumeration using culture methods is the extraction step. Extraction is the detachment of microorganisms from particles using physical (e.g., blending, homogenisation, centrifugation, sonication) and chemical methods (e.g., EDTA, EGTA, Zwittergent 3-12, Tris buffer, peptone) (Örmeci and Linden, 2005). Biopolymers have been shown to impact the extraction efficiency and final counts of bacteria. The presence of extracellular polymers result in bacteria having stronger bonds with particles (Prieme 
et al., 1996). Polymer bridging and hydrogen bonding can lead to stronger bonds between the bacteria and the particles (Stotzky, 1985) making the extraction of the bacteria more difficult and thus affecting the final culture counts.

Synthetic polymers, which typically have a very high molecular weight and carry a positive charge (Dentel, 2001), may have a larger impact on the extraction efficiency of bacteria from particles. Therefore, polymer type and dose used during conditioning may have an impact on fecal coliform counts measured after dewatering using culture methods. The possibility of the addition of polymers as having an effect on fecal coliforms was briefly examined by Iranpour et al., (2005) but the results remain unpublished and very little is known on the experimental procedure. What was stated is that they used a lab-scale centrifuge which only reached a maximum solid concentration of $10 \%$ (Iranpour et al., 2005). It is perhaps because of the low solids content from the centrifuge they used that no change in fecal coliform counts was observed.

Polymers may also serve as a substrate source for bacteria and could promote reactivation and regrowth. These polymers are biodegradable and can serve as carbon and nutrient sources for the cells (Chang et al., 2001). The carbon is found on a long chain and would therefore be less likely to be utilisable as a nutrient source. The nitrogen from the amide groups found on the polymer chain could be used though (Haveroen et al., 2005). Nitrogen is well-documented to be utilised for growth by coliform bacteria (Morrison and Hinshelwood, 1949). It has been shown that adding polymer to digested sludge can 
increase the fecal coliforms levels significantly, the more polymer added, the bigger the increase (Qi et al., 2007).

\subsubsection{Viable versus culturable counts}

Work by Higgins and colleagues has shown that decreases observed in the culturable bacteria from anaerobic digestion, either mesophilic or thermophilic, do not always lead to a decrease in the viable cell counts (Higgins and Murthy, 2006; Higgins et al., 2007). Although the culturable bacteria counts decrease after digestion and then increase after centrifugation, very little changes are seen in the viable bacteria observed by the results of the cPCR. Other studies have shown that bacteria in sludge can enter a VBNC state (Pacheco et al., 2003; Wery et al., 2006).

When the increases in the post-dewatering culturable counts are faster than the doubling time of the bacteria, the increases cannot be due to regrowth alone and another mode of increase is necessary. Other hypotheses explored in papers include the contamination from the centrifuge; as solids get pushed to the peripheries they could accumulate there. Therefore, the solids coming out of the centrifuge could have resided in the centrifuge for much longer than the average retention time of 15-20 minutes and thus the growth of fecal coliforms would be possible. Although it has been examined as a possibility for large increases in a short time period (Jolis, 2006), it is unlikely that contamination alone could be the cause of a four log increase within the centrifuge as was seen in the work by Higgins et al. (2006 and 2007). 
In order to observe any discrepancies between the fate of fecal coliforms as compared to the total cell population post-dewatering, the culturable bacteria will need to be enumerated along with the viable bacteria in samples pre and post dewatering. The fecal coliform levels in the sludge need to increase to a sufficient degree so that regrowth can be ruled out.

\subsubsection{Centrate characterisation}

Centrate has been briefly examined in previous literature as a growth inhibitor as well as a growth promoter. Qi and colleagues tested their centrate for its possibility as a growth inhibitor. Their test used centrate produced in the lab and saw no effect concluding that it was not a growth inhibitor (Qi et al., 2007). However, it would be more indicative to test the full-scale centrate as it is in the full-scale environment of the treatment plant that these increases were observed. No increases in fecal coliforms from the laboratory produced cake were observed and thus the lab-scale centrate would not necessarily show the presence of a growth inhibitor.

The WERF report (Higgins and Murthy, 2006) examined centrate as a growth promoter. It is stated that high-solids centrifugation leads to a decrease in competition for the substrate and thus the growth of bacteria is promoted. Small volumes of centrate and polymers from one treatment plant (TPAD-1) were added to two thermophilically digested sludges from different treatment plants. It was hypothesised that the centrate from TPAD-1 showed 'autoinducer' type activity (Higgins and Murthy, 2006). What should be noted from these tests is that each treatment plant has different characteristics 
and this full-scale centrate characterisation will vary at the different plants as well. Although increases were observed, these were not of fecal coliforms or $E$ coli. What did increase was the Heterotrophic Plate count (HPC). HPC counts are not related to fecal coliform changes, as well, they do not necessarily lead to detrimental effects to human health (Allen et al., 2004). More research would need to be done in order to investigate the autoinducer activity of centrate.

It could also be that a substance in the digested sludge inhibits the growth of the bacteria and that during centrifugation, that substance is removed, allowing for the fecal coliforms in the sludge to increase. In a digester environment, many substances have been identified as inhibitors; some of them were briefly outlined in Section 1.2. Although much work exists on these toxic elements within the digester, very little work has been done on the possibility of inhibitory substances being released or removed during dewatering. A study in 1998 on the release of heavy metals post-centrifugation showed that increasing the centrifugal force on a laboratory centrifuge could lead to increases in the nickel concentrations in the centrate (El-Aassar et al., 1998). This could mean that the higher-solids centrifuges could possibly produce centrate with higher concentrations of inhibitory substances, such as nickel. This could be the reason why the removal of this centrate leads to post-dewatering increases in fecal coliform concentrations. The growth of the bacteria is no longer inhibited once the centrate is removed. The bacteria in the cake are no longer placed under stress leading to favourable conditions for regrowth or reactivation of viable cells. 
Although nitrogen can promote the growth of the bacteria; in high concentrations, it could also inhibit the growth of the fecal coliforms; it has been shown that centrifuge liquors have significantly higher concentrations of nitrogen than filtrate from belt filter presses (Thorndahl, 1993). High concentrations of some forms of Nitrogen could be the cause of the growth inhibition. Ammonia can reach toxic levels for the growth of plants (Baumgartner et al., 2005). Other forms which could be toxic are nitrate and nitrite. High levels of other nutrients could also be the cause of centrate as a growth inhibitor. The concentrations of certain species, such as nutrients in the centrate, could play an important role in bacterial growth inhibition.

\subsection{Objectives}

The goal of this research was to understand the reasons for the reactivation and regrowth of fecal coliforms after centrifuge dewatering. The following are the specific objectives for the research:

- To test the role that centrifugation shear can have on fecal coliform counts

- To examine the effect of different types and doses of conditioning polymers added prior to dewatering on the fecal coliform counts

- To investigate the role of growth inhibiting or promoting substances that may be present in sludge

- To compare the culturable fecal coliform with total viable cell counts to determine any discrepancies or agreements in these counts 


\section{Chapter 3}

\section{Hypotheses}

Research in the field of regrowth or reactivation of fecal coliforms is relatively new, only a handful of papers have been written, and these are all within the last three years. For this reason, not many hypotheses have been examined and very few causes for the increases in the fecal coliform counts post-dewatering have been tested.

In Section 2.3, topics related to the subject of post-dewatering fecal coliform regrowth which require further research were examined and the objectives of the research were laid out in Section 2.4. The following is a list of the hypotheses that will be examined; the next chapter will outline the experimental designs for the tests done in order to examine these hypotheses.

- Fecal coliform culture counts can be affected by the shear introduced from centrifuge dewatering which could cause the polymer to be ripped off of the sludge and help break up colonies of fecal coliforms. The characteristics of the conditioning polymer such as its cationic charge, its molecular weight and its physical form can also have an effect on culture methods.

- A toxin inhibiting the growth of bacteria can exist in the digested sludge and it can be removed in the centrate during centrifugation enabling reactivation or regrowth of fecal coliforms post-dewatering. 
- The culture counts of fecal coliforms may not necessarily represent viable bacterial counts. As well, the fate of fecal coliforms may not correlate with other microorganisms or pathogenic bacteria. 


\section{Chapter 4}

\section{Materials and Methods}

In order to test the hypotheses from Chapter 3, numerous methods need to be undertaken. The project was split into two different phases. The first hypothesis listed in Chapter 3 was examined through research done in Phase I of the project, whereas the two other hypotheses were examined through work done in Phase II.

Experiments from both Phases tested for the effect of aging the samples. This term is used often in the report and it refers to placing the samples for a certain number of days at a specific temperature and analysing the possible regrowth after this set amount of time. More information on the aging tests can be found in Section 4.2.1.2.

\subsection{Phase I}

Phase I of the project focused on the impact that the addition of polymer at different doses could have on bacterial counts. As well, it looked into the effect of the centrifuge speed. Comparing typical low-solids centrifuge speeds and high-solids centrifuge speeds and how these could affect the bacterial counts. All of these tests were undertaken using a laboratory centrifuge.

\subsubsection{Experimental design}

For Phase I, the experimental design is split into two tests; the centrifuge speed test and the laboratory polymer test. 


\subsubsection{Test 1: Centrifuge speed}

The first aspect that was to be tested was the effect of centrifuge shear on fecal coliform counts, studied by changing the centrifuge speed and an attempt to recreate the full-scale solid bowl centrifuge in the laboratory. Three different speeds were chosen which spanned the range of that would be seen for conventional (low-solids) and high-solids centrifuges (Monteleone et al., 2004). A speed of $2500 \mathrm{rpm}$ or a relative centrifugal force of 1300 times that of gravity $(1300 \mathrm{x} \mathrm{g})$ was chosen as a low-solids centrifuge surrogate. A speed of $3500 \mathrm{rpm}(2500 \mathrm{x} \mathrm{g})$ was chosen as being the border between what would be considered a high-solid and a low-solid centrifuge, typical low-solid centrifuges have accelerations in the range of 500 to $3000 \mathrm{x}$ (Task Force on Sludge Dewatering, 1983). Finally, a speed of $4500 \mathrm{rpm}(4140 \mathrm{xg})$ was chosen and had a centrifugal force slightly higher than would be seen in a high-solids solid bowl centrifuge.

Samples of anaerobically digested sludge were run through a Thermo Electron IEC Centra GP8 (Fisher Scientific, Toronto, ON) bench-scale, swinging bucket centrifuge which had a maximum rotor speed of $4850 \mathrm{rpm}$ or $4800 \mathrm{x} \mathrm{g}$. Samples were all run for 5 minutes; each sample was made up of $200 \mathrm{~mL}$ of digested sludge and placed in centrifuge jars into the swinging bucket centrifuge. After centrifugation, the samples were removed from the centrifuge and the centrate was poured out leaving the sludge cake in the bottom of the centrifuge jar. In full-scale high-solids centrifuges, separation of solids and centrate is done throughout the centrifuge dewatering and solids are retained for an average of 1520 minutes whereas the hydraulic retention times can be as low as 20 seconds (Vesilind et al., 1986). Although the hydraulic retention time for the laboratory centrifuges are 
much longer than the typical values for a full-scale centrifuge, the time of 5 minutes was chosen to achieve solids concentrations comparable to full-scale operation. Longer centrifugation times did not lead to solids concentrations which were much higher than those obtained after 5 minutes of centrifugation. Furthermore, longer centrifugation times would lead to changes in temperatures which could be causes of error for the fecal coliform counts.

Bacterial enumeration was carried out on the digested sludge prior to centrifugation as well as for the centrate or supernatant and the cake samples after centrifugation. Samples of digested sludge, centrate and cake were also placed at $24^{\circ} \mathrm{C} \pm 1^{\circ} \mathrm{C}$ for 48 hours and bacterial enumeration undertaken after 24 hours and 48 hours ( \pm 3 hours). Another run was undertaken; this time, polymer was added to the samples at its optimum polymer dose, determined as described in section 4.1.5. The same speeds, samples and bacterial enumeration were undertaken with this run of samples.

\subsubsection{Test 2: Polymer addition at different doses}

The second test looked into the effect of the type and dose of polymer being added. Digested sludge samples were run through the Thermo Electron IEC Centra GP8 centrifuge at $4500 \mathrm{rpm}$ for 5 minutes as this was the centrifuge cycle which gave the highest solids content. Samples were also placed at room temperature and fecal coliform enumeration was undertaken at 24 and 48 hours ( \pm 3 hours). Five different doses of polymer were chosen. The optimum dose, which was calculated in terms of $\mathrm{ml}$ of polymer per $200 \mathrm{ml}$ of sludge, was converted into $\mathrm{g} / \mathrm{kg}$ Dry Solid (DS). In addition to the 
optimum dose, two underdosed samples and two overdosed samples were also tested. Polymer was added to the sludge and mixed with paddle mixers at $300 \mathrm{rpm}$ for two minutes.

Even with the addition of polymer at the optimum dose and with the centrifuge cycle which gave the highest solids content, the maximum solids obtained from the samples was $13 \%$, which is much lower than typical cake solid concentrations in a high-solid or even a low-solid full-scale centrifuge. For this reason, a larger floor model centrifuge was used. This centrifuge also had the advantage of being refrigerated, eliminating the change in temperature with the centrifuge as a factor for changes in bacterial counts. A Sorval RC-5B centrifuge (Dupont, Toronto, ON) with the SLA-1500 rotor was used with a running time of 5 minutes, excluding acceleration and deceleration time at $10,000 \mathrm{rpm}$ $(15,180 \times \mathrm{g})$. This centrifuge had a fixed angle, after centrifugation the centrate was poured out of the centrifuge jar with the cake remaining at the bottom of the jar until the time of enumeration or sample preparation. The temperature during the centrifugation remained between $3-7^{\circ} \mathrm{C}$.

Bacterial enumerations were undertaken on digested samples, samples with polymer added but without centrifugation, as well as samples run through the centrifuge with or without polymer added. Centrate and cake were sampled separately. The cake and the centrate were decanted manually for the samples that had not been run through the centrifuge. Bacterial enumeration was also undertaken after 48 hours \pm 3 hours at room temperature. 


\subsubsection{Treatment plant characteristics}

Samples were collected from Treatment Plant A. An anaerobic digester is used to stabilise the waste activated sludge. The characteristics of the treatment plant are outlined in Table 4.1 and Section 1.6. Digested sludge from Treatment Plant A was exclusively used for the first phase of the experiment, with Treatment Plant B sludge being used in further testing outlined in Section 4.2. Both treatment plants use mesophilic anaerobic digesters to stabilise the waste activated sludge. Treatment Plant A had a larger capacity, used a high-solids centrifuge and thus produced a cake with a higher solids concentration. Treatment Plant B dewatered its digested sludge using a low-solids centrifuge and the average cake solids were $20 \%$. The polymers used by treatment plants were both Polyacrylamide powders mixed to solutions at the concentrations and at the doses outlined in Table 4.1 .

Table 4.1: Treatment plant flow, polymer type and dose

\begin{tabular}{|c|c|c|c|c|}
\hline & $\begin{array}{c}\text { Daily Flow } \\
\text { rate }\end{array}$ & $\begin{array}{c}\text { Polymer } \\
\text { type }\end{array}$ & $\begin{array}{c}\text { Polymer } \\
\text { dose }\end{array}$ & $\begin{array}{c}\text { Dewatering } \\
\text { method }\end{array}$ \\
\hline $\begin{array}{l}\text { Treatment } \\
\text { Plant A }\end{array}$ & $\begin{array}{c}460000 \mathrm{~m}^{3} / \text { day } \\
(121 \mathrm{MGD})\end{array}$ & $\begin{array}{c}\text { Flopolymer } \\
\text { CA4600 }\end{array}$ & $11 \mathrm{~g} / \mathrm{kg} \mathrm{DS}$ & $\begin{array}{c}\text { High solids } \\
\text { centrifuge }\end{array}$ \\
\hline $\begin{array}{l}\text { Treatment } \\
\text { Plant B }\end{array}$ & $\begin{array}{c}178000 \mathrm{~m}^{3} / \text { day } \\
\text { (47 MGD) }\end{array}$ & $\begin{array}{c}\text { FloPolymer } \\
\text { CB4350 }\end{array}$ & $8.4 \mathrm{~g} / \mathrm{kg} \mathrm{DS}$ & $\begin{array}{l}\text { Low-solids } \\
\text { centrifuge }\end{array}$ \\
\hline
\end{tabular}

\subsubsection{Anaerobically digested samples}

Samples were collected in the morning and transported to Carleton University for same day enumeration. All samples were kept at $4^{\circ} \mathrm{C}\left( \pm 0.5^{\circ} \mathrm{C}\right)$ until sampling time, which was 
within 6 hours of collection. Anaerobically digested sludge from Treatment Plant A had an average solids concentration of $2.5 \%$ whereas average solids concentrations at Treatment Plant B were 2\%. All samples were collected in a sterilised bucket and handled aseptically throughout the experiment.

\subsubsection{Polymers used}

Three polymers were used for the purpose of this study. Characteristics of the three polymers are given in Table 4.2. The Superfloc SD2061 and the FloPolymer CA4600 were used to examine the effect of the addition of polymer at different doses and the FloPolymer CB4350 was used in Full-scale sample testing from Phase II (Section 4.2.1.1). The main differences in the two polymers used in Phase I is their type as well as their molecular weight.

Table 4.2: Polymer characteristics

\begin{tabular}{llcc}
\hline Polymer name & Type of polymer & $\begin{array}{c}\text { Degree of cationic } \\
\text { charge (\%) }\end{array}$ & $\begin{array}{c}\text { Relative } \\
\text { molecular weight }\end{array}$ \\
\hline $\begin{array}{l}\text { Superfloc } \\
\text { SD2061 }\end{array}$ & $\begin{array}{l}\text { Emulsion } \\
\text { polyacrylamide }\end{array}$ & 40 & High \\
\hline $\begin{array}{l}\text { FloPolymer } \\
\text { CA4600 }\end{array}$ & $\begin{array}{l}\text { Polyacrylamide } \\
\text { Powder }\end{array}$ & 40 & Ultra High \\
\hline $\begin{array}{l}\text { FloPolymer } \\
\text { CB4350 }\end{array}$ & $\begin{array}{l}\text { Polyacrylamide } \\
\text { Powder }\end{array}$ & 15 & Ultra High \\
\hline
\end{tabular}

The emulsion polymer was obtained from Cytec Industries (West Paterson, New Jersey) with the jars of emulsion polymer kept between $20^{\circ} \mathrm{C}$ and $24^{\circ} \mathrm{C}$ until the polymer solution was prepared. Cytec Industries recommends a working solution of $0.5 \%$ and provide different methods for preparation of the working polymer solution. The make-up of all of the emulsion solutions used for this experiment was done as follows: $5 \mathrm{ml}$ of the 
emulsion solution was added to $1 \mathrm{~L}$ of deionised water. The solution was then blended using a hand blender (Cuisinart Quick Prep, Ottawa, ON) for 30 seconds. The solution was aged for at least 30 minutes at room temperature and new solutions were mixed for every new run of samples and were used within the day of testing.

The method for the make-up of the CA4600 dry polymer was quite different than the emulsion polymer. Two different runs were undertaken with the CA4600 polymer. For the first run, $3 \mathrm{~g}$ of dry polymer was added to $600 \mathrm{ml}$ of deionised water and stirred in a $1 \mathrm{~L}$ beaker for 60 minutes on a magnetic stir plate (Fisher Scientific, Toronto, ON). This was followed by blending with a hand blender for 5 to 10 seconds in order to break up the larger polymer flocs. The solution was then left to age for at least one hour at room temperature. The final concentration of this solution was $0.5 \%$. The second run was done with a polymer solution of $0.25 \%$; the method was the same except that only $1.5 \mathrm{~g}$ of powder polymer was used.

\subsubsection{Determination of the optimum polymer dose}

Two different methods were used to calculate the optimum polymer dose for the sludgepolymer combination for each run of samples. It was calculated by using a Capillary Suction Timer (CST) and a torque rheometer.

A Capillary Suction Timer (Venture Innovations Inc, Houston, TX) was used for quick optimum polymer tests; this method has been shown to be successful in dewatering testing to determine filterability of the sludge after the addition of polymers (Baskerville 
and Gale, 1968). Polymer was added to the anaerobically digested sludge and mixed with a laboratory spatula. The conditioned sludge was placed in the CST reservoir as per protocol in: (American Public Health Association (APHA), American Water Works Association and Water Pollution Control Federation, 1989). The time was calculated for an average of five different polymer doses and the shortest time was taken as the optimum dose.

The second way of determining the optimum polymer dose was by using a torque rheometer to study the peaks observed in unconditioned sludge immediately after the injection of polymer. The methodology used can be found as Method 1 in: (Örmeci, 2007). A $200 \mathrm{ml}$ sample of digested sludge is placed into the Floccky beaker of the Floccky Tester (Koei Industries Inc, CA) and the impeller is immersed into the sample. The sludge then goes through a three step process, step one has the sludge mixed at 420 rpm and step two is 40 seconds at $300 \mathrm{rpm}$. At this point, the polymer at the given dose is injected through the ports on the side of the Floccky beaker and for step three, the sludge is mixed at $300 \mathrm{rpm}$ for 120 seconds. The optimum polymer dose is taken as the dose which gives the highest peak on the Torque-time rheogram before a change in the trend of the rheograms is observed. 


\subsubsection{Bacterial enumeration}

Fecal coliform enumeration was undertaken using two different methods, both were multiple tube fermentation methods but using different media for the enumeration. The centrifuge speed aspect of Phase I of the project looked at fecal coliforms using the twostep verification method using Lauryl Tryptose Broth with EC medium (LTB / EC) for final verification. The protocol for this method is outlined in: (US EPA, 2005a). In order to compare the results obtained from the LTB/EC method with a quicker one-step verification method, the fecal coliforms in the samples from the centrifuge speed test were also quantified using A-1 medium with the protocol outlined in: (US EPA, 2005b). The remainder of the bacterial enumeration was done using the A-1 medium.

Homogenisation of samples was accomplished by blending using a Betty Crocker 12 Speed kitchen blender on high for 1 minute. Samples with solid concentrations above $7 \%$ were diluted tenfold by weighing out 30 grams of sample and homogenising it with 270 $\mathrm{ml}$ of phosphate-buffered solution (PBS). All cake samples had solids concentrations above $7 \%$ as well as some of the samples of digested sludge with polymer added prior to centrifugation. A minimum of three dilutions were used for each sample with five Durham tubes of prepared medium at each dilution. Each dilution was undertaken by taking $10 \mathrm{ml}$ of the least diluted sample and adding $90 \mathrm{ml}$ of PBS dilution water which was prepared following protocol in: US EPA, (2005b). 
Prepared tubes containing the A-1 media for the detection of fecal coliforms as well as the diluted sludge were placed in an incubator at $35^{\circ} \mathrm{C} \pm 0.5^{\circ} \mathrm{C}$ for 3 hours \pm 15 minutes followed by incubation at $44.5^{\circ} \mathrm{C} \pm 0.5^{\circ} \mathrm{C}$ for 21 hours \pm 3 hours. Tubes were then examined for gas production within the Durham tubes, inverted glass vials placed within the test tubes. The numbers obtained were placed into a Most Probable Number (MPN) calculator which would give the MPN by wet weight. Solids concentrations were obtained following protocol 2540B from: (American Public Health Association (APHA), American Water Works Association and Water Pollution Control Federation, 1989). Values of MPN per wet weight were then converted into MPN/g DW for comparison purposes.

\subsubsection{Statistical analysis}

Statistical analyses were undertaken using SPSS 13. Student t-tests, Analyses of Variance (ANOVA) tables and Least Significant Difference (LSD) post hoc tests were used for the determination of the significance of the differences between the individual bacterial counts.

\subsection{Phase II}

This part of the project focused more on the regrowth and reactivation of the fecal coliforms. For this phase, sludge, centrate and cake samples were obtained from the treatment plants themselves as opposed to generating the latter samples by centrifugation in the lab. It looked at the changes in fecal coliform counts within the treatment train as 
well as the role that the removal of centrate during centrifuge dewatering could have on bacterial counts.

\subsubsection{Experimental design}

The experimental design for this phase was split into five separate tests. The first test enumerated the fecal coliforms at different locations in the treatment plant. For the second test, samples and mixtures of cake and centrate were placed at different temperatures and enumeration was done on subsequent days to determine any changes in fecal coliforms with aging at these temperatures. The third test looked at the characterisation of the full-scale centrate by comparing the concentrations of its chemical constituents with those of lab-scale centrate samples. The fourth test involved the seeding of sterilised full-scale and lab-scale centrate with known fecal coliforms cultures and examining their growth and decline to gauge the toxicity of the samples. For the fifth test, the cellular ATP (Adenosine Triphosphate) in the centrate samples was measured to quantify the viability of bacteria as well as their relative toxicity. The experimental designs for each of these are given in the following sections, followed by more detailed sections for specific materials and methods for this phase of the project.

\subsubsection{Test 1: Full-scale treatment plant}

The goal of this test was to examine the fate of fecal coliforms throughout the sludge treatment train. Comparing the densities of the bacteria before and after each treatment process enabled the examination of any changes in fecal coliform densities throughout the treatment train. Samples were collected at various locations at the wastewater 
treatment plants and bacterial enumerations were undertaken throughout the treatment train. Two separate treatment plants were examined. Characteristics of both treatment plants have already been described in Table 1.2 and Table 4.1 of this document. Sampling locations for both treatment plants were the same and are laid out in Figure 4.1. Treatment Plant B had an extra sampling location after dewatering as some of their digested sludge is thermally treated and high solid pellets are produced.

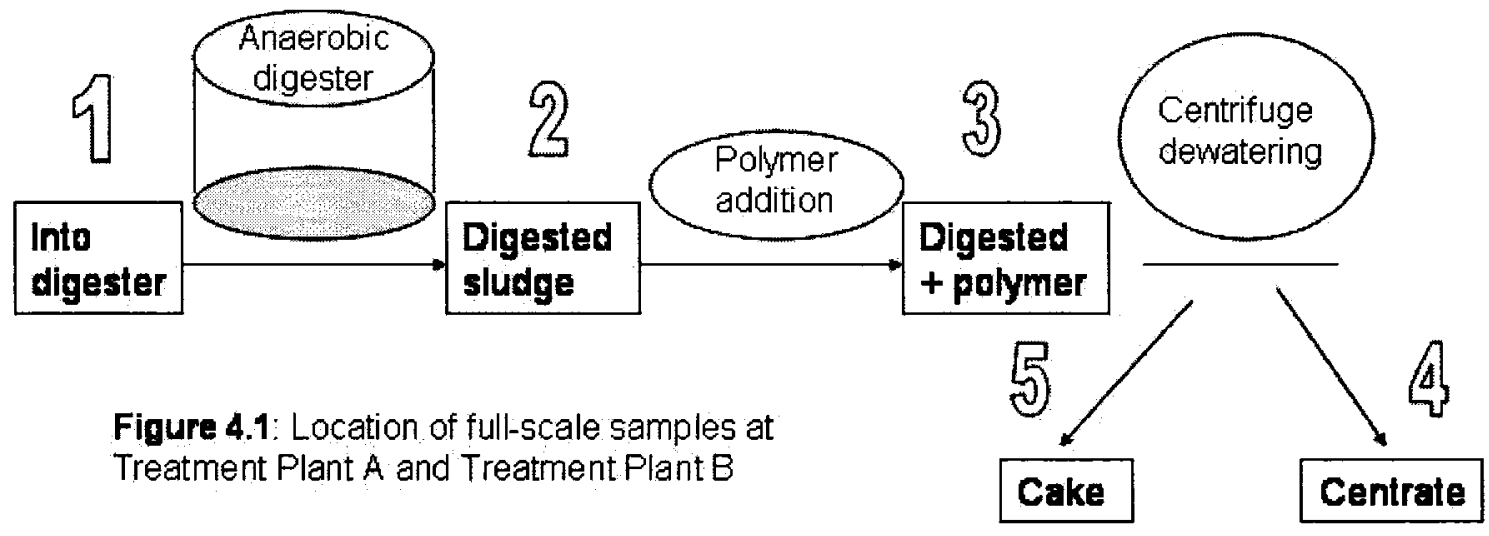

\subsubsection{Test 2: Temperature and time effects}

For this test, sample mixtures as laid out in Figure 4.2, were mixed and left to age at three different temperatures: the refrigerator, $4^{\circ} \mathrm{C}\left( \pm 0.5^{\circ} \mathrm{C}\right)$, room temperature, $24^{\circ} \mathrm{C}\left( \pm 1^{\circ} \mathrm{C}\right)$, and in the incubator, $35^{\circ} \mathrm{C}\left( \pm 0.5^{\circ} \mathrm{C}\right)$. They were sampled and enumerated for fecal coliform counts right after the sample preparation as well as after two and four days at these temperatures. The first sample was made up of $200 \mathrm{ml}$ of digested sludge from the treatment plant. The second sample was made up of 20 grams of the dewatered cake obtained either from the treatment plant or from the laboratory and the third sample was $200 \mathrm{ml}$ of centrate obtained from either from the treatment plant itself or from the lab- 
scale centrifuge. For the fourth sample, 20 grams of the cake was placed in a jar with 180 $\mathrm{ml}$ of PBS. This solution was shaken by hand and left to settle. The fifth sample was made up of 20 grams of cake and $180 \mathrm{ml}$ of centrate obtained from either the treatment plant of the laboratory centrifuge. They were either: placed in the refrigerator, at $4^{\circ} \mathrm{C}$ $\left( \pm 0.5^{\circ} \mathrm{C}\right)$, left out at room temperature, $24^{\circ} \mathrm{C}\left( \pm 1^{\circ} \mathrm{C}\right)$, or put in an incubator, at $35^{\circ} \mathrm{C}$ $\left( \pm 0.5^{\circ} \mathrm{C}\right)$. Cake, centrate and digested sludge samples were obtained from both treatment plants and bacterial enumerations were conducted immediately following sampling as well as after two days and four days. In addition, for a few samples, the enumeration was undertaken after seven days and 14 days at these temperatures.
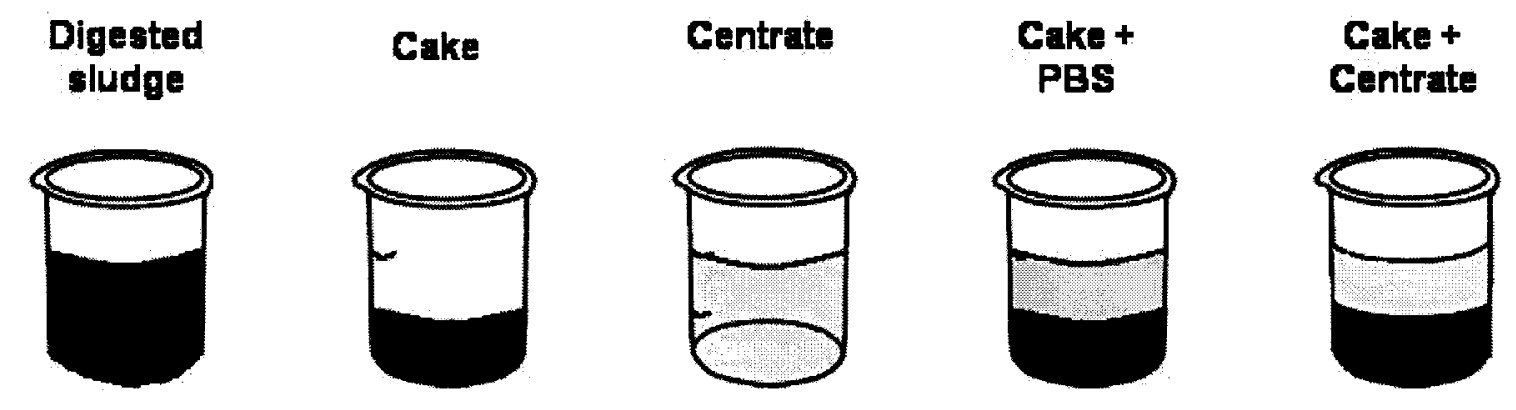

Figure 4.2: Sample types for Test 2, aged for 2 and 4 days at $4^{\circ} \mathrm{C}, 24^{\circ} \mathrm{C}$ and $35^{\circ} \mathrm{C}$

\subsubsection{Test 3: Centrate characterisation}

Chemical characterisation of the dewatering centrate collected from Treatment Plant A was compared with centrate obtained by laboratory centrifugation of digested sludge from the same treatment plant. The parameters examined are: Protein, Carbohydrates, Total Organic Carbon (TOC), Chemical Oxygen Demand (COD), total nitrogen, nitrate, nitrite and ammonia as well as reactive phosphate, sulphide, volatile acids, $\mathrm{pH}$ and the 
solids concentration. Depending on their concentration, these species could either promote or inhibit the growth of fecal coliforms. Detailed methods for each of the parameters can be found in Section 4.2.4.

\subsubsection{Test 4: Centrate seeding}

The goal of this test was to compare the toxicity of the two different types of centrate. This could be observed by the growth and decline in the population of a seeded bacterial culture. Centrate was collected from Treatment Plant A and compared with samples obtained from laboratory centrifuging of digested sludge from Treatment Plant A. Both samples were sterilised prior to their spiking with known bacterial cultures and the sterilisation was verified with non-selective agar (Section 4.2.5).

Two separate runs were undertaken. The first run used a total coliform colony from digested sludge which had been isolated by membrane filtration. These bacteria were isolated from the digested sludge and were good surrogates to the indigenous bacteria in the centrate. The second run used a purchased bacterial strain ( $E$ coli ATTC 11229) to spike the sterilised centrate. The growth and decline in these centrate samples was compared with a spiked phosphate buffer solution (PBS) sample. The spiked PBS sample would behave like a nutrient deprived environment and it could thus compare the effect of nutrients to the samples. Immediately after seeding, the samples were placed at $4^{\circ} \mathrm{C}$ $\left( \pm 0.5^{\circ} \mathrm{C}\right), 24^{\circ} \mathrm{C}\left( \pm 1^{\circ} \mathrm{C}\right)$ and $35^{\circ} \mathrm{C}\left( \pm 0.5^{\circ} \mathrm{C}\right)$ and bacterial enumeration was conducted right after seeding as well as after one day, two days and four days. 


\subsubsection{Test 5: Cellular ATP}

Measuring the intracellular and the extracellular ATP concentrations within a wastewater sample can be used to compare their toxicity as well as giving us a measure of their total cell count. When bacteria are in a stressed environment, cell lysis will occur and the intracellular ATP concentrations will decrease and the extracellular ATP will increase. Therefore the ratio of intracellular to extracellular ATP gives an idea of the biological stress index (BSI \%). Also, a cell which is viable will contain intracellular ATP. A sample with more intracellular ATP has a higher amount of viable cells. In this case, the intracellular ATP concentrations were converted into $\mu \mathrm{g} \mathrm{ATP/g} \mathrm{DW} \mathrm{by} \mathrm{dividing} \mathrm{by} \mathrm{the}$ solids concentration of the sample. Some samples did not require conversion as they were only compared with samples of the same or similar solid concentrations. More information on the method used for the cellular ATP concentrations can be found in Section 4.2.7.

Samples taken at the locations outlined in Figure 4.1 were also studied for the cellular ATP concentrations to compare with the culturable bacteria data obtained from the fullscale. Also, samples of cake, lab-scale and full-scale centrate were placed at $4^{\circ} \mathrm{C}$ $\left( \pm 0.5^{\circ} \mathrm{C}\right), 24^{\circ} \mathrm{C}\left( \pm 1^{\circ} \mathrm{C}\right)$ and $35^{\circ} \mathrm{C}\left( \pm 0.5^{\circ} \mathrm{C}\right)$ and bacterial enumeration was undertaken using both the MPN method and the cellular ATP method. Sampling was conducted on subsequent days for 30 days $(2,4,8,15,21$ and 29 days after incubation) in order to see the effect of the time and temperature on the levels of ATP and culturable bacteria. 


\subsubsection{Treatment plant sampling}

Since this phase of the project had so many different tests associated with it, the sampling varied greatly from week to week. The first test used samples from both Treatment Plant A and Treatment Plant B, whereas for the remainder of the tests, only Treatment Plant A samples were used. Laboratory generated centrate obtained from Treatment Plant A digested sludge was compared with the full-scale centrate samples. Samples of anaerobically digested sludge were collected in the same way as Phase I (Section 4.1.3).

Full-scale samples were obtained from the same treatment plants outlined in Table 4.1 and Table 1.2 and brought to Carleton University for same day analysis. They were analysed within four hours of collection and kept at $4^{\circ} \mathrm{C}\left( \pm 0.5^{\circ} \mathrm{C}\right)$ until the time came for the bacterial enumeration. Site three in Figure 4.1 from Section 4.2.1.1 is the digested sludge mixed with the polymer, immediately prior to the centrifuge dewatering. It was not possible to obtain the sludge samples already mixed with the polymer for either treatment plant. For this reason, already mixed polymer from the treatment plant was added to the digested sludge in the laboratory to give the desired dose of the treatment plant. Polymer was added to a $200 \mathrm{ml}$ sample of digested sludge and mixed using a jar test apparatus at 300rpm for 2 minutes. Treatment Plant A dosed their polymer at $11 \mathrm{~g} / \mathrm{kg}$ DS and Treatment Plant B dosed theirs at $8.4 \mathrm{~g} / \mathrm{kg}$ DS.

Sampling site one of Figure 4.1 is the sludge which enters the anaerobic digester. For

Treatment Plant B, thickened waste activated sludge and primary sludge enters by the 
same feed and could be sampled as one, it had an average solids concentration of $2.5 \%$. For treatment Plant A, however, separate feeds of thickened waste activated sludge and primary sludge entered into the digester with ratios that depended on the precipitation and the season and their subsequent effect on the levels of the different sludges in the treatment plant. The ratio was typically between $66-75 \%$ raw sludge entering into the digester during the months of these tests (summer and fall months). The Cellular ATP test was conducted in the spring, in this case the ratio was $66 \%$ thickened waste activated sludge and $33 \%$ raw sludge.

Polyacrylamide powder polymers were obtained from the respective treatment plants that use them for the conditioning of their sludge. Already mixed polymer solutions were used for the full-scale samples. Treatment plant A used tap water to make up their polymer solution and used a working dose of $0.2 \%$. Treatment plant B used secondary effluent as their make-up water and a working dose of $0.19 \%$.

\subsubsection{Lab-scale centrate sampling}

For lab-scale centrate sampling, anaerobically digested sludge samples were obtained as outlined in Section 4.1.3 and were taken to Carleton University where they were dispensed into $250 \mathrm{ml}$ centrifuge jars and run through a refrigerated Sorval $\mathrm{RC}-5 \mathrm{~B}$ centrifuge for 5 minutes, excluding acceleration and deceleration time at $10,000 \mathrm{rpm}$ $(15,180 \times \mathrm{g})$. The centrate was immediately separated from the sludge cake in order to minimise the resuspension of the sludge cake into the supernatant. Lab-scale centrate was kept at $4^{\circ} \mathrm{C}\left( \pm 0.5^{\circ} \mathrm{C}\right)$ until it was used which was always within 4 hours of centrifugation. 


\subsubsection{Specific methodology for centrate parameters}

A Hach DR 2800 spectrophotometer (HACH, Colorado) was used to measure the levels of the following species: chemical oxygen demand, total nitrogen, nitrate, nitrite, ammonia, reactive phosphate, sulphide and volatile acids as well as total organic carbon for the first sampling event. The protein and carbohydrate concentrations were measured according to the Coomassie and Anthrone methods respectively and the total organic carbon was measured using a TOC- $\mathrm{V}_{\mathrm{CPN}}$ analyzer (Mandel, Guelph, ON) for the second run of samples.

\subsubsection{HACH chemical analyses}

The Beer-Lambert law equates the linear relationship between the absorbance and the concentration of a certain species. This law can help to determine the concentration of parameters within water samples by using a spectrophotometer to detect changes in the sample colour at a certain wavelength. In the case of this project, a HACH DR 2800 spectrophotometer was used with many parameters being tested using established protocols and prepared vials as described in the following sections. This eliminated the need for the generation of colour response curves. Nine different parameters were tested using the prepared reagents which were all purchased from Anachemia Science (Lachine, QC).

\section{1) Chemical Oxygen demand}

The method for the COD analysis is outlined in: HACH Company, (2005b). In order to determine the concentration, the sample needed to be digested; this digestion lasted two 
hours at $150^{\circ} \mathrm{C} . \mathrm{A} \mathrm{DRB} 200$ reactor was used in order to heat the samples. Because the samples of centrate had high COD values, $0.2 \mathrm{ml}$ of sample was added to the digestion reagent vials and the value given in $\mathrm{mg} / \mathrm{L}$ was multiplied by a factor of ten. To summarise the procedure, the vials contain a solution with a dichromate ion $\left(\mathrm{Cr}_{2} \mathrm{O}_{7}\right)$. During the two hour digestion period, the oxidisable organic compounds in the sample react with the strong oxidising agent, potassium dichromate, and the dichromate ion is reduced to a chromic ion $\left(\mathrm{Cr}^{3+}\right)$, this ion is green in colour and measuring the absorbance at a wavelength of $620 \mathrm{~nm}$ can determine the concentration of the species.

\section{2) Total Nitrogen}

The detailed methodology for the total nitrogen concentration is outlined in: $\mathrm{HACH}$ Company, (2005g). For this method, the sample needed to be digested and a DRB200 reactor was used to heat the vials at $105^{\circ} \mathrm{C}$ for 30 minutes. The samples had a high concentration of total Nitrogen and they thus needed to be diluted 10 fold. The concentration values obtained were then multiplied by 10 to get the real value. Values were given in $\mathrm{mg} / \mathrm{L}$ of Nitrogen. In this method the digestion with persulfate converts all forms of nitrogen into nitrate and this nitrate reacts with a strong acid to form a yellow complex whose absorbance is measured at $410 \mathrm{~nm}$.

\section{3) Nitrate}

The nitrate methodology can be found in: HACH Company, (2005c). Values were given in $\mathrm{mg} / \mathrm{L}$ of $\mathrm{NO}_{3}$ - Nitrogen. No dilution was necessary for the sample as the values fell 
within the range of the test. For the reaction, the nitrate within the sample reacts with a strong acid to form a yellow complex whose absorbance is measured at $410 \mathrm{~nm}$.

\section{4) Ammonia}

The methodology for the ammonia nitrogen is outlined in: HACH Company, (2005a). Since levels of Ammonia in the centrate are high, the samples needed to be diluted 100 fold, the range of detection then being $40-5000 \mathrm{mg} / \mathrm{L}$. The value was given in terms of $\mathrm{mg} / \mathrm{L}$ of $\mathrm{NH}_{3}-$ Nitrogen and was multiplied by 100 in order to get the true value. Ammonia in the sample reacts with chlorine to form monochloramine, this monochloramine then reacts with salicylate to form a substance called 5-aminosalicylate, oxidation of this species yields a blue colour. The excess reagent in the sample forms a yellow colour, together with the blue colour yielded by the above reaction; a greencoloured solution is formed and read at $655 \mathrm{~nm}$.

\section{5) Nitrite}

For this method, Test N Tube Plus vials were purchased which meant that no blanks were needed and the test took only ten minutes, methodology can be found in: $\mathrm{HACH}$ Company, (2005d). As this was a test for low concentrations, the sample was diluted tenfold. The values were given in terms of $\mathrm{mg} / \mathrm{L}$ of $\mathrm{NO}_{2}-$ Nitrogen and were multiplied by a factor of ten in order to get the true value. Nitrite in the sample reacts with a primary aromatic amine in an acidic solution, this forms a complex whose colour is proportional to the amount of nitrite present and can be detected at $515 \mathrm{~nm}$. 


\section{6) Reactive Phosphate}

The concentration of orthophosphate was detected using a modified method of 4500-P C from Standard Methods for the Examination of Water and Wastewater (American Public Health Association (APHA), American Water Works Association and Water Pollution Control Federation, 1989). Details of the method can be found in: HACH Company, (2005e). As the concentrations were high, samples still needed to be diluted tenfold in order to detect the right range; values were given in terms of $\mathrm{mg} / \mathrm{L}$ of $\mathrm{PO}_{4}^{-3}$ and were multiplied by ten to get the actual value. In this method, molybdate in an acid solution reacts with the orthophosphate in the sample to form an acid which in the presence of vanadium yields a yellow colour which is proportional to the concentration of reactive phosphate and can be read at $420 \mathrm{~nm}$.

\section{7) Sulphide}

The Methylene Blue Method for the detection of Sulphide in Water and Wastewater was adapted from Protocol 4500-S- $\mathrm{D}$ in: (American Public Health Association (APHA), American Water Works Association and Water Pollution Control Federation, 1989). The sulphide method undertaken for the study is detailed in: HACH Company, (2005f). Since there was no higher range for sulphide detection and because the sulphide concentrations were higher than the range of the test, the samples needed to be diluted tenfold, values given in $\mathrm{mg} / \mathrm{L}$ of $\mathrm{S}^{2-}$ were then multiplied by ten in order to get the real value. In the reaction, the hydrogen sulphide and the acid-soluble sulphide reacts with dimethyl- 
henylenediamine sulphate to form methylene blue, the more intense the blue colour the higher the concentration of sulphides, absorbance is read at $665 \mathrm{~nm}$.

\section{8) Volatile acids}

The method used for this project was specific for digester sludges but it was possible to use it for our samples with very little modification. Detailed methodology can be found in: HACH Company, (2005i). Samples needed to be filtered prior to determination as centrifuging was not sufficient for solids separation. Sample cells needed to be boiled in a water bath. For this, the sample cells were placed in a $500 \mathrm{ml}$ beaker with boiling water for three minutes. Values are given in terms of $\mathrm{mg} / \mathrm{L}$ of acetic acid (HOAC). The volatile acids in the sample are determined by esterification of the carboxylic acids present in the samples, the esters formed are determined by measurement of the absorbance of the sample at $495 \mathrm{~nm}$.

\section{9) Total Organic Carbon}

Measurements for total organic carbon were also undertaken by using a TOC Analyser, methodology for this is found in Section 4.2.4.6. Detailed methodology for the direct method using the spectrophotometer can be found in: HACH Company, (2005h). For this method, the samples needed to be digested and a DRB200 reactor was used to keep samples at $105^{\circ} \mathrm{C}$ for two hours. Samples had high level of TOC and thus needed to be diluted tenfold, values were given in terms of $\mathrm{mg} / \mathrm{L}$ of Carbon and multiplied by ten to obtain the actual value. In summary, the organic carbon is determined by first eliminating 
any of the inorganic carbon from the sample by putting it in slightly acidic conditions. Organic carbon is then converted to carbon dioxide in the presence of persulphate. $\mathrm{A} \mathrm{pH}$ indicator reagent vial is inserted into the sample and the carbon dioxide in the vial forms carbonic acid and changes the $\mathrm{pH}$ and thus changes the colour of the solution. Absorbance of samples is measured at 598 and 430nm.

\subsubsection{Protein concentration}

The protein concentrations were determined by using a Coomassie Brilliant Blue G-250 reagent with the Bradford method (Bradford, 1976). This method was chosen because it had a very high sensitivity and very few interfering substances. The main interfering substances are strongly alkaline buffering agents as well as certain detergents, neither of which was expected in significant quantities in the centrate samples. The Coomassie (Bradford) reagent kit was purchased from Pierce Biotechnology Inc (Rockford, IL) which came with protein assay kit already prepared (Pierce, 2004). It contained Bovine Serum Albumin (BSA) at a concentrated solution of $2 \mathrm{mg} / \mathrm{ml}$ which could be diluted in order to produce a colour response curve. Preliminary testing showed that both types of centrate had protein concentrations in the working range of 100-150 $\mathrm{mg} / \mathrm{L}$ and for this reason, the standard test tube protocol was used. The following is the standard protocol with its modifications undertaken for the project:

1. Fill test tubes with $5 \mathrm{ml}$ of the Coomassie Reagent

2. Pipette in $0.1 \mathrm{ml}$ of each standard or unknown sample and mix well

3. Incubate samples at room temperature for ten minutes 
4. Set the HACH spectrophotometer DR2800 to $595 \mathrm{~nm}$ and zero the instrument with a test tube filled only with distilled water.

5. Subtract the average $595 \mathrm{~nm}$ measurement of the blank sample, made up of $5 \mathrm{ml}$ of Commassie Reagent and $0.1 \mathrm{ml}$ of distilled water, from the measurements of all other individual samples.

The preparation of the standard curve included both low levels and high levels of protein within the centrate samples. At first, both low-range and high-range concentrations (up to $2000 \mathrm{mg} / \mathrm{L}$ ) were prepared and centrate samples were tested in order to see the working range. As average concentrations were approximately $200 \mathrm{mg} / \mathrm{L}$, the colour response curve was done using the following BSA concentrations: $0,25,50,75,100,125,150$, $175,200,250,300$ and $350 \mathrm{mg} / \mathrm{L}$. The dilutions were duplicated and as the curves obtained by each replicate had the same slope $(0.0018)$ it was possible to combine the two replicates together to make one curve. The colour response curve is given as Figure 4.3 with the $r$ squared value and the linear equation on the figure as well. All concentrations of protein were done by using this equation. 


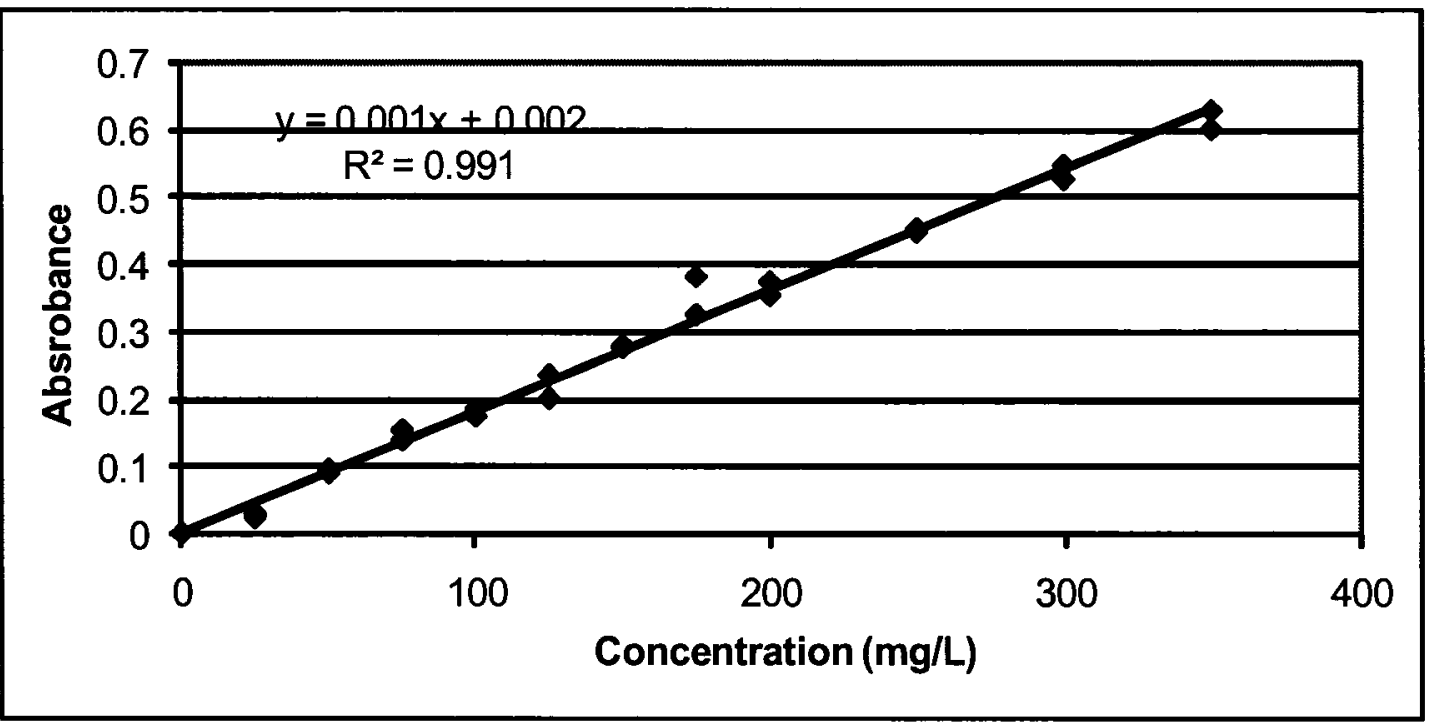

Figure 4.3: Colour response curve for BSA between 0-350 $\mathrm{mg} / \mathrm{L}$

\subsubsection{Carbohydrate concentration}

Carbohydrate concentrations were found using the Anthrone method. Morris (1948) was the first to present this method using Dreywood's Anthrone Reagent (Morris, 1948). There are many different ways described in the literature for the make-up of the solution but most use a strong concentrated acid to dissolve the Anthrone reagent obtained from: Acros Organics, NJ, and have a mixture of one part test sample for two parts Anthrone solution (Koehler, 1952). The following is a summary of the protocol used for this project:

1. Prepare a $95 \%$ sulphuric acid concentration by adding $950 \mathrm{ml}$ of concentrated sulphuric acid to $50 \mathrm{ml}$ of water, alternatively, a prepared $95 \%$ sulphuric acid solution can be used 
2. Dissolve 2 grams of Anthrone reagent for each litre of sulphuric acid solution

3. Fill test tubes with $6 \mathrm{ml}$ of the Anthrone solution

4. Pipette in $3 \mathrm{ml}$ of each standard or unknown sample and mix well

5. Incubate samples at room temperature for ten minutes

6. Set the HACH spectrophotometer DR2800 to $620 \mathrm{~nm}$ and zero the instrument with a test tube filled with distilled water.

7. Subtract the average $620 \mathrm{~nm}$ measurement of the blank sample, made up of $6 \mathrm{ml}$ of Anthrone solution and $3 \mathrm{ml}$ of distilled water, from the measurements of all other individual samples.

Preparation of a standard curve was done that would include both low levels and high levels of protein within the centrate samples. Preliminary tests showed that the centrate samples both had concentrations of carbohydrates of approximately $150 \mathrm{mg} / \mathrm{L}$ and a working range from 0 to $200 \mathrm{mg} / \mathrm{L}$ was used. The colour response curve was done using the following galactose (Fisher Scientific, Toronto, ON) concentrations: $0,20,40,60,80$, $100,120,140,160,180$ and $200 \mathrm{mg} / \mathrm{L}$. These concentrations were done in duplicate and as the curves obtained by each replicate had the same slope $(0.009)$ it was possible to combine the two replicates onto the same curve. The colour response curve is given as Figure 4.4 with the $r$ squared value and the linear equation on the figure as well. All concentrations of carbohydrates were done by using this equation. 


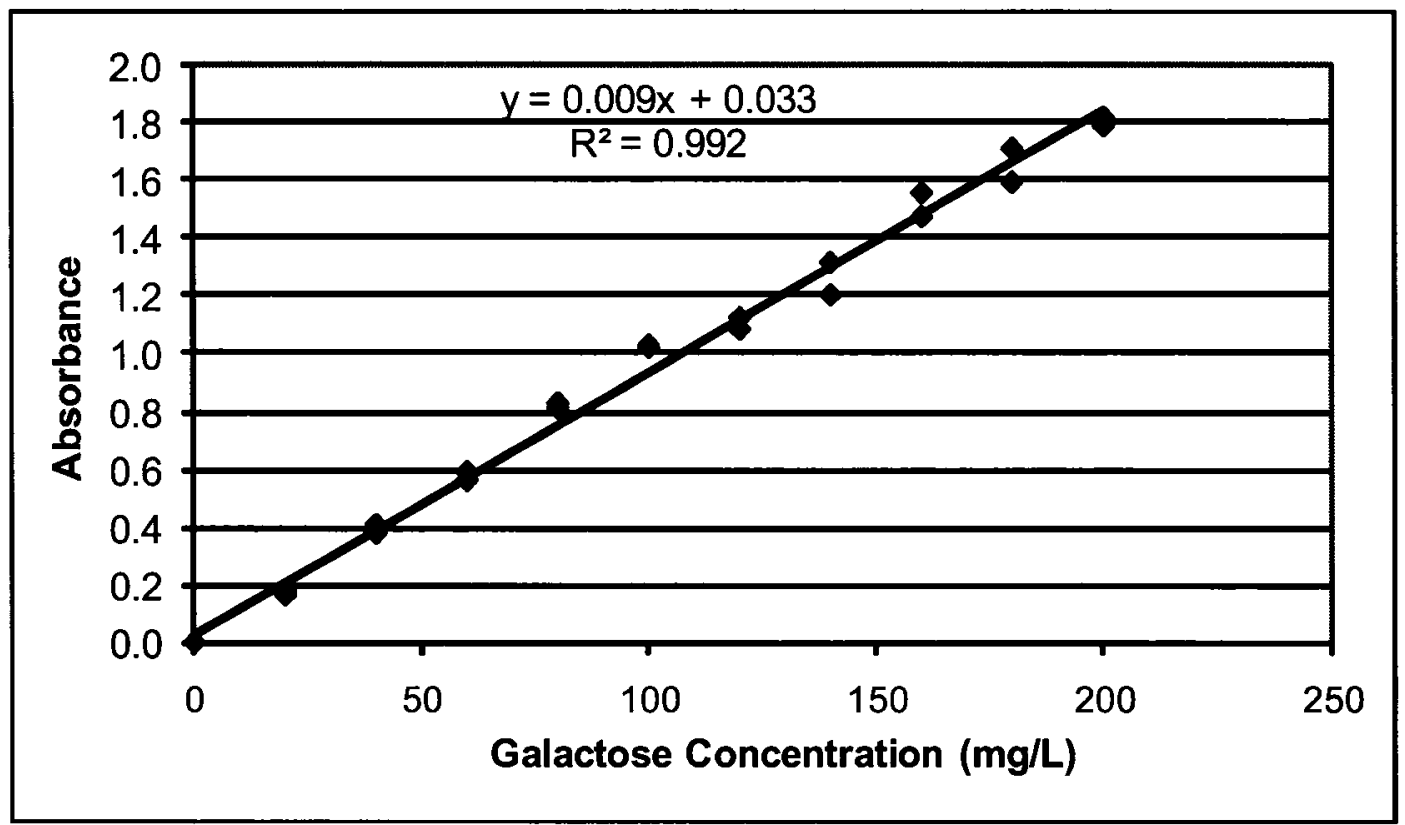

Figure 4.4: Colour response curve for galactose between 0 and $200 \mathrm{mg} / \mathrm{L}$

\subsubsection{4. $\mathrm{pH}$}

The $\mathrm{pH}$ of the solution was determined by using a handheld portable $\mathrm{pH}$ meter (SympHony SP70P by VWR, Missassauga, ON). The $\mathrm{pH}$ meter was calibrated before the first sampling of the day. Samples of centrate were placed in $50 \mathrm{ml}$ beakers and the electrode was rinsed thoroughly with distilled water before and after each sample and replicate.

\subsubsection{Total solids}

The total solids for each of the samples were found in the same way as those that were used to convert the MPN values / $\mathrm{g}$ DW, as described in Protocol $2540 \mathrm{~B}$ in (American Public Health Association (APHA), American Water Works Association and Water Pollution Control Federation, 1989). Dry crucibles were weighed and then filled with 15 
to $20 \mathrm{ml}$ of the centrate samples and reweighed before being placed in an oven at $105^{\circ} \mathrm{c}$ overnight. Samples were then placed in a dessicator before they were weighed. Total solids: $\left(\mathrm{W}_{\text {dry }}-\mathrm{W}_{\text {crucible }}\right) /\left(\mathrm{W}_{\text {sample }}-\mathrm{W}_{\text {crucible }}\right) \times 100 \%$.

\subsubsection{Total Organic Carbon}

Another way for the Total Organic Carbon to be determined was by using a TOC-V $\mathrm{V}_{\mathrm{CPN}}$ analyzer by Shimadzu. The general method used for this apparatus can be found in Protocol 5310B in (American Public Health Association (APHA), American Water Works Association and Water Pollution Control Federation, 1989). Samples of centrate needed to be filtered and diluted tenfold in order to be able to be placed in the ASI-V automatic sampler for water samples. Without qualitative filtration, the larger particles would have interfered with the sampling technique. A dose response curve was obtained using the TOC-Control V software included in the analyzer, it is shown in Figure 4.5. This graph equates the area under the curve with the concentration of total organic carbon. 


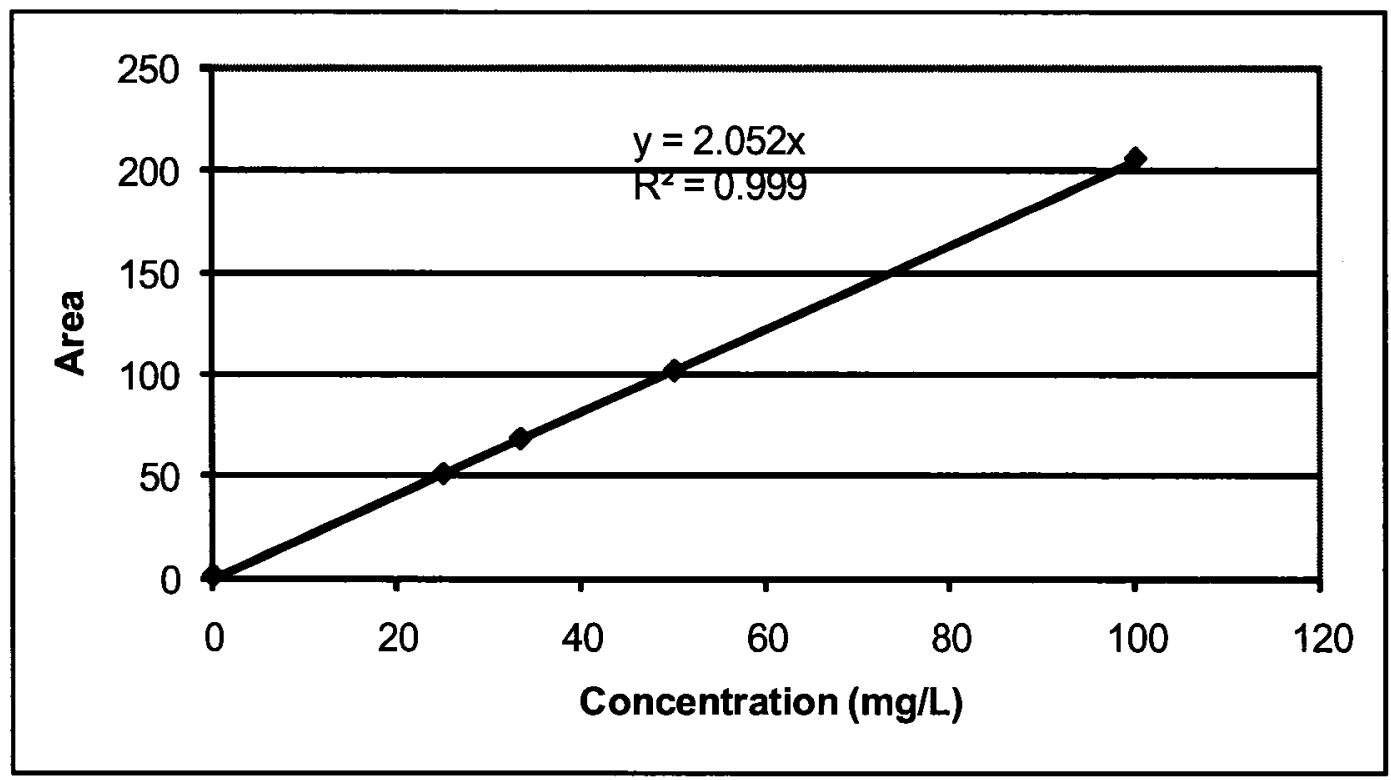

Figure 4.5: Calibration curve for Total Organic Carbon from TOC- $\mathrm{V}_{\mathrm{CPN}}$

\subsubsection{Sterilisation of centrate}

Samples of both full-scale and lab-scale centrate were sterilised for Test 4 , the centrate seeding test. It was decided that filter sterilisation would be ideal for the centrate, however, it was extremely time-consuming and the samples would then not include any of the larger species that would have been filtered out.

The sterilisation was achieved by ultraviolet light (UV). Samples were first filtered using qualitative pore-sized filters in order to remove the larger particles which could shield the pathogens during UV sterilisation. $50 \mathrm{ml}$ samples were placed in a petri dish with a $6 \mathrm{~cm}$ diameter, the depth of the water being $1.77 \mathrm{~cm}$. The sample was continuously mixed during the four minutes of sterilisation with a magnetic stirrer. 4 UV-C lamps of 15 Watts at a distance of $46 \mathrm{~cm}$ from the sample sterilised the solution. Samples were tested for their sterility by spreading on non-selective media, R2A Agar (Fisher Scientific, Toronto, 
ON) and incubated in a covered petri dish for up to 4 days at $35^{\circ} \mathrm{C}$; none of the samples showed any growth on the agar plates during this time.

\subsubsection{Methodology for fecal coliform seeding}

Two separate methods were undertaken for the seeding of sterilised centrate samples. The first one was used in order to isolate indigenous bacteria from a sludge sample. In order to do this, a digested sludge sample was membrane filtered using a $0.45 \mu \mathrm{m}$ pore size filter placed onto LES Endo agar: Protocol 9222 B (American Public Health Association (APHA), American Water Works Association and Water Pollution Control Federation, 1989). After incubation for 24 hours at $35^{\circ} \mathrm{C}$, the agar plate was taken out of the refrigerator and three colony forming units were carefully removed using a sterilised inoculating loop and dispensed into $200 \mathrm{ml}$ of Tryptic Soy Broth. This solution was then placed into the incubator at $35^{\circ} \mathrm{C}$ between three hours and 24 hours before seeding occurred. $15 \mathrm{ml}$ of the broth was added to $350 \mathrm{ml}$ sterilised centrate solution. The fecal coliform quantification procedure using the A-1 Media was immediately undertaken and samples were placed at $24^{\circ} \mathrm{C} \pm 1{ }^{\circ} \mathrm{C}$ and $35^{\circ} \mathrm{C} \pm 0.5^{\circ} \mathrm{C}$ and bacterial enumeration was done at days one, two and four.

For the second fecal coliform seeding test a single strain of a microorganism was used so that no competition from other bacteria in the sample would interfere with the survival of the microorganism. A strain of $E$ coli (ATCC \#11229) was purchased from Med-ox Diagnostics (Ottawa, ON). The bacteria came in the form of Kwik Stik devices which had lyophised pellets of the $E$ coli strain in question (MicroBiologics, 2004). After the 
hydrating fluid was added to the pellet, the strain was spread onto an R2A agar plate in a circular pattern; the inoculated area was then streaked with an inoculating loop in order to facilitate colony isolation. The primary culture plates were then placed in the incubator at $35^{\circ} \mathrm{C}$ for 24 hours. After this time, an inoculating loop was used to remove three colonyforming units and placing them in $350 \mathrm{ml}$ samples of UV sterilised centrate samples or in sterilised PBS dilution water. Bacterial enumeration was undertaken immediately following inoculation of the centrate and then samples were placed at $4^{\circ} \mathrm{C} \pm 0.5^{\circ} \mathrm{C}, 24^{\circ} \mathrm{C}$ $\pm 1^{\circ} \mathrm{C}$ and $35^{\circ} \mathrm{C} \pm 0.5^{\circ} \mathrm{C}$ and sampled again at days one, two and four.

\subsubsection{Cellular ATP methodology}

The goals of the cellular ATP test were two-fold; firstly it was used to gauge the relative toxicity of certain samples. Secondly, the relative amount of viable cells could be quantified by measuring the amount of intracellular ATP in the samples. The methodology for these tests was quite simple; the entire test took under 20 minutes. A Lumitester C-100 (Kikkoman Corporation, LuminUltra, Fredericton, NB) was obtained. The dissolved and total ATP concentrations were calculated from: LuminUltra Technologies, (2007). The results were given in Relative Light Units and for each run of samples calibration was required by measuring the RLU obtained for a sample of UltraCheck and using that to calibrate the other samples.

The methodology from LuminUltra was developed for use with biological wastewater treatment effluent which has low solids concentrations, modifications had to be done in order to use this method for cake samples with solids concentration in the $30 \%$ range. 
Upon discussion with LuminUltra Technologies it was decided that the following changes would be ideal for the cake samples (J. Cairns, 2007, personal communication). For the total ATP calculations, instead of pipetting $1 \mathrm{ml}$ of the sample into the test tubes, $1 \mathrm{~g}$ of the cake was weighed out and placed in the test tubes and mixed by hand. This made the test possible but a degree of error may have occurred because the cake was found in a large solid mass making it difficult for the reaction to take place as the reagent could only react with the cake around the mass and not within it.

For the extracellular ATP calculations, only $0.1 \mathrm{ml}$ of sample was used, error produced in weighing out such a small sample was deemed to be too high and the method was modified further so that $1 \mathrm{~g}$ was weighed out added to a test tube with the LumiSolve reagent in it and mixed vigorously and then immediately after, $1 \mathrm{ml}$ of that solution was pipetted into another test tube with a further $10 \mathrm{ml}$ of LumiSolve reagent in it. For this sampling method as well, the same error as the total ATP may have been present, where it could be possible that the reagent could not reach the cake within the solid mass which could lead to lower than expected numbers for the extracellular and total ATP from the Lumitester.

The Biomass Stress Index (BSI) was used as a measure of the toxicity of the environment in the samples and is given by the following formula: BSI(\%): extracellular ATP/ total ATP. Both extra and total ATP are given in $\mathrm{ng} / \mathrm{ml}$ or $\mu \mathrm{g} / \mathrm{L}$. A modification of the Active Biomass Ratio (ABR (\%)) was used to gauge the amount of viable biomass. ABR $(\%)=$ (intracellular ATPx0.5) / TSS. Where 0.5 is a conversion factor and TSS is the total 
suspended solids $(\mathrm{mg} / \mathrm{L})$. As the samples for this test had high solids content, it was more logical to divide the samples by their total solids concentration and thus the viable biomass index, a modification of the active biomass ratio, was given as intracellular ATP/ g DW or simply by its level of intracellular ATP in $\mathrm{ng} / \mathrm{ml}$.

\subsubsection{Bacterial enumeration}

Bacterial enumeration was performed in the same manner as in Phase I of the experiment (Section 4.1.6). The A-1 Medium was used for all of the tests in Phase II that required culture methods (US EPA, 2005b).

\subsubsection{Statistical analysis}

Statistical Analysis for Phase II was undertaken in the same fashion as Phase I. SPSS 13 for Windows was used in order to compare the means of the samples by Student t-tests as well as by Analysis of Variance using ANOVA tables and the Least Significant Difference (LSD) post-hoc test. 


\section{Chapter 5}

\section{Results and Discussion}

The results and discussion will be split into the two phases of the project just as the materials and methods were. The first Phase of the project focused on the role that the shear and the addition of polymer could have on the fecal coliform counts. The second phase looked into any changes that could be seen within the treatment plant as well as on the role that the centrate could play in the inhibition of fecal coliform growth.

\subsection{Phase I}

Phase I of the experiment, as was explained in the Materials and Methods section, is composed of two different tests. The first test looked at the effect of centrifuge speed on fecal coliform counts and the second test looked into the effect of polymer addition. For both of these tests, the effect of aging the samples at room temperature was also examined.

\subsubsection{Test 1: Effect of centrifuge speed}

Preliminary tests using the Centra GP8 centrifuge were undertaken in order to gauge the concentrations of the cake that would be expected and the temperature change that could arise through the 5 minutes of centrifugation time. The average solids concentration for the highest centrifuge speed, $4500 \mathrm{rpm}(4140 \mathrm{x} \mathrm{g})$, was $10 \%$. By augmenting the centrifugation time to 9 minutes, it was possible to obtain a solid concentration of $11.50 \%$. This increase in solids concentration for a longer centrifugation time was very minimal. In addition to this, the centrifuge which was being used was not refrigerated and 
the temperature rose from $6^{\circ} \mathrm{C}$ to $12^{\circ} \mathrm{C}$ within 5 minutes in the centrifuge. Leaving the samples for a longer time and allowing the temperature to increase could cause undue error. In the case of this project, the samples were never left at higher temperatures, upon exiting the centrifuge they were either enumerated right away or placed in the refrigerator for enumeration within the following two hours.

Even though the centrifuge was leading to lower than expected solids content, three different speeds were selected for Test 1 that represented the same centrifugal force obtained in a full-scale centrifuge. The relative centrifugal forces applied to the samples werer: $1300 \times \mathrm{g}(2500 \mathrm{rpm})$, typical of a conventional or low-solids dewatering centrifuge, $2500 \mathrm{xg}(3500 \mathrm{rpm})$ which would be on the border between a low-solids and a high-solids centrifuge and finally $4140 \times \mathrm{g}(4500 \mathrm{rpm})$ which is in the high range of a high-solids centrifuge.

The first run of samples was enumerated for fecal coliforms using the LTB/ EC method (Method 1680: US EPA, 2005a). Three centrifuge speeds were tested, shown on Figure 5.1 in terms of revs per minute (rpm). Cake and centrate fecal coliform levels were determined for each of the centrifuge speeds as well as after one day of aging. Figure 5.1 has the results from this run of samples. The digested sludge sample had a fecal coliform MPN of $\log 5.1$, as seen on the figure. This figure shows that the levels of fecal coliforms decrease immediately following centrifugation except for the sample of centrate run through the centrifuge at $4500 \mathrm{rpm}(4140 \mathrm{x} \mathrm{g})$ and thus that no immediate postdewatering reactivation occurred. 
The error bars on the figure are those given by the MPN calculator. They depend on the number of samples for which gas is produced after sample incubation. Samples with arrows instead of error bars either had all of their dilutions being positive or negative for fecal coliform growth. The actual fecal coliform culture count is either smaller than or larger than the height of the bar on the graph depending on the orientation of the arrow.

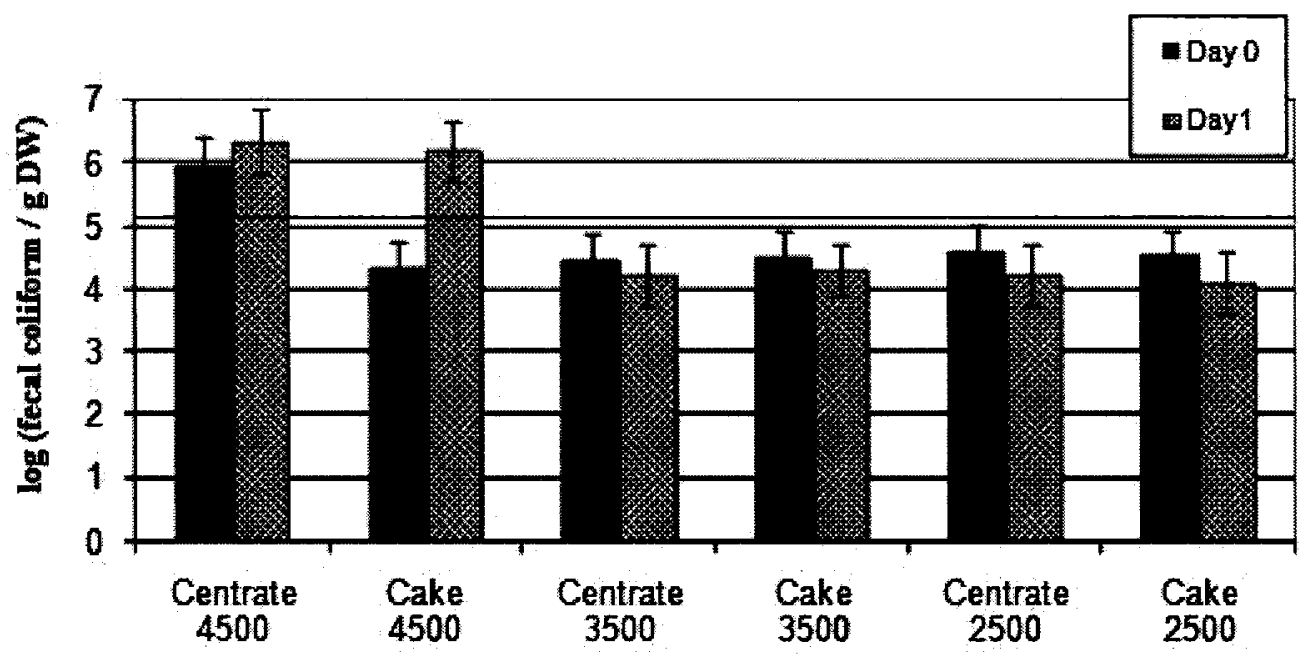

Figure 5.1: Effect of centrifuge speed on fecal coliforms. Detection and enumeration by LTB/ EC medium and aging for 24 hours \pm 3 hours at $24^{\circ} \mathrm{C} \pm 1^{\circ} \mathrm{C}$. Digested sludge from Treatment Plant A, centrifuge speed given in rpm

These tests had centrate and cake values that were able to recover when centrifuged at $4500 \mathrm{rpm}$ but not at the lower speeds. The centrate levels increased by $\log 0.82$ from its digested value immediately following centrifugation and increased a further $\log 0.41$ after 24 hours at room temperature. Centrifugation at $4500 \mathrm{rpm}$ had a significant effect on the fecal coliform counts for the centrate samples $(\mathrm{p}=0.04)$. The cake levels were slightly 
lower than the digested value immediately following centrifugation but after 24 hours at room temperature, the levels of fecal coliforms increased by $1.85 \log$ from the Day 0 value. The effect of aging for 24 hours after centrifugation had a significant effect on the fecal coliform counts, tested using an ANOVA, for cake samples at $4500 \mathrm{rpm}(\mathrm{p}=0.03)$.

The second run of samples was done in the same way as the first, except in this case a one-step detection and enumeration method (US EPA 1681) was undertaken using A-1 Medium. The A-1 methodology is laid out in (US EPA, 2005b). The results for this week are presented in the same fashion as the previous test run, the difference between these two sets being the media used for the enumeration of the fecal coliforms. For these tests, samples were left out at room temperature for 24 hours and 48 hours. Figure 5.2 has the results from the second set of centrifuge speed tests. The digested sludge samples for this sampling event had a fecal coliform count of $\log 4.9$, shown as a line on the figure. Immediately following centrifugation, all samples had lower fecal coliform counts than the digested sludge samples, meaning that no immediate reactivation occurred. As for the aging of the samples, a clear trend could not be observed and no significant effect of aging as well as centrifuge speed was obtained. 


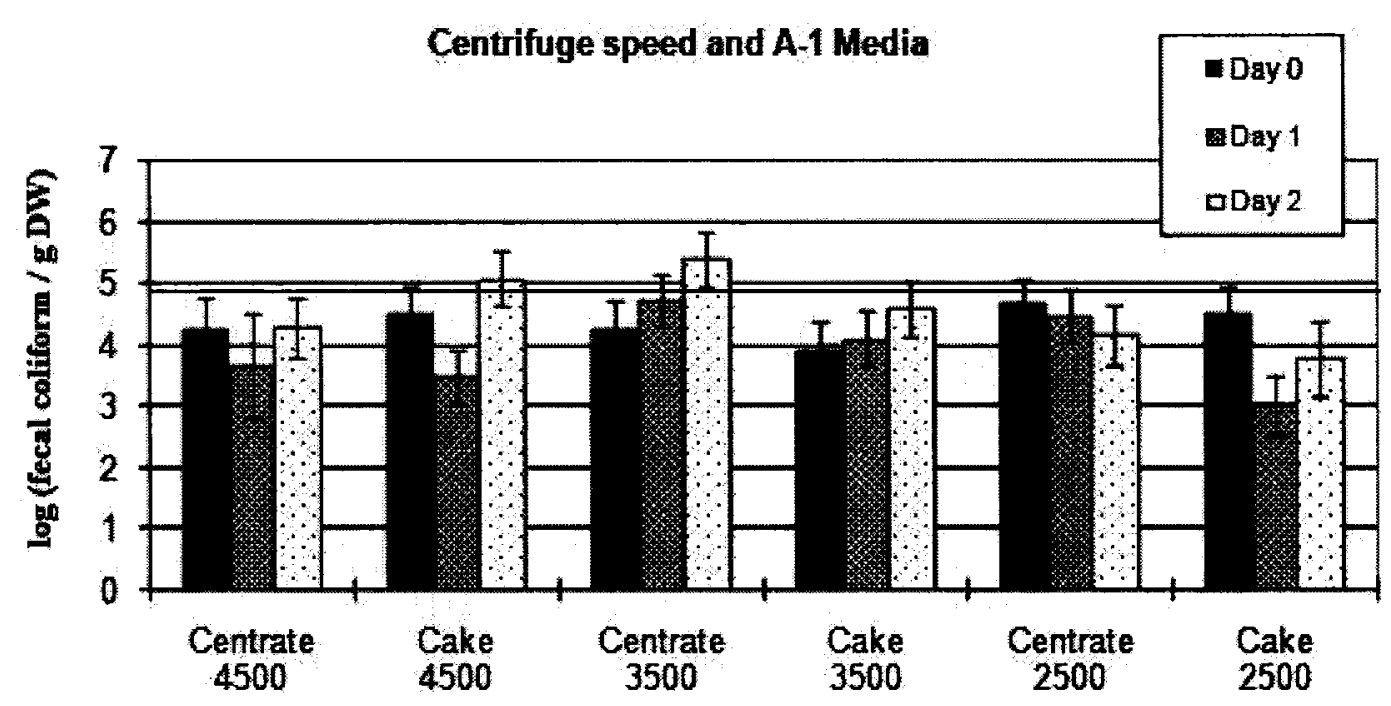

Figure 5.2: Effect of centrifuge speed on fecal coliforms. Detection and enumeration by A-1 medium and aging for 24 hours \pm 3 hours and 48 hours \pm 3 hours at $24^{\circ} \mathrm{C} \pm 1^{\circ} \mathrm{C}$. Digested sludge from Treatment Plant A, centrifuge speed given in rpm

The A-1 method is less time-consuming than the LTB/EC method as the incubation takes only 24 hours and requires less glassware as the detection is done with only one step. However, previous research has reported it having increases in false positives, leading to higher than expected MPN values (Baker et al., 2005). Other research has reported that the use of the A-1 media over LTB/EC media does not significantly affect fecal coliform counts in dewatered sludge samples (Qi et al., 2007). In the case of this study, the results from the two methods were consistent and the choice of enumeration was not seen to have an effect on the fecal coliform count. As the A-1 method was less time-consuming and required less glassware, it was used for all of the subsequent culture count enumerations in the study.

The first hypothesis, which is associated with the Phase I experiments, stated that the shear could cause higher counts when the polymer was stripped off. In order to compare 
the effect of centrifuge shear alone to the effect of shear combined with polymer addition, the next run of samples were mixed with a polymer at its optimum dose prior to centrifugation. CA4600 polymer was used at the optimum dose (15 g/ kg DS), which was found by using torque-rheogram method and the CST method. The results for this sampling run, with the different centrifuge speeds and the aging of samples at room temperature for 24 and 48 hours, are outlined in Figure 5.3. The digested sludge for this sample run had a log fecal coliform MPN of 5.7, as shown by the line on the figure. All of the fecal coliform levels dropped from the level in the digested sludge sample after centrifugation and aging. There are increases within the same samples, such as the centrate run through the centrifuge at $2500 \mathrm{rpm}$ which increases by Day 1 but then decreases to a level which is lower than Day 0 at Day 2. Analyses of Variance (ANOVA) tests were performed on the results of the test to see if either the centrifuge speeds or the aging of the samples had significant effects on the fecal coliform counts. Neither the centrifuge speed nor the aging of the centrate and sludge samples had a significant effect on the fecal coliform counts, $\mathrm{p}=0.47$ for centrifuge speed and centrate samples, $\mathrm{p}=0.89$ for centrifuge speed and sludge cake samples, $p=0.42$ for aging of centrate samples and $\mathrm{p}=0.15$ for aging of sludge cake samples. 


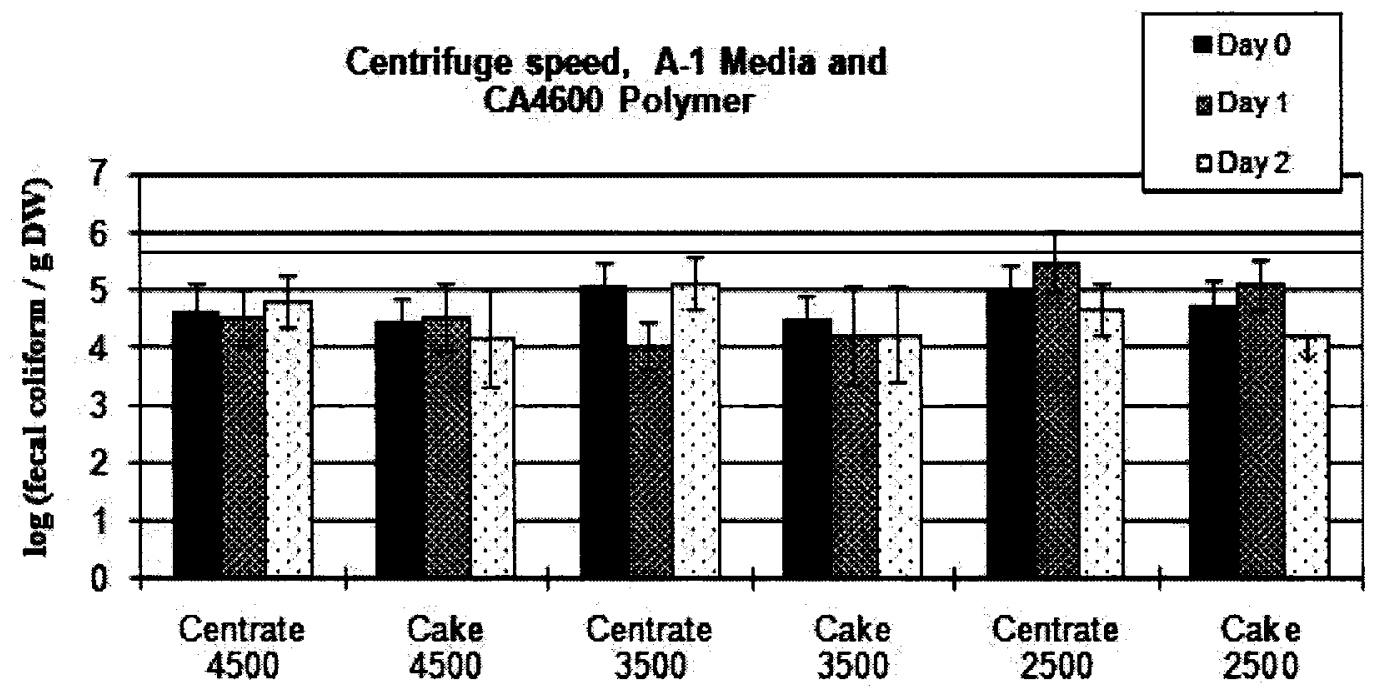

Figure 5.3: Effect of centrifuge speed and polymer addition on fecal coliforms. Detection and enumeration by A- 1 medium and aging for 24 hours \pm 3 hours and 48 hours \pm 3 hours at $24^{\circ} \mathrm{C} \pm 1^{\circ} \mathrm{C}$. Digested sludge from Treatment Plant A, centrifuge speed given in rpm, CA4600 polymer added at optimum dose: $15 \mathrm{~g} / \mathrm{kg}$ DS

It is possible that the addition of polymer can enmesh particles including bacteria and that once put through the centrifuge this polymer can be stripped off releasing the enmeshed particles leading to higher counts on the standard culture methods. With this sampling run, the addition of the polymer at the optimum dose did not lead to an increase in fecal coliforms after centrifugation. As well, the conditions did not allow for the regrowth of the bacteria with aging at the optimum growth temperature of $24^{\circ} \mathrm{C}$.

In the literature, the regrowth/reactivation of fecal coliforms is more pronounced and more common when using a high-solids centrifuge (Qi et al., 2004). It was for this reason that the representative high-solids and low-solids centrifuge speeds were chosen for the centrifuge. Overall, the first test, looking at the effect of centrifuge speed did not show a 
greater increase in fecal coliforms at a higher centrifuge speed than at a lower one. Only one sampling run showed an increase after going through the centrifuge (Figure 5.1) and this increase was seen only after aging of the sample at room temperature. Immediate reactivation was not observed.

The average solids concentrations for the samples run through the Centra GP8 centrifuge at the different speeds are given in Table 5.1. These solids concentrations are for samples without the addition of polymer. At the highest speed, the average cake solids concentration is $10 \%$ and at $2500 \mathrm{rpm}$ the solids concentration was only $5 \%$, this is only a slight increase from the average solids of the digested sludge samples, $2.5 \%$. The centrifuge, at $2500 \mathrm{rpm}$, could not separate the solids from the liquids efficiently. The average solids concentration for the centrate at those speeds is also given in Table 5.1. As expected, when the centrifuge speed decreased, the amount of solids in the centrate increased.

Table 5.1: Average cake and centrate solid concentrations after centrifugation at different speeds using Centra GP8 centrifuge $(n=5)$

\begin{tabular}{|cc|cc|}
\hline $\begin{array}{c}\text { Speed } \\
\text { (rpm) }\end{array}$ & $\begin{array}{c}\text { Relative centrifugal } \\
\text { force (x g) }\end{array}$ & $\begin{array}{c}\text { Average cake } \\
\text { solids (\%) }\end{array}$ & $\begin{array}{c}\text { Average centrate } \\
\text { solids (\%) }\end{array}$ \\
\hline 4500 & 4140 & 10.02 & 0.41 \\
3500 & 2500 & 7.57 & 0.44 \\
2500 & 1300 & 5.46 & 0.50 \\
\hline
\end{tabular}




\subsubsection{Test 2: Addition of polymer dose and type}

All of the results from the second test will be presented in the same way. The figures that follow are given with the different polymer doses on the $\mathrm{x}$-axis and its effect on the MPN values on the y-axis. These figures are split into A and B with the fecal coliform counts in the centrate as a) and the sludge cake samples as b). From the figures, it is possible to examine the effect of centrifuging at different polymer doses as well as the effect of aging the samples at room temperature for 48 hours ( \pm 3 hours). Five different polymer doses were studied, increasing incrementally from a well underdosed sample to a well overdosed sample, the central polymer dose being the optimum dose. Control samples had no polymer added but run through the test the same way as those with polymer added.

For the first run of Test 2 samples, the Centra GP8 centrifuge was used at $4500 \mathrm{rpm}$ for 5 minutes including acceleration and deceleration time. The optimum polymer dose was found to be $15 \mathrm{~g} / \mathrm{kg}$ DS and samples were distributed with polymer doses of 5, 10, 15, 20 and $25 \mathrm{~g} / \mathrm{kg}$ DS. Enumeration was done before and after centrifugation as well as after 2 days of aging. The results are given in Figure 5.4 with a) for centrate samples and b) for sludge cake samples. 
CA 4600 polymer and aging a) Centrate

\begin{tabular}{|l|}
\hline Day 0: No centrifuging \\
ODay 2: No centrifuging \\
Day 0: Centrifuge at $4500 \mathrm{rpm}$ \\
ODay 2: Centrifuge at $4500 \mathrm{rpm}$
\end{tabular}

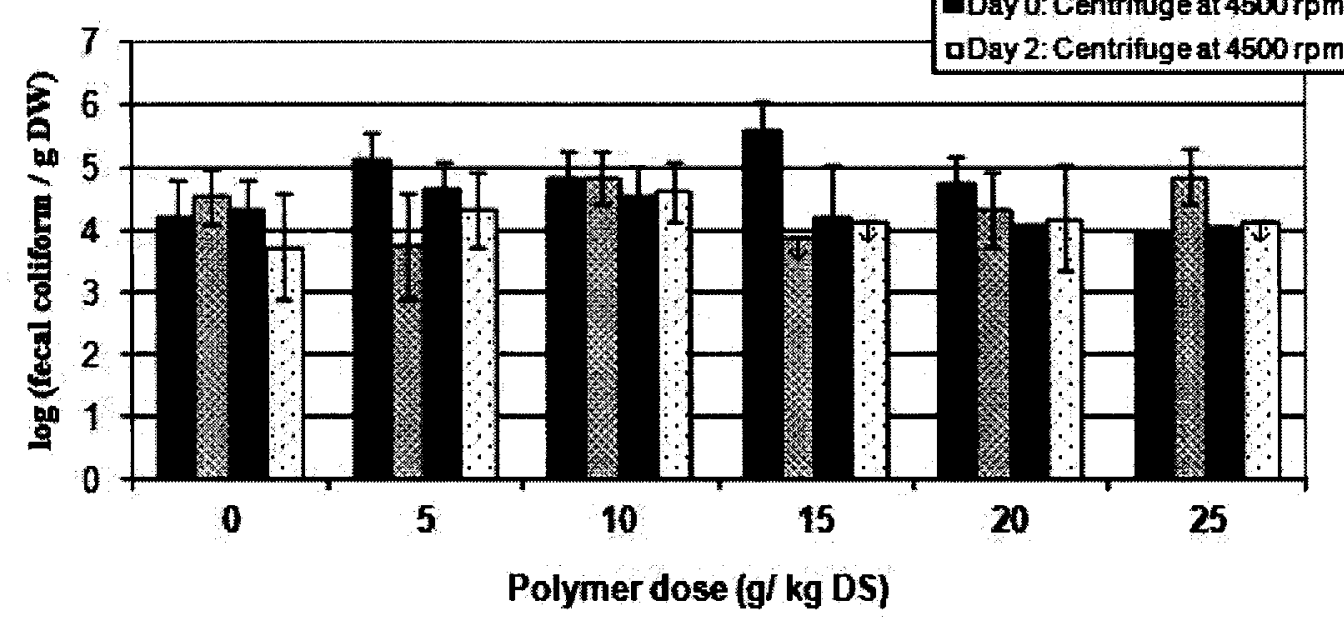

CA 4600 polymer and aging b) Sludge cake

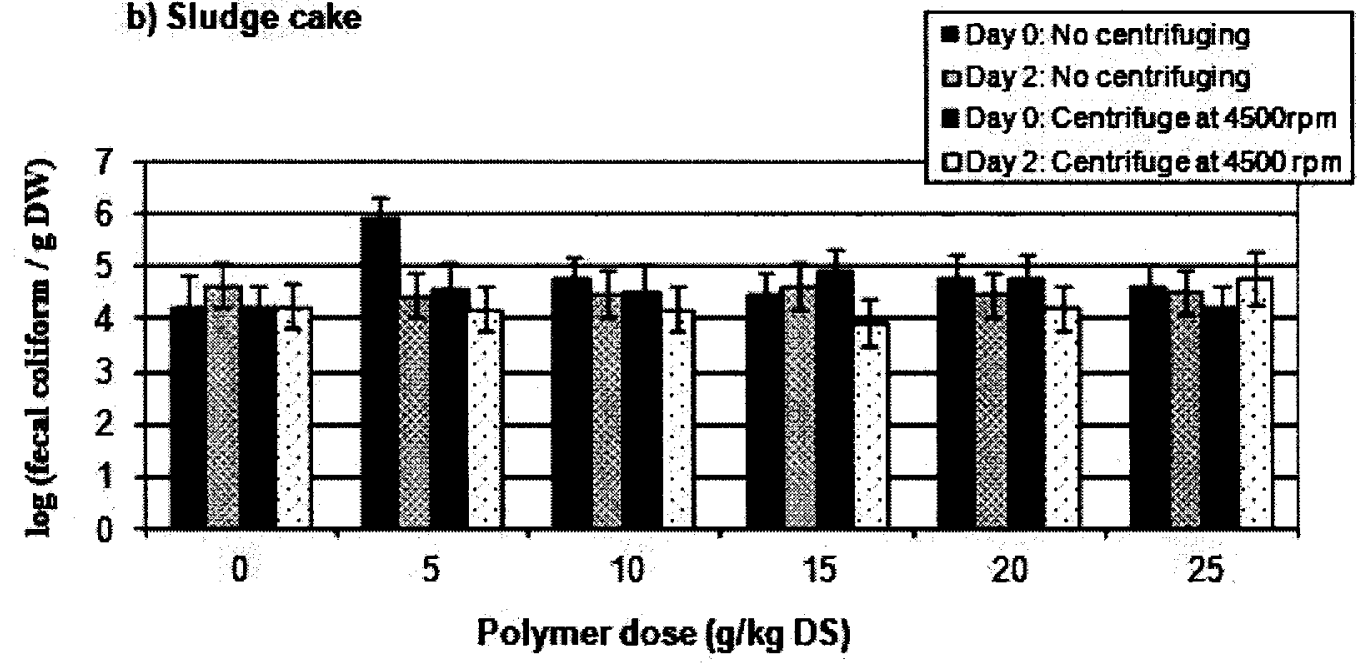

Figure 5.4: Effect of polymer dose and aging on fecal coliforms. Detection and enumeration by A-1 medium and aging for 48 hours \pm 3 hours at $24^{\circ} \mathrm{C} \pm 1^{\circ} \mathrm{C}$. Digested sludge from Treatment Plant A, Centra GP8 centrifuge run at $4500 \mathrm{rpm}, \mathrm{CA} 4600$ polymer, optimum dose: $15 \mathrm{~g} / \mathrm{kg}$ DS. 
Neither the centrate samples nor the sludge cake samples were affected by the addition of the CA4600 polymer at any of the doses under these experimental conditions $(p=0.16$ for centrate and $\mathrm{p}=0.97$ for sludge samples). Also, there was no apparent regrowth of the fecal coliforms by placing them at room temperature for 48 hours $(p=0.36$ for centrate and $\mathrm{p}=0.25$ for sludge cake samples). The aging of the samples generally led to a decrease in the fecal coliform counts, however, these changes were slight and as such were not significant. The three highest increases from the digested sludge sample levels were for samples that were not centrifuged or aged. The centrate samples at 5 and $15 \mathrm{~g} / \mathrm{kg}$ DS and cake after $5 \mathrm{~g} / \mathrm{kg}$ DS polymer addition. There was no trend to be found in the data and the levels of fecal coliforms did not change significantly with aging at room temperature and with centrifugation. It can be stated that for the CA 4600 polymer, using the Centra GP8 centrifuge does not have a significant effect on the fecal coliform counts.

The solids concentrations using the Centra GP8 centrifuge were still low even after the addition of the polymer. As well, that centrifuge used was not refrigerated and could in turn cause undue error on the levels of fecal coliforms. Because of these reasons, the test run for the CA4600 polymer was redone this time using a Sorval RC-5B, a centrifuge that was refrigerated and could reach speeds of $10,000 \mathrm{rpm}(15,180 \mathrm{x} \mathrm{g})$.

The second sampling run for Test \#2 was performed with the same polymer as the first sampling event but used the Sorval centrifuge at $10,000 \mathrm{rpm}$. The optimum polymer dose in this case was $17 \mathrm{~g} / \mathrm{kg}$ DS, calculated using the CST test and rheograms. The polymer 
doses which were tested were: 10, 13, 17, 20 and $23 \mathrm{~g} / \mathrm{kg}$ DS. Fecal coliform levels for the centrate samples are given in Figure 5.5a and the MPN values for the sludge cake samples are given in Figure 5.5b. The sludge cake solids concentrations were higher using this centrifuge, with cake samples after optimum dose polymer addition and after centrifugation having $17-19 \%$ solids, compared to $13-15 \%$ with the Centra GP8 centrifuge. The polymer dose did not have an effect on the fecal coliform counts using the higher speed centrifuge $(p=0.43)$. For the samples that were not run through the centrifuge, polymer dose had a slight effect on the fecal coliform counts $(p=0.05)$. No trend, such as higher dose leading to higher fecal coliform counts was observed.

One thing that can be noted from Figure $5.5 \mathrm{~b}$ is that the regrowth of fecal coliforms after aging at room temperature, although still small, occurred almost exclusively in the cake samples that were run through the centrifuge. This regrowth led to a significant increase in fecal coliforms at the higher doses calculated using an ANOVA $(\mathrm{p}=0.04)$. The error bars on the graph are given by the MPN calculator and do not coincide with significance levels from the statistics that were performed. It is for this reason that the change is significant even though the error bars on Figure 5.5b are overlapping. Those that were not run through the centrifuge either had a drop in their levels of fecal coliforms or stayed relatively stable. The LSD statistic is a post-hoc test which can be undertaken after an ANOVA test shows that a variance component has a significant effect. The test compares all of the means of the polymer doses individually to see which of the means is causing the effect on the variance. In this case, the test showed that the levels of fecal coliforms at $17 \mathrm{~g} / \mathrm{kg}$ DS were significantly higher than those at $10 \mathrm{~g} / \mathrm{kg} \mathrm{DS}(\mathrm{p}=0.045)$ and that the $\mathrm{p}$ - 
values were also low for the polymer doses of $13 \mathrm{~g} / \mathrm{kg} \mathrm{DS}(\mathrm{p}=0.06)$ as well as at $35 \mathrm{~g} / \mathrm{kg}$ $\mathrm{DS}$ and $0 \mathrm{~g} / \mathrm{kg}$ DS (both at $\mathrm{p}=0.07$ ) even though the $\mathrm{p}$-values are $>0.05$.

CA 4600 polymer and aging a) Centrate

Day 0: No centrifuging 由 Day 2: No centrifuging

Day 0: Centrifuge at $10000 \mathrm{rpm}$
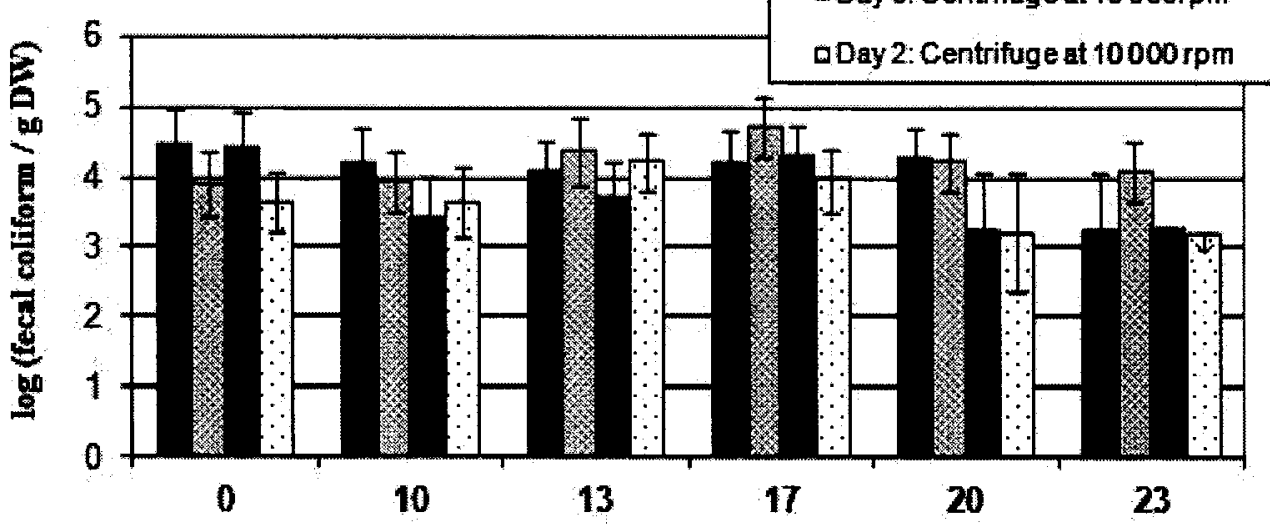

Polymer dose (g/ kg DS)

CA 4600 polymer and aging b) Sludge cake
- Day 0: No centrifuging

Day 2 : No centrifuging

Day 0: Centrifuge at $10000 \mathrm{rpm}$

oDay 2: Centrifuge at $10000 \mathrm{rom}$

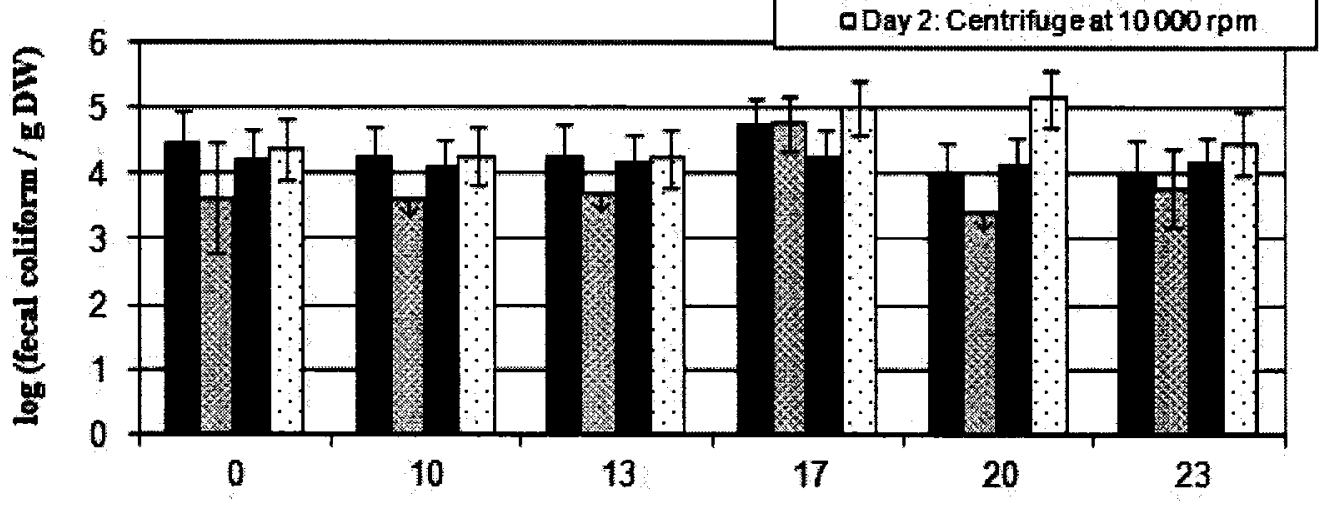

Polymer dose (g/kg DS)

Figure 5.5: Effect of polymer dose and aging on fecal coliforms. Detection and enumeration by $\mathrm{A}-1$ medium and aging for 48 hours \pm 3 hours at $24^{\circ} \mathrm{C} \pm 1^{\circ} \mathrm{C}$. Digested sludge from Treatment Plant A, Sorval RC-5B centrifuge run at $10000 \mathrm{rpm}, \mathrm{CA} 4600$ polymer, optimum dose: $17 \mathrm{~g} / \mathrm{kg}$ DS. 
Samples of sludge cake with a polymer addition of $17 \mathrm{~g} / \mathrm{kg}$ DS and $20 \mathrm{~g} / \mathrm{kg}$ DS show increases in fecal coliforms of $\log 0.76$ and $\log 1.02$ respectively after 48 hours at room temperature. This regrowth only occurred for samples with the highest solids concentrations. Work has shown that regrowth occurs more often for higher solids centrifuges, which would give higher cake solids concentrations. This observation and the possibility that the cake solids concentrations as well as the absence of centrate in the cake samples could have an effect on fecal coliform counts is tested further in Test 2 of Phase II of the project: Effect of time and temperature (Section 5.2.2).

A different polymer was used for the next run of samples. An emulsion polymer (SD2061) with the same degree of charge but a lower molecular weight was chosen to undertake this sample run. A different method for mixing the polymer was used as the previous polymer was obtained in powder form. The optimum polymer dose was calculated using the CST method and the rheogram method. The polymer dose was found in terms of $\mathrm{ml}$ of polymer per $150 \mathrm{ml}$ of sludge and was then converted and given in terms of $\mathrm{g} / \mathrm{kg}$ DS.

The optimum dose for the SD2061 polymer was much higher than for the CA 4600 polymer, at $55 \mathrm{~g} / \mathrm{kg}$ DS. The polymer doses which were tested were: $40,47,55,60$ and $67 \mathrm{~g} / \mathrm{kg}$ DS. Results from the SD2061 polymer for centrate and sludge cake MPN values are given in Figures 5.6a and 5.6b, respectively. This polymer, although having a higher optimum polymer dose, led to a much cleaner centrate, with a lower solid concentration. 
The solid concentrations in the sludge cake samples, however, were lower than those obtained from the CA 4600 polymer, the highest solids concentrations being $16-17 \%$.
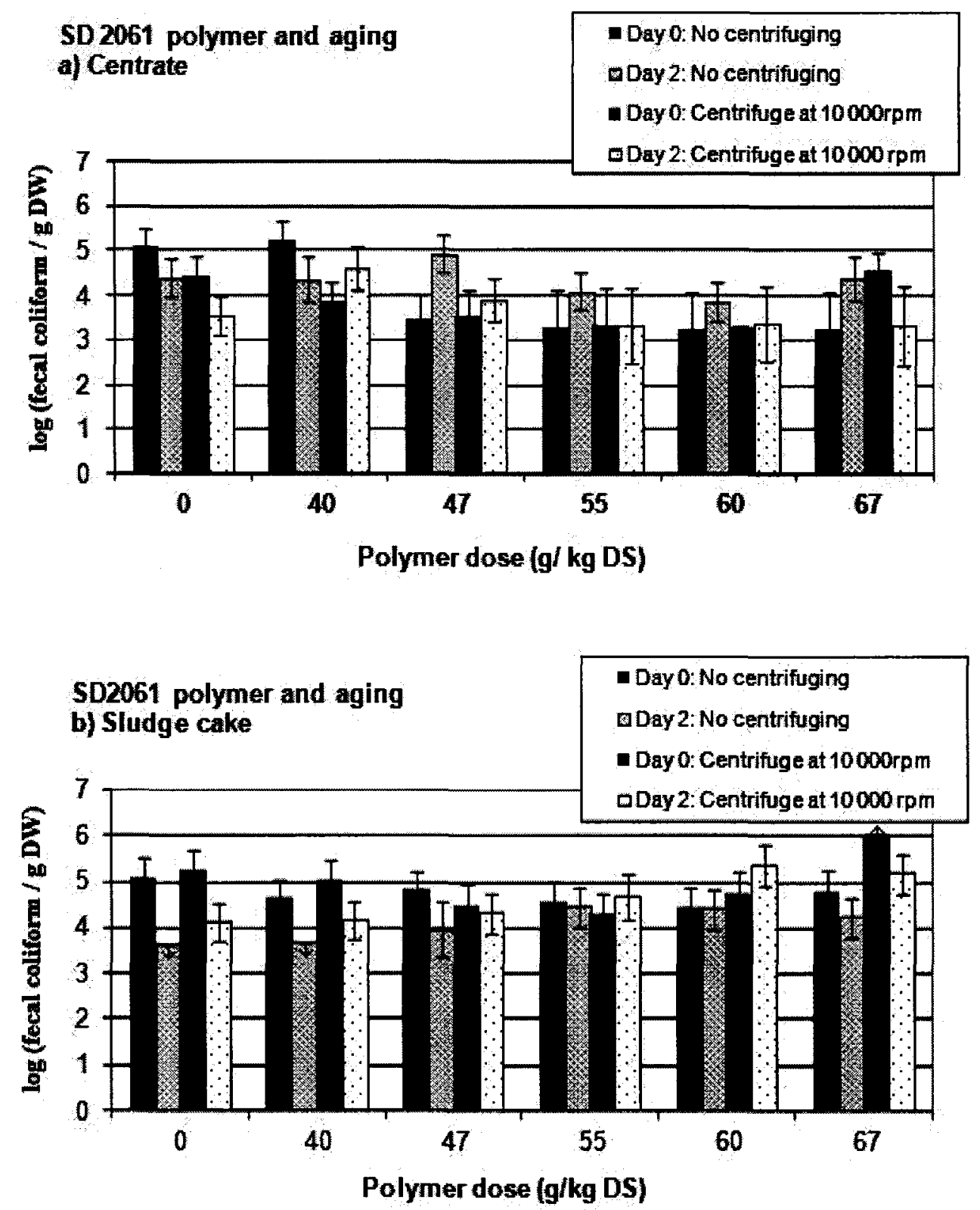

Figure 5.6: Effect of polymer dose and aging on fecal coliforms. Detection and enumeration by $\mathrm{A}-1$ medium and aging for 48 hours \pm 3 hours at $24^{\circ} \mathrm{C} \pm 1^{\circ} \mathrm{C}$. Digested sludge from Treatment Plant A, Sorval RC-5B centrifuge run at $10000 \mathrm{rpm}, \mathrm{SD} 2061$ polymer, optimum dose: $55 \mathrm{~g} / \mathrm{kg}$ DS. 
Because the centrate from the centrifuge samples was so clean, the log MPN values for those samples were quite low and the cake samples had higher levels of fecal coliforms in comparison. Regrowth was observed on a few sludge cake samples but no statistically significant effect was observed from the ANOVA test $(p=0.31)$. No statistically significant effect was observed for the ANOVA test on the effect of the polymer dose $(\mathrm{p}=0.40)$. However, the well overdosed sludge sample $(67 \mathrm{~g} / \mathrm{kg} \mathrm{DS})$ had a high level of fecal coliforms reaching six log. A one sample t-test compares a value with the means of all the other polymer doses. This test was undertaken for the fecal coliform value of the well overdosed sample. It was found that the value was significantly higher than the fecal coliform counts from the other concentrations for all other polymer doses $(p<0.001)$. These observations are consistent with those from Qi et al. (2007) who added polymer to digested sludge and found that it could increase the fecal coliform counts. The weight ratio of polymer to sludge is 1:15 in the current study, and significant changes occurred in previous studies for ratios of 1:10 (Qi et al., 2007). The added nutrients using this polymer could be the reason for the rise in fecal coliforms. The optimum polymer dose of the SD2061 polymer is much higher than for the CA4600 polymer. The concentration of polymer used at Treatment Plant $\mathrm{A}$ is lower than the optimum doses found for the study, dosing at $8.4 \mathrm{~g} / \mathrm{kg}$ DS compared to the optimum dose of 15 and $17 \mathrm{~g} / \mathrm{kg}$ DS. The sludge will likely be underdosed at the treatment plant. 


\subsubsection{Simulation of full-scale centrifuges in the lab}

The tests performed in Phase I of the project could only have been done using a laboratory centrifuge, testing the effect of different centrifuge speeds and different doses would not have been possible in a full-scale setting. The challenge with working in a laboratory setting is that there is currently no standard protocol for the simulation of the centrifuges with lab-scale tests. The low solids concentrations obtained in the cake samples have been described earlier in the paper and have also been observed by other studies which attempted to recreate treatment plant dewatering in a laboratory (Iranpour et al., 2005).

The main difference which could affect the amount and the type of shear in the system is the manner in which the solids and the liquids portions of the samples are separated. A swinging bucket or a fixed angle centrifuge has the solids accumulating in the bottom of the centrifuge jars through the high centrifugal acceleration. In a full-scale setting, although the relative centrifugal force for the device is the same, the solids and the liquid do not separate in the same manner. As was shown in Figure 1.1, the solids in a full-scale dewatering centrifuge accumulate on the edges of the centrifuge and are then pushed forward with the action of the screw conveyor to be discharged at the feed end of the centrifuge. The centrate is separated from the sludge cake on an ongoing basis during centrifugation and this centrate is discharged from the center of the solid bowl. 
At similar relative centrifugal accelerations, the laboratory centrifuge cannot produce cake with as high a solid concentration as dewatering centrifuges. Although preliminary studies have shown that at Treatment Plant A, regrowth of fecal coliforms is possible, the laboratory tests did not show any increases. This could simply be due to the centrifuge not being powerful enough to separate the solids from the liquids or it could be due to the differences in the shear added to the system. The characteristics of the sludge cake and the centrate obtained using these two types of centrifuges are therefore also different. The characterisation of the centrate obtained through a lab-scale centrifuge as compared with a full-scale centrifuge will be examined further in Phase II of this project (starting in Section 5.2.3.). There is currently no protocol for the simulation of a full-scale centrifuge in a laboratory setting or an appropriate scale-down model for dewatering devices. Until a protocol is written, the challenges with using a laboratory setting will remain. 


\subsection{Phase II}

The goal of Phase II was to examine if reactivation or regrowth was possible in two fullscale treatment plants. It was split into five different tests; methodology has been laid out in Section 4.2. These tests focused on changes within the treatment plant, aging of samples, centrate characterisation and the differences between total viable cells and culturable fecal coliform counts. For these tests, lab-scale samples were compared with full-scale samples as their characteristics would be different.

\subsubsection{Test 1: Full-scale samples}

In Test 1, sludge from two different treatment plants was analysed. Treatment Plant A, which was used for the Phase I tests used a high-solids centrifuge that produced cake solid averages ranging from $30-35 \%$ and Treatment Plant $\mathrm{B}$ had a low-solids centrifuge that generated cake solids ranging from $18-20 \%$. Their characteristics were outlined in Table 1.2 and Table 4.1. It was the goal of this test to compare the changes in fecal coliforms within the treatment plants. Besides the difference with the centrifuges that they use, the polymers used for these treatment plants were different, both of them were dry polymers and had an ultra high molecular weight but the polymer used for Treatment Plant B, CB4350, had a smaller degree of cationic charge, $15 \%$ as opposed to $40 \%$ for the CA4600 used in Treatment Plant A.

Treatment Plant B used secondary effluent to make-up their polymer, accordingly, fecal coliforms could be present in the polymer solution and thus the levels could increase 
upon its addition. Treatment Plant A, on the other hand, used tap water to make-up their polymer. Historical data from Treatment plant A has shown that the reactivation or regrowth of $E$ coli in the cake samples post-dewatering has been observed (Figure 1.3). Past data from on-site testing for fecal coliforms from Treatment Plant B could not be obtained. Because of this, it was not known if this treatment plant would show postdewatering increases as Treatment Plant A did. As recovery was seen more with highsolids centrifuges (Qi et al., 2004), Treatment Plant A was expected to show larger increases than Treatment Plant B.

Sampling on two different days at Treatment Plant A was undertaken as the characteristics of the sludge could change with time because of the heterogeneity of sludge. The results from run $\# 1$ and run \#2 are given in Figures 5.7a and 5.7b respectively. Both graphs have very similar patterns throughout the treatment train. The sludge going into the digester has very high fecal coliform levels. Upon exiting the digester, the MPN values for fecal coliforms drop and stay relatively stable in the remainder of the treatment plant. For both runs, the addition of polymer did not increase or decrease the levels of fecal coliforms in the digested sludge and there was a slight increase in the fecal coliforms after mechanical dewatering by centrifuge. 
Full-scale Treatment Plant A

a) Run \#1

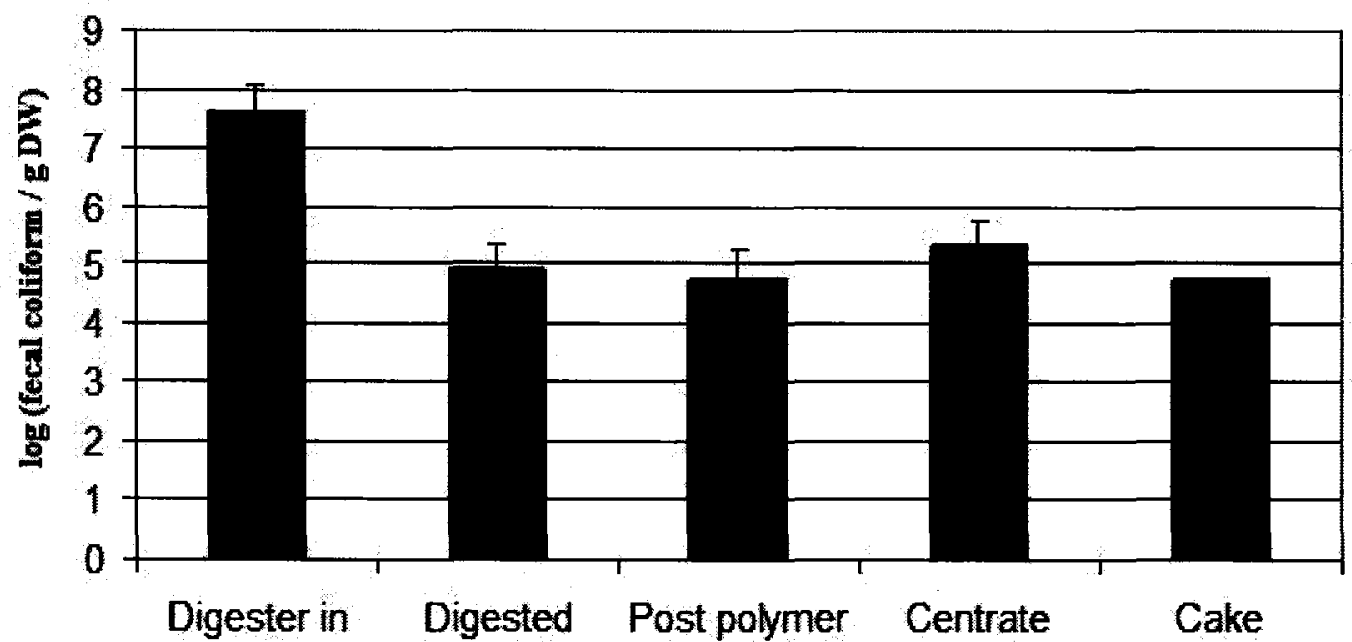

Full-scale Treatment Plant A

b) Run \#2

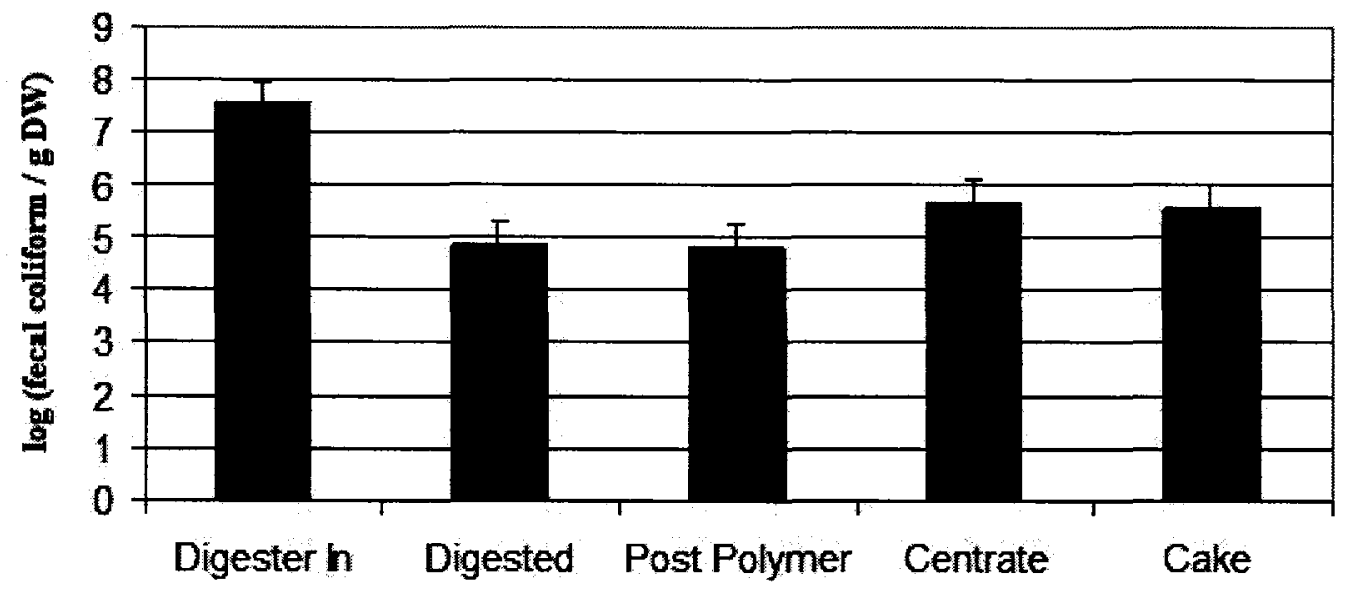

Figure 5.7: Fecal coliform levels throughout sludge treatment train at Treatment Plant A. Detection and enumeration by A-1 medium.

The results from the full-scale sampling at Treatment Plant B are given in Figure 5.8. For this treatment plant, an extra sampling site was added as the plant dries their dewatered sludge cake which leads to pellets with solids concentrations above $95 \%$. These pellets 
have extremely low fecal coliform levels (<log three) but still have the nitrogen, phosphorous and carbon that can make them ideal as fertilizer. More information on the technology has been outlined in Section 1.4. For this treatment plant, even with their polymer being mixed with secondary effluent, the levels of fecal coliforms show very little post polymer increase. There are slight increases after centrifuge dewatering, in both the centrate and the cake. As would be expected, the pellets have very low levels of fecal coliforms; the high temperatures during the drying process would inactivate the fecal coliforms in the cake.

\section{Full-scale Treatment Plant B}

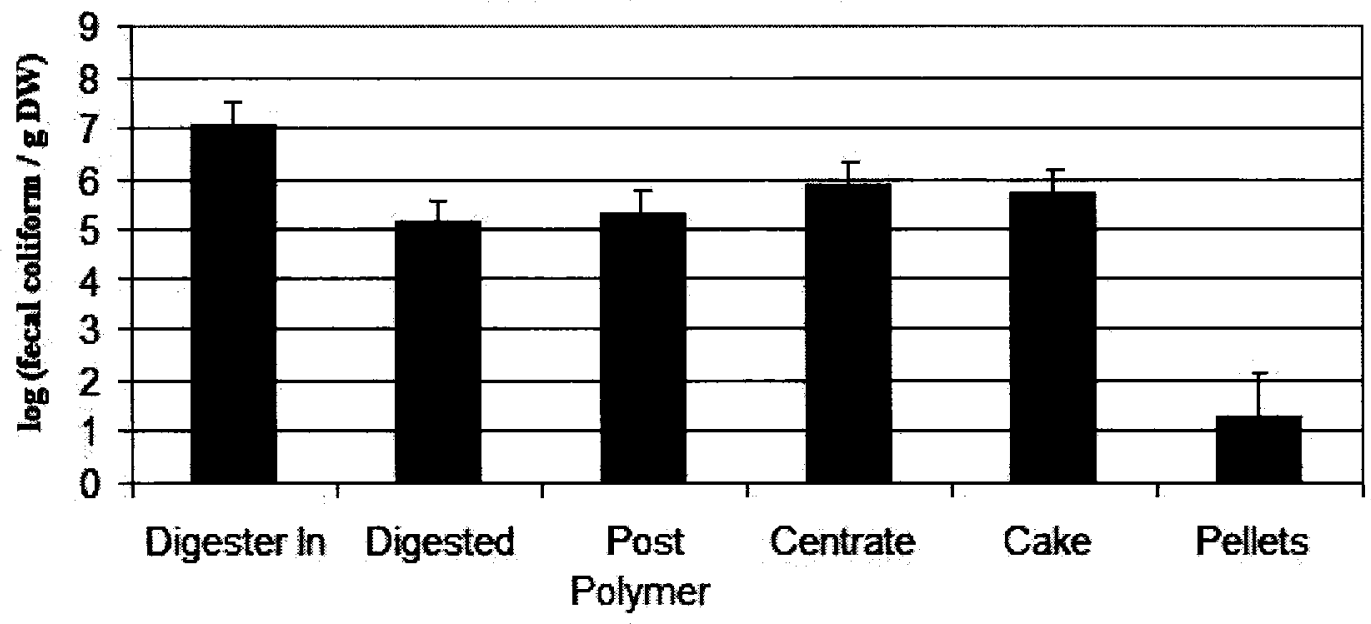

Figure 5.8: Fecal coliform levels throughout sludge treatment train at Treatment Plant B. Detection and enumeration by A-1 medium.

While undertaking the full-scale sampling and enumeration of the fecal coliforms, some preliminary testing of the effects of aging the dewatered sludge samples was also undertaken. As results from Treatment Plant A showed higher levels in the sludge cake than the digested sludge (Figure 1.3), it was hypothesised that the bacteria in the sludge 
had the possibility of reactivation and / or regrowth. Reactivation was not observed immediately following centrifuge dewatering but the regrowth of fecal coliforms in the sludge remained a possibility. After the first sampling at Treatment Plant A, samples of cake were placed at both $4^{\circ} \mathrm{C} \pm 0.5^{\circ} \mathrm{C}$ and $24^{\circ} \mathrm{C} \pm 1^{\circ} \mathrm{C}$ and left for seven days. After seven days, enumeration was undertaken and the sample from the refrigerator was placed at room temperature for a further seven days. The results of these preliminary tests are given in Figure 5.9.

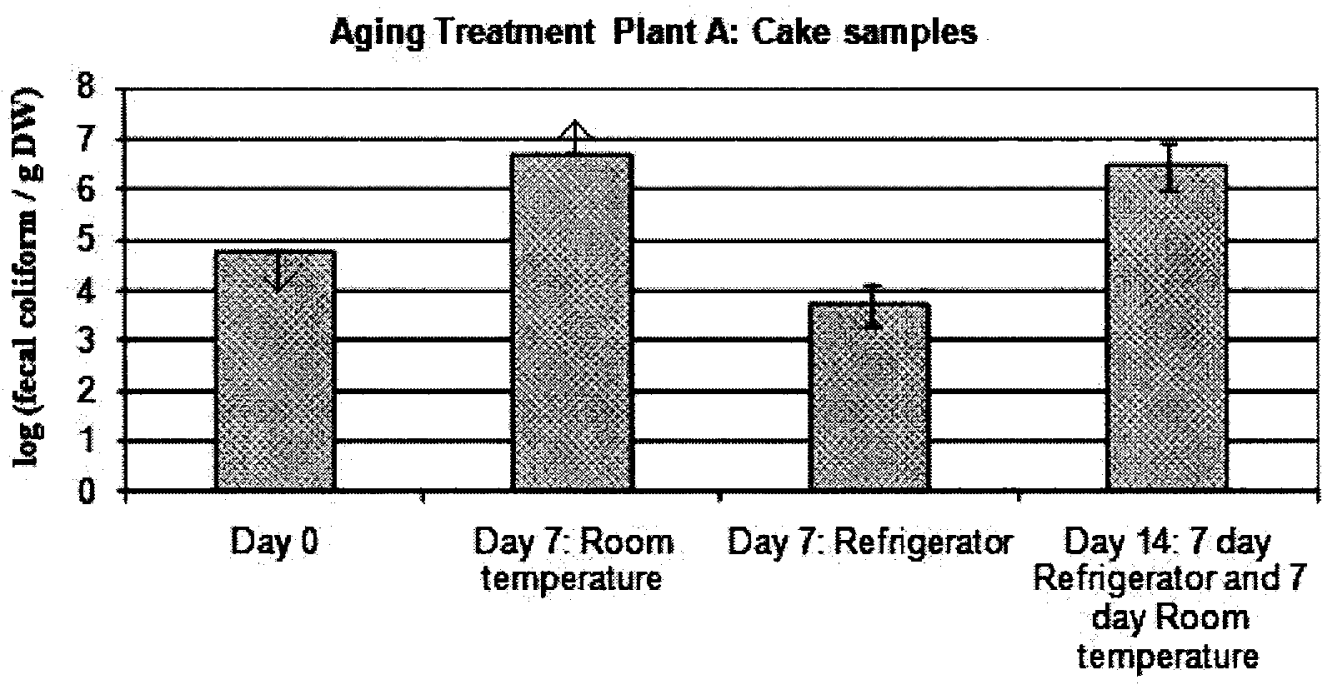

Figure 5.9: Fecal coliform levels in cake samples at Treatment Plant A. Room temperature $=24^{\circ} \mathrm{C} \pm 1^{\circ} \mathrm{C}$, Refrigerator $=4^{\circ} \mathrm{C} \pm 0.5^{\circ} \mathrm{C}$. Detection and enumeration done by A-1 medium.

Within the seven days at room temperature, the fecal coliforms increased by $1.92 \log$. However, for the sample that was placed in the refrigerator, the fecal coliform levels dropped by $1.05 \mathrm{log}$. Once the refrigerator sample was removed from there and was allowed to age at room temperature again, its fecal coliform counts increased to a level that was similar to the sample which was never placed in the refrigerator. What this 
shows is that the levels of fecal coliforms in the sludge cake at that treatment plant can be affected by time and temperature. The samples which were in the refrigerator were able to recover and increase to the same level as a sample which was never placed in the refrigerator.

A similar preliminary test was undertaken for the sludge cake at Treatment Plant B with samples placed at room temperature and in the refrigerator for four days. Cake samples were placed at both temperatures and sludge pellet samples were placed at room temperature in order to see if the fecal coliforms had in fact become nonviable by the heat treatment. The results of these tests are given in Figure 5.10.

Aging Treatment Plant B: Cake and Pellets

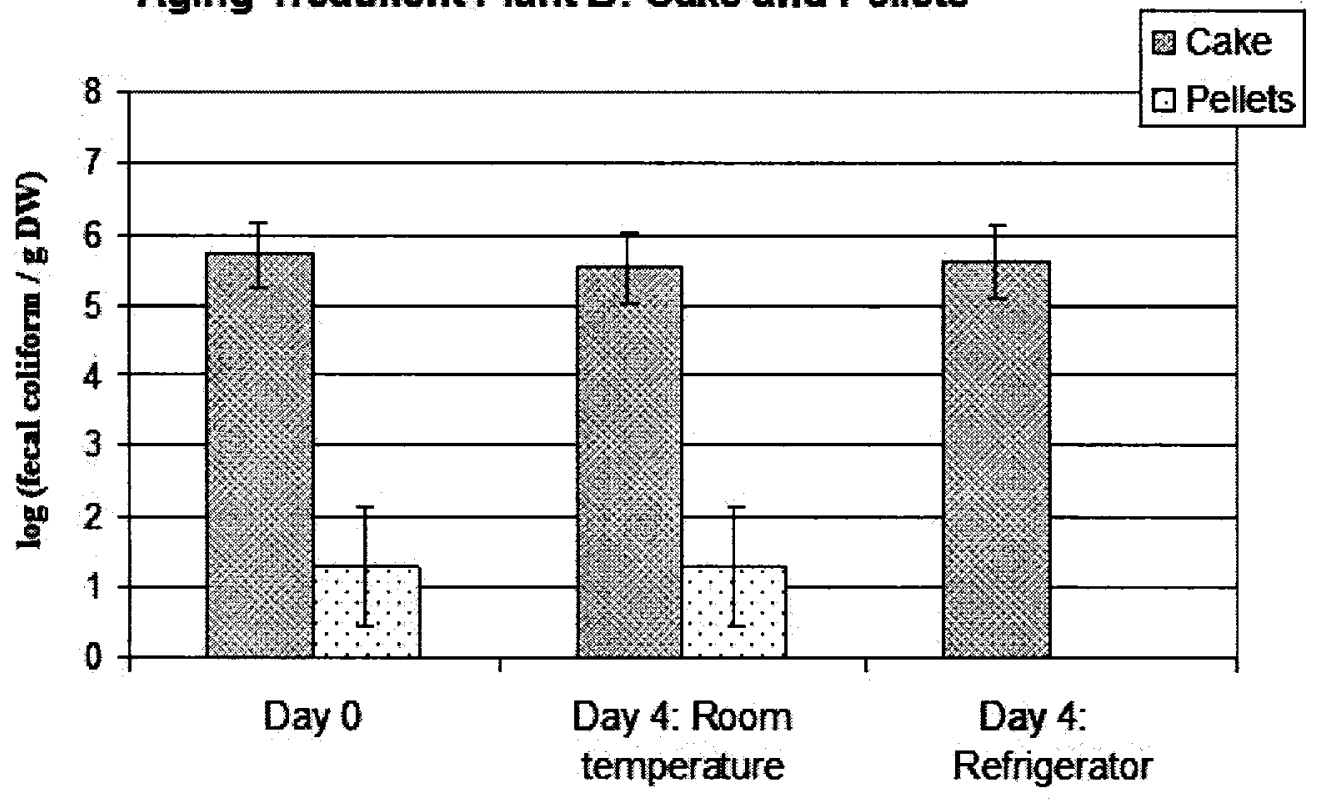

Figure 5.10: Fecal coliform levels in cake and pellet samples at Treatment Plant B. Room temperature $=24^{\circ} \mathrm{C} \pm 1{ }^{\circ} \mathrm{C}$, Refrigerator $=4^{\circ} \mathrm{C} \pm 0.5^{\circ} \mathrm{C}$. Detection and enumeration done by A-1 medium. 
The fecal coliform levels in the cake at Treatment Plant B do not show any of the changes that were observed at Treatment Plant A. The levels stay relatively steady in the cake and the pellets, with no increase at room temperature and no decrease for samples placed in the refrigerator.

\subsubsection{Test 2: Cake and centrate sample aging at different temperatures}

The observations from the previous test were that no immediate changes occurred postdewatering at either of the treatment plants studied. However, at Treatment Plant A, recovery of fecal coliforms in cake samples at certain temperatures was possible. This led to further tests on the aging of samples at different temperatures. Samples were collected and put together as outlined in Figure 4.2. Samples that were mixed were: digested sludge, cake, centrate, cake + phosphate buffered solution (PBS) and cake + centrate. These samples were either obtained directly from the treatment plant and mixed together in the laboratory or they were produced by the centrifugation of the digested sludge in the laboratory centrifuge, run at 10,000 rpm. For each treatment plant, the results are split up into two figures. The first figure shows the results for the full-scale samples of cake and centrate with the three different temperatures in separate windows; a) refrigerator, b) room temperature, c) incubator. The second graph contains the samples of cake and centrate that were obtained from the laboratory centrifuge but mixed in the same way as the full-scale samples and with the same temperatures being studied for the experiment. Enumeration of fecal coliforms was done directly after sampling as well as after 2 and 4 days of aging at these different temperatures. 
The results from the full-scale sample aging at Treatment Plant A can be found in Figure 5.11 , with a) refrigerator, b) room temperature and c) incubator. The fecal coliform levels in the refrigerator samples (Figure 5.11a) stay relatively stable during the extent of the testing period. At this temperature, no significant change in the fecal coliforms was seen with aging $(\mathrm{p}=0.60)$. The cake + PBS samples and the cake + centrate samples do drop at Day 4 after increasing at Day 2.

For the room temperature results on Figure $5.11 \mathrm{~b}$, increases in fecal coliform counts are observed for certain samples. ANOVA tests showed that the effect of the sample type on the fecal coliform counts is significant to a $10 \% \mathrm{p}$-value $(\mathrm{p}=0.09)$. The increases in culture counts are mostly for the cake and the cake + PBS samples, the two samples which do not contain centrate. Although the samples of cake + centrate do rise after two days, they drop back down at Day four. Within the first two days of sample aging at room temperature, the full-scale cake was able to increase in fecal coliforms by log 2.4 and the cake + PBS sample was able to increase in fecal coliforms by $\log 3.4$. As the solids concentration for the cake + PBS and the centrate + cake samples were quite similar, the main difference between these two samples was the presence or absence of the centrate in the sample. 

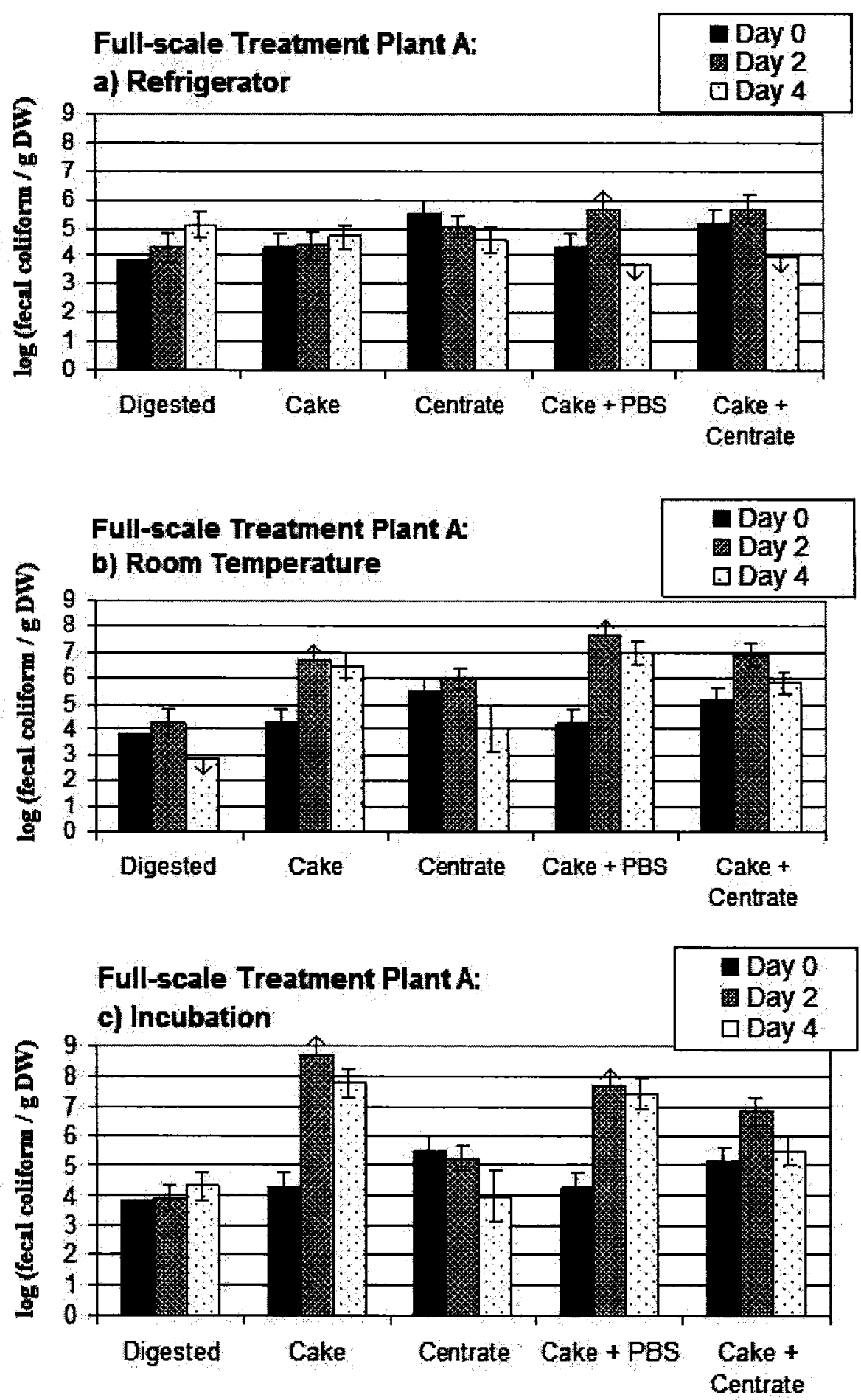

Figure 5.11: Fecal coliform levels in full-scale Treatment Plant A samples. Refrigerator $=4^{\circ} \mathrm{C} \pm 0.5^{\circ} \mathrm{C}$, room temperature $=24^{\circ} \mathrm{C} \pm 1^{\circ} \mathrm{C}$, incubator $=35^{\circ} \mathrm{C} \pm 0.5^{\circ} \mathrm{C}$. Detection and enumeration done by A-1 medium. 
After the ANOVAs were performed, the LSD test was undertaken in order to see if the variance was caused by certain samples over others. For the samples aged at room temperature, the test showed that the change in fecal coliforms for the cake + PBS samples was significantly higher than for digested sludge $(\mathrm{p}=0.033)$ as well as the centrate $(\mathrm{p}=0.027)$. At this temperature, the increase in fecal coliforms in the cake was not significantly higher than any of the other samples and the decrease in the fecal coliforms from the centrate samples was not significantly lower than any samples except for the cake + PBS samples.

The general trend observed for the samples placed at room temperature is similar for those samples which were placed in the incubator, see Figure 5.11c. The main difference at this temperature is that the increases are greater for the cake samples and the cake + PBS samples. The ANOVA test shows a degree of significance for the samples at 0.02 . Within the first two days, fecal coliforms in the cake samples increase by $\log 4.4$ and the cake + PBS sample by $\log 3.4$. On the other hand, the samples of digested sludge stayed stable for the duration of the test and the full-scale centrate samples showed a decrease in fecal coliforms when placed in the incubator for four days, decreasing by $\log 1.6$ within the four days of the test. Although the centrate + cake samples do increase, their numbers drop again after four days in the incubator.

The results from the aging of full-scale Treatment Plant A sludge point to the presence of an inhibitory substance in the centrate because growth of fecal coliforms was much greater in the two samples for which centrate was not present at room temperature and in 
the incubator. Digested sludge samples, centrate samples and cake + centrate samples on the other hand, do not rise, they either drop or stay relatively stable. As was observed at room temperature, the cake + centrate sample does rise after two days of aging but then falls back down to levels similar to that of day zero at day four. LSD statistics show that the change in fecal coliforms for the cake samples was significantly higher than the change for the digested samples $(p=0.011)$, the centrate samples $(0.004)$ and the cake + centrate samples $(\mathrm{p}=0.030)$. For the cake + PBS samples, the levels were significantly higher than the digested samples $(p=0.026)$ and centrate $(p=0.007)$, however, it was not significantly higher than the cake + centrate samples $(\mathrm{p}=0.078)$, this would be due to the increase in the cake + centrate sample at day two.

The samples which were run through the lab-scale centrifuge using Treatment Plant A digested sludge did not show the same trend as those obtained from the treatment plant itself. The results are given in Figures $5.12 \mathrm{a}, \mathrm{b}$ and $\mathrm{c}$ for the refrigerator, room temperature and incubator, respectively. Changes are minimal regardless of the temperature; while some samples show a slight increase or decrease, the change in fecal coliform levels is not significant for the refrigerator $(\mathrm{p}=0.197)$, at room temperature $(p=0.938)$ or the Incubator $(p=0.860)$, calculated with the ANOVA test. Also, LSD tests show no difference between any of the means of the samples at any of the temperatures taken separately. 

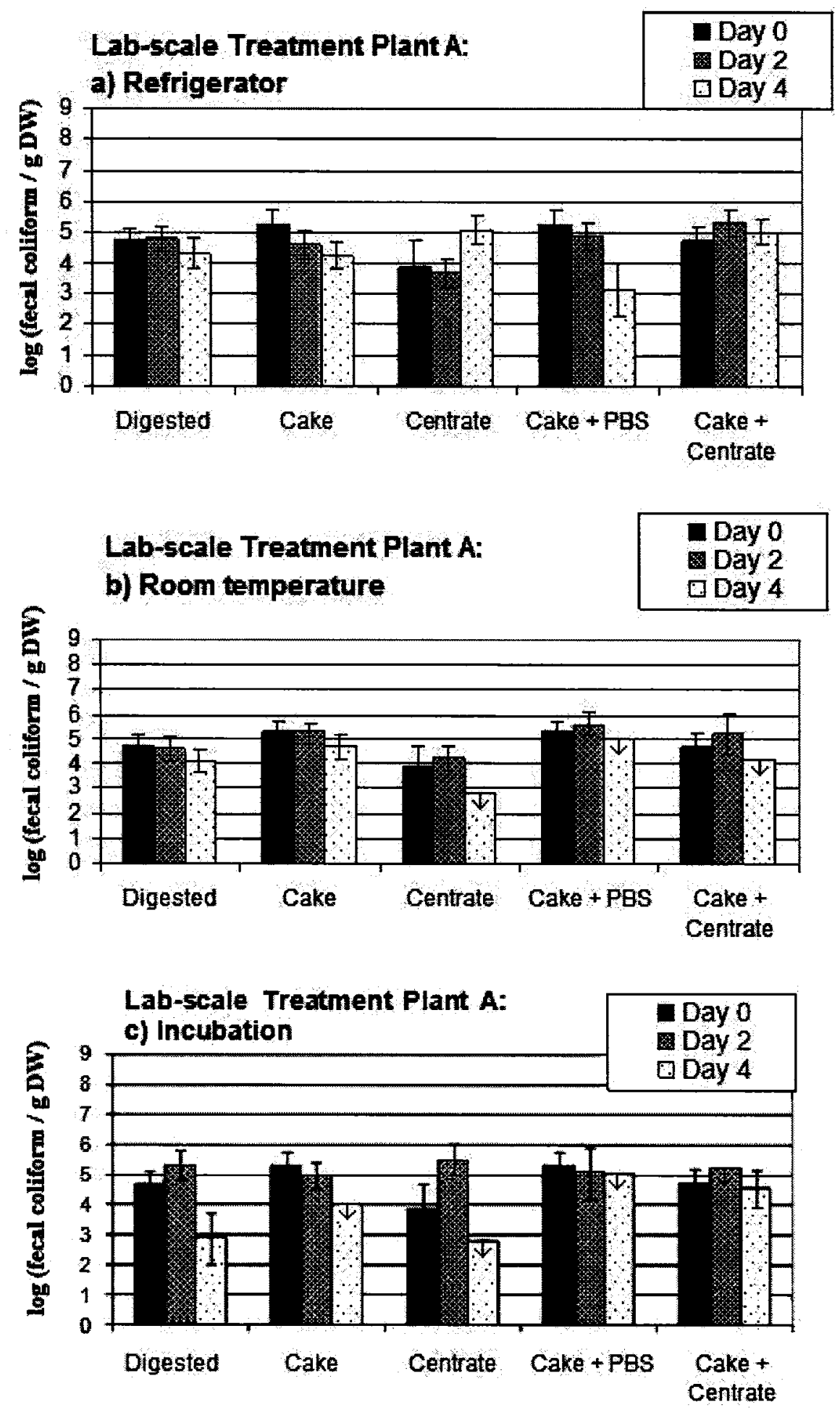

Figure 5.12: Fecal coliform levels for lab-scale samples with Treatment Plant A digested sludge. Refrigerator $=4^{\circ} \mathrm{C} \pm 0.5^{\circ} \mathrm{C}$, room temperature $=24^{\circ} \mathrm{C} \pm 1^{\circ} \mathrm{C}$, incubator $=35^{\circ} \mathrm{C}$ $\pm 0.5^{\circ} \mathrm{C}$. Detection and enumeration done by A-1 medium. 
The centrate placed in the refrigerator did have an increase of one order of magnitude after four days (Figure 5.12a) whereas at room temperature and in the incubator, the fecal coliforms decreased to such a degree that no growth was observed at its lowest dilution of $1 / 10$ on day four (Figure $5.12 \mathrm{~b}$ and Figure 5.12c). No inhibitory growth effect of the labscale centrate was observed as the samples of cake and cake + PBS did not increase. The cake samples at all temperatures showed a decrease in fecal coliform counts. This shows us that the characteristics of the centrate and the cake obtained from the lab are quite different than those that are obtained from the treatment plant itself. The possible inhibitory substance removed during full-scale centrifugation into the centrate would not be removed here and could still be present in the cake samples leading to decreases in fecal coliform counts.

Fecal coliform data could not be obtained from Treatment Plant B itself. As the plant turns their cake into pellets before shipping them, there is little need for them to monitor fecal coliforms in their dewatered cake as it will not be land applied. Preliminary observations of the aging of the cake samples, showed no regrowth after four days at room temperature. It was for this reason that the results from this treatment plant were not expected to be as dramatic as those for Treatment Plant A. Full-scale results for Treatment Plant B are given in Figure 5.13a, $\mathrm{b}$ and $\mathrm{c}$. 

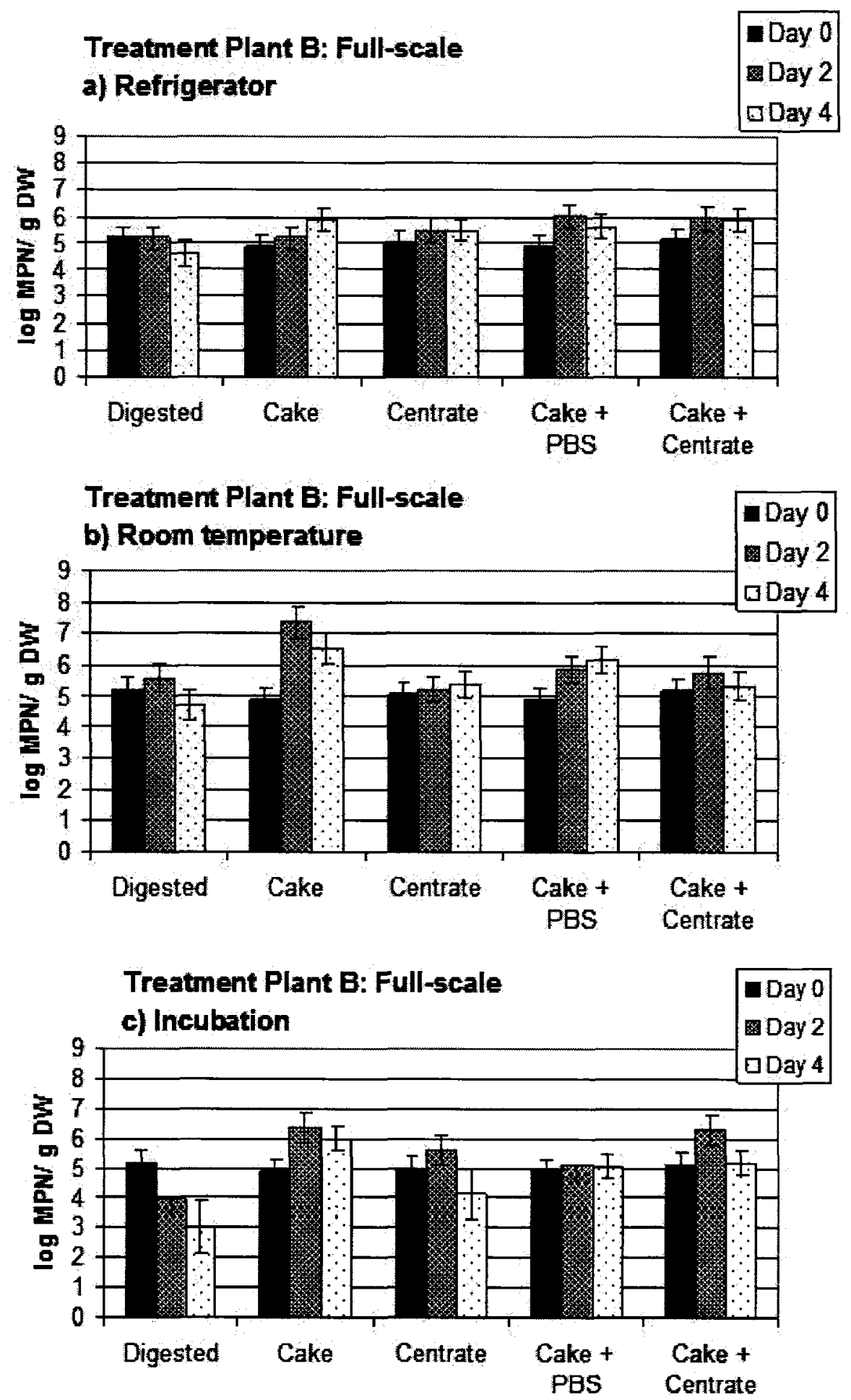

Figure 5.13: Fecal coliform levels for full-scale samples with Treatment Plant $B$ digested sludge. Refrigerator $=4^{\circ} \mathrm{C} \pm 0.5^{\circ} \mathrm{C}$, room temperature $=24^{\circ} \mathrm{C} \pm 1^{\circ} \mathrm{C}$, incubator $=35^{\circ} \mathrm{C}$ $\pm 0.5^{\circ} \mathrm{C}$. Detection and enumeration done by $\mathrm{A}-1$ medium 
The samples placed in the refrigerator showed little increase, while some samples decreased with aging (Figure 5.13a). The ANOVA table looking at the effect of the sample type on the fecal coliform counts gives a level of significance of 0.049 for all samples in the refrigerator. The level of significance from these samples is due mostly to the decreases in the fecal coliform counts of the digested sludge samples in the refrigerator. The LSD statistic showed that the decrease in the digested sludge sample was significantly lower than cake $(p=0.027)$, cake + centrate $(p=0.031)$ and cake + PBS $(p=0.007)$. The cake + PBS sample did increase; however, this increase was only significantly higher than the digested samples.

At room temperature, the only sample which increased with aging was the cake sample (Figure 5.13b) but this led to a significant effect of the sample type on the change in fecal coliforms with aging $(\mathrm{p}=0.030)$. The cake sample at room temperature rose by $\log 2.5$ after two days before dropping slightly at day four. The cake + PBS sample increases more than the cake and centrate samples, but this increase is less dramatic than Treatment Plant A full-scale samples. LSD statistics show that the cake increase is significantly higher than in the digested samples $(\mathrm{p}=0.005)$, the centrate samples $(\mathrm{p}=0.010)$, the cake + centrate samples $(p=0.031)$ and even the cake + PBS samples $(p=0.037)$. The increases from the cake samples at room temperature show that regrowth is possible at Treatment Plant $B$ as well and that this would pose a problem if the treatment plant did not thermally treat their dewatering sludge cake before final disposal. 
For the Treatment Plant B samples which were placed in the incubator (Figure 5.13c), cake + PBS samples stayed stable throughout the entire testing period, cake + centrate and cake samples increase and then decrease at Day 4. One important thing to not overlook at this temperature is that the fecal coliforms in the digested sludge drop after two days and even more after 4 days. This led to the digested sludge samples having a significantly lower fecal coliform counts after aging than the cake samples $(p=0.006)$, cake + centrate samples $(p=0.050)$ and cake + PBS samples $(p=0.011)$. An inhibitory substance could be present in the digested sludge samples at this treatment plant, but it may be that the centrifuge at this treatment plant is not powerful enough to remove this inhibitory substance which could be the reason that only slight increases are observed in the samples that are in the absence of the centrate.

Digested sludge from treatment plant $\mathrm{B}$ was run through the laboratory centrifuge and samples were created in the same manner as for Treatment Plant A. Figure 5.14a has the results for the refrigerator samples, like the full-scale samples from Treatment Plant B and the other treatment plant, no clear increases or decreases can be observed. The levels of fecal coliforms in the digested sludge at this temperature as well as at room temperature (Figure 5.14b) and in the incubator (Figure 5.14c) dropped with aging. As for the samples in the absence of centrate, the cake at room temperature increases by $\log 1.4$ but then dropped back down at day four. The increase was significantly different from the decrease of the digested sludge sample $(\mathrm{p}=0.015)$. 

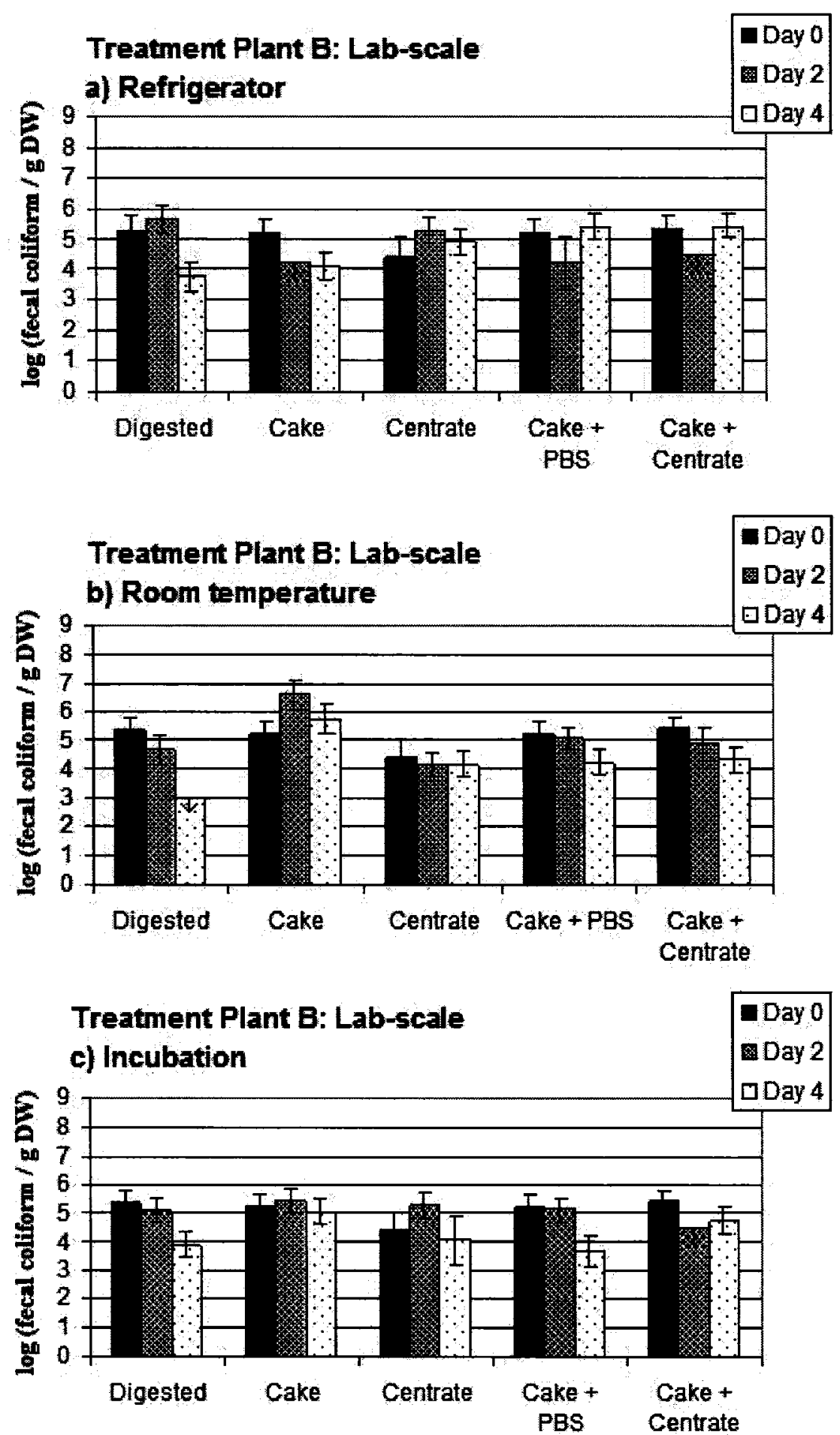

Figure 5.14: Fecal coliform levels for lab-scale samples with treatment plant $B$ digested sludge. Refrigerator $=4^{\circ} \mathrm{C} \pm 0.5^{\circ} \mathrm{C}$, room temperature $=24^{\circ} \mathrm{C} \pm 1^{\circ} \mathrm{C}$, incubator $=35^{\circ} \mathrm{C}$ $\pm 0.5^{\circ} \mathrm{C}$. Detection and enumeration done by $\mathrm{A}-1$ medium 
At both room temperature and in the incubator, the cake + PBS samples decrease with time. On the whole, the lab-scale samples showed no significant differences in their increases or decreases at all three temperatures. The p-values for the effect of the sludge samples were all higher than 0.05 ; the refrigerator $p=0.371$, room temperature $p=0.097$ and the incubator $\mathrm{p}=0.482$.

Looking at the digested sludge samples from both treatment plants shows that after four days in the incubator, there are decreases in the fecal coliform counts. The high temperature in the incubator would appear to create an inhospitable environment for the fecal coliforms in the digested sludge. To a lesser extent, other samples which include centrate also see a decrease in fecal coliforms in the incubator, with the drop being the more pronounced for full-scale Treatment Plant A centrate and cake + centrate samples than any of the other sludge treatment types.

There were four different types of cake and centrate samples studied in this test: fullscale samples from Treatment Plant A, laboratory generated samples using Treatment Plant A digested sludge, full-scale samples from Treatment Plant B and laboratory generated samples using Treatment Plant B digested sludge. The test showed that the characteristics of centrate and cake for each of these treatment types were different. Treatment Plant A, full-scale showed the most significant increases for samples in the absence of centrate, it also showed the largest decreases for the samples in the presence of centrate. However, samples at both treatment plants had decreases in fecal coliform counts in the digested sludge with time, this would lead to the theory that an inhibitory 
substance is present there and that, in the case of this study, the only centrifuge which is powerful enough to remove this substance into its centrate would be the full-scale centrifuge at Treatment Plant A. Treatment Plant B did see increases of fecal coliforms in the full-scale cake but the change was small and not significant. The centrate produced in the laboratory from either of the two treatment plants did not show any inhibitory effects on fecal coliform counts, the laboratory centrifuge would appear to not be able to extract the substances from the cake. In order to verify that the increases at Treatment Plant A were reproducible on a different sampling day, another run of Full-scale samples was undertaken (Figure 5.15).

For the second run, placing the samples in the refrigerator did not lead to a significant effect on the fecal coliform counts, $(\mathrm{p}=0.092)$, Figure $5.15 \mathrm{a}$. There was either very little change or a decrease in their numbers; with cake samples decreasing their fecal coliform numbers the most.

At room temperature, the regrowth of the bacteria in the samples without centrate was less dramatic for this second run of Treatment Plant A full-scale samples (Figure 5.15b) than those for the first run (Figure 5.11b). Despite the increases being less dramatic than those observed in the first run and outlined in Figure 5.11b, the effect of the sample type on the fecal coliform counts was statistically significant from an ANOVA test for Run \#2 $(\mathrm{p}=0.027)$. The trend was the same as for the first run, with an increase in the fecal coliforms from the cake samples at day two, increasing by $\log 2.2$, but after day four, this increase from its original values is only $\log 0.8$. Cake samples led to a significantly higher 
fecal coliform count than centrate samples $(\mathrm{p}=0.003)$ and cake + centrate samples $(p=0.028)$. The cake + PBS samples increase only slightly after day two and to a greater degree after day four. The levels of fecal coliforms were significantly higher than the centrate samples $(\mathrm{p}=0.032)$ only. Samples of digested sludge, centrate and cake + centrate show slight decreases after two and four days at room temperature.

For the samples of the second run placed in the incubator (Figure 5.15c), the effect was similar to that of the room temperature ones and a significant change between the different samples was observed with aging $(\mathrm{p}=0.012)$. The largest increases were observed for cake + PBS samples with a $\log 1.9$ increase after two days in the incubator, fecal coliforms then dropped slightly at day four. The fecal coliform levels were still significantly higher than those in the digested samples $(\mathrm{p}=0.019)$, in the centrate samples $(p=0.002)$ and the cake + centrate samples $(p=0.007)$. Cake levels did rise but by the fourth day in the incubator, the levels dropped back down and ended up slightly lower than the original values. For this reason, the levels in the cake are only significantly higher than the levels in the centrate $(p=0.014)$ because these centrate levels decreased when placed in the incubator for two and four days. 

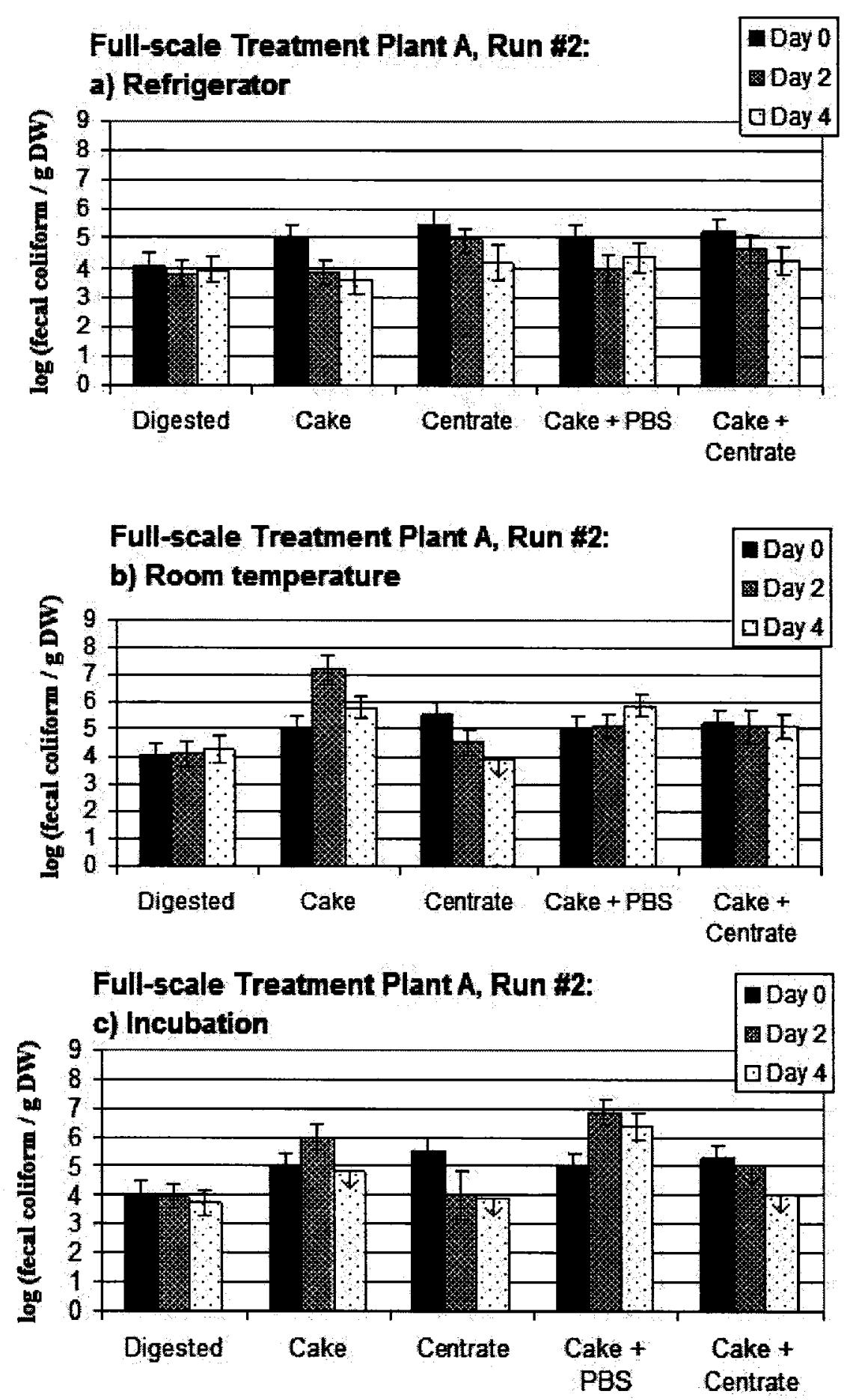

Figure 5.15: Fecal coliform levels for full-scale samples for Run \#2 from Treatment Plant A. Refrigerator $=4^{\circ} \mathrm{C} \pm 0.5^{\circ} \mathrm{C}$, room temperature $=24^{\circ} \mathrm{C} \pm 1^{\circ} \mathrm{C}$, incubator $=35^{\circ} \mathrm{C}$ $\pm 0.5^{\circ} \mathrm{C}$. Detection and enumeration done by A-1 medium. 
The results from the second run of samples are consistent with those of the first run. Increases in the samples in the absence of centrate were observed and decreases in samples that were in the presence of centrate were also observed. The changes are the most significant in the incubator; this temperature, combined with the presence of the centrate leads to a decrease in fecal coliform counts whereas in the absence of the centrate, a significant short-term regrowth of the bacteria is observed. The optimum temperature for fecal coliforms is usually found between 35 and $40^{\circ} \mathrm{C}$ (Khanna et al., 2006; Rosso et al., 1995). If increases in culture counts were not observed at these temperatures, there would therefore be something inhibiting the growth of the bacteria. Nutrient-starved cells show a sharper decline in their viability at $37^{\circ} \mathrm{C}$ than at other temperatures (Nelson et al., 1996), the nutrient concentrations in the centrate, either in a nutrient-limiting or a nutrient-rich environment could therefore be a factor in the inhibition of the growth of the fecal coliforms.

\subsubsection{Test 3: Centrate characterisation}

The results from the previous test indicated that there may have been an inhibitory substance in the dewatering centrate from Treatment Plant A and that this inhibitory substance was not present in the centrate produced by running Treatment Plant A sludge in a laboratory centrifuge. Depending on the strength of the centrifuge and the nature of the inhibitory substance, the substance could accumulate in the centrate. 
From the previous tests, it was not clear what this inhibitory substance was and as such, different methods for the characterisation of the centrate would need to be undertaken. Methods to gauge the toxicity of the centrate will be discussed in Section 5.2.4 and 5.2.5. A good starting point is to do chemical characterisation of two types of centrate, one which has been shown to inhibit bacterial growth and the other which has not been shown to inhibit the growth of the bacteria. Chemicals present in the digested sludge could accumulate in high concentrations in the centrate because of their small particle size. The absence of these chemicals or nutrients in the dewatered cake samples could induce the favourable conditions for the regrowth of the bacteria or cause VBNC bacteria to exit this state.

For Test 3 of Phase II of this study, the levels of a variety of nutrients were examined and the chemical characterisation of the full-scale centrate was compared with the lab-scale centrate from the same treatment plant. As shown in Test 2, the characteristics of the centrate produced in the lab are different from those of the treatment plant and any significant difference between the concentrations of the nutrients could be responsible for the full-scale centrate showing an inhibitory effect and not the lab-scale centrate.

Table 5.2 has the results from the averages of two replicates and the standard deviations for all of the parameters tested on both sampling days, these sampling events were approximately two weeks apart but the samples were collected in the same way. Table 5.3 shows the results from the paired t-tests that were performed to compare the full-scale and the lab-scale concentrations of the parameters on each of the sampling days. On the 
first day of sampling, four parameters were significantly higher in the full-scale centrate: nitrite $(\mathrm{p}=0.013)$, reactive phosphate $(\mathrm{p}=0.019)$, sulphide $(\mathrm{p}=0.002)$ and the solids concentration $(\mathrm{p}=0.017)$. However, on the second day of sampling, only the sulphide is significantly higher $(p=0.034)$ and the total nitrogen is significantly lower in the full-scale centrate $(\mathrm{p}=0.016)$.

Table 5.2: Centrate characterisation averages and standard deviations* Samples from Treatment Plant A

\begin{tabular}{|c|c|c|c|c|}
\hline & \multicolumn{2}{|c|}{ Day 1} & \multicolumn{2}{|c|}{ Day 2} \\
\hline & $\begin{array}{c}\text { Full-scale } \\
\text { average (o) }\end{array}$ & 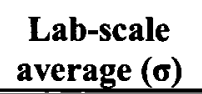 & $\begin{array}{c}\text { Full-scale } \\
\text { average }(\sigma)\end{array}$ & $\begin{array}{c}\text { Lab-scale } \\
\text { average }(\sigma)\end{array}$ \\
\hline Protein $(\mathrm{mg} / \mathrm{L})$ & $\begin{array}{c}230.9 \\
( \pm 1.57)\end{array}$ & $\begin{array}{c}217.0 \\
( \pm 11.00)\end{array}$ & $\begin{array}{c}229.5 \\
( \pm 6.68)\end{array}$ & $\begin{array}{c}260.3 \\
( \pm 10.2)\end{array}$ \\
\hline Carbohydrates $(\mathrm{mg} / \mathrm{L})$ & $\begin{array}{c}209.4 \\
( \pm 21.21) \\
\end{array}$ & $\begin{array}{c}206.5 \\
( \pm 2.26) \\
\end{array}$ & $\begin{array}{c}197.5 \\
( \pm 18.11)\end{array}$ & $\begin{array}{c}220.8 \\
( \pm 11.03)\end{array}$ \\
\hline Chemical oxygen demand $(\mathrm{mg} / \mathrm{L})$ & $\begin{array}{c}2480 \\
( \pm 42.43)\end{array}$ & $\begin{array}{c}2430 \\
( \pm 28.28)\end{array}$ & $\begin{array}{c}2475 \\
( \pm 190.92)\end{array}$ & $\begin{array}{c}2550 \\
( \pm 42.42)\end{array}$ \\
\hline Total nitrogen (mg/L) & $\begin{array}{c}1135 \\
( \pm 21.21) \\
\end{array}$ & $\begin{array}{c}1150 \\
( \pm 56.56)\end{array}$ & $\begin{array}{c}1090 \\
( \pm 0.00)\end{array}$ & $\begin{array}{c}1285 \\
( \pm 7.07) \\
\end{array}$ \\
\hline Nitrate $(\mathrm{mg} / \mathrm{L})$ & $\begin{array}{c}0.05 \\
( \pm 0.35)\end{array}$ & $\begin{array}{c}0.55 \\
( \pm 0.21)\end{array}$ & $\begin{array}{c}0.75 \\
( \pm 0.21)\end{array}$ & $\begin{array}{c}0.20 \\
( \pm 0.14)\end{array}$ \\
\hline Nitrite $(\mathrm{mg} / \mathrm{L})$ & $\begin{array}{c}1.70 \\
( \pm 0.028)\end{array}$ & $\begin{array}{c}1.35 \\
( \pm 0.014)\end{array}$ & $\begin{array}{c}1.68 \\
( \pm 0.035)\end{array}$ & $\begin{array}{c}1.51 \\
( \pm 0.014)\end{array}$ \\
\hline Ammonia nitrogen $(\mathrm{mg} / \mathrm{L})$ & $\begin{array}{c}800 \\
( \pm 14.14)\end{array}$ & $\begin{array}{c}970 \\
( \pm 113.14)\end{array}$ & $\begin{array}{c}1130 \\
( \pm 0.00)\end{array}$ & $\begin{array}{c}1700 \\
( \pm 169.71)\end{array}$ \\
\hline Reactive phosphate (mg/L) & $\begin{array}{c}320.5 \\
( \pm 2.12)\end{array}$ & $\begin{array}{c}232.5 \\
( \pm 28.28)\end{array}$ & $\begin{array}{c}280.0 \\
( \pm 0.00)\end{array}$ & $\begin{array}{c}221.0 \\
( \pm 22.63)\end{array}$ \\
\hline Sulphide $(\mu \mathrm{g} / \mathrm{L})$ & $\begin{array}{c}2720 \\
( \pm 42.43)\end{array}$ & $\begin{array}{c}1650 \\
( \pm 28.28)\end{array}$ & $\begin{array}{c}2505 \\
( \pm 63.64)\end{array}$ & $\begin{array}{c}1830 \\
( \pm 14.14)\end{array}$ \\
\hline $\mathrm{pH}$ & $\begin{array}{c}8.06 \\
( \pm 0.021)\end{array}$ & $\begin{array}{c}8.13 \\
( \pm 0.078)\end{array}$ & $\begin{array}{c}7.80 \\
( \pm 0.028)\end{array}$ & $\begin{array}{c}7.93 \\
( \pm 0.007)\end{array}$ \\
\hline Solids concentration (\%) & $\begin{array}{c}0.256 \\
( \pm 0.001)\end{array}$ & $\begin{array}{c}0.232 \\
( \pm 0.000)\end{array}$ & $\begin{array}{c}0.266 \\
( \pm 0.008) \\
\end{array}$ & $\begin{array}{c}0.288 \\
( \pm 0.000)\end{array}$ \\
\hline Volatile Acids (mg/L) & $\begin{array}{c}340.0 \\
( \pm 15.56)\end{array}$ & $\begin{array}{c}309.5 \\
( \pm 23.33)\end{array}$ & $\begin{array}{c}327.0 \\
( \pm 16.97)\end{array}$ & $\begin{array}{c}376.5 \\
( \pm 3.53)\end{array}$ \\
\hline Total organic carbon (mg/L) & $\begin{array}{c}1200 \\
( \pm 84.85)\end{array}$ & $\begin{array}{c}1210 \\
( \pm 70.71)\end{array}$ & $\begin{array}{c}958 \\
( \pm 0.00)\end{array}$ & $\begin{array}{c}1107 \\
( \pm 0.00)\end{array}$ \\
\hline
\end{tabular}

*Averages are for two replicates 
Table 5.3: Two tailed t-test for the comparison for average parameter concentrations

\begin{tabular}{|r|c|c|}
\hline & Sig. (2-tailed) & Sig. (2-tailed) \\
\hline Protein & 0.320 & 0.087 \\
Carbohydrates & 0.880 & 0.286 \\
COD & 0.316 & 0.677 \\
Total N & 0.775 & $0.016^{*}$ \\
Nitrate & 0.255 & 0.110 \\
Nitrite & $0.013^{*}$ & 0.064 \\
Ammonia-N & 0.276 & 0.132 \\
Reactive Phosphate & $0.019^{*}$ & 0.169 \\
Sulfide & $0.002^{*}$ & $0.034^{*}$ \\
pH & 0.414 & 0.085 \\
Solids (\%) & $0.017^{*}$ & 0.150 \\
Volatile Acids & 0.281 & 0.056 \\
TOC & 0.910 & NA \\
\hline *samples are significantly different ( 0.05$)$
\end{tabular}

Sulphide can be generated by sulfate-reducing bacteria in fecal samples or other fecal bacteria (Ohge et al., 2005). It's presence in samples is a concern because it is toxic and corrosive (Londry and Suflita, 1999). Work has shown that high concentrations of sulphide are toxic in anaerobic digesters (Rudolfs and Amberg, 1952), the same could be true for samples after their exit from the anaerobic digester. A study in 1997 showed that in the presence of light and sulphide, fecal coliforms experience a drop in culturable cells but not a drop in viable cells (Dan et al., 1997). The sulphide concentrations in the fullscale centrate were significantly higher than those from the laboratory. If the presence of this centrate led to the entry of the fecal coliforms into the VBNC state, once the centrate was removed, it would be possible that the cells could exit this state and that a rise in 
fecal coliforms could be observed. As this chemical characterisation was by no means complete, it could be that another substance is inhibiting the growth, such as the accumulation of heavy metals in the centrate, for which previous research has shown to occur (El-Aassar et al., 1998).

Some studies have looked at the correlation between the survival of fecal coliforms and the concentration of other nutrients. Reactive phosphate or orthophosphate, is said to lead to an increase in fecal coliforms (Aslan-Yilmaz et al., 2004). It was also found that orthophosphate was weakly but significantly correlated with fecal coliform abundance (Mallin et al., 2000). Very little information can be found on the effect of nitrite on fecal coliform counts, most papers focus on nitrogen, ammonia and nitrate rather than nitrite, for example: (Field et al., 2007).

\subsubsection{Test 4: Centrate seeding}

After doing a basic chemical analysis, different options were examined for a more indepth study on the toxicity of the different types of centrate. Most toxicity methods rely on the survival of an indicator species to measure how toxic the sample is (Ren and Frymier, 2004). A common method is the Microtox assay (Azur Environmental) which has been used for the toxicity of municipal waste activated sludge contaminated with industrial compounds (Ricco et al., 2004). This assay uses luminescent bacteria and the increase or decrease in the light output from the species as a measure of the toxicity (Azur Environmental, 1998). However, the toxicity in the centrate, both full-scale and lab-scale may not have been high enough to be detected by the assay. Previous research 
has shown it to not be sensitive enough to detect any acute toxicity in wastewater samples (Arbuckle and Alleman, 1992), the same may be true for centrate samples. Another method to observe the toxicity of a sample is to examine the growth and decline of a seeded culture into the sample. These experiments have been shown to be useful in determining inactivation of bacteria but do not always represent the naturally-occurring organisms in the samples (Tree et al., 2003). For this reason, it was decided that seeding the sterilised centrate with bacteria which were indigenous to the sludge would be ideal.

Two different centrate seeding sample runs were undertaken; the first one using an indigenous culture and the second using a purchased (ATCC) strain of $E$ coli being seeded into the sample. A sample that was more toxic would see a sharper decrease in the seeded culture as the environment was less favourable for its growth or survival. Total coliform bacteria, isolated from a sludge sample, were seeded into sterilised samples of lab and full-scale centrate. However, the samples were overgrown with fecal coliforms and even the most diluted samples had all of their test tubes being positive for fecal coliform presence. No conclusions could be drawn from the results (Data not shown).

There were a few issues with the first run of seeding testing, i.e. that the sample was overgrown and that total coliforms were used which would not necessarily represent the fecal coliform category. To counteract these, a new test was done, this time using a single known strain of fecal coliform ( $E$ coli ATCC \#11229). This strain was extracted from a Kwik Stik tube and spread onto a non-selective media plate. The difference with this test is that when extracting the colonies and placing them into the centrate, no broth was 
added to the sterilised centrate. This broth provides nutrients for the growth of the bacteria and could modify the possible inhibitory effect of the centrate. For the second experiment, the colonies were removed with an inoculating loop and mixed into the sterilised centrate without transferring any of the broth. Three colonies were taken for each of the samples.

The samples of centrate were compared with phosphate-buffered solution in order to examine the survival of the seeded $E$ coli alone, without any of the components from centrate. It was important to compare the levels in the centrate with a nutrient-limiting environment in order to rule out that any decreases observed were caused simply by the bacteria in the centrate starving, if the decreases were more prominent than those in a nutrient limiting environment they may have been caused by the toxicity of the sample.

The results from the single strain seeding can be found in Figure 5.16. For this test, because of the low solids content of the samples, the y axis is given in terms of grams wet weight instead of $\mathrm{g}$ DW. The reason being that dividing the MPN value by the solids concentration to get the MPN in terms of $\mathrm{g}$ DW would raise the level of fecal coliforms; for an undiluted sample which showed no growth, the MPN/g DW was log 3.8 .

Figure 5.16 is split into three windows for the different temperatures for the test, a) refrigerator, b) room temperature and c) incubator. On the graph, the arrows signify that the fecal coliform levels are either higher or lower than the value given, meaning that either all of the samples or none of the samples showed gas production at all of the 
dilutions studied. The initial counts are given as larger than $\log 4.2$ for both of the centrate samples and as larger than log5.2 for the PBS samples. All of the samples were split up for aging at the different temperatures after the initial enumeration which is why the coliform levels for all of the temperatures are the same. The initial counts for all of the samples could not be obtained as all of the A-1 tubes were positive for gas production even at the highest dilution. On the figure, it appears that the counts of the full-scale samples increase more than the lab-scale samples, however, as the actual fecal coliform level can be anywhere above the point on the graph, it could be that the initial bacterial count for the full-scale centrate was higher than the lab-scale centrate.

Despite not having the exact initial count it is still possible to look at the increase and decrease or the fate of the fecal coliforms for each sample by the slope of their decline. The full-scale samples in the refrigerator (Figure 15.6a) have a steeper slope and thus a sharper decrease in fecal coliforms than the lab-scale samples. In a nutrient-limiting environment (the PBS sample), the fecal coliforms decrease right away and at day two, the samples showed no fecal coliform presence even for an undiluted sample shown as a level of zero on the figure and without error bars or arrows. In the refrigerator, the fecal coliforms are under so much stress as well as being in a state of starvation and as such all become nonculturable by day two. 

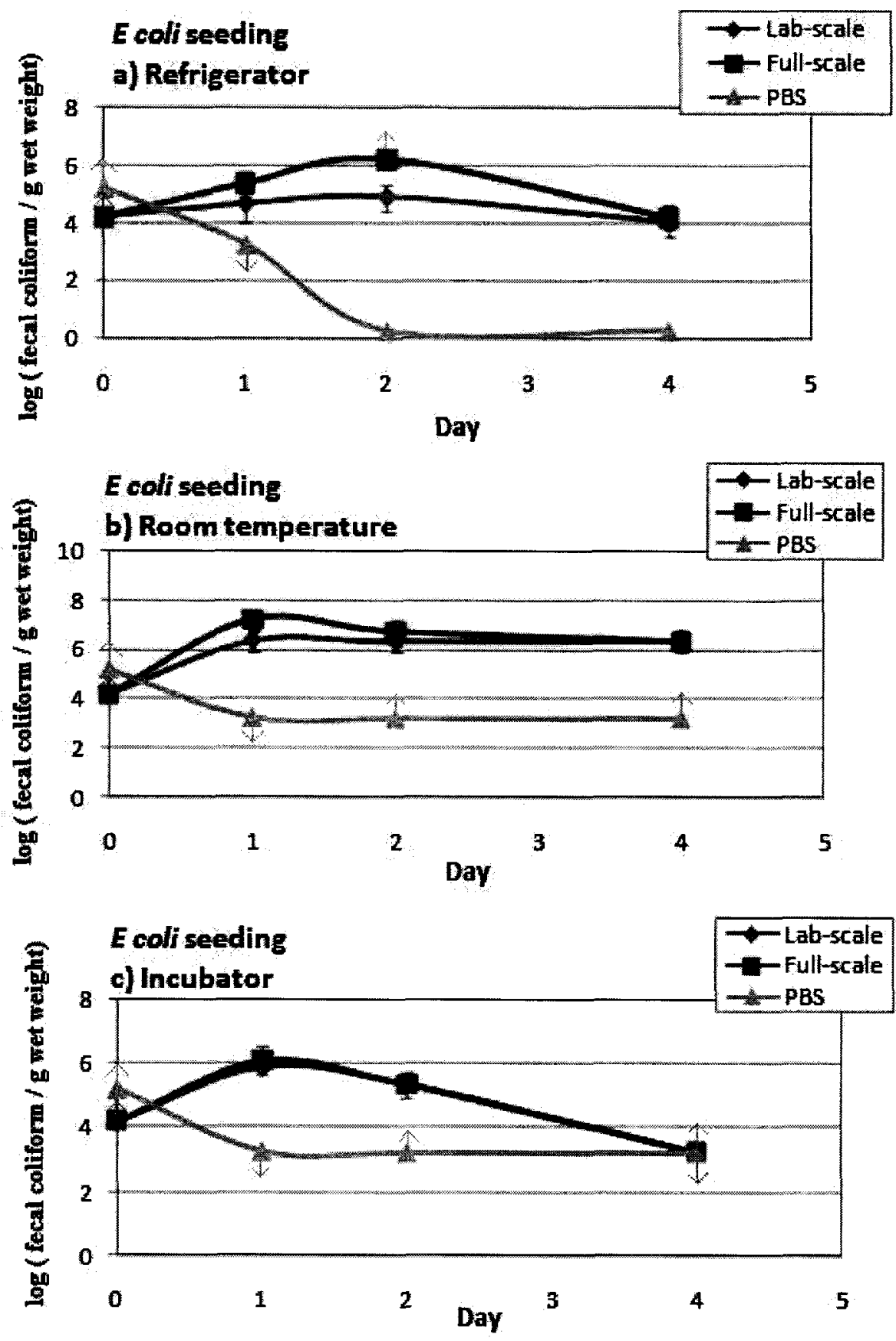

Figure 5.16: Fecal coliform levels for lab-scale and full-scale centrate samples from Treatment Plant A and PBS seeded with $E$ coli ATCC 11229 . Refrigerator $=4^{\circ} \mathrm{C} \pm 0.5^{\circ} \mathrm{C}$, room temperature $=24^{\circ} \mathrm{C} \pm 1{ }^{\circ} \mathrm{C}$, incubator $=35^{\circ} \mathrm{C} \pm 0.5^{\circ} \mathrm{C}$. Detection and enumeration done by A-1 medium. 
Because the initial counts of the full-scale and lab-scale centrate are not known, the main difference which can be compared between these two sample types is the slope on the survival graph. A steeper slope might be interpreted as a more stressful environment as the decline in fecal coliforms is more important. For $E$ coli in the refrigerator from day two to day four, the lab sample had a slope of -0.49 whereas the full-scale sample had a slope of -0.94 . Since the full-scale sample was more negative, the environment would be considered more stressful.

At room temperature (Figure 5.16b), there are also higher levels of fecal coliforms in the full-scale samples, a higher decrease is also observed in the full-scale sample as opposed to the lab-scale sample. In a nutrient-limiting environment, the fecal coliforms drop initially but then level off, showing positive test tubes for fecal coliforms even at $10^{-3}$ dilution which is only slightly lower than the samples of centrate which stay steady but then decline steeply after two days. The slope of the survival of fecal coliforms from day one to day four was -0.27 for the full-scale sample and 0.02 for the lab-scale sample. For room temperature as well as in the refrigerator, the decline in fecal coliforms is more dramatic for the full-scale rather than the lab-scale sample.

In the incubator (Figure 5.16c), full-scale and lab-scale centrate samples show a similar trend with an increase followed by a dramatic decrease by day four. What is important to note on this graph is that the levels in the centrate decrease to a point that they are slightly lower than the nutrient-limiting PBS sample. As well, the arrows on the figure show that the cake + PBS fecal coliform levels are higher than the point on the figure and that the 
levels for the centrate samples are lower than their respective points in the figure. The PBS sample had the same trend in the incubator as at room temperature, after an initial decrease from the organisms that were too stressed to retain their culturability, the levels stayed steady. Both centrate types decreased to lower levels and it can be assumed that it cannot be due simply to starvation as a starved environment could sustain a larger population than the ones in the sterilised centrate. The data points to the hypothesis that there is an inhibitory substance in the centrate which prevents the growth of bacteria. This substance has the largest effect in the incubator. As the declines were more prominent for the full-scale samples, it could be assumed that the environment was stressful for the bacteria and perhaps toxic, however, more tests are needed to verify this hypothesis.

\subsubsection{Test 5: Cellular ATP}

Another way to get an idea of the toxicity of the sample is to measure the ATP concentrations in the samples. Intracellular ATP content will drop in the presence of a toxic substance as some cells will die (Dalzell and Christofi, 2002). The ATP measurements are said to be more sensitive to lower toxicity samples than Microtox (Arretxe et al., 1997), which is an advantage for the project at hand as the toxicity is not expected to be high and because what is most important is simply to compare the toxicity of the two types of centrate. On top of giving us information about the toxicity of the substances, the intracellular and extracellular contents of ATP can also give information about the relative abundance of viable cells in the samples (Nicolau et al., 2004), this 
information can be compared with the culturable bacteria counts in order to see the changes in the viable/culturable bacteria with aging at different temperatures.

\subsubsection{Toxicity of centrate samples}

The toxicity of the samples is measured with the Biological Stress Index (BSI \%), calculated by dividing the extracellular ATP by the total ATP (extracellular + intracellular ATP). This method has been used successfully for bioreactors (Bergeron and Paice, 2001) and the same method was used for this experiment. The solid concentrations of bioreactors and centrate are similar and as such no modifications needed to be made for the method's use for centrate. In a bioreactor, a BSI between $30 \%$ and $50 \%$ would require preventative action and a $\mathrm{BSI}$ above $50 \%$ would require corrective action (LuminUltra Technologies, 2007). This would mean that the environment for the bacteria would be considered stressful. Preliminary tests on lab-scale and full-scale centrate gave BSIs of $20.4 \%$ and $37.6 \%$, respectively. Since the BSI for the full-scale centrate is higher, it can be said that for this sampling event, it is a more stressful environment or that there is a higher proportion of extracellular ATP or dead cells in the sample than in the labscale sample.

In order to test if there were any changes in the stress index for the bacteria throughout the sludge treatment train of Treatment Plant A, ATP concentrations were calculated for all the locations outlined in Figure 4.1. Figure 5.17 has the results from this test. In the full-scale studies, there was no significant change in fecal coliform numbers throughout the treatment train, however, even though the amount of culturable bacteria did not 
change, the stress that those bacteria are placed under did change. The sludge coming out of the digester has a high biological stress index at $48 \%$. After the addition of the polymer, the BSI drops down to $25 \%$. After full-scale centrifugation, the solids portion has a low BSI of $15 \%$ whereas the centrate has the highest BSI at $63 \%$. This indicates that a larger ratio of extracellular ATP is found in the centrate than in the sludge cake, or perhaps that the extracellular ATP accumulates in the centrate following centrifugation. As the samples go through the treatment train, the stress levels decrease towards the dewatering. After dewatering, the cake has a very low biological stress index whereas the centrate has a high BSI (\%).

BSI (\%) Treatment Plant A

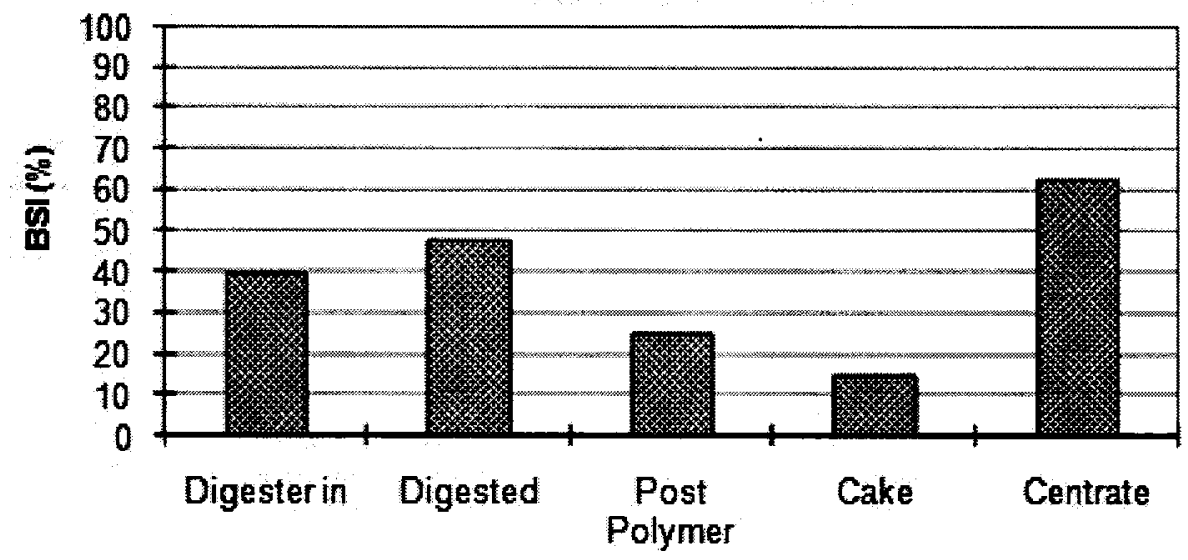

Figure 5.17: Biological Stress Index (\%) for samples taken throughout the treatment train at plant A. LuminUltra method used for all samples

BSI (\%) of lab-scale and full-scale centrate samples were compared in Figure 5.18. The lab-scale centrate had a BSI of 53\% compared with the BSI in the full-scale centrate of $63 \%$. In the same way as the full-scale centrate, the lab-scale centrate could accumulate the extracellular ATP in it post-dewatering, instead of it ending up in the cake. The cells may get damaged during centrifugation and the damaged cells with extra-cellular ATP 
could be concentrated in the centrate portion. The results from the first Luminase test correspond with the aging tests of Treatment Plant $\mathrm{A}$ at room temperature and in the incubator (Figures $5.11 \mathrm{~b}$ and $\mathrm{c}$ and $5.15 \mathrm{~b}$ and $\mathrm{c}$ ). The samples of centrate and of digested sludge see a decrease in culturable bacteria with aging at these optimum growth temperatures. If the environment for the cells is stressful, the counts would eventually drop down. That is what is seen with the aging tests as well as with the Luminase data. After some time in this stressful environment, the survival of the bacteria would be expected to be affected as shown through a drop in culturable bacteria.

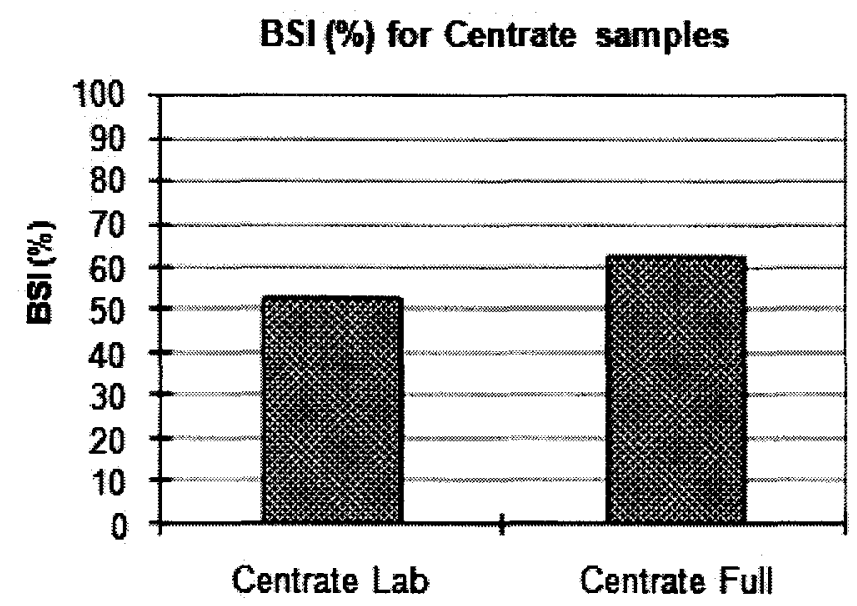

Figure 5.18: Biological Stress Index (\%) for samples of full-scale and lab-scale centrate from Treatment Plant A. LuminUltra method used for all samples

The Biological Stress Indexes for the full-scale and the lab-scale centrate samples were calculated for three different temperatures during a 30 day period. Figure 5.19a has the results for the full-scale samples and Figure $5.19 \mathrm{~b}$ has the results for the lab-scale samples. There is a lot of variability in these samples and no replicates could be undertaken as there was only a limited quantity of reagent and as the test was costly. The 
lack of replicates may account for the variations in the samples. However, it is possible to observe trends for the samples. In general, when the temperature increases, the biological stress index decreases. The other trend that can be observed is that BSIs tend to decrease with time, this is observed at all three of the temperatures.

The full-scale samples consistently have slightly higher BSI (\%) than those from the labscale. The averages of all days for the refrigerator samples are $44.4 \%$ for the lab-scale and $50.5 \%$ for the full-scale. At room temperature, the lab-scale samples had an average BSI of $43.1 \%$ and the full-scale centrate had a BSI of $46.6 \%$. In the incubator, the BSI for the lab-scale centrate samples was $39.7 \%$ and for the full-scale samples, it was $41.5 \%$. These results, as well as the centrate seeding results point to the full-scale centrate being more toxic than lab-scale centrate and are congruent with the hypothesis than an inhibitory substance can exist in the centrate. 

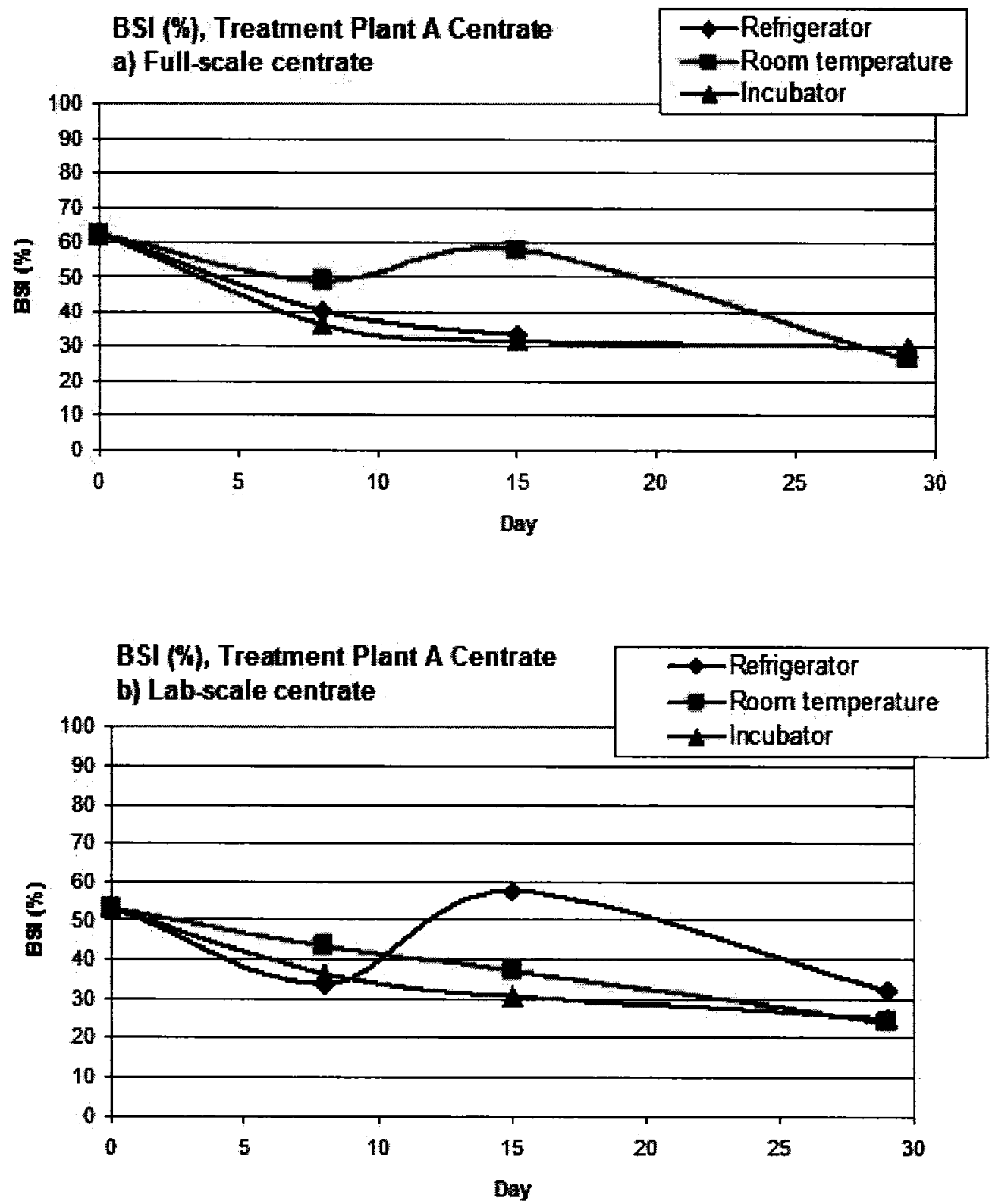

Figure 5.19: Biological Stress Index (\%) for centrate samples from Treatment Plant A placed in the refrigerator $=4^{\circ} \mathrm{C} \pm 0.5^{\circ} \mathrm{C}$, at room temperature $=24^{\circ} \mathrm{C} \pm 1^{\circ} \mathrm{C}$ and in the incubator $=35^{\circ} \mathrm{C} \pm 0.5^{\circ} \mathrm{C}$. LuminUltra method used for all samples 


\subsubsection{Total bacteria counts vs culturable fecal coliforms}

With the same data as for the BSI, it is possible to gauge the viable cell population and compare this data with the culture counts. The Luminase method for the viable cell counts using ATP was developed for bioreactors as well, and has been shown to be successful in calculating the active cell biomass just as the MLSS (Mixed Liquor Suspended Solids) concentrations which is typically used for active biomass calculations (LuminUltra Technologies, 2004). The Luminase method works on the principle that bacteria will maintain their regular intracellular ATP levels if they are viable, even if they have lost their culturability (Oliver, 1993). The viable cell counts were determined throughout the treatment train and compared with culture counts calculated with the MPN method, Figure 5.20. The higher the intracellular ATP values, the more total viable cells there are and the higher the extracellular ATP concentration, the more dead cells there are in the sample. As expected, the influent to the digester, which is made up of primary sludge and thickened waste activated sludge had high levels for both intracellular ATP and culturable fecal coliforms (MPN). This means that the amount of total viable cells is high and that the culturable fecal coliform concentrations are also high. After exiting the digester however, the total cells drop much more than the fecal coliforms and continue to drop. After going through the centrifuge, a higher concentration of both intracellular and extracellular ATP is found in the centrate sample. This figure shows that fecal coliforms behave differently than the total cell population as the culture counts show a different trend throughout the treatment train than the total cell population does. 
ATP vs MPN Treatment Plant A

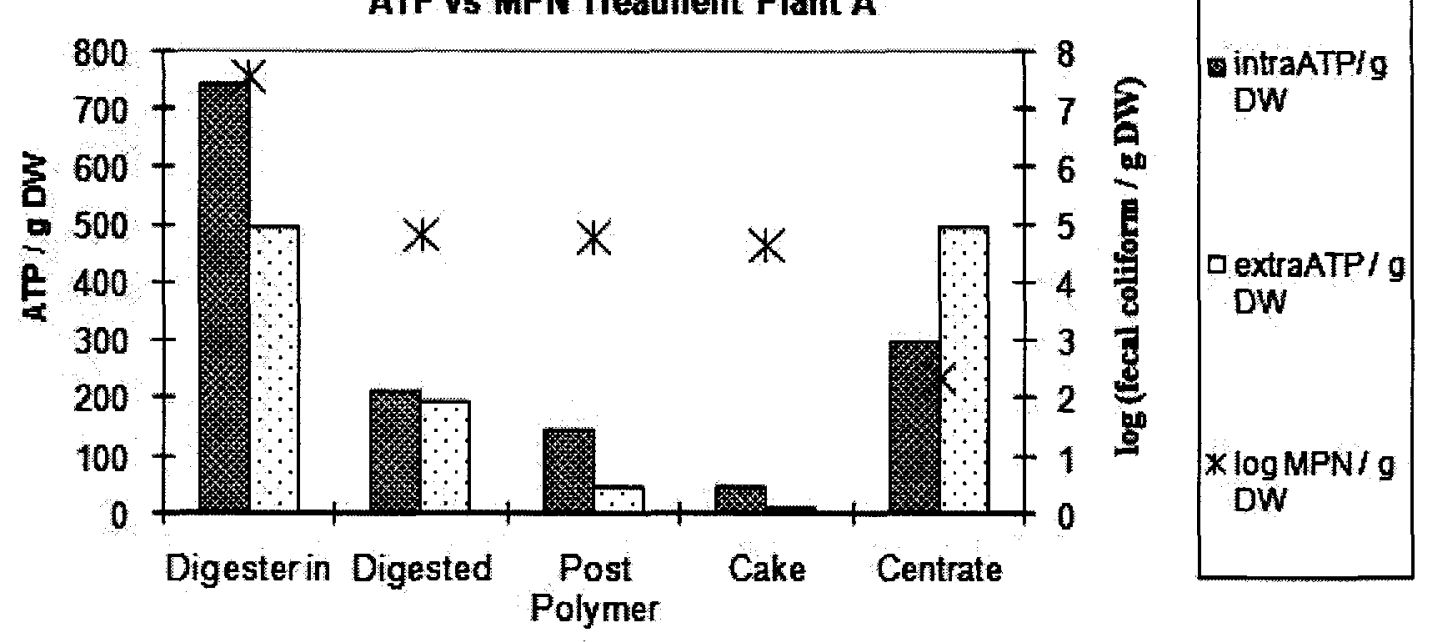

Figure 5.20: ATP/g DW value and log MPN/g DW values for samples throughout the treatment train. LuminUltra method used for all ATP sampling and MPN detection and enumeration done by A-1 medium.

${ }^{*}$ MPN values for Digester In, Digested, Post Polymer and Cake were taken from a previous sampling event.

ATP vs MPN concentrations for the centrate samples are shown in Figure 5.21, culturable fecal coliform results are given in terms of $\log$ MPN / $g$ wet weight and ATP concentrations are given in $\mathrm{ng} / \mathrm{ml}$ as the samples are only compared with other centrate samples. Although the MPN values for the full-scale centrate are higher, the intracellular ATP concentrations are lower than the lab-scale centrate. Lab-scale centrate has a higher total viable cell population but less of that population is culturable fecal coliforms. 


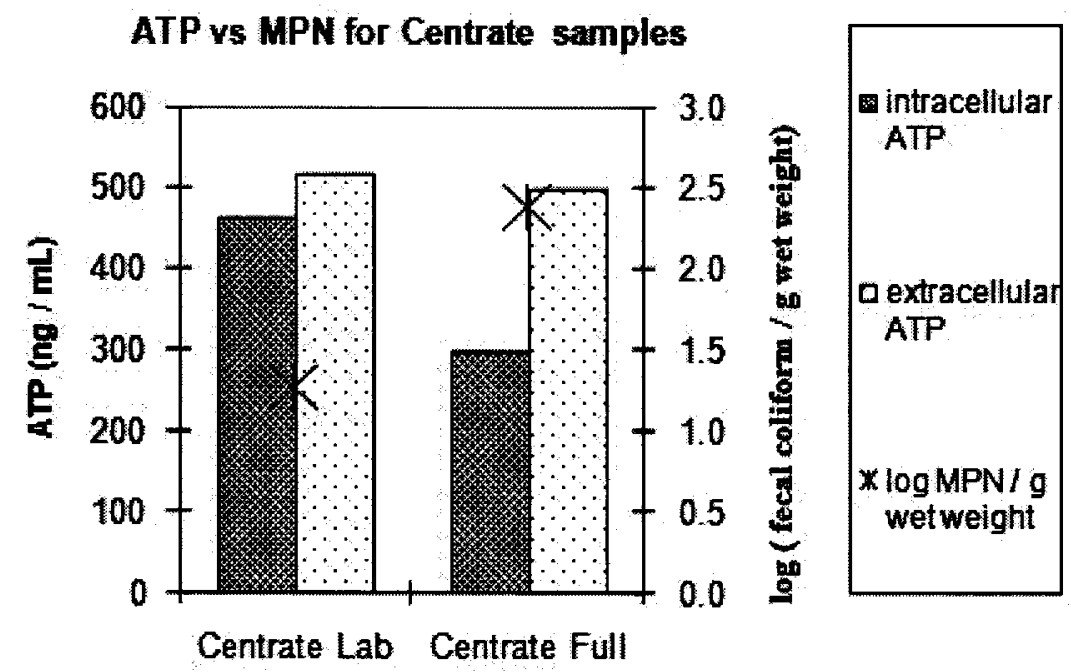

Figure 5.21: ATP/g DW values on primary y axis and $\log$ fecal coliform/ $\mathrm{g}$ DW on secondary $\mathrm{y}$ axis for samples of full-scale and lab-scale centrate. LuminUltra method used for all ATP sampling and fecal coliform detection and enumeration done by A-1 medium with MPN.

ATP results can be compared with the MPN values through a long aging time at the three different temperatures for cake, full-scale centrate and lab-scale centrate. For the following three figures, results are given in terms of wet weight as all samples have comparable solid concentrations. The cake sample ATP concentrations are given in Figure 5.22a and MPN concentrations are given in Figure 5.22b. If any error had been introduced because of the methodology with the cake samples, it would be similar for all of these samples and they could still be compared with each other quite easily. Another important point is that the Luminase method measures the ATP from all cells, not just those from fecal coliforms, and so if the ATP concentrations go up, it could be due to other microorganisms in the sample proliferating instead of the fecal coliforms. 

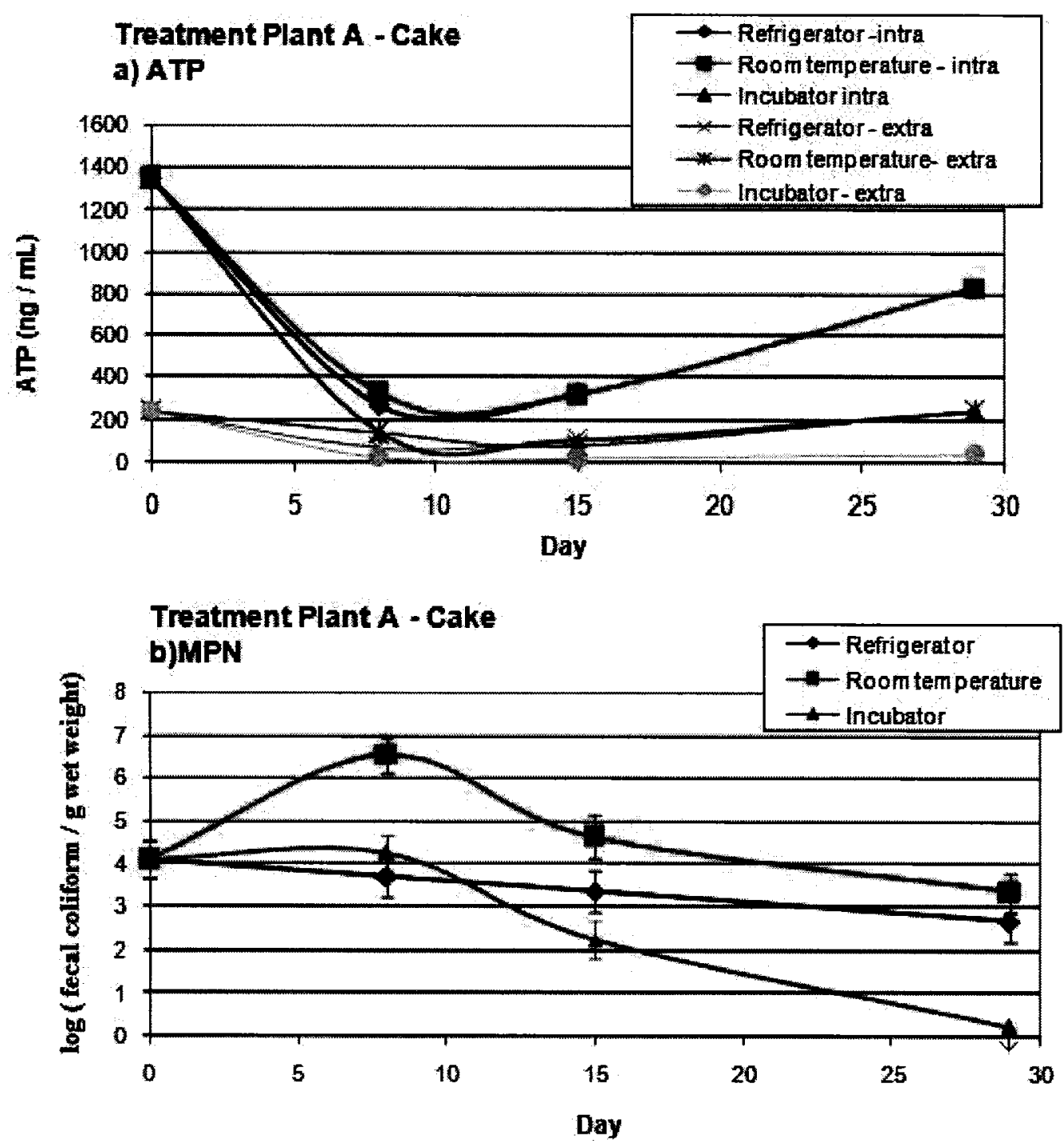

Figure 5.22: Fecal coliform and ATP values for cake samples aged at three temperatures: Refrigerator $=4^{\circ} \mathrm{C} \pm 0.5^{\circ} \mathrm{C}$, Room temperature $=24^{\circ} \mathrm{C} \pm 1^{\circ} \mathrm{C}$ and Incubator $=35^{\circ} \mathrm{C} \pm 0.5^{\circ} \mathrm{C}$. Fecal coliform detection and enumeration done by A-1 medium with MPN. LuminUltra method used for all ATP sampling and enumeration.

On Figure 5.22a, viable bacteria, given by the intracellular ATP, at all temperatures have a similar trend with a drop and then a rise after day eight. The incubator has the lowest values for intracellular and extracellular ATP. Looking at Figure 5.22b, the MPN values are also the lowest in the incubator, after an initial rise that is. The rise in total cells, 
observed as intracellular ATP, is not due to fecal coliforms as there is no growth by Day 20. In the refrigerator, the MPN values do not change much during the 30 day testing period, as for the room temperature samples, they show a large increase and then drop back down to their initial levels by Day 30. As in the incubator, the rise in intracellular ATP is not likely to be caused by fecal bacteria as the MPN values either stayed steady or dropped slightly. In the incubator the culturable fecal coliform rise in the first two to four days but then drop down lower than the original counts. This shows that with time the environment in the incubator becomes inhospitable for the cake.

Lab-scale centrate ATP data is given in Figure 5.23a and the MPN for the 30 days of testing is given in Figure 5.23b. For the samples stored at room temperature and in the incubator, the culturable fecal coliform values drop to undetectable levels after eight days in the incubator, shown as zero culturable fecal coliforms on the figure. The pattern for both the total cells and the fecal coliforms is the same with a drop and then remaining steady at a low value. At room temperature, the intracellular ATP does go up at day 15, this pattern is not seen for the culturable fecal coliform values, after day 15 , there are no culturable fecal coliforms left in the sample. As in the cake samples, this rise in total cells is likely due to non-fecal bacteria. In the samples stored in the refrigerator, the ATP and the MPN have the same pattern with a slight drop after 30 days, but no dramatic increases or decreases throughout the sampling time. 

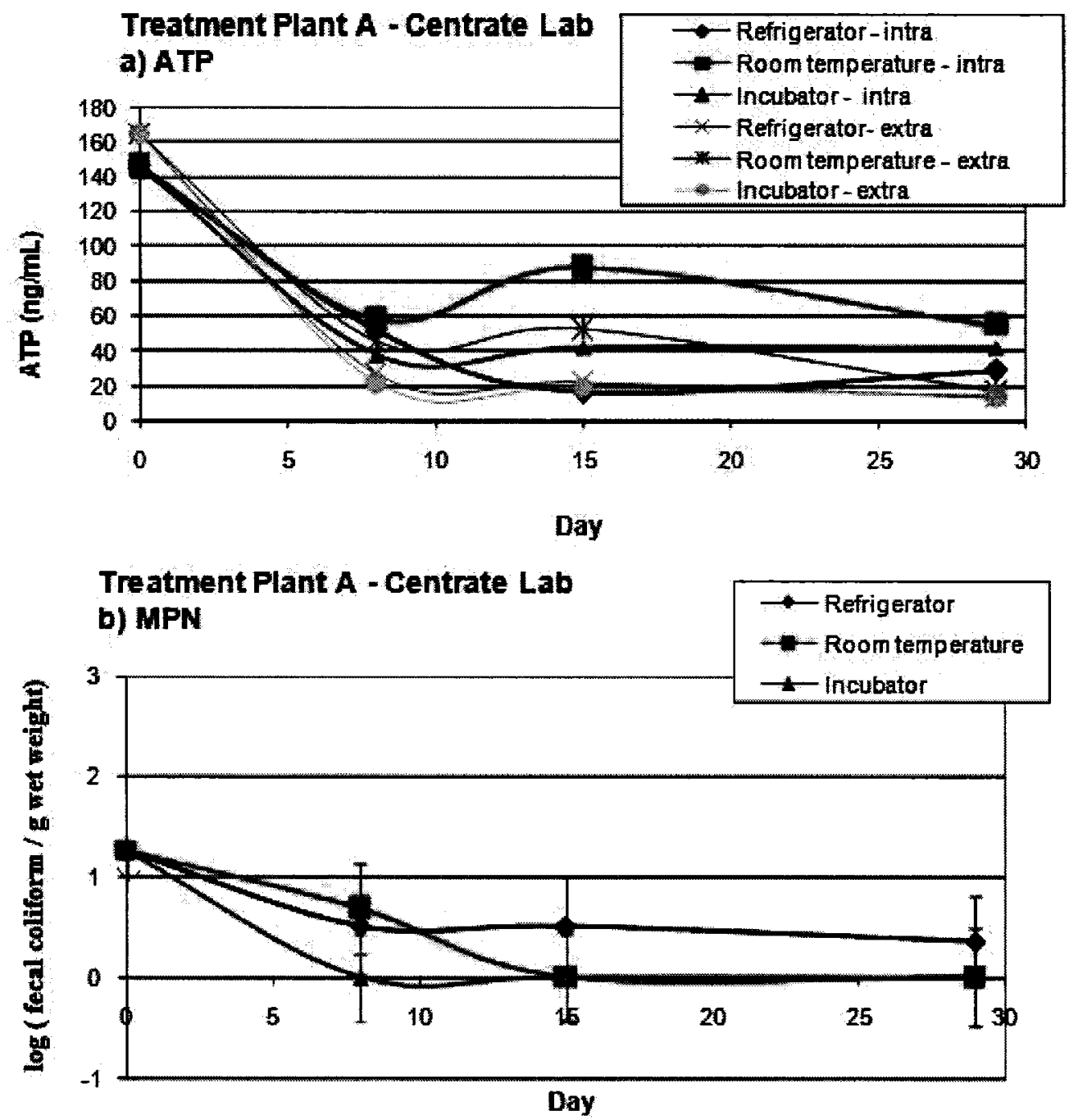

Figure 5.23: Fecal coliform and ATP values for lab-scale centrate samples aged at three temperatures: refrigerator $=4^{\circ} \mathrm{C} \pm 0.5^{\circ} \mathrm{C}$, Room temperature $=24^{\circ} \mathrm{C} \pm 1^{\circ} \mathrm{C}$ and Incubator $=$ $35^{\circ} \mathrm{C} \pm 0.5^{\circ} \mathrm{C}$. Fecal coliform detection and enumeration done by A-1 medium with MPN. LuminUltra method used for all ATP sampling and enumeration.

The full-scale centrate ATP data is given in Figure 5.24a and the MPN data is given in Figure 5.24b. For these samples, the MPN values started off higher than for the labcentrate samples. In the incubator, the culturable fecal coliforms drop to undetectable levels after day eight but in the case of the full-scale centrate at the room temperature, the 
culturable fecal coliform values do not drop until day 30 . Although the culturable fecal coliforms drop dramatically in the incubator, the total cell counts stay high, with the highest count for either of the centrate types, throughout the sampling period of $89 \mathrm{ng} /$ $\mathrm{ml}$. This highest total cell count is in the incubator, where the culturable fecal coliforms show no additional growth. The increase in total cells could be due to competing bacteria, perhaps it is the competition from a different type of bacteria which leads to the unfavourable or stressful conditions for the fecal coliforms in the sample. It could also be that the stressful conditions lead to a decrease in culturable fecal coliforms and because there are less fecal bacteria, other microorganisms can take over the niche usually taken by them.

Since this method has not been reported to be used for sludge cake or centrate samples, there is very little literature about the changes in ATP in sludge samples. Research that does exist on the subject typically measures only intracellular ATP as this is the parameter which gives the total cell population. ATP has been used successfully as a measure of biological activity during the composting of sewage sludge samples, the temperatures in this case being $40-70^{\circ} \mathrm{C}$. After 11 months of composting, the levels of ATP in land-farmed oil-refinery sludge showed an increase and then a drop back down to its original levels (Marin et al., 2005). 

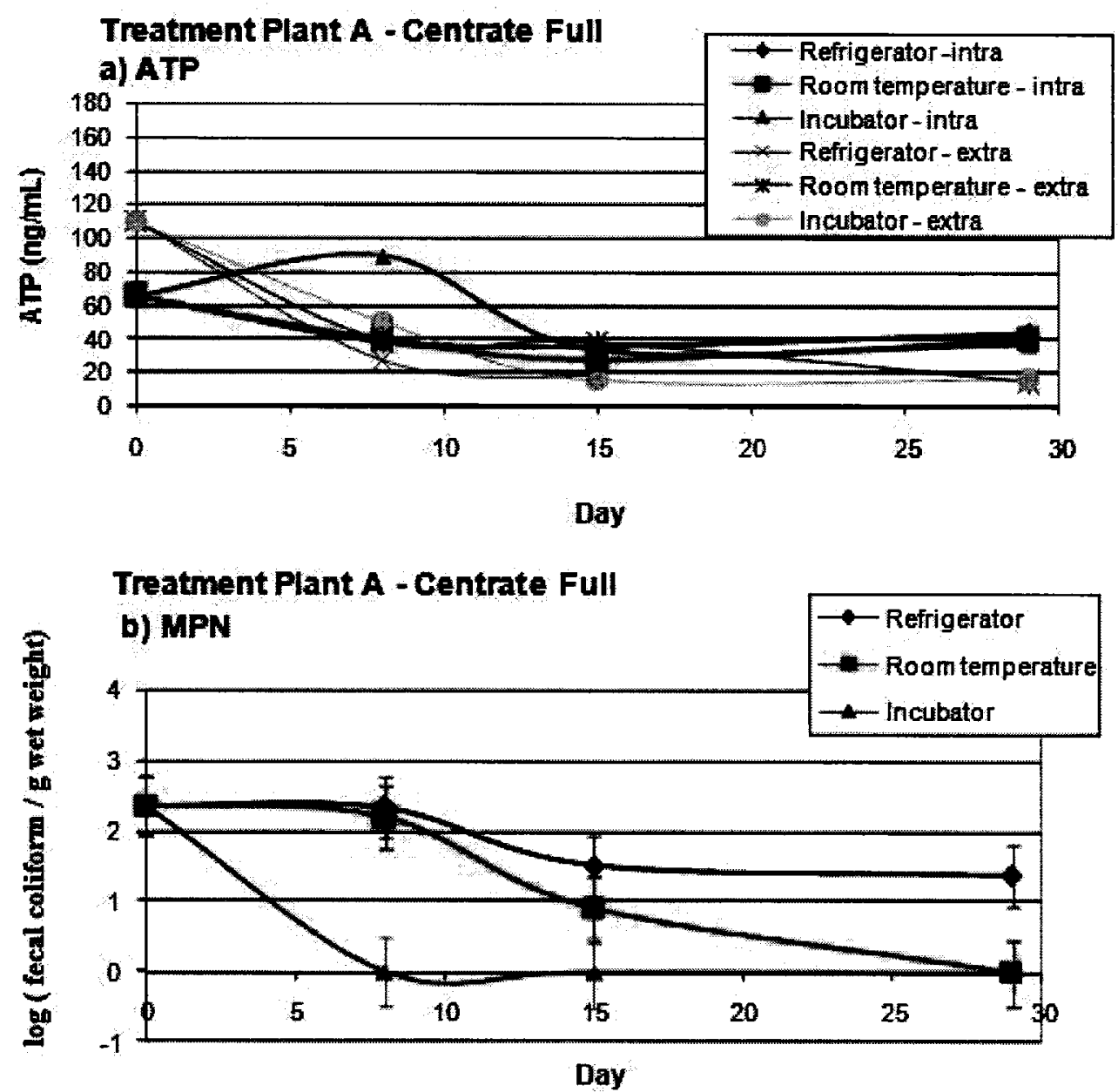

Figure 5.24 Fecal coliform and ATP values for full-scale centrate samples aged at three temperatures: refrigerator $=4^{\circ} \mathrm{C} \pm 0.5^{\circ} \mathrm{C}$, Room temperature $=24^{\circ} \mathrm{C} \pm 1^{\circ} \mathrm{C}$ and Incubator $=$ $35^{\circ} \mathrm{C} \pm 0.5^{\circ} \mathrm{C}$. Fecal coliform detection and enumeration done by A-1 medium with MPN. LuminUltra method used for all ATP sampling and enumeration.

The effect of different environmental stresses was looked at by another paper. The range of temperature for the study was $10-44^{\circ} \mathrm{C}$, the intracellular ATP increased slightly and reached a peak at $35^{\circ} \mathrm{C}$ and then dropped down as the temperature increased more. These results were from one hour of incubation but showed that the optimum temperature for total cell counts was incubation at $35^{\circ} \mathrm{C}$ (Brezonik and Patterson, 1971). Higher total cell 
counts were observed in the current study in the incubator, even when the fecal coliform counts decreased. Also, the BSI (\%) were lower in the incubator, indicating that the conditions were less stressful. Another study on amended sewage sludge showed a decrease in intracellular ATP as the incubation time went on for a total of eleven weeks. The temperature for the latter study was kept at $28^{\circ} \mathrm{C}$ (Diazburgos et al., 1993). At this temperature, the behaviour of the samples would be closest to the room temperature samples of this study. However, for all samples in the current study, an increase was seen after 4 weeks at room temperature, there must be some type of bacteria which is able to grow under the conditions of this study; these would not be fecal coliforms.

The ATP method has also been used on cultures of $E$ coli to test their loss of culturability (Roth et al., 1988). Even after being exposed to a saline stress, the cells continued to accumulate intracellular ATP whereas their culturable counts dropped down within two hours of the stress being placed on the cells. Had any of the cells become nonviable, extracellular ATP would increase. In their study, no extracellular ATP was found (Roth et al., 1988). What should be kept in mind for this test is that a pure culture was used in a synthetic setting; the fecal coliforms in a sludge environment are affected by many other aspects of their environment and may therefore not behave in the same way as the pure culture.

The Luminase ATP method is quite innovative and its use for centrate and cake samples could continue with further research in the coming years. The issues with the extraction of the ATP from cake samples have already been described, further work could be done 
in order to minimise the possible error introduced by the use of the method for solid samples. The method was very successful for use with centrate samples, as their solids concentrations were lower and their concentrations of intracellular and extracellular ATP were well within the range of detection. The success of this method is especially true with regards to the BSI (\%). More replicates would have led to a higher degree of significance between the two types of centrate, however, with the expense of the reagent and the time required for the sampling, this was not possible. The main trend did remain though, that the full-scale centrate samples had higher BSI (\%) than lab-scale samples. 


\section{Chapter 6}

\section{Conclusions}

The main conclusions for the study will be split by the two different phases and will be presented in point form.

\subsection{Phase I}

Testing for Phase I used a laboratory centrifuge, therefore the following conclusions are given for the lab-scale tests and as such, may not be applicable to the full-scale dewatering centrifuges.

- Changes in the speed of the laboratory centrifuge, representing different levels of shear, do not appear to cause increases in the fecal coliform counts through centrifuge dewatering.

- Polymer dose, coupled with different levels of shear, does not significantly affect the fecal coliform counts. The centrifuge did not cause increases from the breaking up of the colonies of fecal coliforms from the polymer being ripped off the sludge.

- The polymer type: its physical form, molecular weight and cationic charge as well as its dose, does not appear to affect the coliform count. 


\subsection{Phase II}

The results from Phase II were obtained from full-scale samples as well as compared with samples obtained from the laboratory centrifuge.

- The reactivation / regrowth phenomenon is not observed at all treatment plants that employ anaerobic digestion and dewatering by centrifugation.

- Fecal coliform counts in cake samples from Treatment Plant A exhibited a significant increase at room temperature but a decrease in the refrigerator.

- Fecal coliform counts in cake samples as well as pellet samples from Treatment Plant B do not exhibit significant increases or decreases if they are left at room temperature or in the refrigerator.

- The results from the full-scale experiments at Treatment Plant A indicate that regrowth is observed in the absence of centrate. This indicates the presence of an inhibitory substance in the centrate.

- The characteristics of the sludge cake and centrate from Treatment Plant A and Treatment Plant B are different as the inhibitory effect from the centrate is not observed to such an extent at Treatment Plant B. 
- The characteristics of the cake and centrate of lab-scale samples are different than those obtained from the treatment plant itself, even if the digested sludge samples are from the same plant. No significant growth inhibition was observed for any of the lab-scale samples. The lack of reliable simulation of full-scale centrifuges is a significant limitation to the current research on the topic.

- Chemical characterisation of the centrate identified sulphide as a possible growth inhibitor as its concentration was significantly higher in full-scale centrate than labscale centrate.

- According to centrate seeding as well as intracellular versus extracellular ATP concentrations, the full-scale centrate appears to provide a more stressful environment for the bacteria than the lab-scale centrate.

- The incubator (at $35^{\circ} \mathrm{C}$ ), provides the optimum temperature for the growth of fecal coliforms in the cake samples but appears to provide the most toxic environment for samples in the presence of centrate.

- Post-dewatering, a higher proportion of extracellular ATP accumulates in the centrate as compared with the cake. The more powerful the centrifuge, the higher the concentrations of extracellular ATP can be. 
- For a longer sampling time, culturable fecal coliforms reach undetectable levels in cake and centrate samples whereas the total cell counts are still found to increase. These increases are therefore not due to fecal coliforms.

- The full-scale centrate from Treatment Plant A shows growth inhibition. After dewatering, samples in the absence of the centrate can show regrowth of their fecal coliforms.

- Fecal coliform counts from the treatment plant are not congruent with the changes in the total cell population, calculated with the ATP concentrations. Where culture counts stay steady, viable cell counts can change from one process to another.

\subsection{Future studies}

There are a few findings that deserve more attention and future studies on the subject the fate of fecal coliforms and the reactivation / regrowth of indicator organisms in centrifuge dewatered cake should touch upon the following subjects:

- A protocol should be established for the simulation of full-scale dewatering centrifuges in a laboratory setting.

- More comprehensive testing for the identification of the inhibitors that could be present in the centrate samples. This includes a more complete list of parameters for 
the chemical characterisation of centrate, such as the inclusion of the concentrations of heavy metals, as well as monitoring the effect of such concentrations individually on fecal coliform growth.

- Similar tests to those undertaken in this study should be undertaken with thermophilically digested sludge to see how the centrate post-dewatering compares with the centrate from a mesophilically digested plant and why the thermophilically digested sludge can have such large increases in fecal coliforms.

- More research should be done to determine if the Luminase method for viable cell count detection is ideal for cake samples and other modifications to the method should be tested. Other methods for viable cells counts could be undertaken, for these methods to be ideal, it should be able to quantify the viable counts of a particular type of cell or even identify a particular strain of the bacteria in order to make the comparison with the culture counts more worthwhile.

- The fate of other microorganisms through mechanical dewatering should be examined to see if they behave like fecal coliforms or more closely like the total cell population. Identifying what other indicator microorganisms, such as Salmonella, for which these increases could occur is important.

As more treatment plants observe post-digestion increases of indicator organisms, the topic of reactivation or regrowth of fecal coliforms is becoming increasingly important. This study has shown that regrowth is possible in mesophilically digested samples of 
centrifuged dewatered cake. The observations made here are added to a growing field where relatively little research has been done and the findings are forcing treatment plants to rethink their current sludge treatment and disposal strategy in order to remain in compliance and protect human health. 


\section{References}

Adams, B. L., Bates, T. C., \& Oliver, J. D. (2003). Survival of Helicobacter pylori in a natural freshwater environment. Applied and Environmental Microbiology, 69(12), 7462-7466

Allen, M. J., Edberg, S. C., \& Reasoner, D. J. (2004). Heterotrophic plate count bacteria what is their significance in drinking water? International Journal of Food Microbiology, 92(3), 265-274

American Public Health Association (APHA), American Water Works Association and Water Pollution Control Federation, (1989). Standard Methods for the Examination of Water and Wastewater. APHA / AWWA, Washington DC.

Arana, I., Seco, C., Epelde, K., Muela, A., Fernandez-Astorga, A., \& Barcina, I. (2004). Relationships between Escherichia coli cells and the surrounding medium during survival processes. Antonie Van Leeuwenhoek International Journal of General and Molecular Microbiology, 86(2), 189-199

Arbuckle, W. B., \& Alleman, J. E. (1992). Effluent toxicity testing using Nitrifiers and Microtox( $\left.{ }^{\mathrm{TM}}\right)$. Water Environment Research, 64(3), 263-267

Arretxe, M., Heap, J. M., \& Christofi, N. (1997). The effect of toxic discharges on ATP content in activated sludge. Environmental Toxicology and Water Quality, 12(1), 2329 
Artz, R. R. E., Avery, L. M., Jones, D. L., \& Killham, K. (2006). Potential pitfalls in the quantitative molecular detection of Escherichia coli $\mathrm{O} 157$ : H7 in environmental matrices. Canadian Journal of Microbiology, 52(5), 482-488

Aslan-Yilmaz, A., Okus, E., \& Ovez, S. (2004). Bacteriological indicators of anthropogenic impact prior to and during the recovery of water quality in an extremely polluted estuary, Golden Horn, Turkey. Marine Pollution Bulletin, 49(1112), 951-958

Azur Environmental, 1998. Microtox acute toxicity test.:1-23, Carlsbad, CA.

Baker, K. H., Redmond, B., \& Herson, D. S. (2005). False positive fecal coliform in biosolid samples assayed using A-1 medium. Water Environment Research, 77(4), 423-424

Barer, M. R., \& Harwood, C. R. (1999). Bacterial viability and culturability. Advances in Microbial Physiology, Vol 41, 41, 93-137

Baskerville, R. C., \& Gale, R. S. (1968). A simple automatic instrument for determining the filterability of sewage sludge. Water Pollution Control, 67, 233-241

Baumgartner, D. J., Glenn, E. P., Thompson, T. L., \& Skeen, B. A. (2005). Land disposal of centrate from biosolids production. Water Air and Soil Pollution, 162(1-4), 219228 
Bergeron, J., \& Paice, M. (2001). Monitoring of activated sludge systems using a combination of specific oxygen uptake rates and specific adenosine triphosphate measurements. Water Quality Research Journal of Canada, 36(4), 659-685

Bivins, J. L., \& Novak, J. T. (2001). Changes in dewatering properties between the thermophilic and mesophilic stages in temperature-phased anaerobic digestion systems. Water Environment Research, 73(4), 444-449

Bjergbaek, L. A., \& Roslev, P. (2005). Formation of nonculturable Escherichia coli in drinking water. Journal of Applied Microbiology, 99(5), 1090-1098

Bradford, M. M. (1976). Rapid and sensitive method for quantitation of microgram quantities of protein utilizing principle of protein-dye binding. Analytical Biochemistry, 72(1-2), 248-254

Brezonik, P. L., \& Patterson, .J.W. (1971). Activated sludge ATP - effects of environmental stress. Journal of the Sanitary Engineering Division-ASCE, 97(NSA6), 813-823

British Notes on Water Pollution, 1971. Inhibition in the anaerobic digestion process for sewage sludge. Water Research Centre, Stevenage, Hertz, Great Britain.

Cairns, J., 2007. LuminUltra ATP technology. Overcoming high-solids in cake samples for ATP technology use, personal communication 
Canadian Water and Wastewater Association. (2003). Water legislation and Substance/Quality database. , 2 2007, website: Http://www.cwwa.ca/legislation/faqs/biosolid.htm

Chang, L. L., Raudenbush, D. L., \& Dentel, S. K. (2001). Aerobic and anaerobic biodegradability of a flocculant polymer. Water Science and Technology, 44(2-3), $461-468$

Chen, Y. C., Higgins, M. J., Maas, N. A., \& Murthy, S. N. (2006). DNA extraction and Escherichia coli quantification of anaerobically digested biosolids using the competitive touchdown PCR method. Water Research, 40(16), 3037-3044

Dalzell, D. J. B., \& Christofi, N. (2002). An ATP luminescence method for direct toxicity assessment of pollutants impacting on the activated sewage sludge process. Water Research, 36(6), 1493-1502

Dan, T. B. B., Wynne, D., \& Manor, Y. (1997). Survival of enteric bacteria and viruses in Lake Kinneret, Israel. Water Research, 31(11), 2755-2760

Demirel, B., \& Yenigun, O. (2002). Two-phase anaerobic digestion processes: A review. Journal of Chemical Technology and Biotechnology, 77, 743-755

Dentel, S. K.,(2001). Conditioning. In: Sludge into Biosolids, L. Spinosa and P. A. Vesilind (Editors). IWA Publishing, London, UK, pp. 278-314 
Dentel, S. K., Abu-Orf, M. M., \& Walker, C. A. (2000). Optimization of slurry flocculation and dewatering based on electrokinetic and rheological phenomena. Chemical Engineering Journal, 80(1-3), 65-72

Diazburgos, M. A., Ceccanti, B., \& Polo, A. (1993). Monitoring biochemical-activity during sewage-sludge composting. Biology and Fertility of Soils, 16(2), 145-150

Eccles, J. P., Searle, R., Holt, D., \& Dennis, P. J. (2004). A comparison of methods used to enumerate Escherichia coli in conventionally treated sewage sludge. Journal of Applied Microbiology, 96(2), 375-383

El-Aassar, A., Kitcher, D., \& Forster, C. F. (1998). The release of heavy metals from digested sludge by centrifugation. Environmental Technology, 19(5), 537-542

Field, J. P., Farrell-Poe, K. L., \& Walworth, J. L. (2007). Comparative treatment effectiveness of conventional trench and seepage pit systems. Water Environment Research, 79(3), 310-319

Gauthier, M., 2000. Environmental Parameters Associated with the Viable but Nonculturable State. In: Nonculturable microorganisms in the environment, Rita R. Colwell and D. Jay Grimes (Editors). ASM Press, Washington, DC, pp. 87-112

Gilbert, P., Caplan, F., \& Brown, M. R. W. (1991). Centrifugation injury of gramnegative bacteria. Journal of Antimicrobial Chemotherapy, 27(4), 550-551

HACH Company, 2005a. Ammonia nitrogen: Salicylate method HR (0.4 to $50.0 \mathrm{mg} / \mathrm{L})$. Method 10031:1-4. Loveland, CO 
HACH Company, 2005b. Chemical Oxygen Demand: Reactor digester method UHR (200-15,000mg/L COD). Method 8000:1-8. Loveland, CO

HACH Company, 2005c. Nitrate: Chromotrophic acid method HR (0.2 to $30.0 \mathrm{mg} / \mathrm{L})$. Method 10020:1-4. Loveland, CO

HACH Company, 2005d. Nitrite: Diasotization method LR (0.05 to $2.00 \mathrm{mg} / \mathrm{L})$. Method 10207 TNT plus 839:1-4. Loveland, CO

HACH Company, 2005e. Reactive phosphorus (orthophosphate): Molybdovanadate method HR (1.0-100.0 mg/L). Method 8114:1-6. Loveland, CO

HACH Company, 2005f. Sulfide: Methylene blue method (5 to $800 \mathrm{ug} / \mathrm{L}$ ). Method 8131:1-4. Loveland, CO

HACH Company, 2005g. Total nitrogen: Persulfate digestion method HR (2 to 150 mg/L). Method 10072:1-8. Loveland, CO

HACH Company, 2005h. Total Organic Carbon: Direct method HR (100-700mg/L C). Method 10128:1-6. Loveland, CO

HACH Company, 2005i. Volatile acids: Esterification method (27-2800mg/L). Method 8196:1-6. Loveland, CO

Haveroen, M. E., MacKinnon, M. D., \& Fedorak, P. M. (2005). Polyacrylamide added as a nitrogen source stimulates methanogenesis in consortia from various wastewaters. Water Research, 39(14), 3333-3341 
Higgins, M., Y.C. Chen, \& Murthy, S. (2006). Understanding Factors Affecting Polymer Demand for Conditioning and Dewatering. Water Environment Federation and IWA Publishing, Alexandria, VA, United States of America.

Higgins, M. and S. Murthy, (2006). Examination of Reactivation and Regrowth of Fecal Coliforms in Centrifuge Dewatered, Anaerobically Digested Sludges. Water Environment Federation and IWA Publishing, Alexandria, VA, United States of America.

Higgins, M. J., Chen, Y. C., Murthy, S. N., Hendrickson, D., Farrel, J., \& Schafer, P. (2007). Reactivation and growth of non-culturable indicator bacteria in anaerobically digested biosolids after centrifuge dewatering. Water Research, 41(3), 665-673

Hwu, C. S., Donlon, B., \& Lettinga, G. (1996). Comparative toxicity of long-chain fatty acid to anaerobic sludges from various origins. Water Science and Technology, 34(56), 351-358

Iranpour, R., \& Cox, H. H. J. (2006). Recurrence of fecal coliforms and Salmonella species in biosolids following thermophilic anaerobic digestion. Water Environment Research, 78(9), 1005-1012

Iranpour, R., Cox, H. H. J., Oh, S., Fan, S., Kearney, R. J., Abkian, V. \& Haug, R.T. (2006). Thermophilic-anaerobic digestion to produce Class A biosolids: Initial fullscale studies at Hyperion treatment plant. Water Environment Research, 78(2), 170180 
Iranpour, R., Palacios, R., Cox, H. H. J., \& Abkian, V. (2005). Solving fecal coliform growth. Water Science and Technology, 52(1-2), 283-288

Jolis, D. (2006). Regrowth of fecal coliforms in Class A biosolids. Water Environment Research, 78(4), 442-445

Kell, D. B., Kaprelyants, A. S., Weichart, D. H., Harwood, C. R., \& Barer, M. R. (1998). Viability and activity in readily culturable bacteria: A review and discussion of the practical issues. Antonie Van Leeuwenhoek International Journal of General and Molecular Microbiology, 73(2), 169-187

Khanna, M. R., Bhavsar, S. P., \& Kapadnis, B. P. (2006). Effect of temperature on growth and chemotactic behaviour of Campylobacter jejuni. Letters in Applied Microbiology, 43(1), 84-90

Koehler, L. H. (1952). Differentiation of carbohydrates by Anthrone reaction rate and color intensity. Analytical Chemistry, 24(10), 1576-1579

Lalman, J. A., \& Bagley, D. M. (2001). Anaerobic degradation and methanogenic inhibitory effects of oleic and stearic acids. Water research, 35(12), 2975-2983

Lewis, A., (2006). Regulatory framework for biosolids management in Canada. Greater Vancouver Regional District:1-19.

Lleo, M. M., Bonato, B., Tafi, M. C., Signoretto, C., Boaretti, M., \& Canepari, P. (2001). Resuscitation rate in different enterococcal species in the viable but non-culturable state. Journal of Applied Microbiology, 91(6), 1095-1102 
Londry, K. L., \& Suflita, J. M. (1999). Use of nitrate to control sulfide generation by sulfate-reducing bacteria associated with oily waste. Journal of Industrial Microbiology \& Biotechnology, 22(6), 582-589

LuminUltra Technologies, 2004. ATP - A reliable indicator of active biomass.:1-2. Fredericton, NB

LuminUltra Technologies, 2007. TCB quick-reference guide. Version 3.0:1-2. Fredericton, NB

Mallin, M. A., Williams, K. E., Esham, E. C., \& Lowe, R. P. (2000). Effect of human development on bacteriological water quality in coastal watersheds. Ecological Applications, 10(4), 1047-1056

Marin, J. A., Hernandez, T., \& Garcia, C. (2005). Bioremediation of oil refinery sludge by landfarming in semiarid conditions: Influence on soil microbial activity. Environmental Research, 98(2), 185-195

McDougald, D., Rice, S. A., Weichart, D., \& Kjelleberg, S. (1998). Nonculturability: Adaptation or debilitation? FEMS Microbiology Ecology, 25(1), 1-9

Mckay, A. M. (1992). Viable but non-culturable forms of potentially pathogenic bacteria in water. Letters in Applied Microbiology, 14(4), 129-135

Metcalf \& Eddy Inc., 2003. Wastewater engineering : treatment and reuse. McGraw Hill, Boston, MA. 
MicroBiologics, 2004. Kwik-Stik methodology: Simply efficient. LIT 095:1-1. Ottawa, $\mathrm{ON}$

Mizunoe, Y., Wai, S. N., Takade, A., \& Yoshida, S. (1999). Restoration of culturability of starvation-stressed and low-temperature-stressed Escherichia coli $\mathrm{O} 157$ cells by using $\mathrm{H}_{2} \mathrm{O}_{2}$-degrading compounds. Archives of Microbiology, 172(1), 63-67

Monteleone, M. C., Furness, D., Jefferson, B., \& Cartmell, E. (2004). Fate of $E$ coli across mechanical dewatering processes. Environmental Technology, 25(7), 825-831

Morris, D. L. (1948). Quantitative determination of carbohydrates with Dreywoods Anthrone reagent. Science, 107(2775), 254-255

Morrison, G. A., \& Hinshelwood, C. (1949). Nitrogen utilisation and growth of coliform bacteria .3. nitrogen utilisation and lag phase. Journal of the Chemical Society 1949 (FEB), 380-384

Mossakowska, A., Reinius, L. G., \& Hultman, B. (1997). Nitrification reactions in treatment of supernatant from dewatering of digested sludge. Water Environment Research, 69(6), 1128-1133

Na, S. H., Miyanaga, K., Unno, H., \& Tanji, Y. (2006). The survival response of Escherichia coli $\mathrm{K} 12$ in a natural environment. Applied Microbiology and Biotechnology, 72(2), 386-392 
Nelson, S. M., Attwell, R. W., Dawson, M. M., \& Smith, C. A. (1996). The effect of temperature on viability of carbon- and nitrogen-starved Escherichia coli. Microbial Ecology, 32(1), 11-21

Nicolau, A., Mota, M., \& Lima, N. (2004). Effect of different toxic compounds on ATP content and acid phosphatase activity in axenic cultures of Tetrahymena pyriformis. Ecotoxicology and Environmental Safety, 57(2), 129-135

Nova Scotia: Environment and Labour. (2004). Guidelines for land application and storage of biosolids in Nova Scotia. , Halifax, NS, 1-22

Novak, J. T., 2001. Dewatering. In: Sludge into Biosolids, L. Spinosa and P. A. Vesilind (Editors). IWA Publishing, London, UK, pp. 339-363

Novak, J. T. (2006). Dewatering of sewage sludge. Drying Technology, 24(10), 1257 1262

Novak, J. T., \& Lynch, D. P. (1990). The effect of shear on conditioning - chemical requirements during mechanical sludge dewatering. Water Science and Technology, $22(12), 117-124$

Ohge, H., Furne, J. K., Springfield, J., Rothenberger, D. A., Madoff, R. D., \& Levitt, M. D. (2005). Association between fecal hydrogen sulfide production and pouchitis. Diseases of the Colon \& Rectum, 48(3), 469-475 
Oleszkiewicz, J. A., \& Mavinic, D. S. (2001). Wastewater biosolids: An overview of processing, treatment, and management. Canadian Journal of Civil Engineering, 28, $102-114$

Oliver, J. D., 1993. Formation of viable but nonculturable cells. In: Starvation in bacteria,Staffan Kjelleberg (editor). Plenum Press, New York, pp. 239-272

Oliver, J. D., Hite, F., Mcdougald, D., Andon, N. L., \& Simpson, L. M. (1995). Entry into, and resuscitation from, the viable but nonculturable state by Vibrio-vulnificus in an estuarine environment. Applied and Environmental Microbiology, 61(7), 26242630

Örmeci, B. (2007). Optimization of a full-scale dewatering operation based on the rheological characteristics of wastewater sludge. Water Research, 41(6), 1243-1252

Örmeci, B., \& Linden, K. G. (2005). Comparison of physical and chemical methods for extraction of coliform from wastewater particles and flocs. Environmental Engineering Science, 22(4), 459-471

Pacheco, C. C., Alves, C. C., Barreiros, L., Castro, P. M. L., \& Teixeira, P. C. M. (2003). Epifluorescence microscope methods for bacterial enumeration in a 4-chlorophenol degrading consortium. Biotechnology Letters, 25(24), 2089-2092

Parkin, G. F., \& Owen, W. F. (1986). Fundamentals of anaerobic digestion of wastewater sludges. Journal of Environmental Engineering, 112(5), 867-920 
Pembrey, R. S., Marshall, K. C., \& Schneider, R. P. (1999). Cell surface analysis techniques: What do cell preparation protocols do to cell surface properties? Applied and Environmental Microbiology, 65(7), 2877-2894

Pierce, 2004. Instructions: Coomassie (Bradford) protein assay kit. 23200:1-7. Rockford, IL

Prieme, A., Sitaula, J. I. B., Klemedtsson, A. K., \& Bakken, L. R. (1996). Extraction of methane-oxidizing bacteria from soil particles. FEMS Microbiology Ecology, 21(1), $59-68$

Qi, Y. N., Dentel, S. K., \& Herson, D. S. (2007). Increases in fecal coliform bacteria resulting from centrifugal dewatering of digested biosolids. Water Research, 41(3), $571-580$

Qi, Y. N., Gillow, S., Herson, D. S., \& Dentel, S. K. (2004). Reactivation and/or growth of fecal coliform bacteria during centrifugal dewatering of anaerobically digested biosolids. Water Science and Technology, 50(9), 115-120

Ravel, J., Knight, I. T., Monahan, C. E., Hill, R. T., \& Colwell, R. R. (1995).

Temperature-induced recovery of Vibrio-cholerae from the viable but nonculturable state - growth or resuscitation. Microbiology-UK, 141, 377-383

Reissbrodt, R., Rienaecker, I., Romanova, J. A., Freestone, P. P. E., Haigh, R. D., Lyte, A., Tschape, H. \& Williams, P.H. (2002). Resuscitation of Salmonella enterica serovar Typhimurium and enterohemorrhagic Escherichia coli from the viable but 
nonculturable state by heat-stable enterobacterial autoinducer. Applied and Environmental Microbiology, 68(10), 4788-4794

Ren, S. J., \& Frymier, P. D. (2004). Statistical comparison of bioassays for assessment of toxicity of organic components of wastewater to activated sludge. Journal of Environmental Engineering-ASCE, 130(4), 484-488

Ricco, G., Tomei, M. C., Ramadori, R., \& Laera, G. (2004). Toxicity assessment of common xenobiotic compounds on municipal activated sludge: Comparison between respirometry and Microtox (R). Water Research, 38(8), 2103-2110

Rigsbee, W., Simpson, L. M., \& Oliver, J. D. (1997). Detection of the viable but nonculturable state in Escherichia coli O157:H7. Journal of Food Safety, 16(4), 255262

Rinzema, A., Boone, M., Vanknippenberg, K., \& Lettinga, G. (1994). Bactericidal effect of long-chain fatty-acids in anaerobic-digestion. Water Environment Research, $66(1), 40-49$

Rosso, L., Lobry, J. R., Bajard, S., \& Flandrois, J. P. (1995). Convenient model to describe the combined effects of temperature and $\mathrm{pH}$ on microbial-growth. Applied and Environmental Microbiology, 61(2), 610-616

Roth, W. G., Leckie, M. P., \& Dietzler, D. N. (1988). Restoration of colony-forming activity in osmotically stressed Escherichia-coli by Betaine. Applied and Environmental Microbiology, 54(12), 3142-3146 
Rudolfs, W., \& Amberg, H. R. (1952). White water treatment .2. Effect of sulfides on digestion. Sewage and Industrial Wastes, 24(10), 1278-1287

Santha, H., Sandino, J., Shimp, G. F., \& Sung, S. W. (2006). Performance evaluation of a 'sequential-batch' temperature-phased anaerobic digestion (TPAD) scheme for producing Class A biosolids. Water Environment Research, 78(3), 221-226

Spellman, F. R., 1997. Dewatering Biosolids. Technomic Publishing Company Inc, Lancaster, PA, USA.

Stotzky, G., 1985. Mechanisms of adhesion to clays, with reference to soil systems. In: Bacterial adhesion: Mechanisms and physiological significance, D. C. Savage and M. Fletcher (Editors). Plenum Press, New York, pp. 195-254

Task Force on Anaerobic Sludge Digestion, 1987. Anaerobic Sludge Digestion. Water Pollution Control Federation, Alexandria, VA.

Task Force on Sludge Conditioning, 1988. Sludge Conditioning. Water Pollution Control Federation, Alexandria VA.

Task Force on Sludge Dewatering, 1983. Sludge Dewatering. Water Pollution Control Federation, Washington D.C.

Task Force on Sludge Thickening, 1980. Sludge Thickening. Water Polllution Control Federation, Alexandria, VA.

Task Force on Wastewater Residuals Stabilization, 1995. Wastewater Residuals Stabilization. Water Environment Federation, Alexandria VA. 
Thorndahl, U. (1993). Nitrogen removal from returned liquors. Journal of the Institution of Water and Environmental Management, 7(5), 492-496

Tree, J. A., Adams, M. R., \& Lees, D. N. (2003). Chlorination of indicator bacteria and viruses in primary sewage effluent. Applied and Environmental Microbiology, 69(4), 2038-2043

United States Environmental Protection Agency (US EPA), 1982. Dewatering Municipal Wastewater Sludges: Design Manual. United States Environmental Protection Agency, Washington, D.C.

United States Environmental Protection Agency (US EPA), 1994. A Plain English Guide to the EPA Part 503 Biosolids Rule. United States Environmental Protection Agency, Washington, D.C.

United States Environmental Protection Agency (US EPA), 2005a. Method 1680: Fecal Coliforms in Sewage Sludge (Biosolids) by Multiple Tube Fermentation using Lauryl Tryptose Broth (LTB) and EC Medium. US EPA, Washington DC.

United States Environmental Protection Agency (US EPA), 2005b. Method 1681: Fecal Coliforms in Sewage Sludge (Biosolids) by Multiple Tube Fermentation using A-1 Medium . US EPA, Washington DC.

Vesilind, P. A., G.C. Hartman, and E.T. Skene, 1986. Sludge Management and Disposal for the Practicing Engineer. Lewis Publishers, Inc, Chelsea, MI, pp. 314 
Vesilind, P. A. \& Spinosa, L., 2001. Production and regulations. In: Sludge into Biosolids, L. Spinosa and P. A. Vesilind (Editors). IWA Publishing, London, UK, pp. 3-18

Weemaes, M. \& Verstraete, W., 2001. Other Treatment Techniques. In: Sludge into Biosolids, L. Spinosa and P. A. Vesilind (Editors). IWA Publishing, London, UK, pp. 364-383

Wery, N., Pourcher, A. M., Stan, V., Delgenes, J. P., Picard-Bonnaud, F., \& Godon, J. J. (2006). Survival of Listeria monocytogenes and Enterococcus faecium in sludge evaluated by real-time PCR and culture methods. Letters in Applied Microbiology, $43(2), 131-136$ 


\section{Appendix A: Raw Data}

\section{Appendix A1: Treatment plant data}

Figure 1.3: $E$ coli concentrations from Treatment plant A

\begin{tabular}{|c|c|c|c|c|c|c|c|}
\hline \multicolumn{8}{|c|}{2004} \\
\hline \multicolumn{4}{|c|}{ Digester } & \multicolumn{4}{|c|}{ Cake } \\
\hline $\mathrm{cfu} / 100 \mathrm{~mL}$ & $\begin{array}{c}\log \text { (cfu } / \mathbf{g} \\
\text { wet) }\end{array}$ & cfu/g DW & $\begin{array}{c}\log (\mathrm{cfu} / \mathrm{g} \\
\text { DW) }\end{array}$ & cfu/g wet & $\begin{array}{c}\log \text { (cfu } / \\
\text { g wet) }\end{array}$ & cfu/gDW & $\begin{array}{c}\log (\mathrm{cfu} / \mathrm{g} \\
\text { DW) }\end{array}$ \\
\hline 55000 & 2.740 & 18333 & 4.263 & 17900 & 4.253 & 59667 & $\bar{~} 4.776$ \\
\hline 44000 & 2.643 & 14667 & 4.166 & 50000 & 4.699 & 166667 & 5.222 \\
\hline 48000 & 2.681 & 16000 & 4.204 & 89700 & 4.953 & 299000 & 5.476 \\
\hline 50000 & 2.699 & 16667 & 4.222 & 27600 & 4.441 & 92000 & 4.964 \\
\hline 56000 & 2.748 & 18667 & 4.271 & 3205757 & 6.506 & 10685857 & 7.029 \\
\hline 31000 & 2.491 & 10333 & 4.014 & 13035 & 4.115 & 43450 & 4.638 \\
\hline 47000 & 2.672 & 15667 & 4. 195 & 1000000 & 6.000 & 3333333 & 6.523 \\
\hline 51000 & 2.708 & 17000 & 4.230 & 33000 & 4.519 & 110000 & 5.041 \\
\hline 50000 & 2.699 & 16667 & 4.222 & 1516 & 3.181 & 5053 & 3.704 \\
\hline 73000 & 2.863 & 24333 & 4.386 & 9482 & 3.977 & 31607 & 4.500 \\
\hline 37000 & 2.568 & 12333 & 4.091 & 93103 & 4.969 & 310343 & 5.492 \\
\hline 57000 & 2.756 & 19000 & 4.279 & 73333 & 4.865 & 244443 & 5.388 \\
\hline 63000 & 2.799 & 21000 & 4.322 & 90740 & 4.958 & 302467 & 5.481 \\
\hline 100000 & 3.000 & 33333 & 4.523 & 85000 & 4.929 & 283333 & 5.452 \\
\hline 57000 & 2.756 & 19000 & 4.279 & 1568 & 3.195 & 5227 & 3.718 \\
\hline 58000 & 2.763 & 19333 & 4.286 & 1516 & 3.181 & 5053 & 3.704 \\
\hline 38000 & 2.580 & 12667 & 4.103 & 1612 & 3.207 & 5373 & 3.730 \\
\hline 42000 & 2.623 & 14000 & 4.146 & 1562 & 3.194 & 5207 & 3.717 \\
\hline 55000 & 2.740 & 18333 & 4.263 & 11406 & 4.057 & 38020 & 4.580 \\
\hline 28000 & 2.447 & 9333 & 3.970 & 1562 & 3.194 & 5207 & 3.717 \\
\hline 51000 & 2.708 & 17000 & 4.230 & 2840 & 3.453 & 9467 & 3.976 \\
\hline 61000 & 2.785 & 20333 & 4.308 & 1515 & 3.180 & 5050 & 3.703 \\
\hline 75000 & 2.875 & 25000 & 4.398 & 1515 & 3.180 & 5050 & 3.703 \\
\hline 55000 & 2.740 & 18333 & 4.263 & 1612 & 3.207 & 5373 & 3.730 \\
\hline 8600000 & 4.934 & 2866667 & 6.457 & 1666 & 3.222 & 5553 & 3.745 \\
\hline 85000 & 2.929 & 28333 & 4.452 & 2935 & 3.468 & 9783 & 3.990 \\
\hline 85000 & 2.929 & 28333 & 4.452 & 21935 & 4.341 & 73117 & 4.864 \\
\hline 33000 & 2.519 & 11000 & 4.041 & 1574 & 3.197 & 5247 & 3.720 \\
\hline 410000 & 3.613 & 136667 & 5.136 & 58333 & 4.766 & 194443 & 5.289 \\
\hline 85000 & 2.929 & 28333 & 4.452 & 25000 & 4.398 & 83333 & 4.921 \\
\hline 54000 & 2.732 & 18000 & 4.255 & 400000 & 5.602 & 1333333 & 6.125 \\
\hline 78000 & 2.892 & 26000 & 4.415 & 783333 & 5.894 & 2611110 & 6.417 \\
\hline 90000 & 2.954 & 30000 & 4.477 & 6000 & 3.778 & 20000 & 4.301 \\
\hline 64000 & 2.806 & 21333 & 4.329 & 67241 & 4.828 & 224137 & 5.351 \\
\hline 140000 & 3.146 & 46667 & 4.669 & 83333 & 4.921 & 277777 & 5.444 \\
\hline 230000 & 3.362 & 76667 & 4.885 & 90000 & 4.954 & 300000 & 5.477 \\
\hline 51000 & 2.708 & 17000 & 4.230 & 283333 & 5.452 & 944443 & 5.975 \\
\hline 65000 & 2.813 & 21667 & 4.336 & 171428 & 5.234 & 571427 & 5.757 \\
\hline 20000 & 2.301 & 6667 & 3.824 & 741379 & 5.870 & 2471263 & 6.393 \\
\hline 81000 & 2.908 & 27000 & 4.431 & 1133333 & 6.054 & 3777777 & 6.577 \\
\hline 82000 & 2.914 & 27333 & 4.437 & 73214 & 4.865 & 244047 & 5.387 \\
\hline 32000 & 2.505 & 10667 & 4.028 & 160714 & 5.206 & 535713 & 5.729 \\
\hline 62000 & 2.792 & 20667 & 4.315 & 181481 & 5.259 & 604937 & 5.782 \\
\hline 42000 & 2.623 & 14000 & 4.146 & & & & \\
\hline 47000 & 2.672 & 15667 & 4.195 & & & & \\
\hline
\end{tabular}


Figure 1.3: $E$ coli concentrations from Treatment plant $A$

\begin{tabular}{|c|c|c|c|c|c|c|c|}
\hline \multicolumn{8}{|c|}{2005} \\
\hline \multicolumn{4}{|c|}{ Digester } & \multicolumn{4}{|c|}{ Cake } \\
\hline cfu/100mL & $\begin{array}{c}\log \text { (cfu } I \\
\text { g wet) }\end{array}$ & cfu/g DW & $\begin{array}{c}\log (\mathrm{cfu} / \mathrm{g} \\
\text { DW) }\end{array}$ & cfu/g wet & $\begin{array}{c}\log \text { (cfu } / \\
\text { g wet) }\end{array}$ & cfu/gDW & $\begin{array}{c}\log \text { (cfu } 1 \\
\text { g DW) }\end{array}$ \\
\hline 42000 & 2.623 & 14000 & 4.146 & 7500000 & 6.875 & 25000000 & 7.398 \\
\hline 33000 & 2.519 & 11000 & 4.041 & 3400000 & 6.531 & 11333333 & 7.054 \\
\hline 14500 & 2.161 & 4833 & 3.684 & 26900 & 4.430 & 89667 & 4.953 \\
\hline 28000 & 2.447 & 9333 & 3.970 & 37000 & 4.568 & 123333 & 5.091 \\
\hline 30000 & 2.477 & 10000 & 4.000 & 50000 & 4.699 & 166667 & 5.222 \\
\hline 41000 & 2.613 & 13667 & 4.136 & 29300 & 4.467 & 97667 & 4.990 \\
\hline 53000 & 2.724 & 17667 & 4.247 & 38300 & 4.583 & 127667 & 5.106 \\
\hline 61000 & 2.785 & 20333 & 4.308 & 126000 & 5.100 & 420000 & 5.623 \\
\hline 54000 & 2.732 & 18000 & 4.255 & 150000 & 5.176 & 500000 & 5.699 \\
\hline 60000 & 2.778 & 20000 & 4.301 & 38000 & 4.580 & 126667 & 5.103 \\
\hline 46000 & 2.663 & 15333 & 4.186 & 53300 & 4.727 & 177667 & 5.250 \\
\hline 48000 & 2.681 & 16000 & 4.204 & 147000 & 5.167 & 490000 & 5.690 \\
\hline 65000 & 2.813 & 21667 & 4.336 & 36700 & 4.565 & 122333 & 5.088 \\
\hline 76000 & 2.881 & 25333 & 4.404 & 37500 & 4.574 & 125000 & 5.097 \\
\hline 38000 & 2.580 & 12667 & 4.103 & 30000 & 4.477 & 100000 & 5.000 \\
\hline 21000 & 2.322 & 7000 & 3.845 & 610000 & 5.785 & 2033333 & 6.308 \\
\hline 63000 & 2.799 & 21000 & 4.322 & 448000 & 5.651 & 1493333 & 6.174 \\
\hline 54000 & 2.732 & 18000 & 4.255 & 152000 & 5.182 & 506667 & 5.705 \\
\hline 35000 & 2.544 & 11667 & 4.067 & 328000 & 5.516 & 1093333 & 6.039 \\
\hline 41000 & 2.613 & 13667 & 4.136 & 236000 & 5.373 & 786667 & 5.896 \\
\hline 53000 & 2.724 & 17667 & 4.247 & 5500 & 3.740 & 18333 & 4.263 \\
\hline 51000 & 2.708 & 17000 & 4.230 & 5500 & 3.740 & 18333 & 4.263 \\
\hline 45000 & 2.653 & 15000 & 4.176 & 4500 & 3.653 & 15000 & 4.176 \\
\hline 30000 & 2.477 & 10000 & 4.000 & 21000 & 4.322 & 70000 & 4.845 \\
\hline 32000 & 2.505 & 10667 & 4.028 & 2700 & 3.431 & 9000 & 3.954 \\
\hline 47000 & 2.672 & 15667 & 4.195 & 5500 & 3.740 & 18333 & 4.263 \\
\hline 68000 & 2.833 & 22667 & 4.355 & 1820 & 3.260 & 6067 & 3.783 \\
\hline 140000 & 3.146 & 46667 & 4.669 & 6400 & 3.806 & 21333 & 4.329 \\
\hline 98000 & 2.991 & 32667 & 4.514 & 12700 & 4.104 & 42333 & 4.627 \\
\hline 65000 & 2.813 & 21667 & 4.336 & 1000 & 3.000 & 3333 & 3.523 \\
\hline 47000 & 2.672 & 15667 & 4.195 & 23000 & 4.362 & 76667 & 4.885 \\
\hline 180000 & 3.255 & 60000 & 4.778 & 13600 & 4.134 & 45333 & 4.656 \\
\hline 97000 & 2.987 & 32333 & 4.510 & 14500 & 4.161 & 48333 & 4.684 \\
\hline 87000 & 2.940 & 29000 & 4.462 & 13600 & 4.134 & 45333 & 4.656 \\
\hline 63000 & 2.799 & 21000 & 4.322 & 46000 & 4.663 & 153333 & 5.186 \\
\hline 96000 & 2.982 & 32000 & 4.505 & 15500 & 4.190 & 51667 & 4.713 \\
\hline 160000 & 3.204 & 53333 & 4.727 & 16400 & 4.215 & 54667 & 4.738 \\
\hline 75000 & 2.875 & 25000 & 4.398 & & & & \\
\hline 51000 & 2.708 & 17000 & 4.230 & & & & \\
\hline 6300 & 1.799 & 2100 & 3.322 & & & & \\
\hline
\end{tabular}


Figure 1.3: $E$ coli concentrations from Treatment plant A

\begin{tabular}{|c|c|c|c|c|c|c|c|}
\hline \multicolumn{8}{|c|}{2006} \\
\hline \multicolumn{4}{|c|}{ Digester } & \multicolumn{4}{|c|}{ Cake } \\
\hline cfu/100mL & $\begin{array}{c}\log \text { (cfu } / \\
\text { g wet) }\end{array}$ & cfu/g DW & $\begin{array}{c}\log (\mathrm{cfu} / \\
\text { g DW) }\end{array}$ & cfu/g wet & $\begin{array}{c}\log \text { (cfu } / \\
\text { g wet) }\end{array}$ & cfu/gDW & $\begin{array}{c}\log \text { (cfu } / \\
\text { g DW) }\end{array}$ \\
\hline 62000 & 2.792 & 20667 & 4.315 & 46400 & 4.667 & 154667 & 5.189 \\
\hline 58000 & 2.763 & 19333 & 4.286 & 71400 & 4.854 & 238000 & 5.377 \\
\hline 30000 & 2.477 & 10000 & 4.000 & 928000 & 5.968 & 3093333 & 6.490 \\
\hline 62000 & 2.792 & 20667 & 4.315 & 121000 & 5.083 & 403333 & 5.606 \\
\hline 47000 & 2.672 & 15667 & 4.195 & 6920 & 3.840 & 23067 & 4.363 \\
\hline 21000 & 2.322 & 7000 & 3.845 & 68600 & 4.836 & 228667 & 5.359 \\
\hline 49000 & 2.690 & 16333 & 4.213 & 89300 & 4.951 & 297667 & 5.474 \\
\hline 52000 & 2.716 & 17333 & 4.239 & 55000 & 4.740 & 183333 & 5.263 \\
\hline 56000 & 2.748 & 18667 & 4.271 & 34900 & 4.543 & 116333 & 5.066 \\
\hline 45000 & 2.653 & 15000 & 4.176 & 91800 & 4.963 & 306000 & 5.486 \\
\hline 59000 & 2.771 & 19667 & 4.294 & 110700 & 5.044 & 369000 & 5.567 \\
\hline 44000 & 2.643 & 14667 & 4.166 & 8200 & 3.914 & 27333 & 4.437 \\
\hline 51000 & 2.708 & 17000 & 4.230 & 59400 & 4.774 & 198000 & 5.297 \\
\hline 46000 & 2.663 & 15333 & 4.186 & 39200 & 4.593 & 130667 & 5.116 \\
\hline 55000 & 2.740 & 18333 & 4.263 & 54272 & 4.735 & 180907 & 5.257 \\
\hline 38000 & 2.580 & 12667 & 4.103 & 7430000 & 6.871 & 24766667 & 7.394 \\
\hline 54000 & 2.732 & 18000 & 4.255 & 51567 & 4.712 & 171890 & 5.235 \\
\hline 40000 & 2.602 & 13333 & 4.125 & 17300 & 4.238 & 57667 & 4.761 \\
\hline 73000 & 2.863 & 24333 & 4.386 & 92900 & 4.968 & 309667 & 5.491 \\
\hline 87000 & 2.940 & 29000 & 4.462 & 33300 & 4.522 & 111000 & 5.045 \\
\hline 55000 & 2.740 & 18333 & 4.263 & 40800 & 4.611 & 136000 & 5.134 \\
\hline 40000 & 2.602 & 13333 & 4.125 & 99800 & 4.999 & 332667 & 5.522 \\
\hline 45000 & 2.653 & 15000 & 4.176 & 53500 & 4.728 & 178333 & 5.251 \\
\hline 16400 & 2.215 & 5467 & 3.738 & 13990 & 4.146 & 46633 & 4.669 \\
\hline 43000 & 2.633 & 14333 & 4.156 & 69600 & 4.843 & 232000 & 5.365 \\
\hline 54000 & 2.732 & 18000 & 4.255 & 49500 & 4.695 & 165000 & 5.217 \\
\hline 61000 & 2.785 & 20333 & 4.308 & 36690 & 4.565 & 122300 & 5.087 \\
\hline 52000 & 2.716 & 17333 & 4.239 & 110000 & 5.041 & 366667 & 5.564 \\
\hline 88000 & 2.944 & 29333 & 4.467 & 100000 & 5.000 & 333333 & 5.523 \\
\hline
\end{tabular}


Figure 5.1: Centrifuge speed and LTB / EC Media

\begin{tabular}{|c|c|c|c|c|c|c|c|c|c|}
\hline $\begin{array}{c}\text { Sample } \\
\text { type }\end{array}$ & $\begin{array}{c}\text { Polymer } \\
\text { dose }\end{array}$ & Day & $\begin{array}{c}\text { Centrifuge } \\
\text { speed } \\
\text { (rpm) }\end{array}$ & $\begin{array}{l}\text { Total } \\
\text { solids }\end{array}$ & $\begin{array}{c}\text { MPN/g } \\
\text { wet } \\
\text { weight }\end{array}$ & $\begin{array}{c}\mathbf{M P N} / \mathrm{g} \\
\mathrm{DW}\end{array}$ & $\begin{array}{c}\text { log (MPN } \\
\text { / g wet } \\
\text { weight) }\end{array}$ & $\begin{array}{c}\log \\
(\mathrm{MPN} / \mathrm{g} \\
\text { DW) }\end{array}$ & $\begin{array}{c}95 \% \log \\
\text { MPN }\end{array}$ \\
\hline Digested & 0 & 0 & 0 & 0.025 & 3291 & 132579 & 3.517 & 5.122 & 0.446 \\
\hline Centrate & 0 & 0 & 4500 & 0.004 & 3477 & 880471 & 3.541 & 5.945 & 0.463 \\
\hline Cake & 0 & 0 & 4500 & 0.105 & 2312 & 22119 & 3.364 & 4.345 & 0.421 \\
\hline Centrate & 0 & 0 & 3500 & 0.004 & 128 & 29841 & 2.106 & 4.475 & 0.436 \\
\hline Cake & 0 & 0 & 3500 & 0.069 & 2161 & 31385 & 3.335 & 4.497 & 0.420 \\
\hline Centrate & 0 & 0 & 2500 & 0.004 & 169 & 38188 & 2.228 & 4.582 & 0.421 \\
\hline Cake & 0 & 0 & 2500 & 0.050 & 1689 & 33933 & 3.228 & 4.531 & 0.421 \\
\hline Digested & 0 & 1 & 0 & 0.024 & 447 & 18306 & 2.650 & 4.263 & 0.600 \\
\hline Centrate & 0 & 1 & 4500 & 0.004 & 9178 & 2261409 & 3.963 & 6.354 & 0.505 \\
\hline Cake & 0 & 1 & 4500 & 0.102 & 160900 & 1575638 & 5.207 & 6.197 & 0.473 \\
\hline Centrate & 0 & 1 & 3500 & 0.005 & 78 & 17060 & 1.891 & 4.232 & 0.493 \\
\hline Cake & 0 & 1 & 3500 & 0.082 & 1689 & 20478 & 3.228 & 4.311 & 0.421 \\
\hline Centrate & 0 & 1 & 2500 & 0.005 & 78 & 16483 & 1.891 & 4.217 & 0.493 \\
\hline Cake & 0 & 1 & 2500 & 0.060 & 778 & 13032 & 2.891 & 4.115 & 0.493 \\
\hline
\end{tabular}




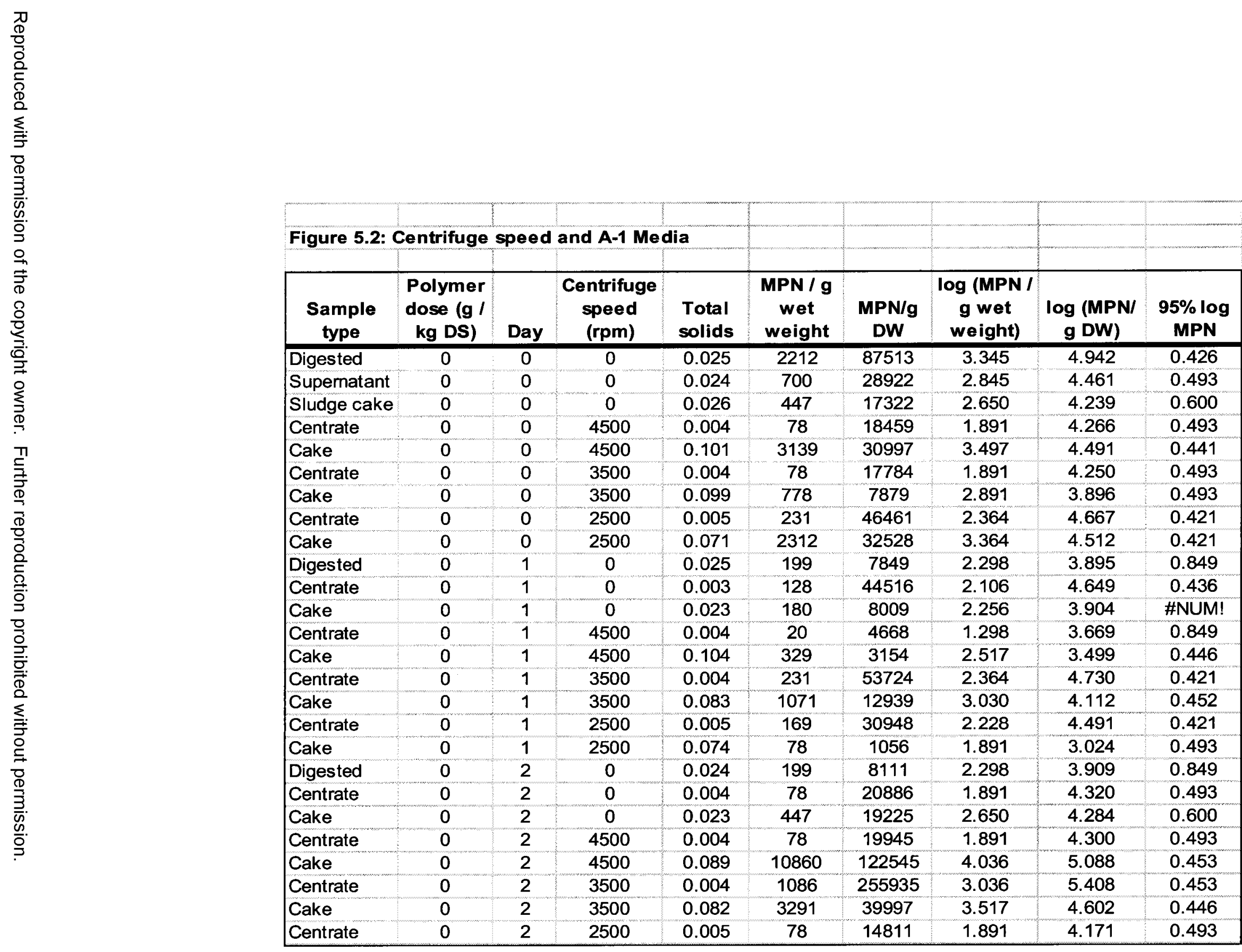


Figure 5.3: Centrifuge Speed, A-1 Media, CA 4600 polymer

\begin{tabular}{|c|c|c|c|c|c|c|c|c|c|}
\hline $\begin{array}{c}\text { Sample } \\
\text { type }\end{array}$ & $\begin{array}{c}\text { Polymer } \\
\text { dose (g / } \\
\text { kg DS) }\end{array}$ & Day & $\begin{array}{c}\text { Centrifuge } \\
\text { speed } \\
\text { (rpm) }\end{array}$ & $\begin{array}{l}\text { Total } \\
\text { solids }\end{array}$ & $\begin{array}{c}\text { MPN/g } \\
\text { wet } \\
\text { weight }\end{array}$ & $\begin{array}{c}\mathbf{M P N} / \mathbf{g} \\
\text { DW }\end{array}$ & $\begin{array}{c}\text { log (MPN / } \\
\text { g wet } \\
\text { weight) }\end{array}$ & $\begin{array}{c}\log (M P N / \\
g(D W)\end{array}$ & $\begin{array}{c}95 \% \log \\
\text { MPN }\end{array}$ \\
\hline Digested & 15 & 0 & 0 & 0.025 & 12990 & 526656 & 4.114 & 5.722 & 0.438 \\
\hline Supernatant & 15 & 0 & 0 & 0.002 & 493 & 197733 & 2.693 & 5.296 & 0.489 \\
\hline Sludge cake & 15 & 0 & 0 & 0.033 & 4932 & 150240 & 3.693 & 5.177 & 0.489 \\
\hline Centrate & 15 & 0 & 4500 & 0.002 & 70 & 40468 & 1.845 & 4.607 & 0.493 \\
\hline Cake & 15 & 0 & 4500 & 0.131 & 3291 & 25148 & 3.517 & 4.401 & 0.446 \\
\hline Centrate & 15 & 0 & 3500 & 0.002 & 240 & 113108 & 2.380 & 5.053 & 0.429 \\
\hline Cake & 15 & 0 & 3500 & 0.116 & 3291 & 28323 & 3.517 & 4.452 & 0.446 \\
\hline Centrate & 15 & 0 & 2500 & 0.002 & 221 & 101280 & 2.345 & 5.006 & 0.426 \\
\hline Cake & 15 & 0 & 2500 & 0.103 & 4932 & 47879 & 3.693 & 4.680 & 0.489 \\
\hline Centrate & 15 & 1 & 0 & 0.004 & 792 & 194518 & 2.899 & 5.289 & 0.484 \\
\hline Cake & 15 & 1 & 0 & 0.040 & 12990 & 325205 & 4.114 & 5.512 & 0.438 \\
\hline Centrate & 15 & 1 & 4500 & 0.002 & 70 & 35479 & 1.845 & 4.550 & 0.493 \\
\hline Cake & 15 & 1 & 4500 & 0.128 & 4469 & 34952 & 3.650 & 4.543 & 0.600 \\
\hline Centrate & 15 & 1 & 3500 & 0.002 & 23 & 10951 & 1.364 & 4.039 & 0.421 \\
\hline Cake & 15 & 1 & 3500 & 0.119 & 1987 & 16743 & 3.298 & 4.224 & 0.849 \\
\hline Centrate & 15 & 1 & 2500 & 0.002 & 542 & 316031 & 2.734 & 5.500 & 0.519 \\
\hline Cake & 15 & 1 & 2500 & 0.107 & 13840 & 129303 & 4.141 & 5.112 & 0.430 \\
\hline Centrate & 15 & 2 & 0 & 0.006 & 493 & 84746 & 2.693 & 4.928 & 0.489 \\
\hline Cake & 15 & 2 & 0 & 0.036 & 778 & 21542 & 2.891 & 4.333 & 0.493 \\
\hline Centrate & 15 & 2 & 4500 & 0.002 & 130 & 63524 & 2.114 & 4.803 & 0.438 \\
\hline Cake & 15 & 2 & 4500 & 0.133 & 1987 & 14961 & 3.298 & 4.175 & 0.849 \\
\hline Centrate & 15 & 2 & 3500 & 0.002 & 329 & 135559 & 2.517 & 5.132 & 0.446 \\
\hline Cake & 15 & 2 & 3500 & 0.116 & 1987 & 17178 & 3.298 & 4.235 & 0.849 \\
\hline Centrate & 15 & 2 & 2500 & 0.002 & 107 & 48372 & 2.030 & 4.685 & 0.452 \\
\hline Cake & 15 & 2 & 2500 & 0.107 & 1803 & 16892 & 3.256 & 4.228 & 0.000 \\
\hline
\end{tabular}


Figure 5.4 CA4600 polymer, optimum dose: $15 \mathrm{~g} / \mathrm{kg}$ DS

\begin{tabular}{|c|c|c|c|c|c|c|c|c|c|}
\hline $\begin{array}{c}\text { Sample } \\
\text { type }\end{array}$ & $\begin{array}{c}\text { Polymer } \\
\text { dose (g / } \\
\text { kg DS) }\end{array}$ & Day & $\begin{array}{l}\text { Centrifuge } \\
\text { speed } \\
\text { (rpm) }\end{array}$ & $\begin{array}{l}\text { Total } \\
\text { solids }\end{array}$ & $\begin{array}{c}\text { MPN / g } \\
\text { wet } \\
\text { weight }\end{array}$ & $\begin{array}{c}\text { MPN/ g } \\
\text { DW }\end{array}$ & $\begin{array}{c}\text { log (MPN } \\
\text { / g wet } \\
\text { weight) }\end{array}$ & $\begin{array}{c}\log \\
(\mathrm{MPN} / \mathrm{g} \\
\text { DW) }\end{array}$ & $\begin{array}{c}95 \% \log \\
\text { MPN }\end{array}$ \\
\hline Digested & 0 & $\overline{0}$ & 0 & 0.026 & $\overline{4447}$ & 16917 & 2.650 & 4.228 & 0.600 \\
\hline Centrate & 0 & 0 & 4500 & 0.004 & 78 & 21011 & 1.891 & 4.322 & 0.493 \\
\hline Sludge & 0 & 0 & 4500 & 0.133 & 2312 & 17432 & 3.364 & 4.241 & 0.421 \\
\hline Centrate & 5 & 0 & 0 & 0.013 & 1724 & 131067 & 3.237 & 5.117 & 0.425 \\
\hline Sludge & 5 & 0 & 0 & 0.029 & 22120 & 772625 & 4.345 & 5.888 & 0.426 \\
\hline Centrate & 10 & 0 & 0 & 0.003 & 231 & 67618 & 2.364 & 4.830 & 0.421 \\
\hline Sludge & 10 & 0 & 0 & 0.039 & 2312 & 59534 & 3.364 & 4.775 & 0.421 \\
\hline Centrate & 15 & 0 & 0 & 0.001 & 456 & 370612 & 2.659 & 5.569 & 0.482 \\
\hline Sludge & 15 & 0 & 0 & 0.083 & 2312 & 27996 & 3.364 & 4.447 & 0.421 \\
\hline Centrate & 20 & 0 & 0 & 0.002 & 107 & 56006 & 2.030 & 4.748 & 0.452 \\
\hline Sludge & 20 & 0 & 0 & 0.054 & 3291 & 60882 & 3.517 & 4.784 & 0.446 \\
\hline Centrate & 25 & 0 & 0 & 0.002 & 18 & 9090 & 1.256 & 3.959 & \#NUM! \\
\hline Sludge & 25 & 0 & 0 & 0.050 & 2161 & 42860 & 3.335 & 4.632 & 0.420 \\
\hline Centrate & 5 & 0 & 4500 & 0.004 & 169 & 46799 & 2.228 & 4.670 & 0.421 \\
\hline Sludge & 5 & 0 & 4500 & 0.129 & 4932 & 38112 & 3.693 & 4.581 & 0.489 \\
\hline Centrate & 10 & 0 & 4500 & 0.002 & 78 & 33835 & 1.891 & 4.529 & 0.493 \\
\hline Sludge & 10 & 0 & 4500 & 0.143 & 4932 & 34562 & 3.693 & 4.539 & 0.489 \\
\hline Centrate & 15 & 0 & 4500 & 0.001 & 20 & 16439 & 1.298 & 4.216 & 0.849 \\
\hline Sludge & 15 & 0 & 4500 & 0.139 & 10860 & 77957 & 4.036 & 4.892 & 0.453 \\
\hline Centrate & 20 & 0 & 4500 & 0.002 & 18 & 11887 & 1.256 & 4.075 & \#NUM! \\
\hline Sludge & 20 & 0 & 4500 & 0.138 & 7924 & 57474 & 3.899 & 4.759 & 0.484 \\
\hline Centrate & 25 & 0 & 4500 & 0.002 & 18 & 11307 & 1.256 & 4.053 & \#NUM! \\
\hline Sludge & 25 & 0 & 4500 & 0.133 & 2312 & 17404 & 3.364 & 4.241 & 0.421 \\
\hline
\end{tabular}


Figure 5.4 CA4600 polymer, optimum dose: $15 \mathrm{~g} / \mathrm{kg}$ DS Continued from previous page

\begin{tabular}{|c|c|c|c|c|c|c|c|c|c|}
\hline $\begin{array}{c}\text { Sample } \\
\text { type }\end{array}$ & $\begin{array}{l}\text { Polymer } \\
\text { dose (g I } \\
\text { kg DS) }\end{array}$ & Day & $\begin{array}{c}\text { Centrifuge } \\
\text { speed } \\
(\mathrm{rpm})\end{array}$ & $\begin{array}{l}\text { Total } \\
\text { solids }\end{array}$ & $\begin{array}{c}\text { MPN / g } \\
\text { wet } \\
\text { weight }\end{array}$ & $\begin{array}{c}\text { MPN/ } \mathbf{g} \\
\text { DW }\end{array}$ & $\begin{array}{l}\log \text { (MPN } \\
\text { / g wet } \\
\text { weight) }\end{array}$ & $\begin{array}{c}\log \\
(\mathrm{MPN} / \mathrm{g} \\
\text { DW) }\end{array}$ & $\begin{array}{c}95 \% \log \\
\text { MPN }\end{array}$ \\
\hline Supernatal & $\overline{0}$ & 2 & 0 & 0.005 & 169 & 34572 & 2.228 & 4.539 & 0.421 \\
\hline Sludge cal & 0 & 2 & 0 & 0.052 & 2312 & 44658 & 3.364 & 4.650 & 0.421 \\
\hline Centrate & 0 & 2 & 4500 & 0.004 & 20 & 5497 & 1.298 & 3.740 & 0.849 \\
\hline Sludge & 0 & 2 & 4500 & 0.128 & 2312 & 18092 & 3.364 & 4.257 & 0.421 \\
\hline Centrate & 5 & 2 & 0 & 0.004 & 20 & 5618 & 1.298 & 3.750 & 0.849 \\
\hline Sludge & 5 & 2 & 0 & 0.044 & 1276 & 28821 & 3.106 & 4.460 & 0.436 \\
\hline Centrate & 10 & 2 & 0 & 0.003 & 231 & 70160 & 2.364 & 4.846 & 0.421 \\
\hline Sludge & 10 & 2 & 0 & 0.041 & 1276 & 31129 & 3.106 & 4.493 & 0.436 \\
\hline Centrate & 15 & 2 & 0 & 0.002 & 18 & 7761 & 1.256 & 3.890 & \#NUM! \\
\hline Sludge & 15 & 2 & 0 & 0.078 & 3291 & 42109 & 3.517 & 4.624 & 0.446 \\
\hline Centrate & 20 & 2 & 0 & 0.002 & 45 & 21620 & 1.650 & 4.335 & 0.600 \\
\hline Sludge & 20 & 2 & 0 & 0.076 & 2312 & 30495 & 3.364 & 4.484 & 0.421 \\
\hline Centrate & 25 & 2 & 0 & 0.002 & 169 & 74229 & 2.228 & 4.871 & 0.421 \\
\hline Sludge & 25 & 2 & 0 & 0.071 & 2312 & 32532 & 3.364 & 4.512 & 0.421 \\
\hline Centrate & 5 & 2 & 4500 & 0.002 & 45 & 21901 & 1.650 & 4.340 & 0.600 \\
\hline Sludge & 5 & 2 & 4500 & 0.144 & 2312 & 16079 & 3.364 & 4.206 & 0.421 \\
\hline Centrate & 10 & 2 & 4500 & 0.002 & 78 & 41742 & 1.891 & 4.621 & 0.493 \\
\hline Sludge & 10 & 2 & 4500 & 0.144 & 2312 & 16074 & 3.364 & 4.206 & 0.421 \\
\hline Centrate & 15 & 2 & 4500 & 0.001 & 18 & 13836 & 1.256 & 4.141 & \#NUM! \\
\hline Sludge & 15 & 2 & 4500 & 0.148 & 1276 & 8620 & 3.106 & 3.936 & 0.436 \\
\hline Centrate & 20 & 2 & 4500 & 0.001 & 20 & 15864 & 1.298 & 4.200 & 0.849 \\
\hline Sludge & 20 & 2 & 4500 & 0.135 & 2312 & 17182 & 3.364 & 4.235 & 0.421 \\
\hline Centrate & 25 & 2 & 4500 & 0.001 & 18 & 13219 & 1.256 & 4.121 & \#NUM! \\
\hline
\end{tabular}




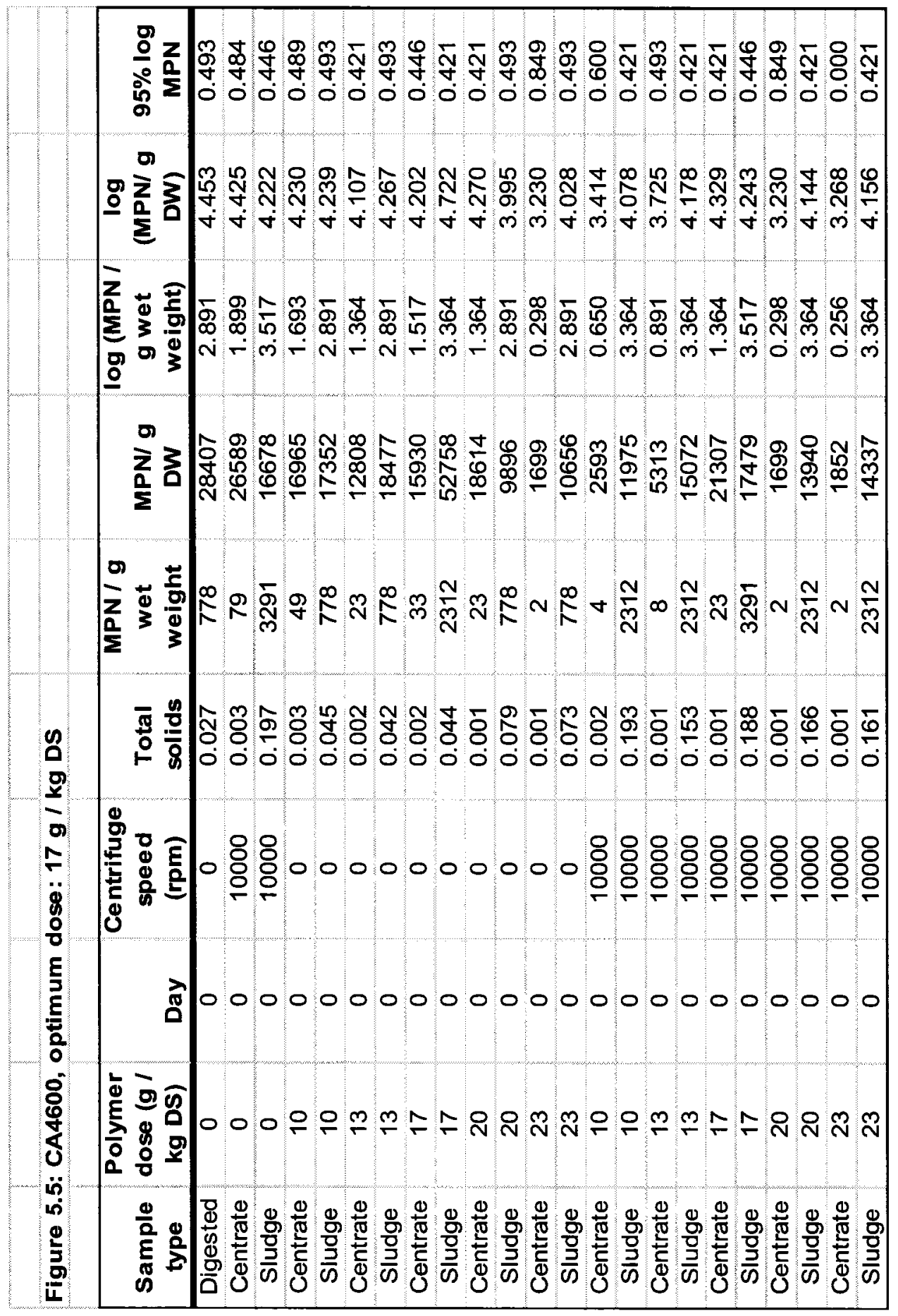


Figure 5.5: CA4600, optimum dose: $17 \mathrm{~g} / \mathrm{kg}$ DS Continued from previous page

\begin{tabular}{|c|c|c|c|c|c|c|c|c|c|}
\hline $\begin{array}{c}\text { Sample } \\
\text { type }\end{array}$ & $\begin{array}{l}\text { Polymer } \\
\text { dose (g / } \\
\text { kg DS) }\end{array}$ & Day & $\begin{array}{c}\text { Centrifuge } \\
\text { speed } \\
\text { (rpm) }\end{array}$ & $\begin{array}{l}\text { Total } \\
\text { solids }\end{array}$ & $\begin{array}{c}\text { MPN/g } \\
\text { wet } \\
\text { weight }\end{array}$ & $\begin{array}{c}\text { MPN/ } \mathbf{g} \\
\text { DW }\end{array}$ & $\begin{array}{c}\log \text { (MPN / } \\
\text { g wet } \\
\text { weight) }\end{array}$ & $\begin{array}{c}\log \\
(\mathrm{MPN} / \mathrm{g} \\
\text { DW) }\end{array}$ & $\begin{array}{c}95 \% \log \\
\text { MPN }\end{array}$ \\
\hline Supernatan & 0 & 2 & 0 & 0.004 & 35 & 7899 & 1.541 & 3.898 & 0.463 \\
\hline Sludge cakı & 0 & 2 & 0 & 0.046 & 199 & 4360 & 2.298 & 3.639 & 0.849 \\
\hline Centrate & 0 & 2 & 10000 & 0.004 & 17 & 4459 & 1.228 & 3.649 & 0.421 \\
\hline Sludge & 0 & 2 & 10000 & 0.139 & 3291 & 23650 & 3.517 & 4.374 & 0.446 \\
\hline Centrate & 10 & 2 & 0 & 0.003 & 23 & 8506 & 1.364 & 3.930 & 0.421 \\
\hline Sludge & 10 & 2 & 0 & 0.042 & 180 & 4338 & 2.256 & 3.637 & 0.000 \\
\hline Centrate & 13 & 2 & 0 & 0.002 & 49 & 23686 & 1.693 & 4.374 & 0.489 \\
\hline Sludge & 13 & 2 & 0 & 0.036 & 180 & 4940 & 2.256 & 3.694 & 0.000 \\
\hline Centrate & 17 & 2 & 0 & 0.002 & 130 & 52649 & 2.114 & 4.721 & 0.438 \\
\hline Sludge & 17 & 2 & 0 & 0.038 & 2312 & 60738 & 3.364 & 4.783 & 0.421 \\
\hline Centrate & 20 & 2 & 0 & 0.001 & 23 & 16583 & 1.364 & 4.220 & 0.421 \\
\hline Sludge & 20 & 2 & 0 & 0.069 & 180 & 2626 & 2.256 & 3.419 & 0.000 \\
\hline Centrate & 23 & 2 & 0 & 0.001 & 17 & 12336 & 1.228 & 4.091 & 0.421 \\
\hline Sludge & 23 & 2 & 0 & 0.071 & 447 & 6309 & 2.650 & 3.800 & 0.600 \\
\hline Centrate & 10 & 2 & 10000 & 0.002 & 8 & 4458 & 0.891 & 3.649 & 0.493 \\
\hline Sludge & 10 & 2 & 10000 & 0.176 & 3291 & 18679 & 3.517 & 4.271 & 0.446 \\
\hline Centrate & 13 & 2 & 10000 & 0.001 & 23 & 16775 & 1.364 & 4.225 & 0.421 \\
\hline Sludge & 13 & 2 & 10000 & 0.186 & 3291 & 17666 & 3.517 & 4.247 & 0.446 \\
\hline Centrate & 17 & 2 & 10000 & 0.001 & 11 & 9287 & 1.030 & 3.968 & 0.452 \\
\hline Sludge & 17 & 2 & 10000 & 0.171 & 17240 & 101040 & 4.237 & 5.004 & 0.425 \\
\hline Centrate & 20 & 2 & 10000 & 0.001 & 2 & 1623 & 0.298 & 3.210 & 0.849 \\
\hline Sludge & 20 & 2 & 10000 & 0.164 & 23980 & 146330 & 4.380 & 5.165 & 0.429 \\
\hline Centrate & 23 & 2 & 10000 & 0.001 & 2 & 1541 & 0.256 & 3.188 & 0.000 \\
\hline
\end{tabular}




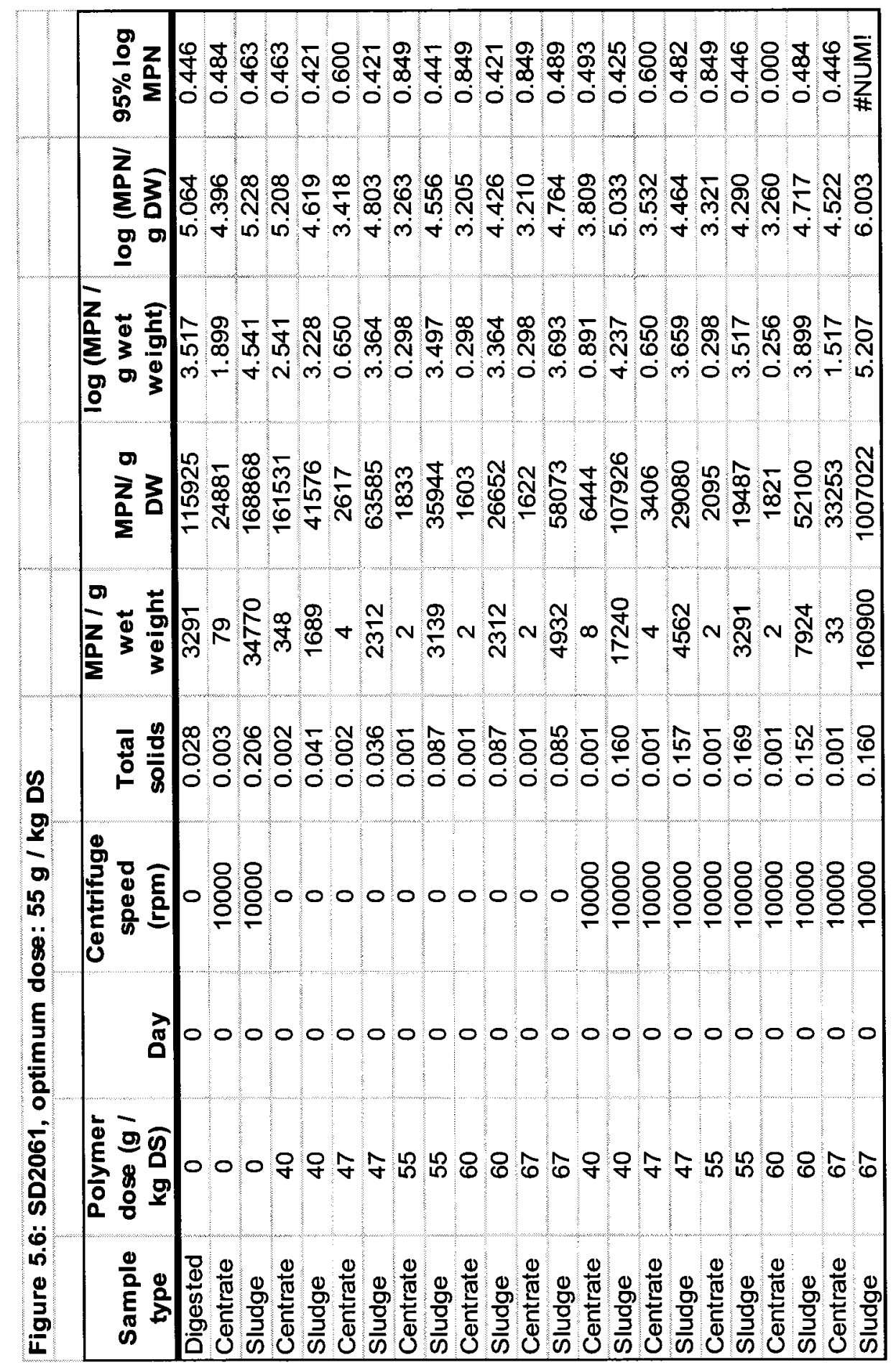


Figure 5.6: SD2061, optimum dose: $55 \mathrm{~g} / \mathrm{kg}$ DS Continued from previous page

\begin{tabular}{|c|c|c|c|c|c|c|c|c|c|}
\hline $\begin{array}{c}\text { Sample } \\
\text { type }\end{array}$ & $\begin{array}{c}\text { Polymer } \\
\text { dose (g / } \\
\text { kg DS) }\end{array}$ & Day & $\begin{array}{c}\text { Centrifuge } \\
\text { speed } \\
\text { (rpm) }\end{array}$ & $\begin{array}{l}\text { Total } \\
\text { solids }\end{array}$ & $\begin{array}{c}\text { MPN / g } \\
\text { wet } \\
\text { weight }\end{array}$ & $\begin{array}{c}\text { MPN/ g } \\
\text { DW }\end{array}$ & $\begin{array}{c}\text { Iog (MPN } / \\
\text { g wet } \\
\text { weight) }\end{array}$ & $\begin{array}{c}\log (\mathrm{MPN} / \\
\mathrm{g} \mathrm{DW})\end{array}$ & $\begin{array}{c}95 \% \log \\
\text { MPN }\end{array}$ \\
\hline Supernatan & $\overline{0}$ & $\overline{2}$ & $\overline{0}$ & 0.009 & 240 & 25442 & 2.380 & 4.406 & 0.429 \\
\hline Sludge cak & 0 & 2 & 0 & 0.043 & 180 & 4213 & 2.256 & 3.625 & 0.000 \\
\hline Centrate & 0 & 2 & 10000 & 0.004 & 13 & 3600 & 1.106 & 3.556 & 0.436 \\
\hline Sludge & 0 & 2 & 10000 & 0.176 & 2312 & 13113 & 3.364 & 4.118 & 0.421 \\
\hline Centrate & 40 & 2 & 0 & 0.002 & 49 & 22955 & 1.693 & 4.361 & 0.489 \\
\hline Sludge & 40 & 2 & 0 & 0.038 & 180 & 4685 & 2.256 & 3.671 & 0.000 \\
\hline Centrate & 47 & 2 & 0 & 0.003 & 240 & 85189 & 2.380 & 4.930 & 0.429 \\
\hline Sludge & 47 & 2 & 0 & 0.051 & 447 & 8836 & 2.650 & 3.946 & 0.600 \\
\hline Centrate & 55 & 2 & 0 & 0.002 & 23 & 12374 & 1.364 & 4.093 & 0.421 \\
\hline Sludge & 55 & 2 & 0 & 0.085 & 2312 & 27104 & 3.364 & 4.433 & 0.421 \\
\hline Centrate & 60 & 2 & 0 & 0.002 & 13 & 7084 & 1.106 & 3.850 & 0.436 \\
\hline Sludge & 60 & 2 & 0 & 0.085 & 2116 & 24913 & 3.326 & 4.396 & 0.419 \\
\hline Centrate & 67 & 2 & 0 & 0.002 & 49 & 23297 & 1.693 & 4.367 & 0.489 \\
\hline Sludge & 67 & 2 & 0 & 0.081 & 1384 & 16983 & 3.141 & 4.230 & 0.430 \\
\hline Centrate & 40 & 2 & 10000 & 0.002 & 79 & 38688 & 1.899 & 4.588 & 0.484 \\
\hline Sludge & 40 & 2 & 10000 & 0.161 & 2312 & 14376 & 3.364 & 4.158 & 0.421 \\
\hline Centrate & 47 & 2 & 10000 & 0.001 & 8 & 7783 & 0.891 & 3.891 & 0.493 \\
\hline Sludge & 47 & 2 & 10000 & 0.164 & 3291 & 20017 & 3.517 & 4.301 & 0.446 \\
\hline Centrate & 55 & 2 & 10000 & 0.001 & 2 & 2131 & 0.298 & 3.329 & 0.849 \\
\hline Sludge & 55 & 2 & 10000 & 0.172 & 7924 & 46133 & 3.899 & 4.664 & 0.484 \\
\hline Centrate & 60 & 2 & 10000 & 0.001 & 2 & 2312 & 0.298 & 3.364 & 0.849 \\
\hline Sludge & 60 & 2 & 10000 & 0.156 & 34770 & 223231 & 4.541 & 5.349 & 0.463 \\
\hline Centrate & 67 & 2 & 10000 & 0.001 & 2 & 2133 & 0.260 & 3.329 & 0.884 \\
\hline Sludge & 67 & 2 & 10000 & 0.166 & 23980 & 144891 & 4.380 & 5.161 & 0.429 \\
\hline
\end{tabular}


Sample ANOVA calculation

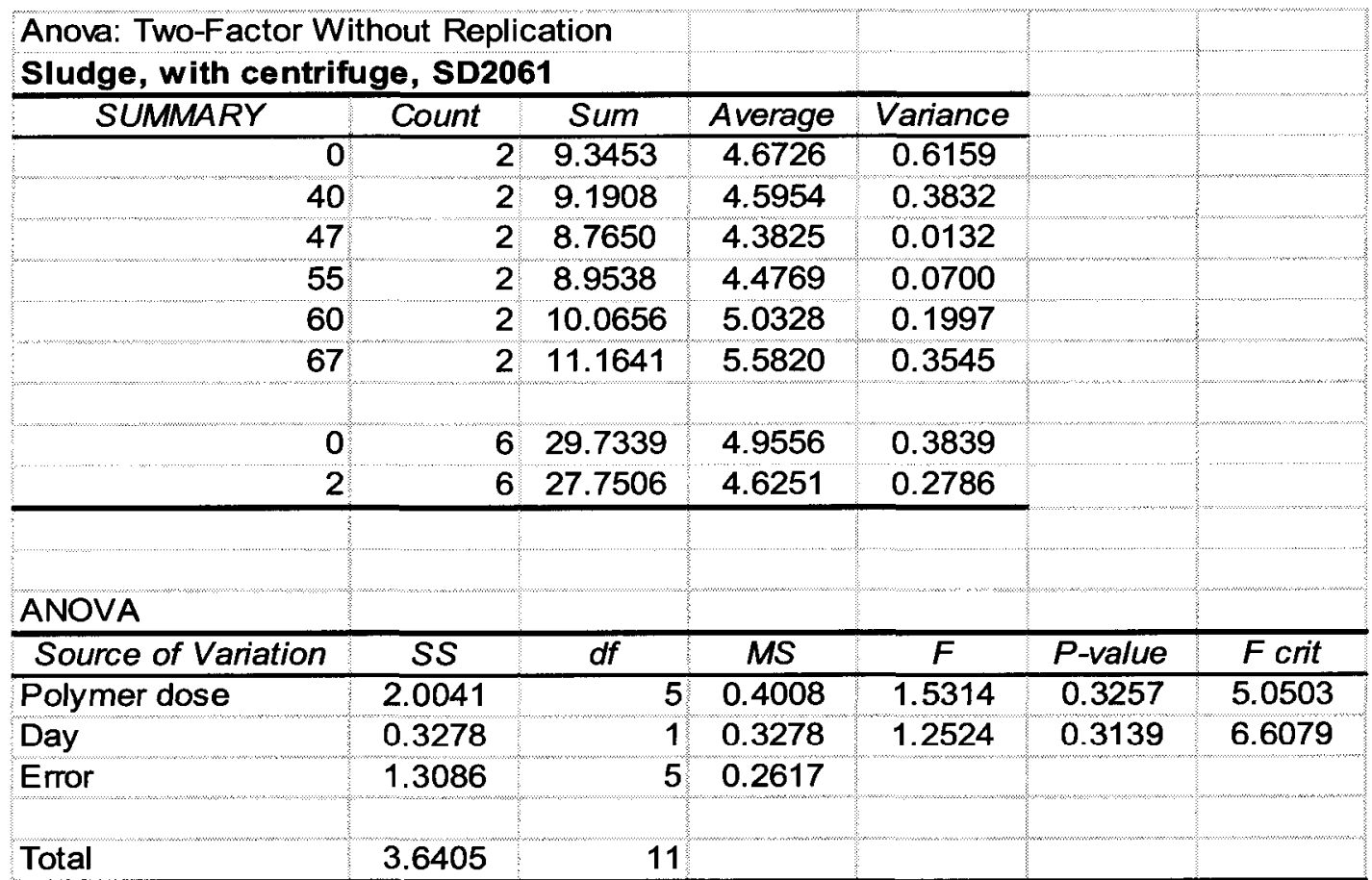




\begin{tabular}{|c|c|c|c|c|c|c|c|c|c|}
\hline \multicolumn{9}{|c|}{ Figure 5.7 and 5.9: Full-scale Treatment Plant $A$ and aging } & \\
\hline \multirow{2}{*}{\multicolumn{9}{|c|}{ Run \#1 }} & \\
\hline & & & & & & & & & \\
\hline Sample type & $\begin{array}{c}\text { Temper- } \\
\text { ature } \\
\left({ }^{\circ} \mathrm{C}\right)\end{array}$ & Day & $\begin{array}{c}\text { Solids } \\
(\%)\end{array}$ & $\begin{array}{c}\text { MPN / g } \\
\text { wet } \\
\text { weight }\end{array}$ & $\begin{array}{c}\text { MPN / g } \\
\text { DW }\end{array}$ & $\begin{array}{l}\log \text { (MPN } \\
\text { / g wet } \\
\text { weight) }\end{array}$ & $\begin{array}{c}\log (M P N) \\
\text { g DW) }\end{array}$ & $95 \% \mathrm{Cl}$ & \\
\hline Digester in & NA & 0 & 4.11 & $1.81 \mathrm{E}+06$ & $4.19 E+07$ & 6.258 & 7.622 & 0.429 & \\
\hline Digested & NA & 0 & 2.75 & $2.13 E+03$ & $8.04 E+04$ & 3.328 & 4.905 & 0.420 & \\
\hline Post polymer & NA & 0 & 2.31 & $1.36 E+03$ & $5.80 E+04$ & 3.133 & 4.764 & 0.457 & \\
\hline Centrate & NA & 0 & 0.22 & $4.47 E+02$ & $1.99 \mathrm{E}+05$ & 2.650 & 5.300 & 0.467 & \\
\hline Cake & NA & 0 & 31.25 & $1.81 E+04$ & $5.77 E+04$ & 4.257 & 4.761 & 0.000 & \\
\hline Cake & 24 & 7 & 30.89 & $1.50 E+06$ & $4.87 E+06$ & 6.177 & 6.687 & 0.000 & \\
\hline Cake & 4 & 7 & 31.10 & $1.59 E+03$ & $5.11 E+03$ & 3.202 & 3.709 & 0.429 & \\
\hline Cake & 4 and 24 & 14 & 30.53 & $8.70 \mathrm{E}+05$ & $2.85 E+06$ & 5.940 & 6.455 & 0.454 & \\
\hline \multicolumn{9}{|l|}{ Run \#2 } & \\
\hline Sample type & $\begin{array}{l}\text { Temper- } \\
\text { ature } \\
\left({ }^{\circ} \mathrm{C}\right)\end{array}$ & Day & $\begin{array}{c}\text { Solids } \\
(\%)\end{array}$ & $\begin{array}{c}\text { MPN / g } \\
\text { wet } \\
\text { weight }\end{array}$ & $\begin{array}{c}\text { MPN / g } \\
\text { DW }\end{array}$ & $\begin{array}{c}\text { log (MPN } \\
\text { / g wet } \\
\text { weight) }\end{array}$ & $\begin{array}{c}\log (\text { MPN } / \\
\text { g DW) }\end{array}$ & $95 \% \mathrm{Cl}$ & \\
\hline Digester In & NA & 0 & 4.72 & $1.71 E+06$ & $3.63 E+07$ & 6.234 & 7.560 & 0.425 & \\
\hline Digested & NA & 0 & 2.60 & $1.92 E+03$ & $7.38 E+04$ & 3.284 & 4.868 & 0.434 & \\
\hline Post Polymer & NA & 0 & 2.31 & $1.38 E+03$ & $5.96 E+04$ & 3.140 & 4.775 & 0.447 & \\
\hline Centrate & NA & 0 & 0.16 & $7.29 E+02$ & $4.52 E+05$ & 2.863 & 5.655 & 0.490 & \\
\hline Cake & NA & 0 & 33.31 & $1.20 \mathrm{E}+05$ & $3.60 E+05$ & 5.079 & 5.557 & 0.438 & \\
\hline
\end{tabular}


Figure 5.8 and 5.10: Full-scale Treatment Plant $B$ and aging

\begin{tabular}{|c|c|c|c|c|c|c|c|c|}
\hline Sample type & $\begin{array}{c}\text { Temper- } \\
\text { ature } \\
\left({ }^{\circ} \mathrm{C}\right)\end{array}$ & Day & $\begin{array}{c}\text { Solids } \\
\text { (\%) }\end{array}$ & $\begin{array}{c}\text { MPN / g } \\
\text { wet } \\
\text { weight } \\
\end{array}$ & $\begin{array}{c}\text { MPN / } \mathbf{g} \\
\text { DW }\end{array}$ & $\begin{array}{l}\text { log (MPN } \\
\text { / g wet } \\
\text { weight) }\end{array}$ & $\begin{array}{c}\log (\mathrm{MPN} / \\
\mathrm{g} \text { DW) }\end{array}$ & $95 \% \mathrm{Cl}$ \\
\hline Digester In & NA & 0 & 3.30 & $3.62 E+05$ & $1.10 \mathrm{E}+07$ & 5.558 & 7.040 & 0.454 \\
\hline Digested & NA & 0 & 1.80 & $2.60 \mathrm{E}+03$ & $1.45 E+05$ & 3.415 & 5.160 & 0.430 \\
\hline Post Polymer & NA & 0 & 1.53 & $3.28 E+03$ & $2.14 \mathrm{E}+05$ & 3.516 & 5.331 & 0.451 \\
\hline Centrate & NA & 0 & 0.06 & $4.43 E+02$ & $7.20 \mathrm{E}+05$ & 2.647 & 5.858 & 0.458 \\
\hline Cake & NA & 0 & 18.26 & $9.34 E+04$ & $5.12 E+05$ & 4.971 & 5.709 & 0.469 \\
\hline Pellets & NA & 0 & 94.17 & 1.86E+01 & $1.98 \mathrm{E}+01$ & 1.270 & 1.296 & 0.849 \\
\hline Cake & 24 & 4 & 16.71 & $5.63 E+04$ & $3.37 E+05$ & 4.750 & 5.527 & 0.482 \\
\hline Cake & 4 & 4 & 18.48 & $7.79 \mathrm{E}+04$ & $4.22 \mathrm{E}+05$ & 4.892 & 5.625 & 0.519 \\
\hline Pellets & 24 & 4 & 98.26 & $1.86 \mathrm{E}+01$ & $1.90 \mathrm{E}+01$ & 1.270 & 1.278 & 0.849 \\
\hline
\end{tabular}




\begin{tabular}{|c|c|c|c|c|c|c|}
\hline & & & & & & \\
\hline & & & & & & \\
\hline \multicolumn{7}{|c|}{ Figure 5.11: Treatment Plant A: Full-scale, aging } \\
\hline & & & & & & \\
\hline Sample type & Temperature & Day & Solids \% & $\begin{array}{l}\log (M P N / g \\
\text { wet weight) }\end{array}$ & $\begin{array}{c}\log (\mathrm{MPN} / \mathrm{g} \\
\text { DW) }\end{array}$ & $95 \% \mathrm{Cl}$ \\
\hline Digester in & NA & 0 & 4.130 & 4.256 & 5.640 & 0.000 \\
\hline Digested & NA & 0 & 2.698 & 2.256 & 3.825 & 0.000 \\
\hline Cake & NA & 0 & 37.938 & 3.845 & 4.266 & 0.493 \\
\hline Centrate & NA & 0 & 0.239 & 2.899 & 5.520 & 0.484 \\
\hline Cake + PBS & NA & 0 & 3.794 & 3.845 & 4.266 & 0.493 \\
\hline Cake + Centrate & NA & 0 & 2.195 & 3.517 & 5.176 & 0.446 \\
\hline Digested & Refrigerator & 2 & 2.673 & 2.693 & 4.266 & 0.489 \\
\hline Cake & Refrigerator & 2 & 36.397 & 3.899 & 4.338 & 0.484 \\
\hline Centrate & Refrigerator & 2 & 0.206 & 2.364 & 5.049 & 0.421 \\
\hline Cake + PBS & Refrigerator & 2 & 3.589 & 4.207 & 5.652 & \#NUM! \\
\hline Cake + Centrate & Refrigerator & 2 & 1.958 & 3.963 & 5.671 & 0.505 \\
\hline Digested & Room temperature & 2 & 2.626 & 2.693 & 4.274 & 0.489 \\
\hline Cake & Room temperature & 2 & 35.239 & 6.207 & 6.660 & \#NUM! \\
\hline Centrate & Room temperature & 2 & 0.221 & 3.345 & 6.001 & 0.426 \\
\hline Cake + PBS & Room temperature & 2 & 3.683 & 6.207 & 7.640 & \#NUM! \\
\hline Cake + Centrate & Room temperature & 2 & 1.984 & 5.207 & 6.909 & 0.473 \\
\hline Digested & Incubator & 2 & 2.603 & 2.306 & 3.890 & 0.419 \\
\hline Cake & Incubator & 2 & 34.311 & 7.207 & 8.671 & \#NUM! \\
\hline Centrate & Incubator & 2 & 0.169 & 2.517 & 5.288 & 0.446 \\
\hline Cake + PBS & Incubator & 2 & 3.640 & 6.207 & 7.645 & \#NUM! \\
\hline Cake + Centrate & Incubator & 2 & 1.949 & 5.148 & 6.858 & 0.433 \\
\hline
\end{tabular}


Figure 5.11: Treatment Plant A: Full-scale, aging Continued from previous page

\begin{tabular}{|l|l|c|c|c|c|c|}
\hline Sample type & Temperature & Day & Solids \% & $\begin{array}{c}\text { Iog (MPN/g } \\
\text { wet weight) }\end{array}$ & $\begin{array}{c}\text { Iog (MPN/ g } \\
\text { DW) }\end{array}$ & 95\% Cl \\
\hline Digested & Refrigerator & 4 & 2.708 & 3.541 & 5.109 & 0.463 \\
\hline Cake & Refrigerator & 4 & 35.818 & 4.237 & 4.682 & 0.425 \\
\hline Centrate & Refrigerator & 4 & 0.241 & 1.969 & 4.587 & 0.468 \\
\hline Cake + PBS & Refrigerator & 4 & 3.723 & 2.256 & 3.685 & 0.000 \\
\hline Cake + Centrate & Refrigerator & 4 & 1.986 & 2.256 & 3.958 & 0.000 \\
\hline Digested & Room temperature & 4 & 2.599 & 1.256 & 2.841 & 0.000 \\
\hline Cake & Room temperature & 4 & 34.081 & 6.036 & 6.503 & 0.453 \\
\hline Centrate & Room temperature & 4 & 0.185 & 1.260 & 3.993 & 0.884 \\
Cake + PBS & Room temperature & 4 & 3.660 & 5.541 & 6.978 & 0.463 \\
\hline Cake + Centrate & Room temperature & 4 & 1.941 & 4.141 & 5.853 & 0.430 \\
\hline Digested & Incubator & 4 & 2.551 & 2.693 & 4.286 & 0.489 \\
\hline Cake & Incubator & 4 & 27.134 & 7.207 & 7.773 & 0.473 \\
Centrate & Incubator & 4 & 0.222 & 1.298 & 3.951 & 0.849 \\
\hline Cake + PBS & Incubator & 4 & 3.075 & 5.899 & 7.411 & 0.505 \\
\hline Cake + Centrate & Incubator & 4 & 2.403 & 3.891 & 5.510 & 0.493 \\
\hline
\end{tabular}


Figure 5.12: Treatment Plant A: Lab-scale, aging

\begin{tabular}{|c|c|c|c|c|c|c|}
\hline Sample type & Temperature & Day & Solids \% & $\begin{array}{l}\text { Tog (MPN / g } \\
\text { wet weight) }\end{array}$ & $\begin{array}{c}\log (M P N / g \\
\text { DW) }\end{array}$ & $95 \% \mathrm{Cl}$ \\
\hline Digester in & NA & 0 & 4.790 & 6.237 & 7.556 & 0.425 \\
\hline Digested & NA & 0 & 2.680 & 3.114 & 4.685 & 0.438 \\
\hline Cake & NA & 0 & 17.699 & 4.541 & 5.293 & 0.463 \\
\hline Centrate & NA & 0 & 0.272 & 1.298 & 3.863 & 0.849 \\
\hline Cake + PBS & NA & 0 & 1.770 & 4.541 & 5.293 & 0.463 \\
\hline Cake + Centrate & NA & 0 & 0.934 & 2.693 & 4.723 & 0.489 \\
\hline Digested & Refrigerator & 2 & 2.733 & 3.237 & 4.800 & 0.425 \\
\hline Cake & Refrigerator & 2 & 17.979 & 2.845 & 4.590 & 0.493 \\
\hline Centrate & Refrigerator & 2 & 0.290 & 1.106 & 3.644 & 0.436 \\
\hline Cake + PBS & Refrigerator & 2 & 1.745 & 3.106 & 4.864 & 0.436 \\
\hline Cake + Centrate & Refrigerator & 2 & 1.121 & 3.380 & 5.330 & 0.429 \\
\hline Digested & Room temperature & 2 & 2.818 & 3.036 & 4.586 & 0.453 \\
\hline Cake & Room temperature & 2 & 24.873 & 4.659 & 5.263 & 0.446 \\
\hline Centrate & Room temperature & 2 & 0.295 & 1.693 & 4.223 & 0.489 \\
\hline Cake + PBS & Room temperature & 2 & 1.830 & 3.891 & 5.628 & 0.493 \\
\hline Cake + Centrate & Room temperature & 2 & 1.174 & 3.298 & 5.228 & 0.849 \\
\hline Digested & Incubator & 2 & 2.678 & 3.734 & 5.306 & 0.519 \\
\hline Cake & Incubator & 2 & 17.284 & 4.228 & 4.990 & 0.421 \\
\hline Centrate & Incubator & 2 & 0.291 & 2.963 & 5.499 & 0.505 \\
\hline Cake + PBS & Incubator & 2 & 15.606 & 4.256 & 5.063 & 0.849 \\
\hline Cake + Centrate & Incubator & 2 & 1.137 & 3.298 & 5.242 & 0.000 \\
\hline
\end{tabular}


Figure 5.12: Treatment Plant A: Lab-scale, aging Continued from previous page

\begin{tabular}{|c|c|c|c|c|c|c|}
\hline Sample type & Temperature & Day & Solids \% & $\begin{array}{l}\text { log (MPN / g } \\
\text { wet weight) }\end{array}$ & $\begin{array}{c}\log (\mathrm{MPN} / \mathrm{g} \\
\text { DW) }\end{array}$ & $95 \% \mathrm{Cl}$ \\
\hline Digested & Refrigerator & 4 & 2.697 & 2.693 & 4.262 & 0.489 \\
\hline Cake & Refrigerator & 4 & 16.708 & 3.433 & 4.210 & 0.429 \\
\hline Centrate & Refrigerator & 4 & 0.290 & 2.541 & 5.079 & 0.463 \\
\hline Cake + PBS & Refrigerator & 4 & 1.570 & 1.298 & 3.102 & 0.849 \\
\hline Cake + Centrate & Refrigerator & 4 & 1.280 & 3.114 & 5.006 & 0.438 \\
\hline Digested & Room temperature & 4 & 2.713 & 2.517 & 4.084 & 0.446 \\
\hline Cake & Room temperature & 4 & 17.078 & 3.891 & 4.658 & 0.493 \\
\hline Centrate & Room temperature & 4 & 0.278 & 0.256 & 2.813 & 0.000 \\
\hline Cake + PBS & Room temperature & 4 & 1.692 & 3.256 & 5.028 & 0.000 \\
\hline Cake + Centrate & Room temperature & 4 & 1.202 & 2.256 & 4.176 & 0.000 \\
\hline Digested & Incubator & 4 & 2.604 & 1.298 & 2.883 & 0.849 \\
\hline Cake & Incubator & 4 & 17.548 & 3.256 & 4.012 & 0.000 \\
\hline Centrate & Incubator & 4 & 0.268 & 0.256 & 2.828 & 0.000 \\
\hline Cake + PBS & Incubator & 4 & 1.680 & 3.256 & 5.031 & 0.000 \\
\hline Cake + Centrate & Incubator & 4 & 1.239 & 2.650 & 4.557 & 0.600 \\
\hline
\end{tabular}


Figure 5.13: Treatment Plant B: Full-scale, aging

\begin{tabular}{|c|c|c|c|c|c|c|}
\hline Sample type & Temperature & Day & Solids \% & $\begin{array}{l}\log (M P N / g \\
\text { wet weight) }\end{array}$ & $\begin{array}{c}\log (\mathrm{MPN} / \mathrm{g} \\
\text { DW) }\end{array}$ & $95 \% \mathrm{Cl}$ \\
\hline Digester in & NA & $\overline{0}$ & 2.615 & 5.899 & 7.482 & 0.484 \\
\hline Digested & NA & 0 & 2.082 & 3.520 & 5.201 & 0.446 \\
\hline Cake & NA & 0 & 18.887 & 4.135 & 4.859 & 0.431 \\
\hline Centrate & NA & 0 & 0.121 & 2.106 & 5.022 & 0.436 \\
\hline Cake + PBS & NA & 0 & 1.889 & 3.135 & 4.859 & 0.431 \\
\hline Cake + Centrate & NA & 0 & 1.207 & 3.228 & 5.146 & 0.421 \\
\hline Digested & Refrigerator & 2 & 2.164 & 3.517 & 5.182 & 0.446 \\
\hline Cake & Refrigerator & 2 & 22.026 & 4.517 & 5.174 & 0.446 \\
\hline Centrate & Refrigerator & 2 & 0.173 & 2.693 & 5.454 & 0.489 \\
\hline Cake + PBS & Refrigerator & 2 & 2.188 & 4.380 & 6.040 & 0.429 \\
\hline Cake + Centrate & Refrigerator & 2 & 1.180 & 4.036 & 5.964 & 0.453 \\
\hline Digested & Room temperature & 2 & 2.122 & 3.899 & 5.572 & 0.484 \\
\hline Cake & Room temperature & 2 & 23.321 & 6.735 & 7.367 & 0.519 \\
\hline Centrate & Room temperature & 2 & 0.134 & 2.364 & 5.236 & 0.421 \\
\hline Cake + PBS & Room temperature & 2 & 2.161 & 4.237 & 5.902 & 0.425 \\
\hline Cake + Centrate & Room temperature & 2 & 1.151 & 3.845 & 5.784 & 0.493 \\
\hline Digested & Incubator & 2 & 2.188 & 2.256 & 3.916 & 0.000 \\
\hline Cake & Incubator & 2 & 21.665 & 5.693 & 6.357 & 0.489 \\
\hline Centrate & Incubator & 2 & 0.108 & 2.653 & 5.620 & 0.479 \\
\hline Cake + PBS & Incubator & 2 & 2.186 & 3.496 & 5.156 & 0.000 \\
\hline Cake + Centrate & Incubator & 2 & 1.259 & 4.424 & 6.324 & 0.482 \\
\hline
\end{tabular}


Figure 5.13: Treatment Plant B: Full-scale, aging

\begin{tabular}{|c|c|c|c|c|c|c|}
\hline Sample type & Temperature & Day & Solids \% & $\begin{array}{l}\log (\mathrm{MPN} / \mathrm{g} \\
\text { wet weight) }\end{array}$ & $\begin{array}{c}\log (\mathrm{MPN} / \mathrm{g} \\
\mathrm{DW})\end{array}$ & $95 \% \mathrm{Cl}$ \\
\hline Digested & Refrigerator & 4 & 2.048 & 2.892 & 4.580 & 0.493 \\
\hline Cake & Refrigerator & 4 & 20.831 & 5.237 & 5.918 & 0.425 \\
\hline Centrate & Refrigerator & 4 & 0.106 & 2.517 & 5.491 & 0.446 \\
\hline Cake + PBS & Refrigerator & 4 & 1.105 & 3.659 & 5.616 & 0.482 \\
\hline Cake + Centrate & Refrigerator & 4 & 2.108 & 4.237 & 5.913 & 0.425 \\
\hline Digested & Room temperature & 4 & 1.951 & 2.961 & 4.671 & 0.468 \\
\hline Cake & Room temperature & 4 & 20.859 & 5.845 & 6.526 & 0.493 \\
\hline Centrate & Room temperature & 4 & 0.140 & 2.517 & 5.371 & 0.446 \\
\hline Cake + PBS & Room temperature & 4 & 2.275 & 4.514 & 6.157 & 0.421 \\
\hline Cake + Centrate & Room temperature & 4 & 1.085 & 3.371 & 5.336 & 0.489 \\
\hline Digested & Incubator & 4 & 1.997 & 1.297 & 2.997 & 0.849 \\
\hline Cake & Incubator & 4 & 20.484 & 5.335 & 6.023 & 0.420 \\
\hline Centrate & Incubator & 4 & 0.149 & 1.298 & 4.126 & 0.849 \\
\hline Cake + PBS & Incubator & 4 & 2.059 & 3.391 & 5.078 & 0.436 \\
\hline Cake + Centrate & Incubator & 4 & 1.067 & 3.232 & 5.204 & 0.446 \\
\hline
\end{tabular}


Figure 5.14: Treatment Plant B: Lab-scale, aging

\begin{tabular}{|l|l|c|c|c|c|c|}
\hline Sample type & Temperature & Day & Solids \% & $\begin{array}{l}\text { log (MPN / g } \\
\text { wet weight) }\end{array}$ & $\begin{array}{c}\text { Iog (MPN/ g } \\
\text { DW) }\end{array}$ & 95\% CI \\
\hline Digester in & NA & 0 & 3.641 & 5.364 & 6.803 & 0.421 \\
Digested & NA & 0 & 2.366 & 3.693 & 5.319 & 0.489 \\
\hline Cake & NA & 0 & 13.342 & 4.364 & 5.239 & 0.421 \\
\hline Centrate & NA & 0 & 0.166 & 1.650 & 4.429 & 0.600 \\
\hline Cake + PBS & NA & 0 & 1.334 & 4.364 & 5.239 & 0.421 \\
\hline Cake + Centrate & NA & 0 & 0.701 & 3.228 & 5.382 & 0.421 \\
\hline Digested & Refrigerator & 2 & 1.676 & 3.899 & 5.675 & 0.484 \\
\hline Cake & Refrigerator & 2 & 11.717 & 3.256 & 4.187 & 0.000 \\
\hline Centrate & Refrigerator & 2 & 0.158 & 2.517 & 5.319 & 0.446 \\
\hline Cake + PBS & Refrigerator & 2 & 1.268 & 2.298 & 4.195 & 0.849 \\
\hline Cake + Centrate & Refrigerator & 2 & 0.629 & 2.256 & 4.457 & 0.000 \\
\hline Digested & Room temperature & 2 & 1.785 & 2.891 & 4.639 & 0.493 \\
\hline Cake & Room temperature & 2 & 1.225 & 4.693 & 6.605 & 0.489 \\
\hline Centrate & Room temperature & 2 & 1.642 & 2.335 & 4.119 & 0.420 \\
\hline Cake + PBS & Room temperature & 2 & 1.394 & 3.228 & 5.083 & 0.421 \\
\hline Cake + Centrate & Room temperature & 2 & 0.641 & 2.650 & 4.843 & 0.600 \\
\hline Digested & Incubator & 2 & 1.712 & 3.335 & 5.101 & 0.420 \\
\hline Cake & Incubator & 2 & 11.752 & 4.497 & 5.427 & 0.440 \\
\hline Centrate & Incubator & 2 & 0.175 & 2.517 & 5.274 & 0.446 \\
\hline Cake + PBS & Incubator & 2 & 1.273 & 3.228 & 5.123 & 0.421 \\
\hline Cake + Centrate & Incubator & 2 & 0.610 & 2.256 & 4.471 & 0.000 \\
\hline
\end{tabular}


Figure 5.14: Treatment Plant B: Lab-scale, aging Continued from previous page

\begin{tabular}{|c|c|c|c|c|c|c|}
\hline Sample type & Temperature & Day & Solids \% & $\begin{array}{l}\log (\mathrm{MPN} / \mathrm{g} \\
\text { wet weight) }\end{array}$ & $\begin{array}{c}\log (\text { MPN/ g } \\
\text { DW) }\end{array}$ & $95 \% \mathrm{Cl}$ \\
\hline Digested & Refrigerator & 4 & 1.676 & 1.969 & 3.745 & 0.468 \\
\hline Cake & Refrigerator & 4 & 11.717 & 3.141 & 4.072 & 0.430 \\
\hline Centrate & Refrigerator & 4 & 0.158 & 2.106 & 4.908 & 0.436 \\
\hline Cake + PBS & Refrigerator & 4 & 1.268 & 3.541 & 5.438 & 0.463 \\
\hline Cake + Centrate & Refrigerator & 4 & 0.629 & 3.237 & 5.438 & 0.425 \\
\hline Digested & Room temperature & 4 & 1.785 & 1.256 & 3.004 & 0.000 \\
\hline Cake & Room temperature & 4 & 12.248 & 3.835 & 5.747 & 0.514 \\
\hline Centrate & Room temperature & 4 & 0.164 & 1.364 & 4.149 & 0.421 \\
\hline Cake + PBS & Room temperature & 4 & 13.937 & 3.364 & 4.220 & 0.421 \\
\hline Cake + Centrate & Room temperature & 4 & 0.641 & 2.106 & 4.299 & 0.436 \\
\hline Digested & Incubator & 4 & 1.712 & 2.106 & 3.872 & 0.436 \\
\hline Cake & Incubator & 4 & 11.696 & 4.106 & 5.038 & 0.436 \\
\hline Centrate & Incubator & 4 & 0.175 & 1.298 & 4.054 & 0.849 \\
\hline Cake + PBS & Incubator & 4 & 1.275 & 1.783 & 3.678 & 0.535 \\
\hline Cake + Centrate & Incubator & 4 & 0.610 & 2.517 & 4.732 & 0.446 \\
\hline
\end{tabular}


Figure 5.15: Treatment Plant A: Full-scale, aging

\begin{tabular}{|c|c|c|c|c|c|c|}
\hline Sample type & Temperature & Day & Solids \% & $\begin{array}{l}\log (\mathrm{MPN} / \mathrm{g} \\
\text { wet weight) }\end{array}$ & $\begin{array}{c}\log (M P N / \\
g \text { DW) }\end{array}$ & $95 \% \mathrm{Cl}$ \\
\hline Digester in & NA & 0 & 4.847 & 6.237 & 7.551 & 0.425 \\
\hline Digested & NA & 0 & 4.827 & 2.693 & 4.009 & 0.489 \\
\hline Cake & NA & 0 & 33.634 & 4.541 & 5.014 & 0.463 \\
\hline Centrate & NA & 0 & 0.244 & 2.899 & 5.511 & 0.484 \\
\hline Cake + PBS & NA & 0 & 3.363 & 4.541 & 5.014 & 0.463 \\
\hline Cake + Centrate & NA & 0 & 1.922 & 3.541 & 5.257 & 0.463 \\
\hline Digested & Refrigerator & 2 & 2.781 & 2.228 & 3.783 & 0.421 \\
\hline Cake & Refrigerator & 2 & 33.527 & 3.364 & 3.839 & 0.421 \\
\hline Centrate & Refrigerator & 2 & 0.258 & 2.364 & 4.952 & 0.421 \\
\hline Cake + PBS & Refrigerator & 2 & 3.434 & 2.517 & 3.981 & 0.446 \\
\hline Cake + Centrate & Refrigerator & 2 & 1.895 & 2.899 & 4.621 & 0.484 \\
\hline Digested & Room temperature & 2 & 2.671 & 2.517 & 4.091 & 0.446 \\
\hline Cake & Room temperature & 2 & 33.124 & 6.734 & 7.214 & 0.519 \\
\hline Centrate & Room temperature & 2 & 0.244 & 1.891 & 4.503 & 0.493 \\
\hline Cake + PBS & Room temperature & 2 & 3.131 & 3.601 & 5.105 & 0.421 \\
\hline Cake + Centrate & Room temperature & 2 & 1.899 & 3.364 & 5.085 & 0.628 \\
\hline Digested & Incubator & 2 & 2.638 & 2.364 & 3.943 & 0.421 \\
\hline Cake & Incubator & 2 & 31.452 & 5.517 & 6.020 & 0.446 \\
\hline Centrate & Incubator & 2 & 0.212 & 1.298 & 3.972 & 0.849 \\
\hline Cake + PBS & Incubator & 2 & 3.631 & 5.444 & 6.884 & 0.429 \\
\hline Cake + Centrate & Incubator & 2 & 1.756 & 3.256 & 5.011 & \#NUM! \\
\hline
\end{tabular}


Figure 5.15: Treatment Plant A: Full-scale, aging Continued from previous page

\begin{tabular}{|c|c|c|c|c|c|c|}
\hline Sample type & Temperature & Day & Solids \% & $\begin{array}{l}\log (\mathrm{MPN} / \mathrm{g} \\
\text { wet weight) }\end{array}$ & $\begin{array}{c}\log (\mathrm{MPN} / \\
\mathrm{g} \text { DW) }\end{array}$ & $95 \% \mathrm{Cl}$ \\
\hline Digested & Refrigerator & 4 & 2.810 & 2.364 & 3.915 & 0.421 \\
\hline Cake & Refrigerator & 4 & 34.858 & 3.106 & 3.564 & 0.436 \\
\hline Centrate & Refrigerator & 4 & 0.285 & 1.650 & 4.196 & 0.600 \\
\hline Cake + PBS & Refrigerator & 4 & 3.512 & 2.899 & 4.353 & 0.484 \\
\hline Cake + Centrate & Refrigerator & 4 & 1.850 & 2.517 & 4.250 & 0.446 \\
\hline Digested & Room temperature & 4 & 2.727 & 2.693 & 4.257 & 0.489 \\
\hline Cake & Room temperature & 4 & 34.622 & 5.364 & 5.825 & 0.421 \\
\hline Centrate & Room temperature & 4 & 0.244 & 1.256 & 3.868 & \#NUM! \\
\hline Cake + PBS & Room temperature & 4 & 3.187 & 4.364 & 5.861 & 0.421 \\
\hline Cake + Centrate & Room temperature & 4 & 1.887 & 3.364 & 5.088 & 0.421 \\
\hline Digested & Incubator & 4 & 2.494 & 2.106 & 3.709 & 0.436 \\
\hline Cake & Incubator & 4 & 29.851 & 4.256 & 4.781 & \#NUM! \\
\hline Centrate & Incubator & 4 & 0.235 & 1.256 & 3.884 & \#NUM! \\
\hline Cake + PBS & Incubator & 4 & 3.175 & 4.899 & 6.397 & 0.484 \\
\hline Cake + Centrate & Incubator & 4 & 1.714 & 2.256 & 4.022 & \#NUM! \\
\hline
\end{tabular}




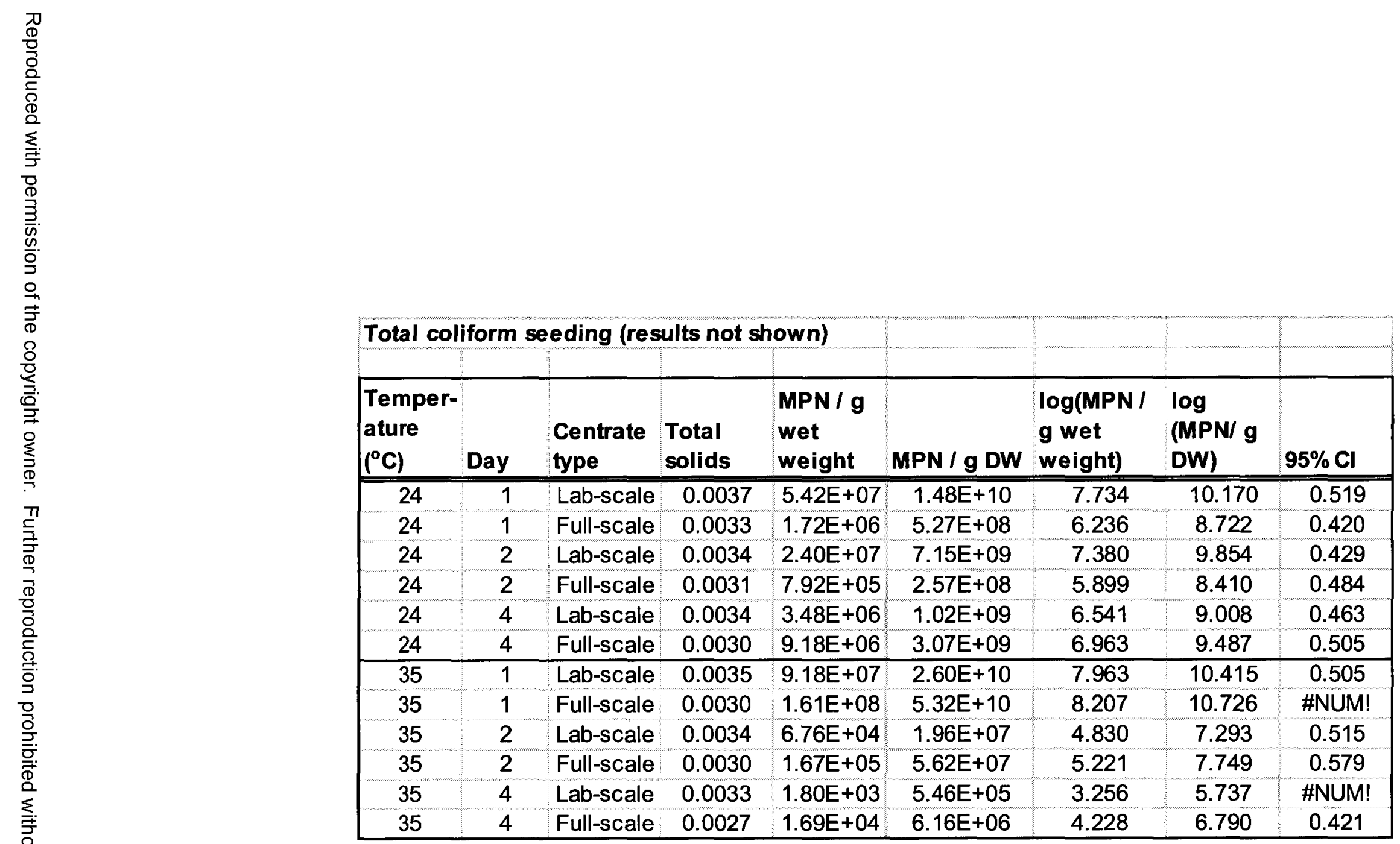




\begin{tabular}{|c|c|c|c|c|c|c|c|c|c|c|c|c|c|c|c|c|c|c|c|c|c|}
\hline & $\begin{array}{l}\bar{u} \\
\stackrel{\circ}{\circ} \\
\delta\end{array}$ & $\sum_{z} \sum^{2}$ & $\sum^{2}$ & : & 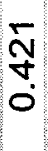 & $\sum_{3}$ & $\begin{array}{c}\mathcal{N} \\
+ \\
0 \\
0\end{array}$ & $\frac{5}{2}$ & Z & & $\bar{v}$ & 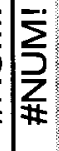 & & $\sum_{i}^{\infty}$ & $\begin{array}{c}\bar{N} \\
\dot{0} \\
\vdots\end{array}$ & $\stackrel{3}{Z}$ & 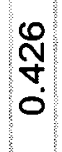 & $\frac{9}{6}:$ & & $\begin{array}{l} \\
v \\
y \\
\vdots\end{array}$ & 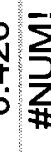 \\
\hline & $\begin{array}{ll}\bar{z} & \\
0 & \xi \\
\Sigma & 0 \\
0 & 0 \\
0 & 0\end{array}$ & $\begin{array}{ll}N & \infty \\
& \infty \\
0 & 0 \\
0\end{array}$ & 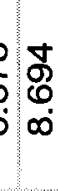 & $\stackrel{8}{\Omega}$ & $\begin{array}{l}0 \\
0 \\
0 \\
\infty\end{array}$ & 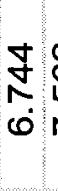 & 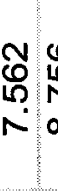 & & $\begin{array}{l}\mathscr{Q} \\
\infty \\
\infty \\
\dot{n}\end{array}$ & & & 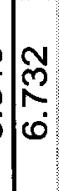 & & $\begin{array}{l}\dot{g} \\
0 \\
\infty\end{array}$ & & $\frac{8}{8}$ & $\begin{array}{l}0 \\
0 \\
\infty\end{array}$ & $\begin{array}{l}\stackrel{n}{m} \\
\stackrel{\leftrightarrow}{-} \\
\sigma\end{array}$ & $\frac{N}{6}$ & $\begin{array}{c}5 \\
5 \\
\infty\end{array}$ & : \\
\hline & 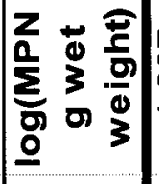 & 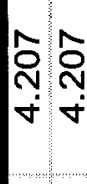 & & $\begin{array}{l}8 \\
0 \\
8\end{array}$ & 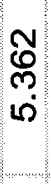 & $\left.\begin{array}{c}\infty \\
N \\
N \\
m\end{array}\right]$ & $\begin{array}{l}3 \\
\infty \\
\infty \\
\dot{8}\end{array}$ & & 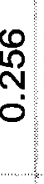 & P & 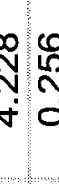 & $\mid$\begin{tabular}{l}
$\hat{N}$ \\
\multirow{\sim}{*}{}
\end{tabular} & & 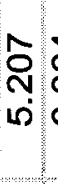 & & 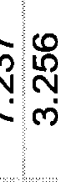 & $\begin{array}{l}\infty \\
6 \\
0\end{array}$ & $\overbrace{0}^{\circ}$ & & 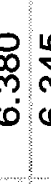 & लि \\
\hline & $\frac{0}{z} \frac{1}{2}$ & $\begin{array}{ll}0 & 0 \\
0 & 0 \\
+ & + \\
\omega & 4 \\
0 & 0 \\
0 & 0 \\
10 & N\end{array}$ & $\left\{\begin{array}{l}\infty \\
0 \\
+ \\
\omega \\
\wp \\
0\end{array}\right.$ & $\begin{array}{l}5 \\
+ \\
+ \\
\infty \\
0 \\
\vdots \\
r \\
r\end{array}$ & $\begin{array}{c}\infty \\
0 \\
+ \\
\sim \\
0 \\
\vdots \\
-\end{array}$ & \begin{tabular}{l}
8 \\
+ \\
4 \\
\multirow{4}{0}{} \\
10
\end{tabular} & 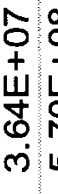 & & 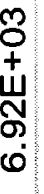 & $\frac{1}{n}$ & 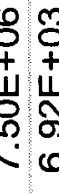 & 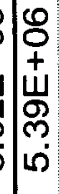 & $\begin{array}{l}8 \\
0 \\
+ \\
山 \\
0 \\
1 \\
\Gamma\end{array}$ & $\begin{array}{l}\infty \\
0 \\
+ \\
w \\
0 \\
0 \\
\dot{\tau}\end{array}$ & 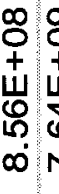 & 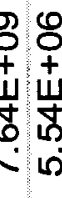 & 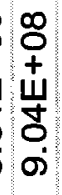 & $\begin{array}{l}8 \\
+ \\
w \\
N \\
\text { N }\end{array}$ & $\begin{array}{l}8 \\
+ \\
+ \\
\\
\\
0\end{array}$ & 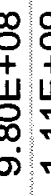 & 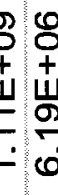 \\
\hline & 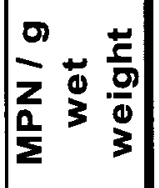 & 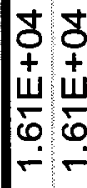 & 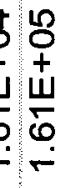 & $\begin{array}{l}8 \\
+ \\
+ \\
\dot{~} \\
\dot{q} \\
\dot{q}\end{array}$ & 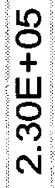 & 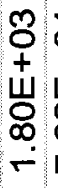 & 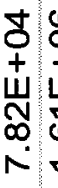 & $\begin{array}{l}8 \\
+ \\
+ \\
\\
0 \\
\vdots \\
\end{array}$ & $\begin{array}{l}8 \\
\\
+ \\
\\
\infty \\
\infty \\
- \\
\end{array}$ & $\therefore$ & 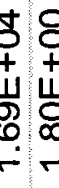 & 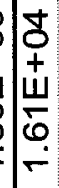 & $\begin{array}{l}\stackrel{+}{0} \\
+ \\
\stackrel{+}{\sigma} \\
\dot{0} \\
-\end{array}$ & $\begin{array}{l}18 \\
0 \\
+ \\
w \\
\sigma \\
0 \\
\sigma\end{array}$ & 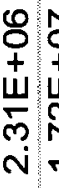 & 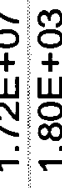 & 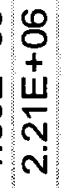 & 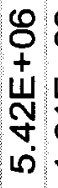 & 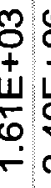 & 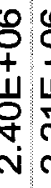 & 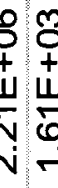 \\
\hline & $\begin{array}{l}\bar{\pi} \\
\stackrel{0}{0} \\
\end{array}$ & 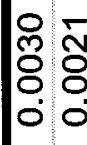 & $\begin{array}{l}8 \\
8 \\
0\end{array}$ & : & 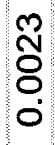 & $\begin{array}{l}8 \\
8 \\
8 \\
0\end{array}$ & & & 8 & 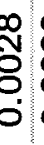 & 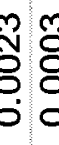 & 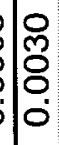 & ๙্. & $\begin{array}{l}m \\
8 \\
8 \\
0\end{array}$ & 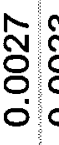 & $\begin{array}{l}2 \\
0 \\
0 \\
0 \\
0\end{array}$ & $\begin{array}{l}4 \\
0 \\
0 \\
0\end{array}$ & $\begin{array}{l}0 \\
8 \\
8 \\
0\end{array}$ & & $\begin{array}{l}\dot{+} \\
8 \\
8 \\
0 \\
\dot{0}\end{array}$ & $\begin{array}{l}3 \\
8 \\
0\end{array}$ \\
\hline $\begin{array}{l}0 \\
\frac{5}{0} \\
8 \\
8\end{array}$ & 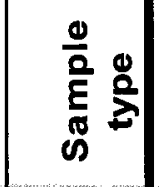 & 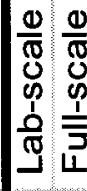 & 告 & $\begin{array}{l}\frac{0}{10} \\
0 \\
0 \\
0 \\
0 \\
0\end{array}$ & 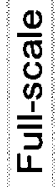 & $\begin{array}{l}\infty \\
\infty \\
0\end{array}$ & $\begin{array}{l}\frac{0}{D} \\
0 \\
1 \\
0 \\
0 \\
\end{array}$ & 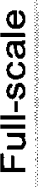 & $\begin{array}{l}\infty \\
\text { a } \\
\alpha\end{array}$ & 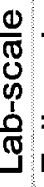 & 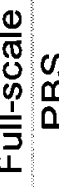 & 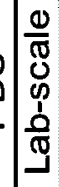 & $\begin{array}{l}\frac{0}{8} \\
\frac{p}{0} \\
\frac{1}{3} \\
4\end{array}$ & o & 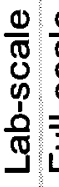 & $\begin{array}{l}\frac{0}{8} \\
0 \\
0 \\
\frac{1}{3} \\
1\end{array}$ & $\begin{array}{l}\frac{0}{0} \\
0 \\
0 \\
\vdots \\
0 \\
\end{array}$ & $\begin{array}{l}\frac{0}{8} \\
0 \\
0 \\
\frac{1}{3} \\
4\end{array}$ & $\begin{array}{l}\infty \\
\mathbf{m} \\
0\end{array}$ & 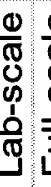 & 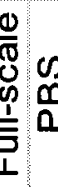 \\
\hline $\begin{array}{l}\overline{8} \\
\mathbf{u} \\
4\end{array}$ & $\overrightarrow{0}$ & 00 & 20 & if & $r$ & $\sigma$ & $\mathrm{No}$ & $N$ & $N$ & 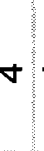 & $\forall \nabla$ & 0 & 0 & 0 & - & $r r$ & $N$ & $N$ & $N$ & 寸 & $\forall \forall$ \\
\hline 竞 & 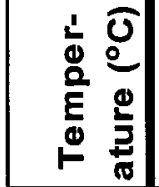 & $\nabla \nabla$ & & $\nabla$ & $\nabla$ & $\nabla$ & $\theta$ & & $\theta$ & $\nabla$ & $\nabla \gamma$ & $\pi$ & $\lambda$ & 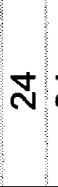 & 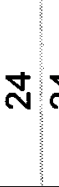 & 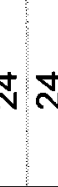 & $N$ & n & $\mathbf{N}$ & $\stackrel{d}{\sim}$ & $\checkmark$ \\
\hline
\end{tabular}




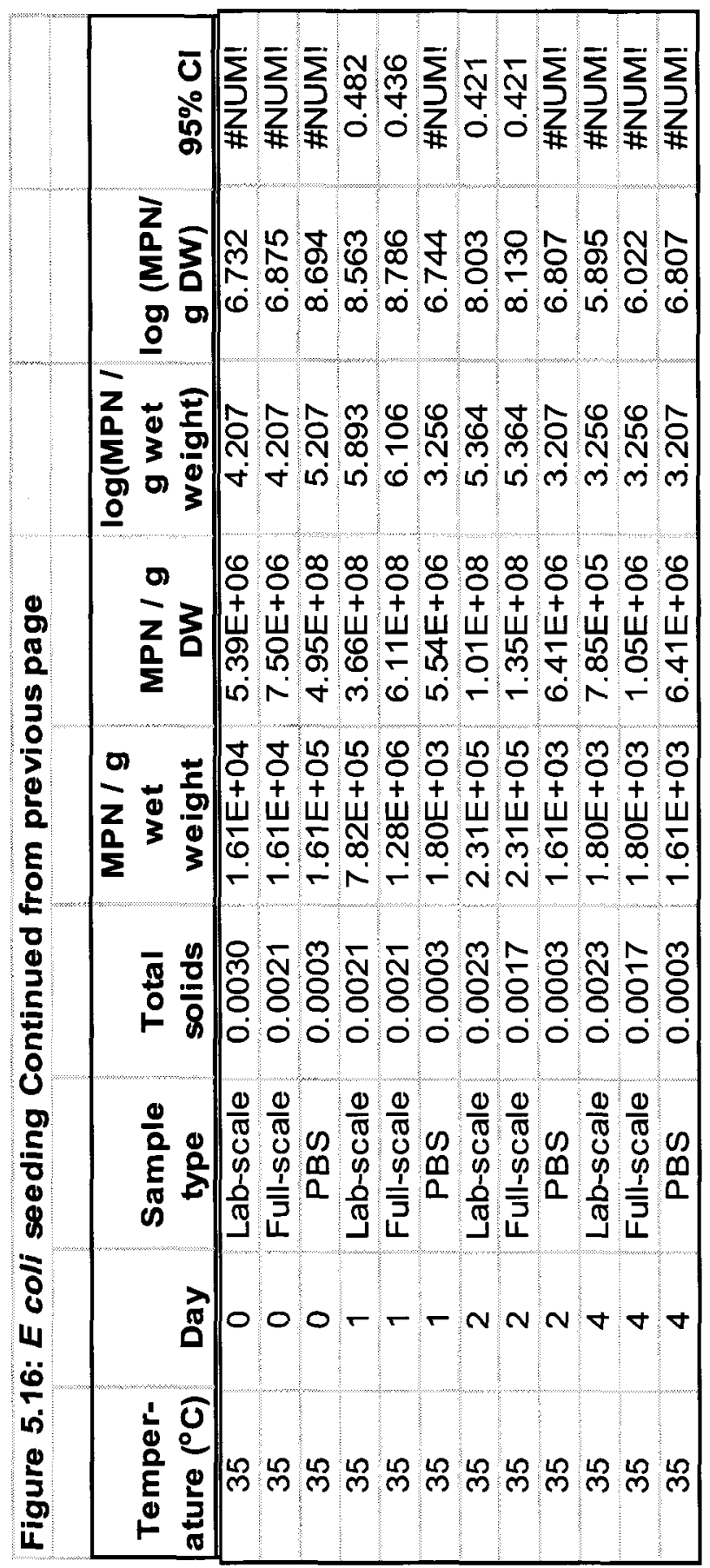




\begin{tabular}{|c|c|c|c|c|c|c|c|c|}
\hline \multicolumn{4}{|c|}{ Figure 5.17-5.24: Luminase Data } & & & & & \\
\hline Sample type & Day & $\begin{array}{l}\text { Temper- } \\
\text { ature }\left({ }^{\circ} \mathrm{C}\right)\end{array}$ & $\begin{array}{c}\text { extraATP } \\
(n g / m L)\end{array}$ & $\begin{array}{c}\text { intraATP } \\
\text { (ng/mL) }\end{array}$ & $\begin{array}{l}\text { BSI } \\
(\%)\end{array}$ & $\begin{array}{l}\text { TS } \\
(\%)\end{array}$ & $\begin{array}{c}\text { intraATP } \\
/ \text { gDW }\end{array}$ & $\begin{array}{c}\text { extraATP } \\
\text { / gDW }\end{array}$ \\
\hline Centrate lab & Pre & NA & 8.35 & 32.63 & 20.37 & 0.40 & 81.59 & 20.86 \\
\hline Centrate full & Pre & NA & 28.98 & 48.06 & 37.62 & 0.42 & 114.44 & 69.00 \\
\hline Digested & Pre & NA & 132.83 & 457.09 & 22.52 & 3.00 & 152.36 & 44.28 \\
\hline Cake & Pre & NA & 164.20 & 939.87 & 14.87 & 30.00 & 31.33 & 5.47 \\
\hline Digester in & 0 & NA & 2646.67 & 3952.83 & 40.10 & 5.32 & 743.71 & 497.96 \\
\hline Digested & 0 & NA & 490.54 & 532.75 & 47.94 & 2.51 & 212.13 & 195.32 \\
\hline Post Polymer & 0 & NA & 95.92 & 288.10 & 24.98 & 2.01 & 143.27 & 47.70 \\
\hline Cake & 0 & NA & 236.31 & 1351.03 & 14.89 & 29.57 & 45.69 & 7.99 \\
\hline Centrate Lab & 0 & NA & 164.51 & 147.01 & 52.81 & 0.32 & 460.39 & 515.22 \\
\hline Centrate Full & 0 & NA & 110.13 & 65.96 & 62.54 & 0.22 & 297.02 & 495.97 \\
\hline Cake & 2 & 4 & 143.40 & 229.40 & 38.47 & 35.02 & 6.55 & 4.10 \\
\hline Cake & 2 & 24 & 178.94 & 312.13 & 36.44 & 34.25 & 9.11 & 5.22 \\
\hline Cake & 2 & 35 & 96.24 & 496.06 & 16.25 & 32.84 & 15.10 & 2.93 \\
\hline Centrate Lab & 2 & 4 & 38.09 & 45.02 & 45.83 & 0.28 & 159.72 & 135.15 \\
\hline Centrate Lab & 2 & 24 & 68.48 & 70.07 & 49.42 & 0.28 & 247.05 & 241.43 \\
\hline Centrate Lab & 2 & 35 & 70.54 & 71.46 & 49.68 & 0.31 & 232.81 & 229.82 \\
\hline Centrate Full & 2 & 4 & 33.56 & 38.41 & 46.63 & 0.23 & 165.00 & 144.13 \\
\hline Centrate Full & 2 & 24 & 79.31 & 78.78 & 50.17 & 0.22 & 352.03 & 354.38 \\
\hline Centrate Full & 2 & 35 & 61.84 & 50.22 & 55.18 & 0.23 & 217.59 & 267.91 \\
\hline Cake & 4 & 4 & 42.33 & 180.96 & 18.96 & 34.06 & 5.31 & 1.24 \\
\hline Cake & 4 & 24 & 253.06 & 841.64 & 23.12 & 33.08 & 25.44 & 7.65 \\
\hline Cake & 4 & 35 & 89.00 & 220.62 & 28.74 & 31.66 & 6.97 & 2.81 \\
\hline Centrate Lab & 4 & 4 & 25.92 & 32.86 & 44.10 & 0.33 & 100.30 & 79.13 \\
\hline Centrate Lab & 4 & 24 & 70.29 & 66.68 & 51.32 & 0.32 & 208.90 & 220.22 \\
\hline Centrate Lab & 4 & 35 & 65.06 & 85.23 & 43.29 & 0.31 & 271.22 & 207.03 \\
\hline Centrate Full & 4 & 4 & 27.66 & 38.91 & 41.55 & 0.25 & 156.17 & 111.02 \\
\hline Centrate Full & 4 & 24 & 42.98 & 87.23 & 33.01 & 0.24 & 361.74 & 178.22 \\
\hline Centrate Full & 4 & 35 & 25.48 & 51.72 & 33.01 & 0.24 & 214.50 & 105.68 \\
\hline
\end{tabular}


Figure 5.17-5.24: Luminase Data Continued from previous page

\begin{tabular}{|c|c|c|c|c|c|c|c|c|}
\hline Sample type & Day & $\begin{array}{l}\text { Temper- } \\
\text { ature }\left({ }^{\circ} \mathrm{C}\right)\end{array}$ & $\begin{array}{c}\text { extraATP } \\
\text { (ng/mL) }\end{array}$ & $\begin{array}{c}\text { Intra ATP } \\
\text { (ng/mL) }\end{array}$ & $\begin{array}{l}\text { BSI } \\
(\%)\end{array}$ & $\begin{array}{l}\text { TS } \\
(\%)\end{array}$ & $\begin{array}{c}\text { intraATP } \\
\text { / gDW }\end{array}$ & $\begin{array}{c}\text { extraATP } \\
\text { / gDW }\end{array}$ \\
\hline Cake & 8 & 4 & 62.77 & 266.46 & 19.07 & 31.90 & 8.35 & 1.97 \\
\hline Cake & 8 & 24 & 132.50 & 324.95 & 28.96 & 31.08 & 10.45 & 4.26 \\
\hline Cake & 8 & 35 & 20.43 & 136.54 & 13.01 & 29.08 & 4.70 & 0.70 \\
\hline Centrate Lab & 8 & 4 & 26.80 & 52.52 & 33.78 & 0.30 & 174.20 & 88.88 \\
\hline Centrate Lab & 8 & 24 & 45.11 & 58.91 & 43.37 & 0.30 & 197.69 & 151.40 \\
\hline Centrate Lab & 8 & 35 & 21.79 & 38.27 & 36.28 & 0.23 & 168.79 & 96.11 \\
\hline Centrate Full & 8 & 4 & 26.75 & 39.35 & 40.47 & 0.23 & 167.60 & 113.94 \\
\hline Centrate Full & 8 & 24 & 38.99 & 40.51 & 49.05 & 0.19 & 210.90 & 203.03 \\
\hline Centrate Full & 8 & 35 & 50.82 & 89.91 & 36.11 & 0.20 & 455.54 & 257.45 \\
\hline Cake & 15 & 4 & 107.29 & 319.41 & 25.14 & 31.67 & 10.09 & 3.39 \\
\hline Cake & 15 & 24 & 77.76 & 315.15 & 19.79 & 31.07 & 10.14 & 2.50 \\
\hline Cake & 15 & 35 & 14.91 & 98.13 & 13.19 & 29.24 & 3.36 & 0.51 \\
\hline Centrate Lab & 15 & 4 & 22.14 & 16.31 & 57.58 & 0.31 & 52.96 & 71.90 \\
\hline Centrate Lab & 15 & 24 & 52.63 & 88.11 & 37.39 & 0.27 & 326.63 & 195.10 \\
\hline Centrate Lab & 15 & 35 & 18.80 & 42.38 & 30.73 & 0.27 & 159.78 & 70.87 \\
\hline Centrate Full & 15 & 4 & 18.20 & 35.79 & 33.72 & 0.22 & 160.72 & 81.75 \\
\hline Centrate Full & 15 & 24 & 38.35 & 27.55 & 58.19 & 0.22 & 124.48 & 173.25 \\
\hline Centrate Full & 15 & 35 & 15.76 & 33.62 & 31.92 & 0.19 & 179.81 & 84.31 \\
\hline Cake & 29 & 24 & 242.99 & 832.47 & 22.59 & 31.39 & 26.52 & 7.74 \\
\hline Cake & 29 & 35 & 39.13 & 240.71 & 13.98 & 28.43 & 8.47 & 1.38 \\
\hline Centrate Lab & 29 & 4 & 13.89 & 29.18 & 32.26 & 0.28 & 103.62 & 49.34 \\
\hline Centrate Lab & 29 & 24 & 17.46 & 55.15 & 24.05 & 0.28 & 194.99 & 61.75 \\
\hline Centrate Lab & 29 & 35 & 14.27 & 42.24 & 25.25 & 0.25 & 169.64 & 57.30 \\
\hline Centrate Full & 29 & 4 & 158.04 & 43.63 & 78.37 & 0.20 & 215.87 & 782.05 \\
\hline Centrate Full & 29 & 24 & 14.73 & 40.39 & 26.72 & 0.20 & 198.24 & 72.28 \\
\hline Centrate Full & 29 & 35 & 16.03 & 37.38 & 30.02 & 0.20 & 191.35 & 82.07 \\
\hline
\end{tabular}


Figure 5.22 - 5.24: MPN data, cake and centrate samples

\begin{tabular}{|c|c|c|c|c|c|c|c|c|}
\hline Sample type & Day & $\begin{array}{l}\text { Temper- } \\
\text { ature }\left({ }^{\circ} \mathrm{C}\right)\end{array}$ & $\begin{array}{l}\text { TS } \\
(\%)\end{array}$ & $95 \% \mathrm{Cl}$ & $\begin{array}{l}\text { MPN / g } \\
\text { wet } \\
\text { weight }\end{array}$ & $\begin{array}{l}\text { MPN / g } \\
\text { DW }\end{array}$ & $\begin{array}{l}\log M P N / \\
g \text { wet } \\
\text { weight }\end{array}$ & $\begin{array}{l}\log M P N \\
\text { gDW }\end{array}$ \\
\hline Digester in & 0 & NA & 5.32 & & 1.97E+06 & $3.70 \mathrm{E}+07$ & 6.29 & 7.57 \\
\hline Digested & 0 & NA & 2.51 & & $1.63 E+03$ & 6.47E+04 & 3.21 & 4.81 \\
\hline Post Polymer & 0 & NA & 2.01 & & $1.25 E+03$ & $6.24 \mathrm{E}+04$ & 3.10 & 4.80 \\
\hline Cake & 0 & NA & 29.57 & 0.438 & $1.30 \mathrm{E}+04$ & $4.39 E+04$ & 4.11 & 4.64 \\
\hline Centrate Lab & 0 & NA & 0.32 & \#NUM! & $1.80 E+01$ & $5.65 E+03$ & 1.26 & 3.75 \\
\hline Centrate Full & 0 & NA & 0.22 & 0.421 & $2.31 E+02$ & $1.04 \mathrm{E}+05$ & 2.36 & 5.02 \\
\hline Cake & 2 & 4 & 35.02 & 0.446 & $3.29 E+03$ & $9.40 \mathrm{E}+03$ & 3.52 & 3.97 \\
\hline Cake & 2 & 24 & 34.25 & 0.429 & $2.40 \mathrm{E}+06$ & $7.00 \mathrm{E}+06$ & 6.38 & 6.85 \\
\hline Cake & 2 & 35 & 32.84 & 0.421 & $2.31 E+05$ & $7.04 \mathrm{E}+05$ & 5.36 & 5.85 \\
\hline Centrate Lab & 2 & 4 & 0.28 & 0.484 & $7.92 E+00$ & $2.81 E+03$ & 0.90 & 3.45 \\
\hline Centrate Lab & 2 & 24 & 0.28 & 0.453 & $1.09 \mathrm{E}+01$ & $3.83 E+03$ & 1.04 & 3.58 \\
\hline Centrate Lab & 2 & 35 & 0.31 & 0.429 & $2.40 \mathrm{E}+01$ & $7.81 E+03$ & 1.38 & 3.89 \\
\hline Centrate Full & 2 & 4 & 0.23 & 0.429 & $2.40 \mathrm{E}+02$ & $1.03 E+05$ & 2.38 & 5.01 \\
\hline Centrate Full & 2 & 24 & 0.22 & 0.446 & $3.29 E+01$ & 1.47E+04 & 1.52 & 4.17 \\
\hline Centrate Full & 2 & 35 & 0.23 & 0.489 & $4.93 E+01$ & $2.14 \mathrm{E}+04$ & 1.69 & 4.33 \\
\hline Cake & 4 & 4 & 34.06 & 0.489 & $4.93 E+04$ & $1.45 E+05$ & 4.69 & 5.16 \\
\hline Cake & 4 & 24 & 33.08 & 0.505 & $9.18 \mathrm{E}+07$ & 2.77E+08 & 7.96 & 8.44 \\
\hline Cake & 4 & 35 & 31.66 & 0.421 & $2.31 E+06$ & $7.30 \mathrm{E}+06$ & 6.36 & 6.86 \\
\hline Centrate Lab & 4 & 4 & 0.33 & 0.493 & $7.00 \mathrm{E}+00$ & $2.14 E+03$ & 0.84 & 3.33 \\
\hline Centrate Lab & 4 & 24 & 0.32 & 0.446 & $3.29 E+00$ & $1.03 E+03$ & 0.52 & 3.01 \\
\hline Centrate Lab & 4 & 35 & 0.31 & 0.426 & $2.21 E+01$ & 7.04E+03 & 1.34 & 3.85 \\
\hline Centrate Full & 4 & 4 & 0.25 & 0.429 & $2.40 E+02$ & $9.62 \mathrm{E}+04$ & 2.38 & 4.98 \\
\hline Centrate Full & 4 & 24 & 0.24 & 0.473 & $1.61 E+02$ & $6.67 E+04$ & 2.21 & 4.82 \\
\hline Centrate Full & 4 & 35 & 0.24 & 0.505 & $9.18 \mathrm{E}+01$ & $3.81 E+04$ & 1.96 & 4.581 \\
\hline
\end{tabular}


Figure 5.22 - 5.24: MPN data, cake and centrate samples Continued from previous page

\begin{tabular}{|c|c|c|c|c|c|c|c|c|}
\hline Sample type & Day & $\begin{array}{l}\text { Temper- } \\
\text { ature }\left({ }^{\circ} \mathrm{C}\right)\end{array}$ & $\begin{array}{l}\text { TS } \\
(\%)\end{array}$ & $95 \% \mathrm{Cl}$ & $\begin{array}{l}\text { MPN / g } \\
\text { wet } \\
\text { weight }\end{array}$ & $\begin{array}{l}\text { MPN / g } \\
\text { DW }\end{array}$ & $\begin{array}{l}\text { logMPN / } \\
\text { g wet } \\
\text { weight }\end{array}$ & $\begin{array}{c}\log M P N / \\
\text { gDW }\end{array}$ \\
\hline Cake & 8 & 4 & 31.90 & 0.489 & $4.93 E+03$ & $1.55 \mathrm{E}+04$ & 3.69 & 4.19 \\
\hline Cake & 8 & 24 & 31.08 & 0.463 & $3.48 E+06$ & $1.12 E+07$ & 6.54 & 7.05 \\
\hline Cake & 8 & 35 & 29.08 & \#NUM! & $1.80 E+04$ & $6.20 E+04$ & 4.26 & 4.79 \\
\hline Centrate Lab & 8 & 4 & 0.30 & 0.446 & $3.29 E+00$ & $1.09 \mathrm{E}+03$ & 0.52 & 3.04 \\
\hline Centrate Lab & 8 & 24 & 0.30 & 0.489 & $4.93 E+00$ & $1.66 \mathrm{E}+03$ & 0.69 & 3.22 \\
\hline Centrate Lab & 8 & 35 & 0.23 & \#NUM! & $1.80 E-01$ & $7.95 \mathrm{E}+01$ & 0.00 & 1.90 \\
\hline Centrate Full & 8 & 4 & 0.23 & 0.426 & $2.21 E+02$ & $9.42 E+04$ & 2.34 & 4.97 \\
\hline Centrate Full & 8 & 24 & 0.19 & 0.473 & $1.61 E+02$ & $8.38 \mathrm{E}+04$ & 2.21 & 4.92 \\
\hline Centrate Full & 8 & 35 & 0.20 & & $2.90 E-01$ & $1.47 E+02$ & 0.00 & 2.17 \\
\hline Cake & 15 & 4 & 31.67 & 0.421 & $2.31 \mathrm{E}+03$ & $7.30 \mathrm{E}+03$ & 3.36 & 3.86 \\
\hline Cake & 15 & 24 & 31.07 & 0.600 & 4.47E+04 & $1.44 E+05$ & 4.65 & 5.158 \\
\hline Cake & 15 & 35 & 29.24 & \#NUM! & $1.80 E+02$ & $6.17 \mathrm{E}+02$ & 2.26 & 2.79 \\
\hline Centrate Lab & 15 & 4 & 0.31 & 0.446 & $3.29 E+00$ & $1.07 E+03$ & 0.52 & 3.03 \\
\hline Centrate Lab & 15 & 24 & 0.27 & \#NUM! & $1.99 E-01$ & $7.37 E+01$ & 0.00 & 1.87 \\
\hline Centrate Lab & 15 & 35 & 0.27 & \#NUM! & $1.80 E-01$ & $6.80 E+01$ & 0.00 & 1.83 \\
\hline Centrate Full & 15 & 4 & 0.22 & 0.446 & $3.29 E+01$ & $1.48 E+04$ & 1.52 & 4.17 \\
\hline Centrate Full & 15 & 24 & 0.22 & 0.484 & $7.92 E+00$ & $3.58 E+03$ & 0.90 & 3.55 \\
\hline Centrate Full & 15 & 35 & 0.19 & \#NUM! & $1.80 E-01$ & $9.64 E+01$ & 0.00 & 1.98 \\
\hline
\end{tabular}


Figure 5.22 - 5.24: MPN data, cake and centrate samples Continued from previous page

\begin{tabular}{|c|c|c|c|c|c|c|c|c|}
\hline Sample type & Day & $\begin{array}{l}\text { Temper- } \\
\text { ature }\left({ }^{\circ} \mathrm{C}\right)\end{array}$ & TS $(\%)$ & $95 \% \mathrm{Cl}$ & $\begin{array}{l}\text { MPN / g } \\
\text { wet } \\
\text { weight }\end{array}$ & $\begin{array}{l}\text { MPN / g } \\
\text { DW }\end{array}$ & $\begin{array}{l}\text { logMPN / } \\
\text { g wet } \\
\text { weight }\end{array}$ & $\begin{array}{c}\log M P N / \\
\text { gDW }\end{array}$ \\
\hline Cake & 22 & 4 & 31.38 & 0.493 & $7.58 E+02$ & $2.42 E+03$ & 2.88 & 3.383 \\
\hline Cake & 22 & 24 & 30.66 & 0.438 & $1.30 E+04$ & $4.24 E+04$ & 4.11 & 4.627 \\
\hline Cake & 22 & 35 & 29.21 & \#NUM! & $1.80 E+00$ & $6.17 E+00$ & 0.26 & 0.791 \\
\hline Centrate Lab & 22 & 4 & 0.29 & 0.446 & $3.29 E+00$ & $1.15 E+03$ & 0.52 & 3.062 \\
\hline Centrate Lab & 22 & 24 & 0.21 & \#NUM! & $1.37 \mathrm{E}-01$ & $6.60 \mathrm{E}+01$ & 0.00 & 1.820 \\
\hline Centrate Lab & 22 & 35 & 0.27 & \#NUM! & $1.80 \mathrm{E}-01$ & $6.67 E+01$ & 0.00 & 1.824 \\
\hline Centrate Full & 22 & 4 & 0.21 & 0.463 & $3.48 E+01$ & $1.63 E+04$ & 1.54 & 4.212 \\
\hline Centrate Full & 22 & 24 & 0.22 & 0.436 & $1.28 \mathrm{E}+00$ & $5.91 \mathrm{E}+02$ & 0.11 & 2.772 \\
\hline Centrate Full & 22 & 35 & 0.20 & \#NUM! & 1.80E-01 & 9.13E+01 & 0.00 & 1.960 \\
\hline Cake & 29 & 4 & 36.75 & 0.489 & $4.93 E+02$ & $1.34 \mathrm{E}+03$ & 2.69 & 3.128 \\
\hline Cake & 29 & 24 & 31.39 & 0.421 & $2.31 E+03$ & $7.36 E+03$ & 3.36 & 3.867 \\
\hline Cake & 29 & 35 & 28.43 & \#NUM! & $1.80 E+00$ & $6.34 \mathrm{E}+00$ & 0.26 & 0.802 \\
\hline Centrate Lab & 29 & 4 & 0.28 & 0.421 & $2.31 E+00$ & $8.21 E+02$ & 0.36 & 2.914 \\
\hline Centrate Lab & 29 & 24 & 0.28 & \#NUM! & $1.80 \mathrm{E}-01$ & $6.38 \mathrm{E}+01$ & 0.00 & 1.804 \\
\hline Centrate Lab & 29 & 35 & 0.25 & \#NUM! & $1.80 \mathrm{E}-01$ & $7.24 E+01$ & 0.00 & 1.860 \\
\hline Centrate Full & 29 & 4 & 0.20 & 0.429 & $2.40 E+01$ & $1.19 \mathrm{E}+04$ & 1.38 & 4.074 \\
\hline Centrate Full & 29 & 24 & 0.20 & \#NUM! & $1.80 \mathrm{E}-01$ & $8.85 E+01$ & 0.00 & 1.947 \\
\hline Centrate Full & 29 & 35 & 0.20 & \#NUM! & $1.80 \mathrm{E}-01$ & $9.23 E+01$ & 0.00 & 1.965 \\
\hline
\end{tabular}

